\title{
The processing of German Sign Language sentences
}

\author{
Three event-related potential studies on phonological, \\ morpho-syntactic, and semantic aspects
}

\begin{abstract}
Dissertation
zur Erlangung des philosophischen Doktorgrades

an der Philosophischen Fakultät der Georg-August-Universität
\end{abstract}

vorgelegt von

Jana Hosemann

aus Berlin

Göttingen, 2015 
Erstgutachter: Prof. Dr. Markus Steinbach

Zweitgutachter: Prof. Dr. Matthias Schlesewsky

Drittgutachter: Dr. Nivedita Mani

Datum der mündlichen Prüfung (Disputation): 10. April 2015 
To the German Deaf community ... and all signers 


\section{Acknowledgements}

Mountaineering is not an individual sport. Although, when facing the distance between the next step and the desired mountain peak the alpinist is very much on her own. But, even if dealing with stormy emotions, thin air or dizziness in high altitude is left to the alpinist herself, reaching the summit of the mountain is an achievement of an entire crew. Hereby, I wish to express my gratitude for the wonderful support that I have received during this journey and which has led to a truly unique experience.

The basic idea of starting this mountaineering project was in fact born a couple of years ago in a climbing gym. I realized at that point that many coincidences had come together, which laid the foundation for the commencement of this journey: I spent half a year in a mountaineering training camp in Frankfurt. There, I had the chance to meet Annika Herrmann (alias Annike), who was the first person who showed me a way this journey could go. Her wonderful enthusiastic joy of mountain climbing opened up new and exciting ways of thinking, so that I saw the possibility of following her directions. Through her, I had the privilege of meeting Markus Steinbach and Matthias Schlesewksy, who became my superb coaches. Not only did they coach me along the way during this project, they also showed me a world beyond. In many extremely productive meetings with Matthias, I was inspired and captured by his enthusiasm and energy. In many frequent expeditions to Mainz, I learned all the necessary skills needed for the hardware of this journey. Matthias, Ina Bornkessel-Schlesewsky, Franziska Kretzschmar, Markus Phillip, Petra Schumacher and the team in Mainz were excellent teachers who provided the necessary equipment and instructed me in the handling of the technology. It is here where I learned standard (and nonstandard) approaches to the techniques, and how to find creative solutions. I benefited greatly from my time in Mainz, because they showed me, what could be achieved by an entire team that a single person could never attain. 
This very same team spirit also grew in Göttingen. Markus and Annike built the fundaments of this great 'mountain climbing cadre'. Thanks to their engagement and dedication, the 'SLT Gö' became a training camp as well as a place of security. Markus' superb supervision in many ways and his encouragement made it possible for me to continue the journey despite unforeseen challenges. While working on approaching the base camp and actually beginning the climb, I learned a lot from meetings with people on an everyday basis. Nivedita Mani and Nicole Altvater-Mackensen from the neighbor squad supplied a lot of help in order to make an important progress. Thanks to them, we came closer to setting up the base camp. In addition, the practice sessions with the 'AK' kept the ball rolling. So, thanks to Annika Hübl (alias Annikü), Anna Boell, Nina Pendzich, Elisabeth Volk, Sina Jahnke, Julia Krebs, und Sukie Brinkmann for training with me. Consequently, reaching this mountain summit is based much on the support and encouragement of Markus, Annike, Annikü, Roland Metz, and Nina. I am delighted to see that in the last few years, the 'SLT Gö' has grown in so many ways that I can't imagine how mountaineering will be without them. However, I am extremely pleased we have managed to recruit new members to the team: Liona Paulus, Derya Nuhbalaoglu, and Anne Wienholz. I hope I can be as supportive to their massif projects as the others have been in supporting me.

Of course, even reaching the base camp would not have been possible without the knowledge of experts. So, I am more than happy, that on my way I met many of them. They laid the groundwork, so I could actually start approaching the summit. Daniela Happ, Roland and Liona taught me a lot about the matter I would deal with. In our meetings and conversations, I could discuss and practice with them while also having a lot of fun. Annette Flemnitz, Konstantin Grin, the specialists around Göttingen, and especially the experts participating in this project contributed enormously. Without their knowledge and their willingness to share it with me, I wouldn't have reached the base camp. I am amazed how much time and effort they invested in supporting this project. 
With all the hard skills I had learned and all the soft skills I had acquired during training, with all the support of so many people who I was fortunate to meet along the way, at one day I reached base camp and faced this intimidating wall in front of me. Now was the time to actually apply all these skills. And although the alpinist has to take each step by herself and find her own pace, there are other people directly and indirectly accompanying and guiding her. Dagmar and Wilfried planted the seed for enjoying mountaineering and encouraged me to be adventurous in the first place. In the last months, Wilfried and Gudrun fuelled me on my hike in so many ways. They assisted me via 'walkie talkie', guided me through the final steps, and often motivated me to make the next move. On stormy or cloudy days, Anna, Sharmishtha, and Chris often reminded me that there is sun above the clouds and that there is a great view waiting for me on top. When the head aches due to a lack of oxygen, it takes courage to take a step back and descend. After a short pause the next altitude difference is often reached with much more ease. And thanks to Josep, I got equipped with a "Quer" package for the last phase. Scrambling on the wall from camp to camp, it was Pierre most of all who accompanied me. Without his support, his patience and his nutrition, it would not have been possible for me to keep up the pace from camp three all the way to the summit. Now, reaching the top, exhaustion and excitement spreads, but most importantly there is gratitude. So, to the entire supporting crew: "Thank you for making this summit possible!" 


\section{Abstract (English)}

Sign languages have often been the subject of imaging studies investigating the underlying neural correlates of sign language processing. To the contrary, much less research has been conducted on the time-course of sign language processing. There are only a small number of event-related potential (ERP) studies that investigate semantic or morpho-syntactic anomalies in signed sentences. Due to specific properties of the manual-visual modality, sign languages differ from spoken languages in two respects: On the one hand, they are produced in a three-dimensional signing space, on the other hand, sign languages can use several (manual and nonmanual) articulators simultaneously. Thus, sign languages have modality-specific characteristics that have an impact on the way they are processed. This thesis presents three ERP studies on different linguistic aspects processed in German Sign Language (DGS) sentences. Chapter 1 investigates the hypothesis of a forward model perspective on prediction. In a semantic expectation mismatch design, deaf native signers saw videos with DGS sentences that ended in semantically expected or unexpected signs. Since sign languages entail relatively long transition phases between one sign and the next, we tested whether a prediction error of the upcoming sign is already detectable prior to the actual sign onset. Unexpected signs engendered an N400 previous to the critical sign onset that was thus elicited by properties of the transition phase. Chapter 2 presents a priming study on cross-modal cross-language co-activation. Deaf bimodal bilingual participants saw DGS sentences that contained primetarget pairs in one of two priming conditions. In overt phonological priming, prime and target signs were phonologically minimal pairs, while in covert orthographic priming, German translations of prime and target were orthographic minimal pairs, but there was no overlap between the signs. Target signs with overt phonological or with covert orthographic overlap engendered a reduced negativity in the electrophysiological signal. Thus, deaf bimodal bilinguals co-activate their second language (written) German unconsciously during processing sentences in their native sign language. 
Chapter 3 presents two ERP studies investigating the morpho-syntactic aspects of agreement in DGS. One study tested DGS sentences with incorrect, i.e. unspecified, agreement verbs, the other study tested DGS sentences with plain verbs that incorrectly inflected for $3^{\text {rd }}$ person agreement. Agreement verbs that ended in an unspecified location engendered two independent ERP effects: a positive deflection on posterior electrodes (220-570 ms relative to trigger nonmanual cues) and an anterior effect on left frontal electrodes (300$600 \mathrm{~ms}$ relative to the sign onset). In contrast, incorrect plain verbs resulted in a broadly distributed positive deflection $(420-730 \mathrm{~ms}$ relative to the mismatch onset). These results contradict previous findings of agreement violation in sign languages and are discussed to reflect a violation of wellformedness or processes of context-updating. The stimulus materials of these studies were consistently presented in continuously signed sentences presented in non-manipulated videos. This methodological innovation enabled a distinctive perspective on the time-course of sign language processing.

\section{Abstract (German)}

Es gibt zahlreiche Studien, die die kognitive Verarbeitung von Gebärdensprachen mit Hilfe von bildgebenden Verfahren, wie bspw. fMRI, PET oder MEG, untersuchen. Interessanterweise gibt es hingegen nur sehr wenige Studien, die den zeitlichen Verlauf der Gebärdensprachverarbeitung mittels Ereignis-korrelierter Potentiale (EKP) erforschen. Folglich ist die Anzahl der EKP-Studien, die sich semantische und morpho-syntaktische Aspekte von Gebärdensprachen angeschaut haben, sehr gering. Ein Grund für diese geringe Anzahl von EKP-Studien über Gebärdensprachen liegt wohlmöglich in zwei modalitäts-spezifische Eigenschaften, die Gebärdensprachen in ihrer manuell-visuellen Modalität auszeichnen: Zum einen werden Gebärdensprachen in einem drei-dimensionalen Gebärdenraum produziert, zum anderen können bei der Produktion von Gebärdensprachen mehrere Arti- 
kulatoren (manuelle und nicht-manuelle) gleichzeitig beteiligt sein. Diese beiden Charakteristika Dreidimensionalität und Simultanität haben einen erheblichen Einfluss auf die kognitive Verarbeitungsweise von Gebärdensprachen. In der vorliegenden Dissertation werden drei individuelle EKPStudien vorgestellt, die phonologische, morpho-syntaktische und semantische Aspekten der Deutschen Gebärdensprache (DGS) innerhalb der Verarbeitung von DGS-Sätze untersucht haben. In Kapitel 1 wird eine Studie zur semantischen Erwartungsverletzung präsentiert. Basierend auf der Annahme eines "Forward-Models", haben wir die zeitliche Dimension von Vorhersagen zum kommenden lexikalischen Material untersucht. Gehörlose, muttersprachliche Signer sahen Videos mit grammatisch korrekten DGS-Sätzen, die eine semantische Erwartung auf das satzfinale Verb erzeugt haben. Diese Erwartung wurde entweder durch das erwartete Verb bestätigt oder durch ein semantisch unerwartetes Verb verletzt. Da in Gebärdensprachen der Übergang von einer Gebärde zur nächsten verhältnismäßig lang ist (im Vergleich zu den Übergängen zwischen gesprochenen Wörtern in einer Lausptache), konnten wir testen, ob die Verletzung einer lexikalischen Vorhersage bereits vor dem Beginn der kritischen Gebärden erkannt wurde. Interessanterweise haben semantisch unerwartete Verben einen N400 Effekt ausgelöst, der bereits von Informationen innerhalb der Übergangsphase, also vor dem eigentlichen Verbanfang, bedingt wurde. In Kapitel 2 präsentieren wir eine phonologische Priming-Studie zu cross-modaler Ko-Aktivierung einer Lautsprache während der Verarbeitung einer Gebärdensprache. Bimodale, bilinguale gehörlose Muttersprachler (d.h. Gehörlose, die eine Gebärdensprache (L1) und eine Lautsprache (L2) auf hohem Niveau beherrschen), haben Videos mit DGS Sätzen gesehen, in denen eine Primeund eine Target-Gebärde enthalten waren. Prime- und Target-Gebärden waren entweder overt phonologische Minimalpaare in DGS oder covert orthographische Minimalpaare in der deutschen Übersetzung. Die Ergebnisse zeigten, dass sowohl Target-Gebärden, die eine gebärdensprachphonologische Ähnlichkeit zu ihrem Prime hatten, als auch solche TargetGebärden, die als Gebärden phonologisch unrelatiert waren, aber deren 
deutschen Übersetzungen orthographisch mit dem Prime überlappten, zu einem geringeren negativen elektrophysiologischen Signal führten, als die gänzlich unrelatierten Kontroll-Gebärden. Daraus lässt sich schlussfolgern, dass gehörlose Muttersprachler unbewußt die sub-lexikalischen Repräsentationen von (geschriebene) deutschen Wörten ko-aktivieren, während sie Sätze in ihrer Muttersprache DGS verarbeiten. In Kapitel 3 präsentieren wir eine EKP-Studie, die die morpho-syntaktischen Aspekte des KongruenzSystems in DGS untersucht hat. In zwei aufeinander aufbauenden Studien haben wir die Verarbeitung von (a) Kongruenzverletzung bei Kongruenzverben und (b) Kongruenzverletzung bei einfachen Verben getestet. Kongruenzverben, die inkorreterweise an einem unspezifizierten ort im Gebärdenraum endeten (und nicht an dem Ort, der mit dem bezugnehmenden Referenten assoziiert war), führten zu zwei unabhängigen EKP-Effekten: Erstens, einer posterioren Positivierung (in dem Zweitfenster 220-570 ms relativ zum Trigger "nichmanuelle Merkmale"); und zweitens, einem frontalen, anterioren Effekt, dessen Polarität ungewiss ist (300-600 ms relativ zum Trigger "Gebärden Onset”). Im Gegensatz zu diesen Effekten haben flektierte einfache Verben, die grundsätzlich nicht für Flektion spezifiziert sind, eine breit verteilte Positivierung ausgelöst (420-730 ms relativ zum Trigger "Mismatch Onset"). Diese Ergebnisse stehen im Gegensatz zu vorher veröffentlichten Ergebnissen zu Kongruenzverletzungen in Gebärensprachverarbeitung und werden in ihrer funktionalen Bedeutung in Bezug auf Wohlgeformtheits-Verletzung und Kontext-Updating diskutiert. Das Besondere dieser Arbeit ist die Präsentation des Stimulusmaterials. Im Gegensatz zu vorher veröffentlichten EKP-Studien mit Gebärdensprachen, haben wir die zu untersuchenden Aspkete in ganzen Sätzen präsentiert, die in kontinuierlichen und unmanipulierten Videos gezeigt wurden. Diese methodische Innovation ermöglichte es, eine distinktive Perspektive auf den zeitlichen Verlauf der kognitiven Verarbeitung von Gebärdensprachen zu bekommen. 


\section{Table of contents}

$\begin{array}{lr}\text { Abstract (English) } & \text { iv } \\ \text { Abstract (German) } & \mathrm{v} \\ \text { List of figures } & \mathrm{x} \\ \text { List of tables } & \mathrm{xi} \\ \text { Introduction } & 1\end{array}$

1 Lexical prediction via forward models: N400 evidence from German Sign Language $\quad 13$

1.1 Introduction 13

1.1.1 Modeling prediction in language processing 14

1.1.2 Predicting input in a sign language: Simultaneity and threedimensionality 16

1.1.3 Previous electrophysiological studies on sign language processing 18

$\begin{array}{ll}\text { 1.1.4 The present study } & 20\end{array}$

1.2 Materials and methods $\quad 24$

$\begin{array}{ll}\text { 1.2.1 Participants } & 24\end{array}$

$\begin{array}{ll}\text { 1.2.2 Materials } & 24\end{array}$

$\begin{array}{ll}1.2 .3 \text { Procedure } & 27\end{array}$

1.2.4 EEG recording 28

1.2.5 EEG data preprocessing and statistical analysis 28

$\begin{array}{lll}1.3 & \text { Results } & 30\end{array}$

$\begin{array}{ll}\text { 1.3.1 Behavioural data } & 30\end{array}$

1.3.2 ERP data 31

$\begin{array}{ll}1.4 \text { Discussion } & 36\end{array}$

$\begin{array}{lll}1.5 & \text { Summary and conclusions } & 40\end{array}$

2 Signs activate their written word translation. An ERP study on cross-modal co-activation in German Sign Language $\quad 41$

2.1 Introduction $\quad 41$

2.1.1 Phonological priming in sign languages $\quad 43$

$\begin{array}{ll}\text { 2.1.2 Cross-language co-activation } & 47\end{array}$

2.1.3 Previous cross-language co-activation studies with bimodal bilinguals 49

2.1.4 The present study 53 
2.2 Materials and methods $\quad 57$

2.2.1 Participants $\quad 57$

2.2.2 Materials 58

2.2.3 Procedure 61

2.2.4 EEG recording 62

2.2.5 EEG data preprocessing and statistical analysis 63

2.3 Results $\quad 66$

2.3.1 Behavioral data 66

2.3.2 ERP data 66

$\begin{array}{ll}2.4 \text { Discussion } & 69\end{array}$

2.5 Summary and conclusions $\quad 84$

3 Agreement or no agreement. ERP correlates of verb agreement violation in German Sign Language 85

3.1 Introduction 85

3.1.1 Modality-independent properties of sign language agreement

3.1.2 Modality-specific properties of sign language agreement $\quad 95$

3.1.3 Previous electrophysiological studies on sign language agreement violation 101

3.1.4 The two present studies 106

3.2 Experiment A: Agreement violation with agreement verbs 109

3.2.1 Exp. A: Materials and methods 109

3.2.2 Exp. A: Results 114

$\begin{array}{ll}3.2 .3 \text { Exp. A: Discussion } & 117\end{array}$

3.3 Experiment B: Agreement violation with plain verbs 126

3.3.1 Exp. B: Materials and methods 126

3.3.2 Exp. B: Results 131

3.3.3 Exp. B: Discussion 134

3.4 General discussion 136

3.5 Summary and conclusions 143

Conclusion 144

$\begin{array}{ll}\text { References } & 150\end{array}$

Appendix A - Stimulus materials $\quad 168$

Appendix B - Supplementary ERP figures and material 177 


\section{List of figures}

Figure 1.1 Schematic illustration of the transition phase between two signs.

Figure 1.2 Trajectory for the change of location between the previous sign RABBIT and the following sign JUMP.

Figure 1.3 Video stills of the sign onset (left) and the sign offset (right) of the nonaction verb SIT

Figure 1.4 Video stills of the sign onset (left) and the sign offset (right) of the action verb JUMP.

Figure 1.5 Schematic illustration of the trigger positions for ERP time-locking within the transition phase, as well as corresponding video stills for each trigger.

Figure 1.6 Grand average ERPs for unexpected (red line) and expected (blue line) action verbs, time-locked to the handshape trigger.

Figure 1.7 Grand average ERPs for unexpected (red line) and expected (blue line) non-action verbs, time-locked to the target handshape trigger . .33

Figure 1.8 Summary of the statistical analyses in successive $50 \mathrm{~ms}$ time windows for the lateral electrodes.

Figure 1.9 Summary of the statistical analyses in successive $50 \mathrm{~ms}$ time windows for the midline electrodes.

Figure 2.1 Video stills of the signs STORE (left) and ANIMAL (right). The distinctive parameter is the movement.

Figure 2.2 Schematic illustration of a signing stream, including transition phases and the time point of trigger "sign onset".

Figure 2.3 Grand average ERPs for (A) target signs in DGS priming condition (primed targets $=$ dark blue, unprimed control targets $=$ dark red) and for (B) target signs in German priming condition (primed targets = bright blue, unprimed targets $=$ bright red).

Figure 2.4 Schematic illustration of co-activation pathways as proposed by Ormel et al. (2012): Deaf Bilingual Interactive Activation model (p. 301)

Figure 2.5 Schematic illustration of co-activation pathways in hearing bimodal bilinguals as proposed by Shook \& Marian (2012)

Figure 2.6 Schemativ illustration of the sub-lexical representations of a sign and a word (for deaf and hearing people)

Figure 2.7 Schematic illustration of cross-modal cross-language co-activation. (a) explanation via a semantic mediation, (b) explanation via a direct associative link, and (c) explanation via mouthing.

Figure 3.1 Pictures of the sign HELP.

Figure 3.2 Pictures of the verbs EXPLAIN (left, orientation change only), CRITIQUE (middle; movement and orientation change), and GIVE (upper right, movement change only). 
Figure 3.3 Pictures of the DGS plain verbs LIKE, BUY, and PLAY (from left to right) that display a lexically specified movement.

Figure 3.4 Original video stills of the final hold of the critical verb INFORM. (a) in its matching form, agreeing with the $3^{\text {rd }}$ person referent ( INFORM $_{3 a}$ ), and (b) in its mismatching form, ending at an unspecified R-locus on the left side of the signer ( ${ }_{1}$ INFORM $\left._{3 \mathrm{~b}}\right)$.

Figure 3.5 Original video stills of the critical plain verb BUY. (a) in its lexical form, as in the sentence $\mathrm{IX}_{1}$ LAPTOP BUY, and (b) with the extended path movement directing at locus $3 \mathrm{a}$, in order to mark agreement with the $3^{\text {rd }}$ person object, as in the sentence IX LAPTOP BUY $_{3 a}$

Figure 3.6 Grand average ERPs for matching (blue line) and mismatching (red line) agreement verbs, time-locked to the nonmanual cue trigger. Negativity plotted upwards.

Figure 3.7 Grand average ERPs for matching (blue line) and mismatching (red line) agreement verbs, time-locked to the sign onset trigger. Negativity plotted upwards.

Figure 3.8 Video stills of the critical plain verb BUY, (a) with the lexical path movement, and (b) with the manipulated path movement ending at locus $3 a$.

Figure 3.9 Grand average ERPs for matching (blue line) and mismatching (red line) plain verbs, time-locked to the trigger sign onset. Negativity plotted upwards.

Figure 3.10 Grand average ERPs for matching (blue line) and mismatching (red linie) plain verbs, time-locked to the eye gaze onset trigger. Negativity plotted upwards.

Figure 3.11 Grand average ERPs for matching (blue line) and mismatching (red linie) plain verbs, time-locked to the mismatch onset trigger. Negativity plotted upwards.

\section{List of tables}

Table 0.1 Overview of existing event-related potential studies on sign languages............. 5

Table 2.1 Overview of studies on cross-language activation with bimodal

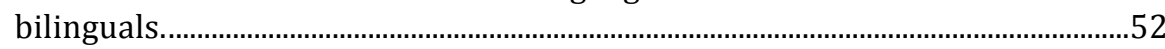

Table 2.2 Overview of participants' metadata. Proficiency was self-evaluated on a 1-10 scale; " $\mathrm{m}$ " = means; standard deviations are shown in parentheses

Table 3.1 List of different kinds of agreement violation allocated to the studies they appeared in and the ERP effects they elicited (R = referent; $\mathrm{L}=$ locus). 


\section{Introduction}

The time-course of processing sign languages has not been investigated in much detail in the past. In fact, there are many more imaging studies (like fMRI, PET, MEG) on sign languages than there are event-related potential (ERP) studies. Although the status of sign languages as natural languages is irrevocably clarified, the modality-specific aspects of its production (manually) and its perception (visually) continuously provide new challenges for theoretical and experimental linguists. The time-course of processing sign language sentences is one of them. The following thesis presents three individual ERP studies on the processing of different linguistic aspects of German Sign Language (DGS): sub-lexical aspects of transition phases between lexical signs (Chapter 1), phonological aspects in cross-modal, crosslanguage co-activation (Chapter 2); and morpho-syntactic and semantic aspects of sign language agreement (Chapter 3). In contrast to previous ERP studies on sign languages, these linguistic aspects were investigated during the processing of whole signed sentences, presented in non-manipulated videos. Thereby, we expanded the methodological conventions and came as close as possible to presenting natural language material. This enabled us to find different results to previous ERP studies and thus to question linguistic canons that originated from spoken languages and were further adapted for sign languages.

The tradition of psycholinguistic research on sign languages is almost as long as its theoretical research, starting 1960 in the United States (Stokoe, 1960; Stokoe, Casterline, \& Cronberg, 1965). With the attempt to investigate the acquisition of American Sign Language (ASL), Klima \& Bellugi (1979) made ground breaking steps in investigating psycholinguistic processes of the sign language used by adults. Ever since, psycholinguistic research on sign languages has experienced an enormous increase. The methodological opportunities, the research questions, and their outcomes are thereby closely connected to the technological development at the time. The first processing 
studies were gating experiments that worked with analogue video cameras, an editing program and a video recorder to present the gating tape (Grosjean, 1981; Clark \& Grosjean, 1982; Emmorey \& Corina, 1990). Later on, video cameras turned digital, computers became a standard technological equipment, and annotation tools were developed. Priming studies and sign spotting studies that recorded participants reaction times and error rates emerged, as presented in detail by Dye (2012).

A second branch of psycholinguistic research on sign languages consists of production studies. With the aim of proving the natural language status of sign languages, these studies followed classical psycholinguistic studies of spoken languages and investigated the tip-of-the-finger phenomenon (Thompson, Emmorey, \& Gollan, 2005) and slips of the hands (Hohenberger, Happ, \& Leuninger, 2002; Leuninger, Hohenberger, Waleschkowski, Menges, \& Happ, 2004). Similar to processing studies, production studies rely on video cameras and annotation tools (see Hohenberger \& Leuninger, 2012). Since production experiments are not relevant for the purpose of the current investigations, we will not go into further detail about them.

Strongly connected to psycholinguistic questions are the questions on how and where in the brain sign languages are processed, the objectives of neurolinguistics (Emmorey, 2002). Poizner, Klima, \& Bellugi (1987) were the first to report investigations of deaf people with aphasia. These presented the first evidence of a left-hemispheric dominance of neural systems involved in the mediation of sign language. Ever since, neurolinguistic research has highly focused on the neural systems underlying sign language processing and on the modality specific role of the right hemisphere (see Corina \& Spotswood, 2012 for an overview). Interestingly, the where in the brain seems to be of much more interest than the how. That is to say, despite higher financial and organizational costs, imaging studies are much more common with sign languages than time-sensitive methods. Three recent handbook articles on sign languages and neurolinguistics each give a detailed outline of the different existing neuroimaging studies and their findings, but do not at all 
mention the few ERP studies that have been conducted so far (Emmorey, 2003; Corina \& Spotswood, 2012; Klann, 2012). What could be a reason for that? A first answer to that question could be that, at the time neuroimaging studies became possible, one of the main interests lay in the question whether sign and spoken languages are mediated in similar neural regions (Emmorey et al., 2002; Grossi, Semenza, Corazza, \& Volterra, 1996; MacSweeney et al., 2002). Furthermore, imaging studies provide a great opportunity to investigate the neural differences between language-derived manual actions (as in sign languages) and gestural-derived manual actions (as in co-speech gestures). Observations with deaf aphasic participants showed that a specific manual movement - like brushing the teeth - could not be produced in a sign language context, but could be performed as a gesture (Poizner et al., 1987). The assumption that gestural manual action emanates in neural regions distinct from those in which manual signs are computed could be supported by imaging studies (MacSweeney et al., 2004; Husain, Patkin, Thai-Van, Braun, \& Horwitz, 2009). However, although imaging studies provide an ideal method to investigate specific neural regions underlying the (sign) language system, it remains unclear why time-sensitive methods like ERP studies do not have a comparable prominence for sign language investigations as they have for spoken language investigations. One crucial aspect could be the technological and theoretical challenge to identify the exact time point according to which the electroencephalogram is analyzed. Signed sentences are ongoing streams of manual and nonmanual movements, either in lexical signs or in transitions between these signs. The theoretical challenge thus lays in identifying the "onset" of a critical target sign. The technological challenge lies in implementing this identified moment in the set-up of stimulus presentation. The following paragraph gives an overview of existing ERP studies on sign languages with respect to their way of stimulus presentation.

In 1987, the first ERP studies on sign language processing were published. One study investigated semantic anomalies in sign sentence processing (Kutas, Neville, \& Holcomb, 1987), the second investigated peripheral versus 
central visual stimulus detection (Neville \& Lawson, 1987). Ten years later, Neville et al. (1997) conducted a further ERP study on semantic mismatch in ASL sentences. They compared the processing of semantic anomalies between groups of deaf and hearing native signers, late learners and non-signers. Based on Kutas \& Hillyard's (1980) finding of an N400 for semantically unexpected words in a sentential context, the main interest in these initial studies was to investigate semantic anomalies in sign language sentences. Thus, these first studies presented signed sentences with a semantically anomalous completion. However, the technological facilities set some limits to the kind of stimulus presentation. Sentences were presented sign-by-sign, with each sign consisting of 8 digitized frames (30 ms per frame), irrespective of the natural length of the sign. Thus, they had to select "eight frames that represented the identifying movements of each sign" (Neville et al., 1997: 289). Although these studies report an N400 effect, Capek et al. (2009) can be said to be the first study that investigated semantic anomalies in ASL sentences, presented in natural signing speed. However, the paper is unspecific with regard to cutting or splicing the stimulus material. It is therefore not clear, whether participants saw the sentences in one go or with a visual interruption by a video cut. While the N400 effect for semantically incongruent signs could be replicated by Capek et al. (2009), Grosvald, Gutierrez, Hafer, \& Corina (2012), Gutiérrez, Williams, Grosvald, \& Corina (2012), and Hänel-Faulhaber et al. (2014), the stimulus sentences in these studies were presented in a somehow manipulated (i.e. cut or trimmed) manner. A further study by Gutiérrez, Müller, Baus, \& Carreiras (2012) investigated phonological form-based priming effects for the two phonological parameters handshape and location. They presented isolated signs with the video beginning at the first hold of the sign. Three further studies investigated the processing of spoken words, either in written or in auditory modality, with deaf and hearing native signers (Skotara, Kügow, Salden, Hänel-Faulhaber, \& Röder, 2011; MacSweeney, Goswami, \& Neville, 2013; Zachau et al., 2014). Accordingly, these studies presented their stimulus material in written form. 
Table 0.1 presents an overview of the existing ERP studies that were conducted with a sign language. ${ }^{1}$ Note that the research question and the results are only presented in parts. A detailed discussion of the relevant studies will take place in the upcoming chapters.

Table 0.1 Overview of existing event-related potential studies on sign languages

\begin{tabular}{|c|c|c|c|c|}
\hline $\begin{array}{l}\text { authors; } \\
\text { year }\end{array}$ & $\begin{array}{l}\text { research question; } \\
\text { language }\end{array}$ & $\begin{array}{c}\text { stimulus } \\
\text { presentation }\end{array}$ & participants & ERP results \\
\hline $\begin{array}{l}\text { Kutas et al. } \\
\text { (1987) }\end{array}$ & $\begin{array}{l}\text { semantic anomalies in: } \\
\text { (1) written English } \\
\text { sentences } \\
\text { (2) auditory English } \\
\text { sentences } \\
\text { (3) signed sentences; } \\
\rightarrow \text { ASL }\end{array}$ & $\begin{array}{l}\text { videos: sign-by-sign } \\
\text { presentation; } 1 \\
\text { sign/sec; } 8 \text { frames per } \\
\text { sign; } 30 \mathrm{~ms} \text { per frame, } \\
\text { total duration of } 240 \\
\text { ms }\end{array}$ & $\begin{array}{l}\text { (a) } 12 \text { hearing } \\
\text { subjects for (1) } \\
\text { (b) } 12 \text { different } \\
\text { hearing subjects } \\
\text { for (2) } \\
\text { (c) } 10 \text { deaf } \\
\text { native signers } \\
\text { for (3) }\end{array}$ & $\begin{array}{l}\text { for (3): centro- } \\
\text { parietal N400 } \\
(350-500 \mathrm{~ms})\end{array}$ \\
\hline $\begin{array}{l}\text { Neville \& } \\
\text { Lawson } \\
(1987)\end{array}$ & $\begin{array}{l}\text { attention to peripheral } \\
\text { vs. central located visual } \\
\text { stimuli }\end{array}$ & non-language stimuli & $\begin{array}{l}\text { congenitally } \\
\text { Deafs }\end{array}$ & $\begin{array}{l}\text { N1 component } \\
(157 \mathrm{~ms}) \\
\text { enhanced in } \\
\text { peripheral } \\
\text { location for Deafs }\end{array}$ \\
\hline $\begin{array}{l}\text { Neville et al. } \\
\text { (1997) }\end{array}$ & $\begin{array}{l}\text { semantic anomalies in } \\
\text { signed sentences; open } \\
\text { vs. closed class sigs; } \\
\rightarrow \text { ASL }\end{array}$ & $\begin{array}{l}\text { videos: sign-by-sign } \\
\text { presentation; } 8 \text { frames } \\
\text { per sign (i.e. } 30 \mathrm{~ms} \text { per } \\
\text { frame, total duration } \\
\text { of } 240 \mathrm{~ms} \text { per sign) }\end{array}$ & $\begin{array}{l}\text { (a) } 10 \text { deaf } \\
\text { native signers } \\
\text { (b) } 10 \text { hearing } \\
\text { native signers } \\
\text { (c) } 9 \text { hearing late } \\
\text { learners } \\
\text { (d) } 8 \text { hearing } \\
\text { non-signers }\end{array}$ & $\begin{array}{l}\text { - for group (a): } \\
\text { larger N400 for } \\
\text { open than closed } \\
\text { class signs } \\
\text { - N400 effect also } \\
\text { for groups (b) and } \\
\text { (c) }\end{array}$ \\
\hline $\begin{array}{l}\text { Capek et al. } \\
(2009)\end{array}$ & $\begin{array}{l}\text { (1) semantic anomalies } \\
\text { in signed sentences; } \\
\text { (2) verb agreement } \\
\text { violation in signed } \\
\text { sentences; } \\
\rightarrow \text { ASL }\end{array}$ & $\begin{array}{l}\text { videos: ASL sentences } \\
\text { were videotaped and } \\
\text { presented at natural } \\
\text { signing rate; whether } \\
\text { the material was } \\
\text { spliced remains } \\
\text { unclear. }\end{array}$ & $\begin{array}{l}15 \text { deaf native } \\
\text { signers }\end{array}$ & $\begin{array}{l}\text { (1) N400 effect } \\
(300-875 \mathrm{~ms}) \\
\text { (2) early anterior } \\
\text { negativity + P600 } \\
\text { (see Chapter } 3 \text { for } \\
\text { discussion) }\end{array}$ \\
\hline
\end{tabular}

${ }^{1}$ This table has no claim to completeness and represents the review of my literature search. There is one further ERP study on the neural correlates of syntactic processing in Israeli Sign Language, a masters thesis that is only available in the National Library of Israel, Jerusalem $(\mathrm{Oz}, 2007)$.

The ERP study investigating the electrophysiology of vegetable language (Frisch \& beim Graben, 2007) was not included in this list. This is due to the fact that the authors remain unclear about the sign language knowledge of the participating pumpkin. 


\begin{tabular}{|c|c|c|c|c|}
\hline $\begin{array}{l}\text { Skotara et al. } \\
\text { (2011) }\end{array}$ & $\begin{array}{l}\text { written German } \\
\text { sentences with: } \\
\text { (1) semantic violation } \\
\text { (implausible object) or } \\
\text { (2) verb agreement } \\
\text { violation } \\
\rightarrow \text { German }\end{array}$ & $\begin{array}{l}\text { written words: word- } \\
\text { by-word presentation; } \\
600 \mathrm{~ms} \text { per word }\end{array}$ & $\begin{array}{l}\text { (a) } 8 \text { deaf native } \\
\text { signers, German } \\
\text { L2 learners } \\
\text { (b) } 12 \text { hearing } \\
\text { German L2 } \\
\text { learners } \\
\text { (c) } 12 \text { hearing } \\
\text { German native } \\
\text { speakers }\end{array}$ & $\begin{array}{l}\text { for group (a): } \\
\cdot \text { N400 effect } \\
(300-500 \mathrm{~ms}) \text { and } \\
\text { late positivity } \\
(600-800 \mathrm{~ms}) \text { for } \\
\text { semantic violation } \\
\cdot \text { P600 (600- } \\
800 \mathrm{~ms}) \text { for } \\
\text { agreement viol. }\end{array}$ \\
\hline $\begin{array}{l}\text { Grosvald } \\
\text { et al. (2012) }\end{array}$ & $\begin{array}{l}\text { signed sentences with } \\
\text { semantic anomalies: } \\
\text { unexpected sign, } \\
\text { pseudo-sign, or } \\
\text { grooming gesture } \\
\rightarrow \text { ASL }\end{array}$ & $\begin{array}{l}\text { videos: sentence frame } \\
\text { and ending item were } \\
\text { recorded separately, } \\
\text { and edited together; } \\
\text { transitional } \\
\text { movements between } \\
\text { sentences and endings } \\
\text { were trimmed. }\end{array}$ & $\begin{array}{l}16 \text { deaf signers } \\
(11 \text { native, } 5 \\
\text { non-native })\end{array}$ & $\begin{array}{l}\cdot \mathrm{N} 400 \text {-like } \\
\text { response for } \\
\text { unexpected and } \\
\text { pseudo-signs } \\
\text { - large positivity } \\
\text { for grooming } \\
\text { gestures }\end{array}$ \\
\hline $\begin{array}{l}\text { Gutiérrez et } \\
\text { al. (2012a) }\end{array}$ & $\begin{array}{l}\text { phonological } \\
\text { processing: form-based } \\
\text { priming in either } \\
\text { handshape or location } \\
\text { parameter } \\
\rightarrow \text { LSE }\end{array}$ & $\begin{array}{l}\text { videos: single sign } \\
\text { presentation (from } \\
\text { sign onset to sign } \\
\text { offset); "onset" = 1st } \\
\text { stable frame with } \\
\text { initial location and } \\
\text { handshape; "offset" = } \\
\text { last hold of the sign } \\
\text { (frame unclear) }\end{array}$ & $\begin{array}{l}\text { (a) } 10 \text { deaf } \\
\text { native signers } \\
\text { (b) } 10 \text { deaf non- } \\
\text { native signers }\end{array}$ & $\begin{array}{l}\cdot \text { location } \\
\text { priming: higher } \\
\text { amplitude of } \\
\text { N400 for signs } \\
\cdot \text { late negativity } \\
\text { effect for non- } \\
\text { signs }\end{array}$ \\
\hline $\begin{array}{l}\text { Gutiérrez et } \\
\text { al. }(2012 b)\end{array}$ & $\begin{array}{l}\text { sentence processing, } \\
\text { interaction between } \\
\text { semantic relation }(+\mathrm{S} / \\
-\mathrm{S}) \text { and phonological } \\
\text { overlap }(+\mathrm{P} /-\mathrm{P}) \\
\text { compared with expected } \\
\text { baseline sentence } \\
\rightarrow \text { ASL }\end{array}$ & $\begin{array}{l}\text { videos: sentence } \\
\text { frames, target items } \\
\text { and endings were } \\
\text { recorded separately } \\
\text { and trimmed later on }\end{array}$ & $\begin{array}{l}17 \text { deaf native } \\
\text { signers }\end{array}$ & $\begin{array}{l}\cdot \text { early negativity } \\
(150-250 \mathrm{~ms}) \text { for } \\
\text { semantically } \\
\text { related }(+\mathrm{S} /-\mathrm{P}) \\
\text { and }(+\mathrm{S} /+\mathrm{P}) \\
\cdot \mathrm{N} 400(350- \\
450 \mathrm{~ms}) \text { for }(+\mathrm{S} / \\
-\mathrm{P}) \text { and }(-\mathrm{S} /+\mathrm{P}) \\
\cdot \text { central } \mathrm{N} 400 \\
(450-600 \mathrm{~ms}) \text { for } \\
\text { all conditions } \\
\text { compared to } \\
\text { baseline }\end{array}$ \\
\hline $\begin{array}{l}\text { MacSweeney } \\
\text { et al. (2013) }\end{array}$ & $\begin{array}{l}\text { English word rhyme } \\
\text { task (phonol. rhyme, not } \\
\text { orthographic) with } \\
\text { hearing and deaf } \\
\text { participants; } \\
\rightarrow \text { English }\end{array}$ & $\begin{array}{l}\text { written words: word- } \\
\text { by-word presentation; } \\
500 \mathrm{~ms} \text { per word }\end{array}$ & $\begin{array}{l}\text { (a) } 9 \text { deaf native } \\
\text { signers (ASL) } \\
\text { (b) } 9 \text { hearing } \\
\text { monolingual } \\
\text { English speakers }\end{array}$ & $\begin{array}{l}\text { negativity (300- } \\
600 \mathrm{~ms} \text { ) for } \\
\text { unrhymed targets, } \\
\text { for both groups }\end{array}$ \\
\hline $\begin{array}{l}\text { Hänel- } \\
\text { Faulhaber } \\
\text { et al. (2014) }\end{array}$ & $\begin{array}{l}\text { (1) semantic anomalies } \\
\text { in signed sentences, and } \\
\text { (2) verb agreement } \\
\text { violation in signed } \\
\text { sentences } \\
\rightarrow \text { DGS }\end{array}$ & $\begin{array}{l}\text { videos: continuous } \\
\text { sentences, including } \\
\text { transition phases } \\
\text { (trigger positions); } \\
\text { whether the material } \\
\text { was spliced remains } \\
\text { unclear }\end{array}$ & $\begin{array}{l}11 \text { deaf native } \\
\text { signers }\end{array}$ & $\begin{array}{l}- \text { Semantic } \\
\text { violation: N400 } \\
(550-750 \mathrm{~ms}) \\
\cdot \text { Agreement } \\
\text { violation: LAN } \\
(400-600 \mathrm{~ms}) \text { and } \\
\text { P600 (1000- } \\
1300 \mathrm{~ms})\end{array}$ \\
\hline
\end{tabular}




\begin{tabular}{|c|c|c|c|c|}
\hline $\begin{array}{l}\text { Zachau et al. } \\
\text { (2014) }\end{array}$ & $\begin{array}{l}\text { Semantic decision task } \\
\text { with prime-target pairs, } \\
\text { either both in Finish or } \\
\text { Finish prime and FinSL } \\
\text { target. } \\
\rightarrow \text { Finish, FinSL }\end{array}$ & $\begin{array}{l}\text { videos: single sign } \\
\text { presentation (from } \\
\text { resting to resting } \\
\text { position); } \\
\text { "onset" and "offset" } \\
\text { were defined as hands } \\
\text { began to move and } \\
\text { ended in resting } \\
\text { position. }\end{array}$ & $\begin{array}{l}\text { (a) } 15 \text { hearing } \\
\text { native signers } \\
\text { (CODAs) } \\
\text { (b) } 13 \text { hearing } \\
\text { FinSL late } \\
\text { learners } \\
\text { (interpreters) } \\
\text { (c) } 15 \text { hearing } \\
\text { non-signers } \\
\text { (controls) }\end{array}$ & $\begin{array}{l}\text { N400 and Late } \\
\text { Positivity } \\
\text { Complex for } \\
\text { signed and } \\
\text { auditory targets } \\
\text { (after tCPA } \\
\text { application) }\end{array}$ \\
\hline
\end{tabular}

As becomes apparent in Table 0.1 under the column "stimulus presentation", the way in which stimulus videos were presented advanced with the technological capabilities. However, it seems that none of these studies presented their stimulus sentences in a non-manipulated video, that is, a video that does not contain a visual detectable cut or trim. A crucial difficulty for ERP studies with sign languages seems to be the identification of the critical moment to which the electrophysiological signal is analyzed, the so-called trigger position. In ERP studies with spoken languages presented in the visual modality (i.e. in written form), the onset of a target word can be defined as the moment at which the word appears on the computer screen. In ERP studies presenting auditory sentences - a method that is more similar to presenting natural sign language videos - the onset of the critical word can be identified by minor pauses between the words. The sentences can be digitally cut into word pieces and triggers can be aligned with the onsets of those pieces (Holcomb \& Neville, 1991). However, ERP studies with sign languages face the challenge that transitions between lexical signs are rather smooth than clear-cut. Transition phases themselves are dynamic and the manual movements of hands and arms are similar in speed and direction compared to lexical signs. Also, the changes between the phonological parameters of one sign to the next (i.e. changes in handshape, orientation, location, and/or movement) are fluent and extend to several video frames. Thus, it is a theoretical challenge to define the criteria of the onset of a sign within the continuous signing stream (Jantunen, 2013, 2015). A conventional solution is to cut the video stream and time-align the trigger position with the spliced or trimmed video sequence of the target sign. This procedure, however, 
interrupts the naturalness of the ongoing signing stream and might cause an interference of the ongoing processing of the sentence.

Chapter 1 addresses this question in detail and presents a methodological solution. By presenting non-manipulated videos of signed sentences recorded in one go, we expanded the methodological conventions and focused on the maximal naturalness of the stimulus material. This put a challenge to the deaf informants who signed the stimulus sentences. In order to reduce small differences between one sentence and its corresponding counterpart across conditions, the informants had to be very accurate in their way of articulation. Nevertheless, the use of non-manipulated videos enables us to investigate the processing of linguistic aspects within the ongoing processing of the whole sentence.

Apart from the methodological need to identify the crucial trigger positions within the ongoing signing stream, ERP studies with sign languages (in Germany) face further challenges: Sign language education has a problematic history in Germany and Europe. Until the beginning of the $21^{\text {st }}$ century, the oral method was predominantly used in schools for deaf children and still has an impact on the education of deaf children nowadays (c.f., McBurney, 2012). Also, only a subgroup of deaf people are born to deaf parents (about 10\% according to the Deutscher Gehörlosen Bund e.V.),, ${ }^{2}$ and thus has the opportunity to learn German Sign Language as native language. In Germany, therefore, deaf adults are a very heterogeneous group with respect to their age of sign language acquisition, their age of spoken and written language acquisition, and their educational background. Thus, it is plausible to compare the group of Deaf ${ }^{3}$ to so-called non-WEIRD subjects, i.e. subjects that do not typically come from Western, Educated, Industrialized, Rich, and Democratic societies (Henrich, Heine, \& Norenzayan, 2010a, 2010b). Of course, deaf people in Germany grow up in a western, industrialized, rich and

2 C.f., http://www.gehoerlosen-bund.de/index.php?option=com_content\&view=cate gory\&layout=blog\&id=38\&Itemid=101\&lang=de, accessed on Oktober 17th 2014.

${ }^{3}$ As a convention, upper case 'Deaf' refers to users of DGS who are members of the Deaf community, while lower case 'deaf' refers to the audiological state of deafness. 
democratic country, and they are educated and have the opportunity to study at a University, in principle. But, to study at a German University is much more difficult for a deaf person then for a hearing person, or than it is for a deaf person in the United States studying at Gallaudet University, Washington D.C., the only university worldwide that provides their program in a sign language. The age and the way of sign and spoken language acquisition has a crucial impact on the functional organization of both language modalities in the brain (Mayberry, 2007; Malaia \& Wilbur, 2010; Mayberry, Chen, Witcher, \& Klein, 2011). Thus, in order to control for this confounding variable, only participants who had deaf parents and learned DGS from birth on, or who learned DGS before the age of three participated in the studies presented here.

The methodological approach is one of the main linking factors between the three individual studies in this thesis. Although each study investigates a different linguistic aspect, these are explored in the context of processing whole sentences, presented in non-manipulated videos. Exploring linguistic aspects within whole sentence processing comes closer to natural language processing (as we do in our everyday life) than, for example, single word or single sign processing (Bornkessel-Schlesewsky, Staub, \& Schlesewsky, n.d.). Since all sentences were created together with Deaf informants, any minor disparities from natural sentences derive from the experimental design. The methodological extension to analyze ERP correlates in relation to different time-locking information points during the signing stream is a further joined aspect of these studies that enables the achievement of unforeseen results in contrast to conventional methods.

Sign language research is at a crucial point of development. After a period of time in which the status of sign languages had to be proven as natural languages, now the modality-specific aspects of sign languages come to the fore more strongly. But even the modality-specific properties of sign languages (as for example, sign language agreement) are described with respect to linguistic terms that originated from spoken language linguistics. 
The following studies do not have the aim of breaking with classic linguistic terms, nor do they aim to introduce a research approach totally distinct from spoken languages. Rather, they want to offer a perspective on the processing of a sign language detached from what we expect when coming from spoken languages. Hence, the three following chapters present separate studies that investigate individual research questions. There is no overall theoretical frame that tries to explain a general theory or model on processing sign language sentences. Instead, the studies cover a large content spectrum from sub-lexical and phonological to morpho-syntactic and semantic aspects of sign language processing. Thus, each chapter stands for itself. All relevant references, as well as the interpretation of the results, are discussed within each chapter.

The first chapter presents a classic semantic expectation mismatch design and addresses the importance of transition phases for the processing of signs based on lexical prediction via forward models. Transition phases between one sign and the next are relatively long and external from the body of the signer. This enables the addressee to set up expectations on the movement trajectory or hand configuration of the upcoming sign, although semantic information during the transition phase is minimal. Under the assumption of a forward model, we asked whether an expectation mismatch is already measurable prior to the lexical sign onset. Thus, in the first chapter, the focus is on sub-lexical properties of sign languages and their relevance for the anticipation of an upcoming sign.

The second chapter focuses on cross-modal cross-language co-activation. In this ERP study, we investigate overt phonological priming in DGS and covert orthographic priming of German sign translations. The processing of phonologically minimal pairs in DGS, as well as prime-target pairs that have an orthographic overlap in their German translation, is investigated within the processing of a DGS sentence. This chapter addresses two interesting aspects: The minor aspect is the relevance of phonological parameters for sign processing. The four phonological parameters each sign is constituted of 
are realized simultaneously. For theories on lexical access in sign languages, this constitutes a challenge. Several studies have investigated the different impact of, for example, location or handshape on the lexical access of a sign (Dye \& Shih, 2006; Gutiérrez, Müller, et al., 2012). This chapter presents an ERP study on overt phonological priming in DGS. The more crucial aspect follows the question how language co-activation in bimodal bilinguals is mediated. Deaf people grow up in a hearing environment and constantly navigate between sign language and spoken (written) language communication. Thus, it is likely that both languages are continuously activated. However, since there is no overt phonological similarity between both language modalities, language co-activation in congenitally profoundly deaf people raises the question on what bases the two languages are linked. This pertains to the question of what kind of phonological or sub-lexical representation deaf people have of spoken words, since acoustic information is not accessible to them. Chapter 2 addresses these questions and discusses possible explanations in the light of a neurolinguistic perspective on phonological aspects during sentence processing.

The third chapter deals with a theoretically highly discussed topic in sign language research: agreement. As outlined in the special issue of Theoretical Linguistics - "On the linguistic status of 'agreement' in sign languages" (LilloMartin \& Meier, 2011), agreement in sign languages exhibits some modalityspecific properties that cannot be found in agreement systems of spoken languages. Agreement in sign languages is expressed via a location overlap of the beginning and/or ending of the verb with the location associated with its arguments (i.e. subject and/or object). Whether this phenomenon can be compared to concatenational agreement affixes used in spoken languages is discussed with the results of two ERP studies on agreement violation with agreement verbs and agreement violation with plain verbs. Plain verbs provide the unique opportunity to test the agreement principle on a group of verbs that are (not yet) specified for location agreement. Although previous ERP studies on agreement violation in sign languages report similar electrophysiological effects to studies on agreement violation in spoken languages, 
and thus highlight the morpho-syntactic status of sign language agreement, Chapter 3 questions these findings and discusses alternative interpretations of agreement.

The thesis is structured as follows: Each chapter is treated as an individual paper. Therefore, the relevant terms, as well as the related references and topics are introduced and discussed within each chapter. The structure of each chapter is analogous. Section X.1 gives an introduction into the topic, outlines the pertinent literature and presents the design of each study. Section X.2 describes in detail the methodological aspects of each study, regarding the subject groups, the stimulus material, the EEG recording, and the statistical analysis. Section X.3 of each chapter presents the results, followed by Section X.4, in which these are discussed and related to previous findings. Section X.5 completes each chapter with a short summary and conclusion. An overall synopsis including a short outlook on the further research direction is provided in the final "Conclusion". 


\title{
1 Lexical prediction via forward models: N400 evidence from German Sign Language
}

\begin{abstract}
Models of language processing in the human brain often emphasize the prediction of upcoming input - for example in order to explain the rapidity of language understanding. However, the precise mechanisms of prediction are still poorly understood. Forward models, which draw upon the language production system to set up expectations during comprehension, provide a promising approach in this regard. Here, we present an event-related potential (ERP) study on German Sign Language (DGS), which tested the hypotheses of a forward model perspective on prediction. Sign languages involve relatively long transition phases between one sign and the next, which should be anticipated as part of a forward model-based prediction even though they are semantically empty. Native speakers of DGS watched videos of naturally signed DGS sentences, which either ended with an expected or a (semantically) unexpected sign. Unexpected signs engendered a biphasic N400 - late positivity pattern. Crucially, N400 onset preceded critical sign onset and was thus clearly elicited by properties of the transition phase. The comprehension system thereby clearly anticipated modality-specific information about the realization of the predicted semantic item. These results provide strong converging support for the application of forward models in language comprehension.
\end{abstract}

\subsection{Introduction}

The literature on the neurophysiology of language has recently seen a great deal of discussion with regard to the role of prediction in language processing. Thus, there is good evidence to suggest that the human language processing system anticipates individual words during the comprehension process. For example, DeLong, Urbach, \& Kutas, (2005) observed a modulation of the N400 event-related brain potential (ERP) when a determiner ("a" or "an") was incompatible with the predicted following noun (e.g. when "kite" was predicted as in "The day was breezy so the boy went outside to fly ...", "an" versus "a" engendered an N400 effect). Findings such as these (for similar results, see Otten, Nieuwland, \& van Berkum, 2007; Van Berkum, 
Brown, Zwitserlood, Kooijman, \& Hagoort, 2005; Wicha, Moreno, \& Kutas, 2004) provide strong converging support for the assumption that the language processing system actively engages in predictive processing of upcoming input, rather than relying primarily on bottom-up input information (for a framework describing the interplay between top-down prediction and bottom-up information, see Federmeier, 2007).

\subsubsection{Modeling prediction in language processing}

How should these predictive mechanisms be envisaged? Perhaps the most straightforward assumption in this regard is that prediction is implemented via lexical preactivation. In this view, the sentence (and discourse) context serves to preactivate expected (or lexically associated) upcoming words and the degree of a word's preactivation determines the N400 amplitude. Such "lexical" accounts of the N400 have become dominant over the past few years, as they can derive the observation that the N400 does not straightforwardly mirror sentence plausibility (Brouwer, Fitz, \& Hoeks, 2012; Lau, Phillips, \& Poeppel, 2008; Stroud \& Phillips, 2012). This is apparent, for example, in "semantic reversal anomalies" such as "The hearty meals were devouring ..." (Kim \& Osterhout, 2005) - i.e. implausible sentences in which the critical word has a high degree of lexical-semantic association to the preceding context and which do not engender an N400 effect in comparison to plausible controls in English and Dutch (e.g., Hoeks, Stowe, \& Doedens, 2004; Kim \& Osterhout, 2005; Kolk, Chwilla, van Herten, \& Oor, 2003; Kuperberg, Sitnikova, Caplan, \& Holcomb, 2003). ${ }^{4}$ In spite of their inherent appeal, however, lexical models of this type do not provide a principled explanation for N400 amplitude modulations that are not due to spreading activation

4 Note, however, that this may be a language-specific phenomenon, as other languages such as German, Chinese and Turkish do show N400 effects for semantic reversal anomalies (Bornkessel-Schlesewsky et al., 2011). In addition, recent results from English indicate that N400 effects for reversal anomalies can vary even within a language based on the experimental environment (Bourguignon, Drury, Valois, \& Steinhauer, 2012). 
between lexical entries (for N400 effects based on discourse congruence independently of lexical association, see Camblin, Gordon, \& Swaab, 2007). This raises the question of how more abstract levels of prediction might be implemented.

The assumption of forward models in language processing appears to provide a promising solution to this question. As proposed by Pickering \& Garrod (2007), the language comprehension system may draw upon the language production system to emulate (i.e. set up a forward model of) the current input. The output of this model, i.e. the predicted word, can then be matched against the word actually encountered. In the neurophysiological domain, a similar view has been advocated by Federmeier (2007). She proposes that top-down, predictive mechanisms in language comprehension are achieved via a tight coupling between the comprehension and production systems and that this coupling takes place primarily within the left hemisphere. The right hemisphere, by contrast, processes the input in a more strongly stimulusbased (feed-forward) manner. Evidence for this view stems from ERP studies with visual half-field presentation techniques (for an overview, see Federmeier, 2007) and from correlations between production measures and predictive processing in comprehension (Federmeier, Kutas, \& Schul, 2010). An interdependence between the N400 and production abilities has further been reported for commissurotomy patients (Kutas, Hillyard, \& Gazzaniga, 1988).

It remains to be examined, however, how specific the information provided by such forward models is. The vast majority of previous electrophysiological studies on prediction in language comprehension have used segmented (typically word-by-word) visual presentation. Hence, to explain prediction under these conditions, a forward model would essentially only need to provide an activated lexical entry and, perhaps, a visual word form (for evidence in favor of prediction down to the orthographic level, see Dikker \& Pylkkänen, 2011; Dikker, Rabagliati, Farmer, \& Pylkkänen, 2010). In accordance with current neurobiological models of speech processing, however, 
forward models could also be expected to provide much more detailed information regarding the projected upcoming input. Rauschecker \& Scott (2009, p. 722), for example, assume a "predictive motor signal" that "inform[s] the sensory system of motor articulations that are about to happen". In the present study, we tested the hypothesis that forward models in language processing go beyond the activation of lexical entries and, instead, provide modality-specific information regarding the expected sensory properties of the upcoming input. To this end, we capitalized upon the manual-visual modality of sign languages. Sign languages have modalityspecific articulatory properties, which render them an ideal testing ground for examining the specificity of forward models in language processing. In the next subsection, we briefly introduce two properties that will be most relevant for our study.

\subsubsection{Predicting input in a sign language: Simultaneity and three-dimensionality}

Because of the specific properties of the manual-visual modality, sign languages differ from spoken languages in two respects: First, they are produced in a three-dimensional signing space and second, they can use different kinds of articulators simultaneously. These articulatory differences also affect the architecture of grammar. The three-dimensional signing space in front of the signer's upper body is relevant for the production of lexical manual signs, which are constituted by the four basic phonological parameters handshape, orientation, location, and movement. A change of one parameter in any of the three spatial dimensions can cause a change in meaning (Brentari, 1998; Padden \& Perlmutter, 1987; Sandler, 1989; Stokoe, 1960). (Note that orientation is not always treated as a fourth parameter but often subsumed together with handshape under the term "hand configuration" (Battison, 1978; Sandler \& Lillo-Martin, 2006). We list them separately here because many sign languages show phonological minimal pairs for both parameters. Note, however, that the distinction between handshape and 
orientation is not relevant for our study. Therefore, we also use the term "hand configuration" when the distinction between orientation and handshape is irrelevant for the purposes of the discussion.) For example, the minimal pair GIVE and VISIT in German Sign Language (DGS) only differs in the orientation of the palm: upwards (supine) versus inwards (neutral). In all other parameters, the two signs are completely identical. Additionally, sign languages have the opportunity to use multiple distinct articulators simultaneously (fingers, hands and arms for manual signs; and face, head, and upper part of the body for so-called non-manual components). This use of multiple articulators enables the simultaneous realization of lexical and grammatical information manually and non-manually (Pfau \& Quer, 2010; Wilbur, 2000). On a sub-lexical level, for instance, all four phonological parameters are produced simultaneously to realize a lexical sign. While the hand is shaped in a certain form, palm and fingers are oriented into a certain direction, and hand and arm are positioned at a certain location before moving on a lexically (or grammatically) specified path. None of these parameters can be articulated independently from the others, as will be discussed in more detail in Section 1.1.5. The specific properties of the manual-visual modality thus enable sign languages to realize phonological parameters simultaneously.

Crucially for the purposes of the present study, a continuous signing stream must involve transitions between the phonological parameters of one sign and those of the following sign. This divides the signing stream into two kinds of phases: lexical signs and transition phases between signs. Interestingly, in sign languages, unlike in spoken languages, these transition phases between signs are rather long, due to the relatively massive articulators, which have to move in space (Meier, 2002). Therefore, sign languages are an ideal object of study for time-sensitive experimental methods. In the present study, we examined ERP correlates of processing these transition phases in order to shed light on whether the language processing system sets up specific predictions regarding hand trajectories and change of hand configuration towards an expected sign. Under the assumption of a forward model that 
allows for the anticipation of modality-specific sensory properties of the linguistic input, we hypothesize that prediction error should already be measurable within the (non-lexical) transition phase (i.e. prior to the critical sign onset).

\subsubsection{Previous electrophysiological studies on sign language processing}

Event-related potential studies on the processing of natural signing have been very rare up to now. (Kutas et al., 1987) were the first to show that N400 effects for semantic anomalies occur in written, spoken and signed contexts. This general modality independence was subsequently confirmed by further studies on lexical-semantic aspects of sign language processing in American Sign Language (Neville et al., 1997; Capek et al., 2009; Grosvald et al., 2012; Gutiérrez et al., 2012a; Gutiérrez et al., 2012b) and additionally extended to syntactic processing (Capek et al., 2009).

The relationship between the $\mathrm{N} 400$ and sign recognition was further investigated by Grosvald et al. (2012). In an ERP-experiment, they compared the processing of (contextually expected and unexpected) linguistic signs to the processing of possible but non-existing pseudo-signs and non-linguistic "grooming gestures" (e.g. rubbing one's eye). While both unexpected signs and non-existing pseudo-signs showed an N400 effect, the non-linguistic grooming gestures engendered a broad positivity after approximately $600 \mathrm{~ms}$. Grosvald and colleagues thus concluded that grooming gestures are rapidly identified as non-linguistic material and thereby analyzed differently to (potentially meaningful) signs.

Additionally, a recent ERP study on Spanish Sign Language (LSE) attests to the importance of location in sign recognition. In an ERP priming paradigm using a lexical-decision task, Gutiérrez et al. (2012a) compared the effects of handshape and location on the lexical access of signs. Targets either shared the same location, the same handshape or were unrelated to the preceding 
prime. Gutiérrez and colleagues found a location-based N400 priming effect for existing signs, but no handshape-related priming. From these results, they concluded that the parameter handshape is not as relevant as the parameter location for sign recognition. For additional results on the relevance of location in sign recognition, see also behavioral studies with deaf adults (cf. Carreiras, Gutiérrez-Sigut, Baquero, \& Corina, 2008; Orfanidou, Adam, McQueen, \& Morgan, 2009), as well as studies on sign language acquisition, language impairment and slips of the hand (cf. Corina, 2000; Hohenberger et al., 2002; Marentette \& Mayberry, 2000; Morgan, Barrett-Jones, \& Stoneham, 2007).

While these previous studies have provided important initial insights into the neural processing of sign languages, they all have in common that they focused exclusively on sign onsets. Thus, there is no study to date that has reported neurophysiological correlates of transition phase processing. Indeed, in the vast majority of studies, transition phases were excluded from the stimuli (via modifications of the video material); in one study (Capek et al., 2009), it is not clear whether transitions were included in the materials, but no ERP correlates of transition processing are reported. Since transition phases provide important linguistic information for the processing of sign languages, it appears crucial to include and analyze this information when examining event-related potential correlates of natural sign language comprehension. Otherwise, the presented linguistic material is either highly artificial (if the transition phases were cut out) or important information is missing (if event-related potentials are only examined relative to critical sign onset, i.e. following the end of the transition phase to the critical item). In the present study, we report, for the first time, a detailed analysis of ERP effects engendered in response to the transition phase within natural sign language processing. 


\subsubsection{The present study}

The present study aimed to test the hypothesis - outlined in Section 1.1.2 that forward models in language processing supply specific, modalitydependent information regarding the sensory properties of the upcoming input. To this end, it examined whether prediction-based N400 effects to unexpected versus expected sentence-final verbs in DGS can already be measured within the transition phase to the critical sign (i.e. before sign onset). If such effects can indeed be observed, they would provide strong converging support for the assumption that forward models go beyond the preactivation of (amodal) lexical entries and rather provide specific expectations about the motor program by means of which the upcoming input will be executed.

We used a semantic expectation mismatch design with two conditions. In the expected condition, the final verb of a continuous DGS sentence was semantically expected (e.g. 1a). ${ }^{5}$ In the unexpected condition, by contrast, the final verb was a possible but highly unexpected continuation (e.g. 1b).

(1) a. Expected continuation:

WOODS INDEX PATH RABBIT JUMP

'In the woods, a rabbit jumps across a path.'

b. Unexpected continuation:

ZOO INDEX RHINO BREAD JUMP

'In the zoo, a rhino jumps across some bread.'

In contrast to previous ERP studies on sign language processing, we varied the sentential context between the two conditions instead of the critical verb.

\footnotetext{
${ }^{5}$ By convention, signs are glossed using small caps. INDEX is a pointing sign, which is used for localizing non-present discourse referents at (referential) loci in the signing space and in pronominalization. Note that DGS is a verb-final language such that the examples provided here adhere to normal word order.
} 
Moreover, all critical items were so-called spatial classifier verbs which were signed in neutral signing space expressing the movement or location of an entity in topographic space (for spatial classifier verbs see Benedicto \& Brentari, 2004; Zwitserlood, 2003, 2012). This enabled us to compare ERPs to identical signs (used once in an expected and once in an unexpected context) and trajectories with an identical destination in the neutral signing space as well as handshape and orientation changes during the preceding transition phase.

In view of the crucial role of the transition phase for the aims of the present study, we will discuss the properties of transition phases in sign language in a little more detail before going on to describe the experimental methods. Manual signs are essentially a combination of two kinds of phases: movement-phases, in which hand(s) and arm(s) describe a movement path; and static phases, in which the hand configuration (a certain handshape with a certain orientation) stays relatively still at one location in signing space. In the following, we refer to static phases as "holds" and movement phases as "movements" (cf. Liddell \& Johnson, 1989). (Note that, with the use of this terminology we do not presuppose a specific model of sign language phonology. Here, we only use the terms "hold" and "movement" to describe the phonological properties of the stimulus material. For an overview of different phonological theories see Brentari, 2012.) Transition phases between two signs begin with the end of the final hold (f-hold) of the previous sign and end with the beginning of the initial hold (i-hold) of the following sign. Depending on the number of phonological parameters that need to be changed, the transition phase can include a maximum of three different phonological adaptations: a change in handshape, a change in orientation, and a trajectory between the f-hold and the i-hold. The trajectory is the three-dimensional path in signing space described by the hands when moving from one location to another. Although these parameters cannot be realized individually (i.e. each transition phase manifests a particular change of handshape, orientation and trajectory), each parameter could change independently of the other two. In our stimuli we found two kinds of adaptations. First, the change of location 
describes a three dimensional trajectory between the location of the f-hold and the target location of the i-hold. Second, the change of hand configuration (i.e. the combination of the phonological parameters handshape and orientation, see Section 1.1.2) starts with relaxing the fingers to a moment of complete loss of tension, followed by selecting the fingers of the target handshape, all accompanied by a twist of the hand orientation. Figure 1.1 provides a schematic illustration of the transition phase between two adjacent signs, indicating the trajectory between the final location (f-hold) and the target location (i-hold). ${ }^{6}$

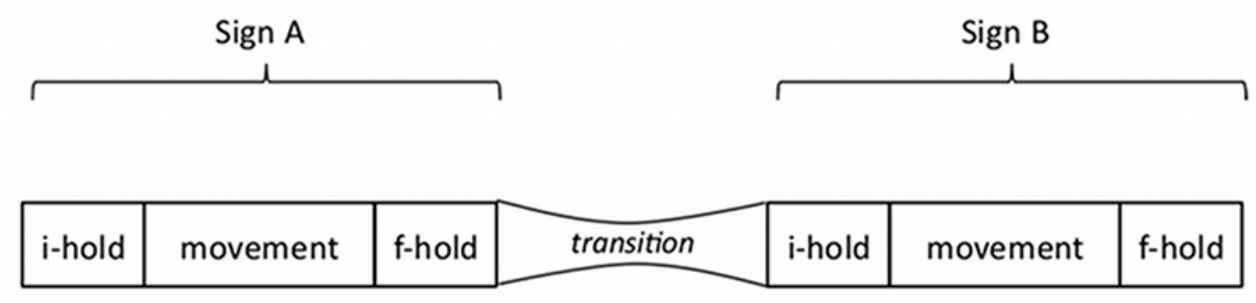

Figure 1.1 Schematic illustration of the transition phase between two signs.

As is apparent from Figure 1.1, the transition phase between signs is a fluent change of phonological parameters (zero to maximally three changes). The two adaptation processes - the change of location and the change of hand configuration - evolve simultaneously but do not depend on each other.

Figure 1.2 illustrates the trajectory of a location change. Crucially, trajectories are not arbitrary but rather depend on the relative positions of the previous sign and the target sign, and follow certain economical principles such as least effort. As shown for a right-hander in Figure 1.2, the sign RABBIT in DGS is produced at the right forehead, while the following sign JUMP is produced on

${ }^{6}$ Note that both holds vary in their duration between 40 and 120 ms. Thus, compared to the movement phase, which can last over a second, holds are relatively short. 
the right hand side in neutral space (the ipsilateral area of the signing space). Thus, the trajectory in the transition phase starts at the right forehead and moves via the shortest distance to the ipsilateral area in neutral space.

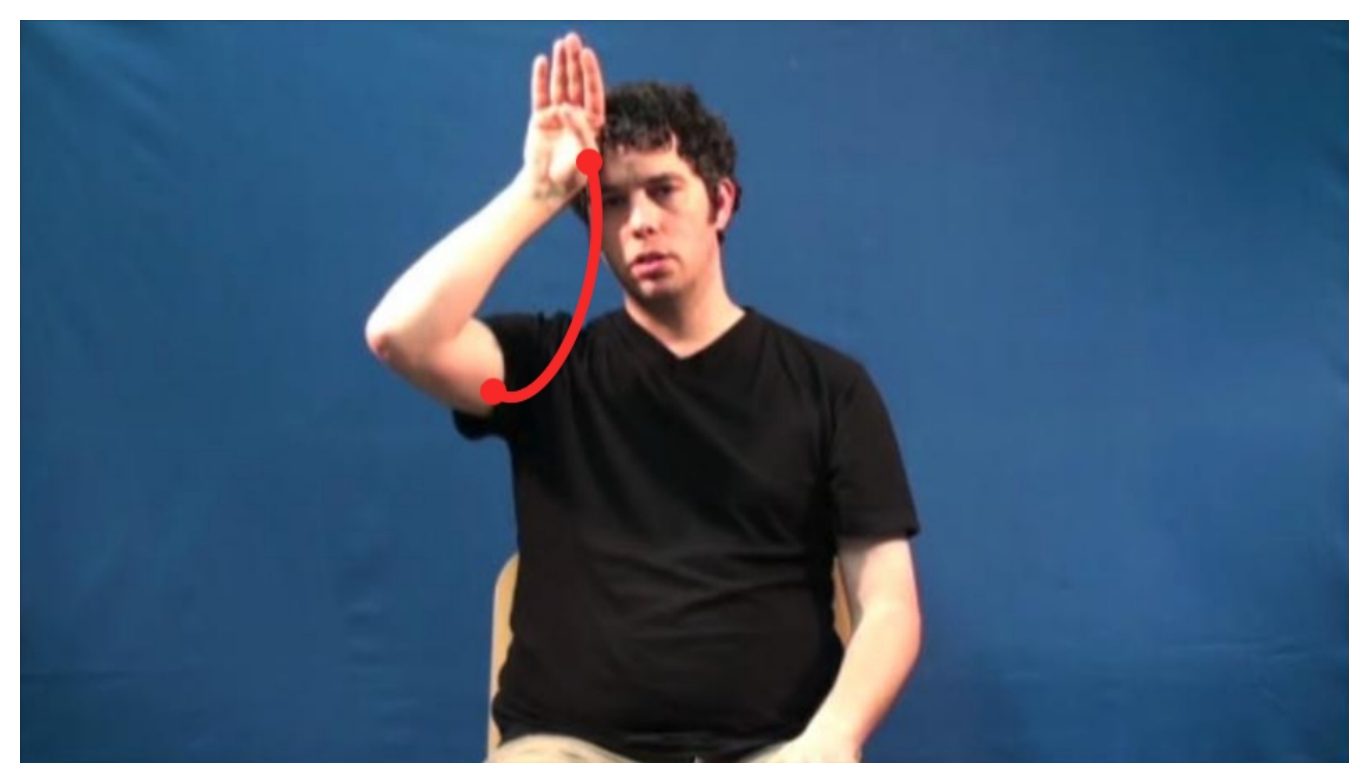

Figure 1.2 Trajectory for the change of location between the previous sign RABBIT and the following sign JUMP.

In accordance with the considerations outlined above, any transition between two given locations (e.g. 'forehead' to 'right side of neutral space') should show a similar trajectory, and thus be predictable. In addition to the change of location, the hand configuration also changes during the transition phase. The signer first relaxes the fingers used to produce the handshape of the sign RABBIT (i.e. the so-called B-hand) and then selects and bends the two fingers necessary to produce the sign JUMP (i.e. the index and the middle finger). In addition, a slight change of orientation towards the contralateral area of the singing space occurs because the target sign JUMP is produced with a movement from right to left. 
Assuming a detailed, modality-specific forward model as hypothesized above, the trajectory towards a critical sign and the specific change in hand configuration should thus form part of the predictive information supplied by the forward model. If this is the case, we should expect to observe expectation-related modulations of the $\mathrm{N} 400$ in response to changes in phonological parameters within the transition phase and thus prior to critical sign onset. Furthermore, time-locking ERPs to different information sources available at different points in time within the transition phase (e.g. handshape change, target handshape or sign onset of the target sign), will enable us to detect the time-point at which the human language processing system anticipates the phonological mismatch with the expected sign.

\subsection{Materials and methods}

\subsubsection{Participants}

A total of 20 congenitally deaf native signers of DGS participated in this experiment as paid volunteers (12 male, 8 female) after giving written informed consent. All participants were right-handed, had normal or corrected-tonormal vision and reported no history of neurological disorders. Their ages ranged from 18 to 51 years (mean age: 36). All signers had deaf parents or DGS input before the age of three. Two participants were excluded from the final data analysis due to excessive eye movement artifacts.

\subsubsection{Materials}

The materials comprised 40 sentences for each of the critical conditions (expected versus unexpected) illustrated in example (1) above. Critical verbs were identical across conditions, since the sentential context varied. The 40 sentences per condition were constructed using 10 verbs, thus resulting in 4 repetitions of each individual verb per condition; crucially, sentence contexts differed across repetitions. The verbs were frequent spatial verbs (like STAND, 
SIT, JUMP or LAY) and were checked for grammatical and semantic correctness, frequency and possible dialectical variation with two deaf native informants. We used spatial classifier verbs for two reasons: Firstly, spatial verbs, like many other signs, are signed in neutral signing space and have a holdmovement-hold structure. Therefore, the movement within the transition phase towards the target location of the initial hold ends when the target location is reached. By that, transition phase and sign onset clearly mark two different phases, opposed to a sign without initial hold, in which movement in transition phase and sign movement can merge. Secondly, the handshape of spatial verbs classifies the subject according to certain semantic characteristics of the entities the classified noun refers to. Thus, the verb STAND, for example, has a V-handshape ${ }^{7}$ for humans (indicating the two legs), while it has a B-handshape for flat objects like books or laptops. Therefore, the change of the phonological parameters already transports semantic information, which is accessible within the transition phase. By using spatial classifier verbs as critical items we thus extended the duration of and maximized the semantic information within the transition phase in addition to ensuring that our critical signs all belonged to a single homogeneous class of signs. Within the class of spatial classifier verbs, there were two subtypes: action verbs (i.e. JUMP, CLIMB, MOVE) and non-action verbs (i.e. SIT, STAND, LAY). A complete list of materials is provided in Appendix A. As the two verb types differed in duration (see below), ERP analyses were conducted separately for action and non-action verbs.

The 80 critical sentences resulting from this design (20 per condition and verb type) were interspersed with 80 filler sentences and presented in two different pseudo-randomized orders (counterbalanced across participants). The fillers implemented a separate experimental manipulation unrelated to the present design.

7 Some handshapes used in manual signs match with the handshapes used for letters of the alphabet, and are thus named after them. 
The material was discussed, developed and recorded on video together with two deaf DGS informants (one male, one female). Sentences were recorded with a HDR-XR 550E full-HD camera (25 frames / second) and cut and processed with the video editing software application Adobe Premiere Pro. Each video started with $2000 \mathrm{~ms}$ in which the signer remained motionless, before he/she started to sign the sentence in a natural manner. After the end of the sentence, the signer remained on screen (again motionless) for a further $1500 \mathrm{~ms}$. In total, videos had a length of 8 to 10 seconds. Since sign languages not only use hands and arms for articulation but also head, face, eyes and upper body (see Section 1.1.2), these nonmanual components also appear during signing. To minimize the degree of nonmanual variation, we asked the informants to keep their nonmanual action to a minimum within the possibilities of natural signing. We did not want to exclude nonmanuals entirely, as this would have compromised the naturalness of the stimuli. Furthermore, in order to ensure maximal naturalness, videos were not modified in any way (i.e. there was no cross-splicing, no length adaptation etc.). Figure 1.3 and Figure 1.4 provide video stills of the sign onset (left) and the sign offset (right) of the non-action verb SIT (female signer) and the action verb JUMP (male signer).
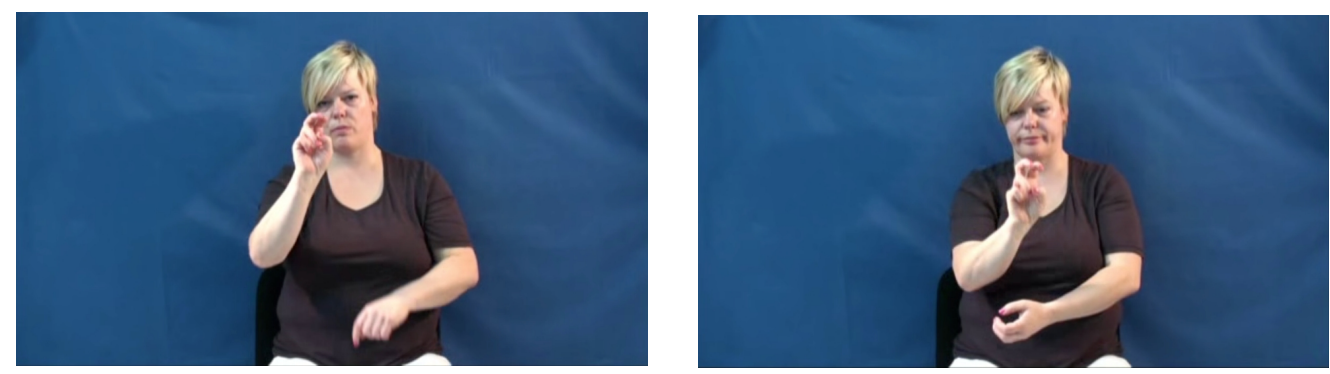

Figure 1.3 Video stills of the sign onset (left) and the sign offset (right) of the non-action verb SIT. 

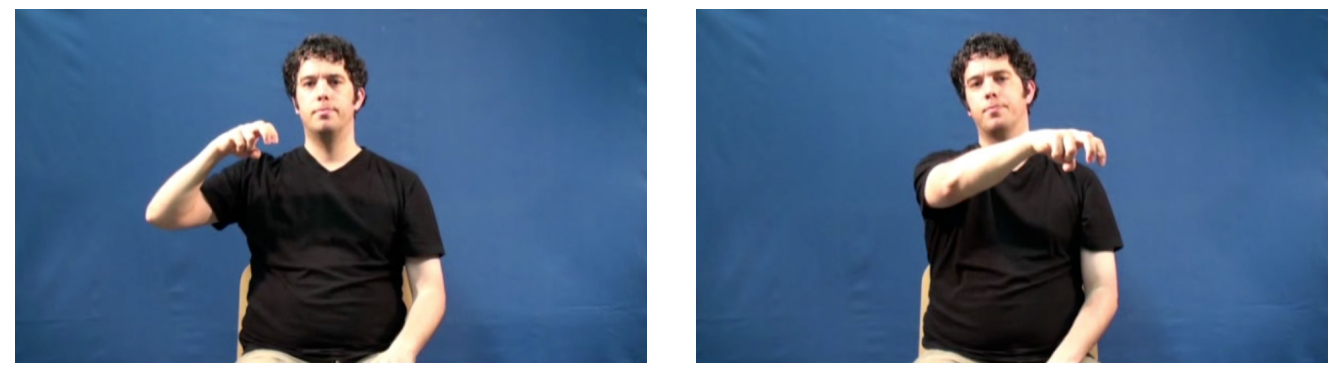

Figure 1.4 Video stills of the sign onset (left) and the sign offset (right) of the action verb JUMP.

An additional set of 10 sentences with the same structure and a final verb that fulfilled the contextual expectation were recorded in the same way as the critical stimuli and presented twice as an exercise before the actual experiment started. In this way, participants were familiarized with the procedure of the trials.

\subsubsection{Procedure}

Experimental sessions were conducted in a dimly lit cabin. Participants were seated in a comfortable chair placed approximately $1 \mathrm{~m}$ in front of a 17 inch computer screen. Each trial began with a sentence-video followed by two tasks: an acceptability judgment (Is the sentence correct or incorrect? - two possible answers: yes, no) and, following $500 \mathrm{~ms}$ of blank screen, an evaluation judgment (How sure are you? - four possible answers: very sure, sure, not sure, not sure at all). Participants were cued to perform the acceptability judgment by the appearance of a question mark (white Arial font (size 60) on a black screen); the evaluation question was cued by a short question (how sure?) in same font. Maximal reaction times for the two tasks were 2000 and $3000 \mathrm{~ms}$, respectively. Following an inter-trial interval of $2500 \mathrm{~ms}$, the presentation of the next video began. Participants were asked to avoid body movement and eye blinks during video presentation. 
At the beginning of the experimental session, all participants saw two introductory videos. The first video was a collection of signed nouns (presented by the same two informants) that could vary within dialects of DGS and that appeared in the critical stimuli. The second video was the introductory video for the experimental session and explained the upcoming procedure. Both videos were presented in DGS, questions were answered if anything was unclear. All communication between the experimenter and the participants took place in DGS.

The experimental session began with a short practice session, followed by 4 blocks of 40 sentences each. Between blocks, participants took short breaks.

\subsubsection{EEG recording}

EEG data were recorded by means of 32 active electrodes placed according to the international 10-20 system (ActiCAP 32, Easycap GmbH, Herrsching, Germany) amplified using a BrainAmp amplifier (Brain Products $\mathrm{GmbH}$, Gilching, Germany) using a sampling rate of $500 \mathrm{~Hz}$. EEG recordings were referenced to the right mastoid online, and re-referenced to linked mastoids. $\mathrm{AFz}$ served as the ground electrode. Average impedances were kept below $10 \mathrm{k} \Omega$. The electrooculogram (EOG) was monitored for each participant with electrodes at the outer canthi of each eye (horizontal EOG) and above and below the right eye (vertical EOG).

\subsubsection{EEG data preprocessing and statistical analysis}

The raw EEG was preprocessed using a $0.3-20 \mathrm{~Hz}$ band-pass filter, which served to eliminate slow signal drifts. Single subject ERP averages were calculated per condition and electrode from -200 to $1500 \mathrm{~ms}$ relative to three different time-locking points (see below). Subsequently, grand averages were computed over all participants. Trials containing EOG or other artifacts were 
excluded from the averaging procedure (the EOG artifact rejection threshold was $40 \mu \mathrm{V}$ level).

To allow for an analysis of the EEG signal in relation to different points in time during the transition phase, we determined three different time-locking (trigger) positions for each video:

(a) Handshape change (hsc). This trigger was placed at the frame during the handshape change in which the hand was tensionless and neither the handshape of the previous sign nor the handshape of the target sign were identifiable.

(b) Target handshape (ths). This trigger point was defined as the first frame in which the target handshape was completely accessible, regardless of target orientation. On average, the target handshape trigger occurred $128 \mathrm{~ms}$ after the handshape change trigger.

(c) Sign onset (on). Sign onset was defined as the first frame of the initial hold for the critical verb, when the target hand configuration reached the target location. On average, sign onset occurred $185 \mathrm{~ms}$ after the target handshape trigger. Average durations from sign onset to sign offset were $427 \mathrm{~ms}$ for non-action verbs and $818 \mathrm{~ms}$ for action verbs.

For each video, each trigger point was identified independently by two linguists with a high expertise in DGS. Conflicting classifications never differed by more than a single frame $(40 \mathrm{~ms})$ and were resolved by discussion. Figure 1.5 illustrates trigger positioning for each of the two critical conditions in one sample video. Note that, since the critical verbs were identical across conditions, we were able to trigger the same frames for each verb (though in different recordings, as these varied depending on the sentence frame).

For the statistical analysis of the ERP data, repeated measures analyses of variance (ANOVAs) were calculated with the factor CONDITION (expected versus unexpected) and topographical region of interest (ROI). Analyses were conducted using R (Team, 2012) and the ez package (Lawrence, 2012). 

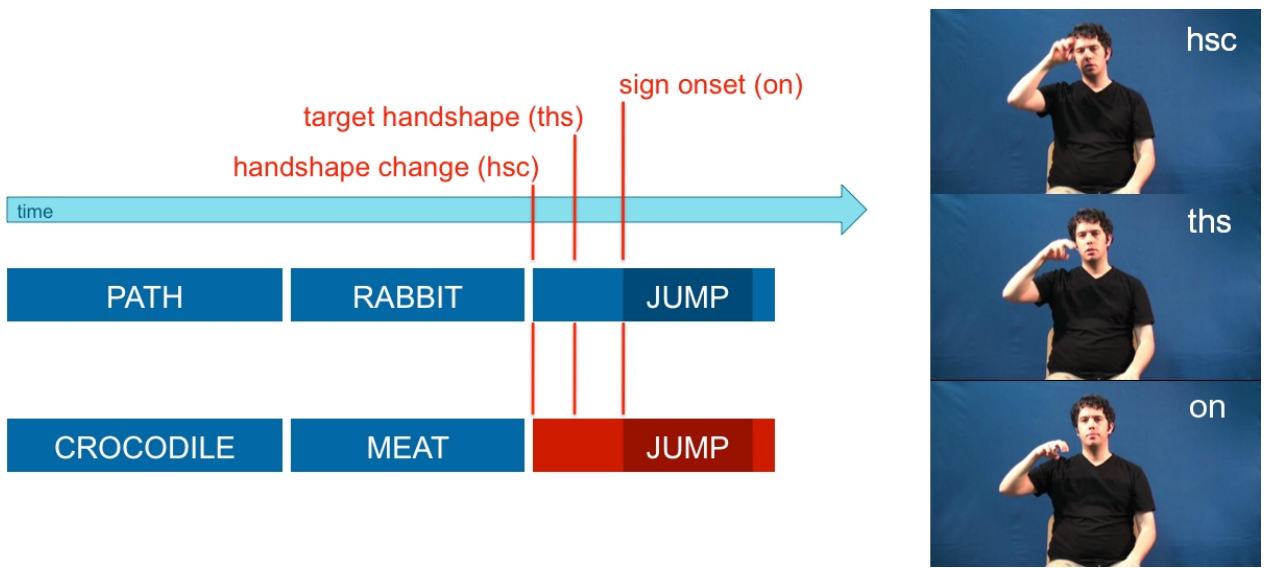

Figure 1.5 Schematic illustration of the trigger positions for ERP time-locking within the transition phase, as well as corresponding video stills for each trigger.

Lateral ROIs were defined as follows: left-anterior (F3, F7, FC1, FC5, C3), right-anterior (F4, F8, FC2, FC6, C4), left-posterior (CP1, CP5, P3, P7, 01), and right-posterior (CP2, CP6, P4, P8, 02). For the analysis of the midline electrodes, FZ, CZ, CPZ, PZ, OZ were each treated as individual ROIs. The statistical analysis was conducted in successive $50 \mathrm{~ms}$ time windows in order to allow for an objective quantification of effect latencies. Effects reaching significance in at least two successive windows were considered reliable (Gunter, Friederici, \& Schriefers, 2000). The statistical analysis was carried out in a hierarchical manner, i.e. only significant interactions $(p<0.05)$ were resolved. Probability values were Huynh-Feldt corrected when appropriate (Huynh \& Feldt, 1970).

\subsection{Results}

\subsubsection{Behavioural data}

Mean acceptability rates were as follows (standard deviations by participants shown in parentheses): action verb - expected: 76.1\% (12.2\%); non-action verb - expected: 83.9\% (16.0\%); action verb - unexpected: 51.4\% (24.2\%); non-action verb - unexpected: 43.4\% (26.3\%). A repeated measures ANOVA 
including the factors CONDITION and VERB-TYPE revealed a significant main effect of CONDITION $\left(F(1,17)=21.86, p<0.001, \eta_{g}{ }^{2}=0.40\right)$ and an interaction of CONDITION and VERB-TYPE $\left(F(1,17)=12.00, p<0.01, \eta_{g}^{2}=0.04\right)$. Resolving the interaction by VERB-TYPE showed significant simple effects of CONDITION for both action $\left(F(1,17)=14.31, p<0.01, \eta_{g}{ }^{2}=0.31\right)$ and nonaction verbs $\left(F(1,17)=25.23, p<0.001, \eta_{g}{ }^{2}=0.48\right)$.

Mean reaction times per condition were as follows: action verb - expected: $780.5 \mathrm{~ms}$ (176.7 ms); non-action verb - expected: $711.5 \mathrm{~ms}$ (177.6 ms); action verb - unexpected: $806.3 \mathrm{~ms}$ (197.1 ms); non-action verb - unexpected: $786.1 \mathrm{~ms}$ (190.6 ms). The statistical analysis revealed no significant main effects or interactions. There was, however, a trend towards an effect of VERB-TYPE $\left(F(1,17)=3.44, p=0.08, \eta_{g}{ }^{2}=0.02\right)$.

In summary, the results of the acceptability judgment task showed that participants judged the sentences with a semantically unexpected final verb as less acceptable than their counterparts with a semantically expected verb. This acceptability difference was apparent for both verb types, but more pronounced for the non-action verbs. In addition, there was a trend towards faster reaction times for sentences with non-action verbs irrespective of expectedness, thus supporting the assumption that participants found the acceptability of these sentences somewhat easier to judge.

Overall, participants were very certain in their judgements of the critical sentences. The mean certainty rating on a $1-4$ scale $(1=$ very certain to $4=$ not certain at all) was 1.85 (standard deviation across participants: 0.42 ).

\subsubsection{ERP data}

Figure 1.6 and Figure 1.7 show grand average ERPs for the sentences with non-action and action verbs, respectively. From both figures, it is apparent that semantically unexpected verbs engendered a broadly distributed negativity followed by a late positivity in comparison to semantically expected 
verbs. The late positivity appears somewhat more pronounced for the nonaction verbs. In addition, visual inspection suggests that the timing of the effects is dependent both on the trigger position and on the type of verb.
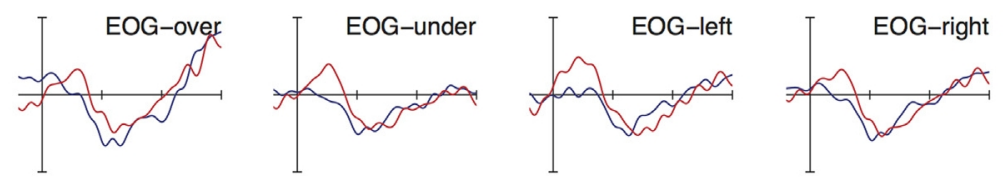<smiles>CCCCCCC(C)CC(C)C</smiles>

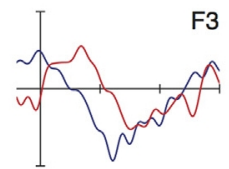

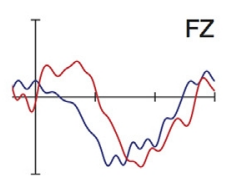

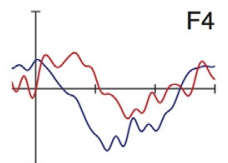

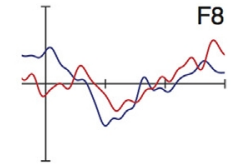

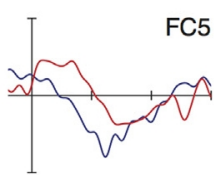

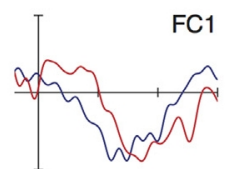

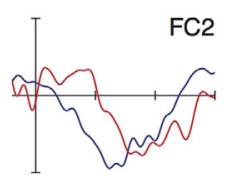

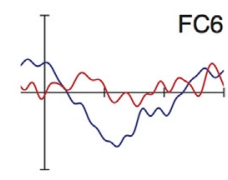<smiles>CC(C)=C1CCCCC1=O</smiles>

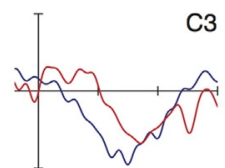

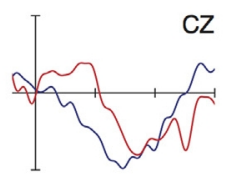

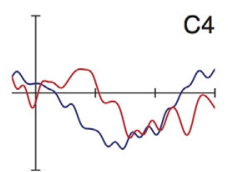

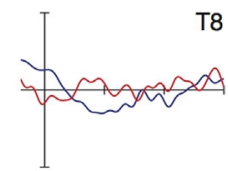

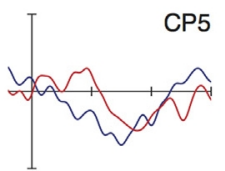

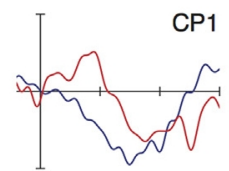

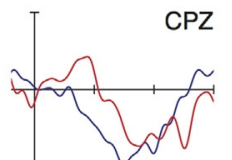

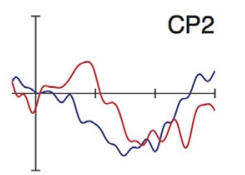

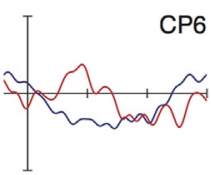
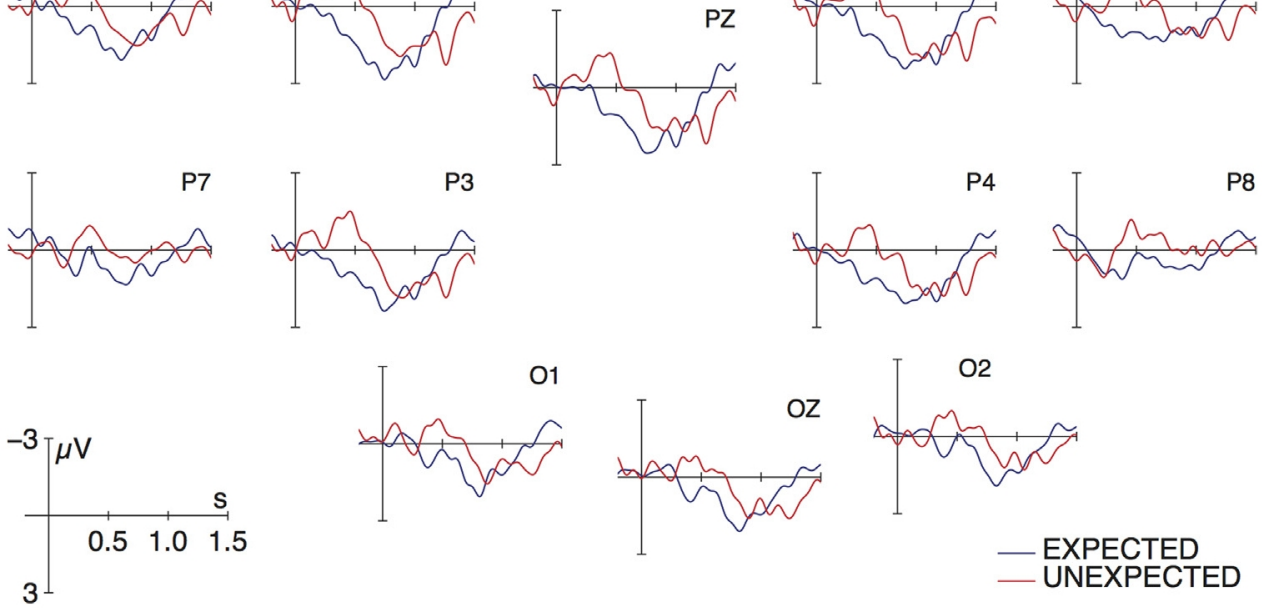

Figure 1.6 Grand average ERPs for unexpected (red line) and expected (blue line) action verbs, time-locked to the handshape trigger. Negativity is plotted upwards. 

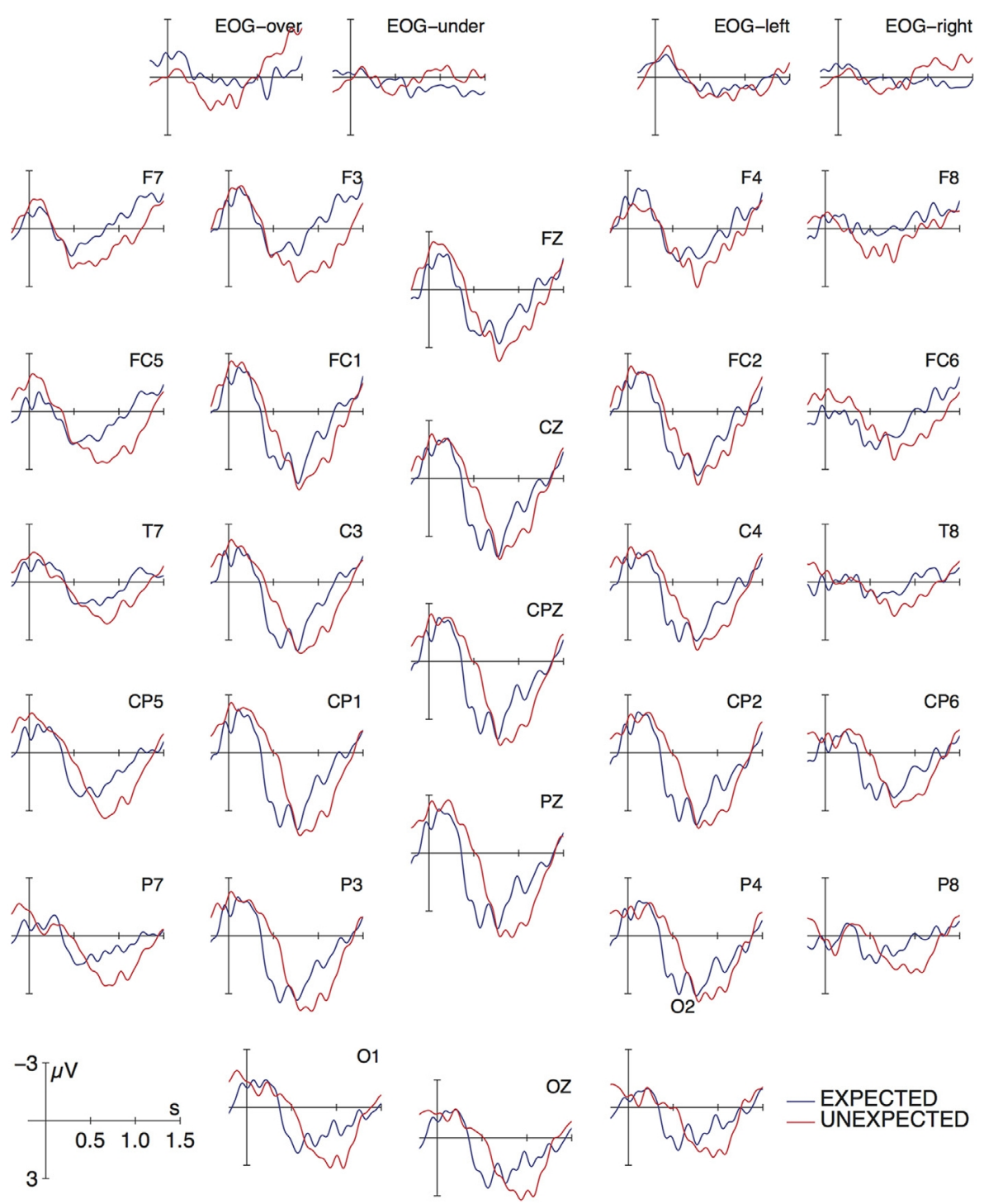

Figure 1.7 Grand average ERPs for unexpected (red line) and expected (blue line) nonaction verbs, time-locked to the target handshape trigger. Negativity is plotted upwards.

These descriptive impressions were confirmed by statistical analyses in successive $50 \mathrm{~ms}$ time windows. The results of the global analyses for each verb type are summarized in Figure 1.8 and Figure 1.9. These revealed, that the negativity effects showed a centro-parietal maximum, thus supporting 
their interpretation as N400 effects. N400 latency, however, depended on trigger position and verb type. For action verbs, N400 effects were observable between 300 and $750 \mathrm{~ms}$ relative to the point of handshape change, between 150 and $500 \mathrm{~ms}$ relative to the onset of target handshape, and between 0 and $350 \mathrm{~ms}$ relative to sign onset. For non-action verbs, N400 effects reached significance in somewhat later time windows and showed shorter durations: they were observable between 500 and $700 \mathrm{~ms}$ relative to the point of handshape change, between 400 and $600 \mathrm{~ms}$ relative to the onset of target handshape, and between 100 and $450 \mathrm{~ms}$ relative to sign onset.

The late positivity only reached significance between 1200 and $1300 \mathrm{~ms}$ (relative to handshape change) and between 1100 and $1200 \mathrm{~ms}$ (relative to the onset of the target handshape) for the action verbs. For the non-action verbs, the positivity effects showed an earlier onset and a considerably longer duration, reaching significance between 950 and $1350 \mathrm{~ms}$ relative to the position of handshape change, between 800 and $1250 \mathrm{~ms}$ relative to the onset of the target handshape, and between 950 and $1150 \mathrm{~ms}$ relative to sign onset.

In summary, semantically unexpected versus expected clause-final verbs engendered N400 effects that were clearly time-locked to events prior to sign onset, as evidenced by $\mathrm{N} 400$ onsets of $0 \mathrm{~ms}$ or $100 \mathrm{~ms}$ (for action and nonaction verbs, respectively) relative to sign onset. By contrast, ERPs timelocked to the earlier events of handshape change and target handshape onset showed much more typical N400 onset latencies. In addition, N400 latency was modulated by verb type: action verbs showed an earlier onset of the negativity effect across all critical trigger points, with differences ranging in magnitude from $100 \mathrm{~ms}$ (sign onset) to $250 \mathrm{~ms}$ (target handshape). Finally, we also observed late positivity effects for unexpected versus expected continuations with both verb types. These showed a more consistent latency across trigger points than the $\mathrm{N} 400$ effects and, interestingly, an earlier onset for non-action verbs (from $800 \mathrm{~ms}$ relative to target handshape onset) than action verbs (from $1100 \mathrm{~ms}$ relative to target handshape onset). 
LATERAL ELECTRODE SITES

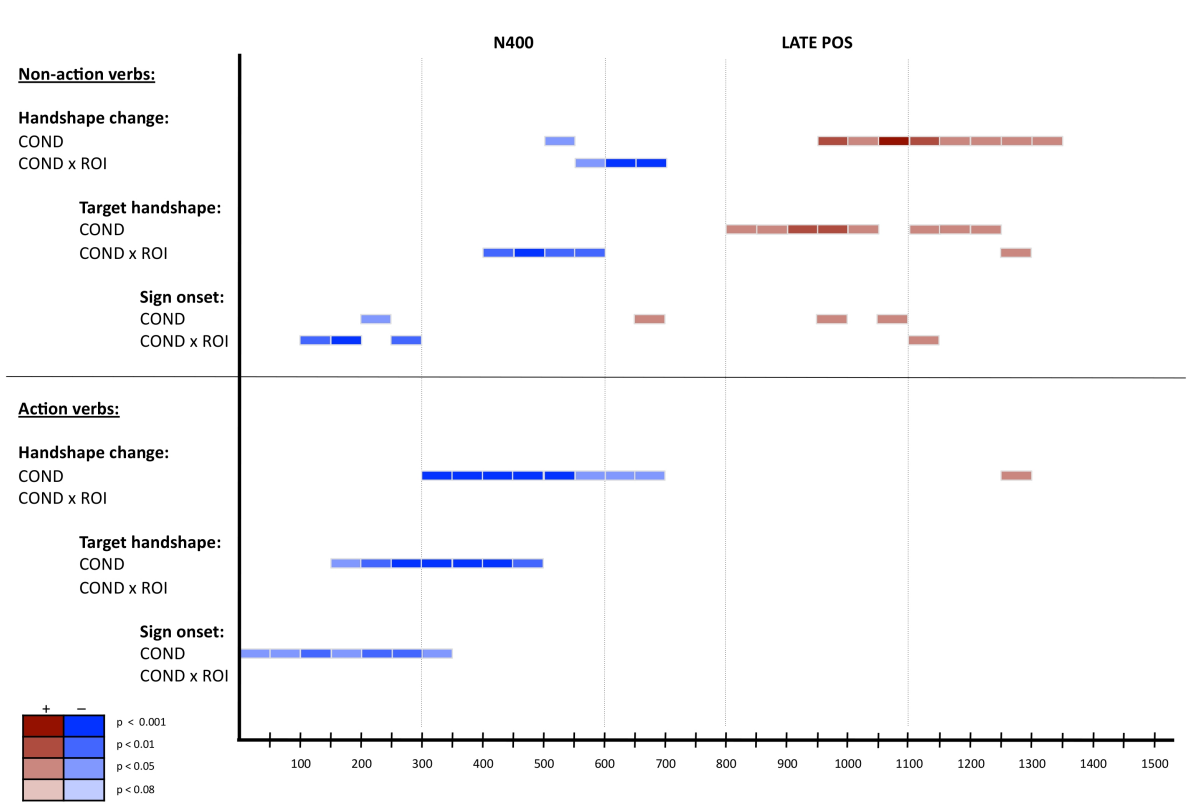

Figure 1.8 Summary of the statistical analyses in successive $50 \mathrm{~ms}$ time windows for the lateral electrodes.

MIDLINE ELECTRODE SITES

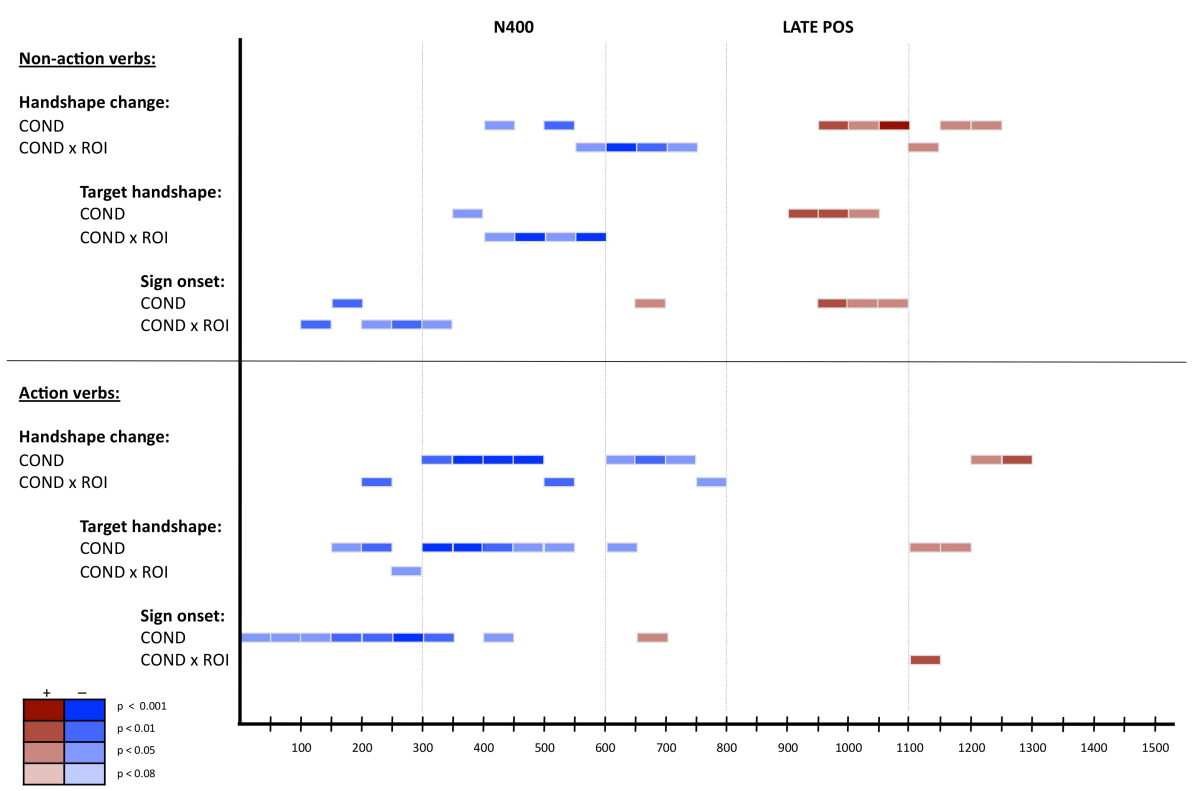

Figure 1.9 Summary of the statistical analyses in successive $50 \mathrm{~ms}$ time windows for the midline electrodes. 


\subsection{Discussion}

The present ERP data from German Sign Language (DGS) demonstrate that predictions with respect to an upcoming clause-final sign can already be falsified in the transition phase leading up to that sign, i.e. prior to critical sign onset. This is evidenced by N400 effects timelocked to events preceding sign onset, namely the point of change of the signer's handshape and onset of the target handshape. Furthermore, as N400 effects are remarkably invariant with regard to onset latency (Federmeier \& Laszlo, 2009), it appears legitimate to use the different onset latencies observed across our three trigger positions to infer which position was informative enough to induce a lexical prediction error. In this regard, we propose that handshape change constituted the critical time-point for the action verbs (N400 onset latency: $300 \mathrm{~ms}$ ), while the $\mathrm{N} 400$ effect for the non-action verbs was most likely elicited by the onset of the target handshape (N400 onset latency: $400 \mathrm{~ms}$ ).

These results provide converging support for the hypothesis that prediction during language comprehension is subserved by forward models. Our data show that, in addition to allowing for an anticipation of the critical sign itself, prediction must be specific enough in order for a prediction error to occur during the transition phase leading up to that sign. Thus, the mechanisms of prediction must go beyond the mere preactivation of amodal lexical entries and rather provide very specific, modality-dependent information regarding predicted upcoming words or signs. Forwards models can supply precisely this type of information: Anticipation of a particular concept leads to the prediction of a modality-specific form in which that concept will be expressed and, thereby, to an anticipated trajectory linking the appropriate sign with the previous sign. When the signing trajectory actually encountered mismatches with the anticipated trajectory (i.e. the output of the forward model), an N400 effect is elicited. In this way, our results from sign language processing are highly compatible with the finding of early negativities in response to phonological mismatches with a semantically expected word (i.e. the finding that negativity effects are elicited earlier when the initial phonemes of a 
critical word are incompatible with the predicted word as opposed to when the initial phonemes match those of the predicted word; Connolly \& Phillips, 1994; van den Brink, Brown, \& Hagoort, 2001). Assuming prediction via a forward model, the findings from the auditory domain and those from sign language both reflect a modality-specific prediction error. In contrast to the results from auditory processing, however, our data from sign language provide evidence that this type of prediction error can even be induced prior to critical word/sign onset. An account along these lines is highly compatible with proposals positing that prediction depends on the language production system (Federmeier, 2007; Pickering \& Garrod, 2007), though this was not tested specifically in the present study.

In addition to providing new evidence regarding the application of forward models during language comprehension, our results call for a new interpretation of transition phases in sign language. It is apparent from our data that the transition phase cannot be treated as a "meaningless" trajectory that serves to link two meaningful signs with one another, but that it rather carries a substantial amount of meaning itself. It must be stressed, however, that the present study only demonstrates that this information can induce a prediction error. It does not, conversely, show that the degree of information suffices to allow for sign recognition. Whether or not this is the case is an interesting question for future research. Based on the present findings and the assumption of a forward model, we would predict that the transition phase should allow for sign recognition at least under certain circumstances, namely when the combination of sentence context and trajectory provides enough information for recognition of the upcoming sign.

The wealth of information provided by the transition phase is further underscored by the observation of different critical onset points for the prediction error responses for action and non-action verbs. Though these differences were not predicted prior to the present study, they can possibly be derived by differing characteristics of the transition phase for each verb type. Non-action verbs differ from action verbs in that their path movement 
indicates that "the referent is located here" instead of that "the referent moves from here to there". Supalla (1982) calls this be-located movement the "contact morpheme" because the referent has to be in contact with something in order to be located. Therefore, the path movement of non-action verbs is not informative with regard to the path of motion, nor with regard to a certain manner of motion (because the referent itself is not moving). Furthermore, we observed additional differences in the trajectories of nonaction and action verbs. Trajectories of non-action verbs typically included a slight upward movement towards the i-hold of the target sign (i.e. towards the be-located or "contact" morpheme). This upward movement was not observed in the trajectories of action verbs. Thus, the secondary transition in the form of the short upward movement - to the onset of the be located movement, may be responsible for the delay of the N400 onset. ${ }^{8}$ Since it is common to all non-action verbs, it neutralizes further semantic differences for a short period of time. For action verbs, by contrast, the trajectory of the hand movement is more strongly predictive of verb meaning from its onset. However, whether these differences between the trajectories are responsible for the latency shift in the N400 effect needs further empirical investigation.

In addition to N400 effects, the semantically unexpected versus expected continuations elicited late positivity effects in the present study. This result is not surprising, as semantically anomalous sentences have been shown to engender positivity effects in addition to N400 effects in a number of previous studies employing acceptability judgment tasks (e.g. Roehm, BornkesselSchlesewsky, Rösler, \& Schlesewsky, 2007; Sanford, Leuthold, Bohan, \& Sanford, 2011). Interestingly, however, the late positivity effects observed here showed a greater invariance of onset latency with regard to the different trigger points than the N400 effects. Moreover, they showed a reversed pattern of onset latency to the $\mathrm{N} 400$ with respect to the two verb types examined: Late positivity onset occurred earlier for non-action verbs than for action verbs, while N400 effects showed the inverse pattern. Taken together,

${ }^{8}$ We would like to thank an anonymous reviewer for this suggestion. 
these observations suggest that positivity onset was not determined by the same critical event as N400 onset. If this were the case, both types of effects should be expected to show the same overall pattern of onset latency variation, with the positivity simply time-shifted with respect to the $\mathrm{N} 400$ by some constant. Rather, we propose that, while N400 onset was determined by the point at which a prediction error with respect to the upcoming sign could be recognized (see above), positivity onset was more closely time-locked to participants' responses and, hence, to critical sign offset rather than onset or the pre-onset trigger points (recall that the action verbs had a longer duration than the non-action verbs). This assumption is in line with a recent study which used single-trial analyses to decompose an N400 - late positivity scalp ERP pattern engendered by linguistic anomalies versus control sentences (Sassenhagen, Schlesewsky, \& Bornkessel-Schlesewsky, 2014). Results revealed that, while N400 effects were time-locked to critical stimulus onset, positivity effects were response-locked. Sassenhagen and colleagues interpret this result as evidence for the proposal that late positivities in language processing should be viewed as members of the domain-general P300 family (e.g. Coulsen, King, \& Kutas, 1998; Kretzschmar, 2010; Roehm et al., 2007) and link this idea to a recent neurophysiological model of the P300, the Locus Coeruleus-Norepinephrine (LC/NE-P3) model (Nieuwenhuis, Aston-Jones, \& Cohen, 2005). According to this model, the P300 results from activation of the Locus Coeruleus (i.e. the brain stem source for noradrenergic projections to the cortex) following the detection of subjectively significant events. This results in a release of norepinephrine, thereby increasing neural responsivity to a particular stimulus and influencing the behavioral response to it. From this perspective, the late positivity in the present study reflects the behaviorally relevant categorization of a stimulus as unacceptable (see also Bornkessel-Schlesewsky et al., 2011)) rather than linguistic processing per se. This proposal is further compatible with the observation that the positivity effects were more pronounced for the sentences with non-action verbs, since participants showed a clearer behavioral discriminability between expected and unexpected continuations for this verb class. 


\subsection{Summary and conclusions}

The present ERP study on German Sign Language demonstrated that prediction mismatches with an unexpected sign can engender N400 effects even before sign onset, i.e. via properties of the transition phase between the preceding sign and the critical sign. This finding suggests that predictions about upcoming material within the linguistic input stream go beyond the preactivation of amodal signs and rather provide very detailed modalityspecific information about the anticipated realization of the predicted item. We have argued that this observation is highly compatible with the use of production-based forward models in language comprehension. 


\title{
2 Signs activate their written word translation. An ERP study on cross-modal co-activation in German Sign Language
}

\begin{abstract}
Language co-activation between a native sign language (L1) on the one hand and of a secondly acquired spoken language (L2) on the other hand in deaf bimodal bilinguals is still mysterious. In the present study, we investigated two priming conditions with deaf native bilinguals of German Sign Language (DGS) and German. Participants saw signed sentences in DGS that either contained a prime and a target sign with overt phonological overlap as signs, i.e. phonological priming in DGS, or a prime and target sign which were phonologically unrelated as signs but had a covert orthographic overlap in their German translation, i.e. orthographic priming in German. An event-related potential measurement showed a significant priming effect for both conditions. Target signs that were either phonologically related as signs or had an underlying orthographic overlap in their German translation engendered a reduced negative polarity in the electrophysiological signal compared to overall unrelated control targets. We thus provide first evidence that deaf native bilinguals activate their secondly acquired spoken language during whole sentence processing of their native sign language. For an explanation of this link between a sign and the corresponding spoken word representation, we discuss the nonmanual component mouthing as a shared phonological representation element for signs as well as for spoken words.
\end{abstract}

\subsection{Introduction}

Congenitally and profoundly deaf people make up an exceptional and specific group of bilinguals. Next to the acquisition of a native sign language, they must also overcome the challenge of learning a spoken language with no or limited phonological input. Nonetheless, they can achieve a high proficiency in their second (spoken) language by using other cues from second language experience, e.g., visual mouth representations of spoken words and orthographic representations of written words (cf. Plaza-Pust \& Weinmeister, 2008). Therefore, in the last few years, bimodal bilingual research has 
focused much attention on how the two languages of different modalities interact and co-activate one another without phonological form-based similarity (cf. Shook \& Marian, 2010).

It is widely acknowledged that the two spoken languages of unimodal bilinguals influence the processing of one another (Dijkstra \& Heuven, 2002; Kroll \& de Groot, 2005; Marian, Spivey, \& Hirsch, 2003; Thierry \& Wu, 2007). Additionally, recent studies with bimodal bilinguals (i.e., bilinguals who are highly proficient in a sign and a spoken language) reveal a similar influence of participants' sign language knowledge on spoken language production (Casey \& Emmorey, 2008; Emmorey, Borinstein, Helsa, Thompson, \& Gollan, 2008; Pyers \& Emmorey, 2008) and on written language processing (Kubus, Villwock, Morford, \& Rathmann, 2014; Morford, Wilkinson, Villwock, Piñar, \& Kroll, 2011; Ormel, Hermans, Knoors, \& Verhoeven, 2012; Shook \& Marian, 2012). This type of investigating cross-modal cross-language activation in bimodal bilinguals allows a great opportunity to examine the domain-general mechanisms underlying language processing. Particularly, because lexical items of the two different languages involved are perceived and produced in contrary modalities (auditory-articulatory vs. visual-manual), and thus do not share phonological form-based similarities.

In contrast to previous studies on bimodal bilingualism that focused on the co-activation of the native sign language (L1) when processing isolated words in the second (written) language (L2) (Kubus et al., 2014; Morford et al., 2011; Ormel et al., 2012; Shook \& Marian, 2012), we present evidence for coactivation in the opposite direction: We examined the extent to which target signs integrated in sentences in German Sign Language (DGS, i.e., L1) coactivate orthographic (and phonological) forms of the German translation equivalents (i.e., L2).

Fifteen congenitally deaf bilinguals of DGS (native L1) and German (early L2) participated in this ERP experiment with two priming conditions: In the first condition - within language priming - a prime and a target sign were presented in a DGS sentence, overlapping in three out of four sign phonological 
parameters (e.g., the two DGS signs STORE - ANIMAL only differ in the parameter 'movement'). In the second condition - cross language priming prime and target were again presented in a DGS sentence and were phonologically unrelated to one another. However, the German translation equivalents of these signs overlapped orthographically and phonologically (e.g., Mutter - Butter, 'mother' - 'butter'). Thus, we examined the possibility of cross-modal cross-language co-activation of L2 representations during L1 sign language processing. We recorded participants Electroencephalograms and evaluated event-related potentials (ERPs) relative to the onset of target signs (in within language priming condition, in cross-language priming condition, and, respectively, in control conditions).

There are, however, no other ERP studies investigating cross-language priming effects in sign languages; and importantly for the purposes of the current study, no ERP studies examining cross-modal cross-language activation of L2 orthographic translation equivalents during sign language processing. This experimental design of overt and covert phonological priming during whole sentence processing is based upon previous studies on phonological priming effects in sign language processing. In the following section we review overt phonological priming studies in sign languages (Section 2.1.1), before introducing the current state of art in matters of general cross-language activation studies (Section 2.1.2) and highlight most recent investigations of cross-language co-activation in bimodal bilinguals (Section 2.1.3). After a presentation of the design of the current study (Section 2.1.4), the methodological procedure (Section 2.2) and our results (Section 2.3), we will discuss the outcomes with respect to current theoretical explanations of the operative basics of cross-modal cross-language activation (Section 2.4). Section 2.5 summarizes the study and concludes with final remarks.

\subsubsection{Phonological priming in sign languages}

Studies on sign language phonology reveal a remarkable structural overlap between spoken language phonology and sign language phonology, with 
respect to sub-lexical phonological units and neural networks (Brentari, 2012; MacSweeney, Waters, Brammer, Woll, \& Goswami, 2008; Meier, Cormier, \& Quinto-Pozos, 2002; Sandler \& Lillo-Martin, 2006). For example, manual signs are constituted by four basic phonological parameters: the handshape, the orientation of the palm, the location in signing space, and the path movement (first identified by Stokoe, 1960 and Stokoe et al., 1965), each describing a group of phonological features (cf. Brentari, 2012). Thus, studies examining phonological priming in sign language find similar effects to studies examining phonological priming in spoken language. They typically present deaf native signers with a prime and a target sign, where the prime and target sign overlap in one, two or three out of the four phonological parameters. They find in facilitation or inhibition effects (i.e. faster or slower reaction times of a decision task relative to the target, depending on the overlap with the prime) that recognition of the target is influenced by the degree of overlap between prime and target signs (Corina \& Hildebrandt, 2002; Dye \& Shih, 2006; Gutiérrez et al., 2012a; Orfanidou et al., 2009). Thus, the processing of the phonological features of a prime sign has an impact on the processing of a subsequently appearing target sign.

Since phonological features of manual signs appear simultaneously rather then sequentially (a certain handshape appears at a certain location with a certain hand orientation), sign language priming studies have primarily focused on the impact each phonological parameter has on sign processing. The path movement of a sign is the most salient phonological parameter (and can be compared to the syllable peak of spoken words, Brentari, 1990) and is realized after the handshape and the location have been formed. Hence, priming studies so far laid a predominant interest in examining priming effects of handshape overlap versus location overlap. The outcomes of these studies are quite heterogeneous, but reveal a general difference in the processing of handshape versus the processing of location. While priming effects on handshape priming are rather rare (e.g., Carreiras et al., 2008 only found an facilitation effect for non-signs), most studies report an inhibitory priming effect for location priming, i.e., a delayed recognition of the target 
when primed by a sign overlapping in location (Corina \& Hildebrandt, 2002; Carreiras et al., 2008). In contrast, Dye \& Shih (2006) found a facilitation priming effect for sign pairs that overlapped in location and movement (i.e., targets were identified faster when they shared location and movement with the prime). They thus conclude that the combination of location and movement of a sign is decisive during the initial phase of its lexical access.

Because each phonological parameter might have a different impact on sign processing, a crucial aspect of such priming studies is the identification of "phonologically similar signs". While most studies identify sign similarity by an overlap of either two (Morford et al., 2011) or three (Shook \& Marian, 2012) phonological parameters, some investigated phonological overlap of only one parameter (e.g., Corina \& Hildebrandt, 2002; Carreiras et al., 2008; Gutiérrez et al., 2012a). A further issue for generating prime-target pairs in priming studies is the fact that manual signs can be relatively ambiguous. For example, as most of the sign languages investigated so far, DGS has a very limited sequential affixal morphology. Therefore, there is no overt morphological marker for nouns, verbs or adjectives, which makes some signs ambiguous with respect to word classes. For instance, the DGS sign WORK can be used nominally as well as verbally, as can be seen in (2) (Schwager \& Zeshan, 2008: p. 533-534, see also Meir, 2012).

(2) a. WORK FIND DIFFICULT\#INTS [original example 26]

'It is very difficult to find a job.'

b. POSS 1 WIFE WORK GOOD [original example 20] 'My wife works well.'

In addition, many signs in DGS are homonyms, like e.g., TECHNOLOGY and POLITICS. Both signs are produced with the identical handshape, orientation, location and movement. Homonym signs can be distinguished in DGS by an accompanying nonmanual component called 'mouthing'. This refers to the silent pronunciation of (parts of) the spoken word equivalent simultaneous to 
the production of the manual sign, here the German syllables "tech" or "poli". The linguistic status of mouthing is still under debate within the sign language research community, either being categorized as a loan element from the surrounding spoken language, or being seen as an integral part of the sign language grammar (cf. Braem \& Sutton-Spence, 2001). Hence, priming studies in sign languages are confronted with a high ambiguity between phonological identical signs in matters of word classes on the one hand and semantically different homonyms on the other hand. Nevertheless, all priming studies indicate that signs can form minimal pairs with other signs differing in only one phonological parameter, and that successive presentation of minimal pair signs can influence the processing of one another.

While there have been few ERP studies on sign language priming, the study by Gutiérrez et al. (2012a) is a notable exception examining form-based priming in Spanish Sign Language (LSE). They investigated the influence of handshape versus location overlap between prime and target signs on the neural correlates of target sign processing. Gutiérrez et al. (2012a) find more negative deflections in brain activity to target signs primed by location overlapping primes only. Compared to the processing of unrelated primes, this reflects greater difficulty in target processing in the overlapping condition relative to the unrelated condition. While one might have expected greater ease in target processing in the overlapping condition, we note that Gutiérrez and colleagues examined single parameter overlap between prime and target signs, i.e., signs overlapped in either handshape or location only. They explain this effect based on interactive activation models of processing (e.g., McClelland \& Elman, 1986; McClelland \& Rumelhart, 1981; Norris, 1994), which highlight inhibitory connections between form-overlapping words at the lexical level. Indeed, similar effects are reported in the spoken language literature, which find inhibitory priming effects in tasks where primes and targets differ in just the onset phoneme and facilitatory priming effects in tasks where primes and targets overlap in more than one phoneme, e.g., rhyme-priming effects (Mani, Durrant \& Floccia, 2012; Slowiaczek et al., 1987; Emmorey, 1989; Burton, 1992; Corina, 1992; Radeau et al., 1995). 
There are, however, no ERP studies investigating cross-modal cross-language activation with bimodal bilinguals.

\subsubsection{Cross-language co-activation}

Bilingual research has long focused on examining the degree to which one language is activated while processing input of another language, typically called cross-language co-activation. Of interest here is the identification of the factors that facilitate or inhibit such co-activation, and the description of the cognitive links between different levels of representation in the two languages. We, therefore, distinguish three groups of bilinguals: first, hearing unimodal bilinguals, who are proficient in two spoken languages, second, hearing bimodal bilinguals, typically children of deaf adults (i.e. CODAs), who are proficient in a sign and a spoken language, and third, deaf bimodal bilinguals, who are also proficient in a sign and a spoken language

Most research on unimodal (hearing) bilinguals examines the impact of phonological and/or orthographic similarities between two spoken languages on cross-language co-activation. This work finds that processing L1 or L2 input routinely involves co-activation of phonologically and/or orthographically related words from the other language (Bijeljac-Babic, Biardeau, \& Grainger, 1997; de Groot, Delmaar, \& Lupker, 2000; Heuven, Dijkstra, \& Grainger, 1998; Lemhöfer, Dijkstra, \& Michel, 2004; Van Wijnendaele \& Brysbaert, 2002). While most studies have examined this issue using an explicit form-based overlap across the two spoken languages - i.e., by presenting L1 and L2 translation equivalents that overlap phonologically and/or orthographically - Thierry \& Wu (2007), however, present an implicit priming paradigm. In an ERP study with a semantic relation judgment task, Chinese-English bilinguals saw English word pairs whose Chinese translations either overlapped orthographically (e.g. post [you zheng] - mail [you jian]) or did not. Word pairs whose translation overlapped in Chinese showed a less negative N400 amplitude compared to word pairs unrelated in 
Chinese, suggesting that the processing of English words co-activated the Chinese translations of these words. Their findings demonstrate unconscious co-activation of a native language (Chinese) during second language processing (English). Further studies with unimodal bilinguals replicated the finding of covert cross-language co-activation (Altvater-Mackensen \& Mani, 2011; Blumenfeld \& Marian, 2007; Canseco-Gonzales et al., 2010; Wu \& Thierry, 2010).

Correspondingly, research on bimodal bilinguals also suggests a substantial influence of one language modality during the production and/or perception of the other language modality. First production studies with hearing bimodal bilinguals (i.e., CODAs) have examined cross-language influences of the grammatical structure of the sign language on the grammar of the spoken language. For example, analysis of written sentences in an online email listserv for American CODAs revealed typical ASL structures in the English texts, including subject drop, copula drop, preposition drop, auxiliary drop, etc. (Bishop \& Hicks, 2005). Other evidence for an influence of sign language knowledge on spoken language production comes from co-speech gestures (Casey \& Emmorey, 2008), and facial expressions (Pyers \& Emmorey, 2008). In contrast to unimodal bilinguals, who can frequently switch between their two languages ("code-switching"), bimodal bilinguals have the further ability to produce signs simultaneously to spoken words, so-called "code-blends", which suggests a high level of interconnectivity between both languages (Emmorey et al., 2003, Emmorey et al., 2008, Donati \& Branchini, 2013). Emmorey et al. (2008) introduce a language production model for bimodal bilinguals that explains this connective link. Next to a "Message Generator" that engenders the content of the articulation (either in spoken or in sign language), an "Action Generator" is responsible for creating an action plan. Both interact on a communication planning level, leading to co-speech gestures and code-blends alongside vocal or manual articulation.

Next to research on the cross-modal influences in sign and spoken language production, experimental investigations of cross-modal co-activation during 
language processing has become of greater interest. Over the last years, a number of language processing studies with deaf bimodal bilinguals investigated the co-activation of L1 signs during L2 word processing (Morford et al., 2011; Ormel et al., 2012; Shook \& Marian, 2012; Kubus et al., 2014). All of them found priming effects for word pairs, or word-picture pairs, whose sign translations were phonologically related, suggesting underlying co-activation of the L1 sign representation during L2 word processing. In the subsequent section we review the most recent studies on cross-modal crosslanguage activation.

\subsubsection{Previous cross-language co-activation studies with bimodal bilinguals}

Morford et al. (2011) were the first to examine whether deaf bimodal bilinguals activate signs during single word reading. They adapted Thierry \& Wu's (2007) semantic relation judgment paradigm with English word pairs. 19 deaf American Sign Language (ASL) - English bilinguals judged whether two English words were "related in meaning" or not (Morford et al., 2011: p. 288). Half of the stimuli were semantically related, while the other half were semantically unrelated. Unbeknown to the participants, half of the words (semantically related as well as unrelated word-pairs) were also phonologically related in their ASL translations (phonological relation was defined as sharing a minimum of two phonological parameters). For example, the ASL signs PAPER and MOVIE share the same handshape and location, and differ only in the orientation and in the secondary movement of the dominant hand. Importantly, only written words were presented that shared no orthographic or phonological overlap in English. Any modulation of the priming effect based on phonological ASL overlap must thus be the result of the co-activation of the ASL sign representation upon reading the corresponding written English word. Reaction time measurements showed indeed that participants responded faster to semantically and phonologically related items (+sem/+phon, like ASL BIRD and DUCK) compared to seman- 
tically related, but phonologically unrelated items (+sem/-phon). Accordingly, the rejection of semantically unrelated but phonologically related items (-sem/+phon) was slower compared to overall unrelated items (-sem/-phon). Thus, the processing of the (overt) semantic overlap between written words was modulated by the (covert) phonological ASL overlap between the corresponding signs. Morford et al. (2011), thereby, provide compelling evidence that deaf bilinguals activate sign representations during single (written) word processing. For comparable results of cross-modal crosslanguage activation in German Sign Language with an analogous experimental design, see Kubus et al. (2014).

Similarly, Ormel et al. (2012) conducted an experiment on cross-language coactivation with bimodal bilingual children. In a word-picture verification task, deaf school kids (grade 3-4 and grade 5-6) read a Dutch word and had to decide whether a subsequently presented picture matches the word or not. In the "mismatch" condition (i.e. no match between word and picture), the sign translation of the Dutch word and the sign for the picture were either phonologically related or not. ${ }^{9}$ In contrast to Morford et al. (2011), who found a facilitation effect for underlying phonological relation, Ormel et al. (2012) found an inhibition effect for those word picture pairs, whose sign translations shared phonological overlap: The response time to word-picture pairs whose sign translations phonologically overlapped was slower compared to word-picture pairs with no phonological overlapping sign translations. This is accompanied by the effect that deaf children were less accurate in their decision when it came to the former condition compared to the latter. Therefore, Ormel et al. (2012) suggest that features of the phonology of the native Sign Language of the Netherlands (NGT, L1) were coactivated during processing of words in the second language Dutch (L2). This cross-modal cross-language activation occurs already "before deaf children have attained full proficiency in either language" (Ormel et al., 2012: p. 300).

${ }^{9}$ Items with phonological overlap (like DOG and CHAIR in NGT) were not identified by the number of shared phonological parameters, like in Morford et al. (2011). Rather, Ormel et al. (2012) established phonologically strong related items previously by an independent phonological similarity judgment task. 
Although with different outcomes, Ormel et al. (2012) and Morford et al. (2011) provide evidence for cross-modal cross-language activation in deaf bimodal bilinguals, using post-processing decision-making tasks. In contrast, recent work by Shook \& Marian (2012) provide a more online measure of bimodal co-activation in hearing bimodal bilinguals (CODAs). In an eyetracking study using a visual-world-paradigm, hearing CODAs were asked in spoken English to "click on the ...". Participants then saw images of four items: the named target (e.g., cheese), two unrelated distractors (stamp, watch) and a critical competitor (paper), whose sign translation overlapped phonologically (across three phonological parameters) with the sign translation of the target word. In any other respect, the items had no semantic relation to each other and the English words did not overlap phonologically. Based on the finding that participants looked at competitor items more often and longer than at distractor items, Shook \& Marian (2012) conclude that sign translations of the target word were co-activated during the processing of the target. This coactivation led to some activation of the related competitor sign translation either through phonological overlap or direct lexical co-activation. Thus, hearing bimodal bilinguals also show cross-language co-activation of sign representations during spoken word processing.

All studies so far investigated the co-activation of the native sign language (L1) during single word processing of the second spoken language (L2). Table 2.1 gives an overview of hitherto existing studies of cross-language coactivation of bimodal bilinguals (deaf and hearing). 
Table 2.1 Overview of studies on cross-language activation with bimodal bilinguals.

\begin{tabular}{|c|c|c|c|c|}
\hline $\begin{array}{l}\text { authors; } \\
\text { year }\end{array}$ & $\begin{array}{c}\text { experimental design; } \\
\text { method }\end{array}$ & $\begin{array}{c}\text { participants; } \\
\text { languages }\end{array}$ & $\begin{array}{l}\text { activation; } \\
\text { prod/perc }\end{array}$ & $\begin{array}{c}\text { results } \\
\text { (selection) }\end{array}$ \\
\hline $\begin{array}{l}\text { Hanson } \\
(1982)\end{array}$ & $\begin{array}{l}\text { probe recall of word/ } \\
\text { sign lists }\end{array}$ & \begin{tabular}{|l|} 
deaf \\
bilinguals \\
(ASL-Eng.)
\end{tabular} & $\begin{array}{l}\text { production } \\
\text { of signs }\end{array}$ & $\begin{array}{l}\text { signs with phonological } \\
\text { similar Engl. translations } \\
\text { showed less accuracy in } \\
\text { recall }\end{array}$ \\
\hline $\begin{array}{l}\text { Treiman \& Hirsh- } \\
\text { Pasek (1983) }\end{array}$ & $\begin{array}{l}\text { read written text; } \\
4 \text { exp. on recode } \\
\text { strategy, e.g. into } \\
\text { signing }\end{array}$ & \begin{tabular}{|l|} 
deaf \\
bilinguals \\
(ASL-Eng.)
\end{tabular} & $\begin{array}{l}\mathrm{L} 2 \rightarrow \mathrm{L} 1 ; \\
\text { perception }\end{array}$ & $\begin{array}{l}\text { participants recoded } \\
\text { read text into signing }\end{array}$ \\
\hline $\begin{array}{l}\text { Hanson \& Feldman } \\
(1989)\end{array}$ & $\begin{array}{l}\text { Engl. lexical decision } \\
\text { task (written words) } \\
\text { (RT); prime-target } \\
\text { shared } \\
\text { (a) morph. in Eng+ASL } \\
\text { (b) morph. in ASL } \\
\text { transl. }\end{array}$ & \begin{tabular}{|l} 
deaf \\
bilinguals \\
(ASL-Eng.)
\end{tabular} & $\begin{array}{l}\mathrm{L} 2 \rightarrow \mathrm{L} 1 ; \\
\text { perception }\end{array}$ & $\begin{array}{l}\text { only facilitation effect for } \\
\text { (a), not for (b) }\end{array}$ \\
\hline $\begin{array}{l}\text { Grote \& Linz } \\
\text { (2003) }\end{array}$ & $\begin{array}{l}\text { written word } \\
\text { recognition task (RT) }\end{array}$ & $\begin{array}{l}\text { hearing } \\
\text { CODAs }\end{array}$ & $\begin{array}{l}\mathrm{L} 2 \rightarrow \mathrm{L} 1 ; \\
\text { perception }\end{array}$ & $\begin{array}{l}\text { sign iconicity effects } \\
\text { cross-lang interaction }\end{array}$ \\
\hline $\begin{array}{l}\text { Bishop \& Hicks } \\
(2005)\end{array}$ & $\begin{array}{l}\text { analysis of written data } \\
\text { (emails to a private } \\
\text { CODA listserv) }\end{array}$ & $\begin{array}{l}\text { hearing } \\
\text { CODAs } \\
\text { (ASL-Eng.) }\end{array}$ & $\begin{array}{l}\text { Engl. } \\
\text { production }\end{array}$ & $\begin{array}{l}\text { written English provides } \\
\text { ASL influence }\end{array}$ \\
\hline $\begin{array}{l}\text { Casey \& Emmorey } \\
(2008)\end{array}$ & $\begin{array}{l}\text { production of co- } \\
\text { speech gestures and } \\
\text { signs during Engl. } \\
\text { story-telling }\end{array}$ & $\begin{array}{l}\text { hearing } \\
\text { CODAs } \\
\text { (ASL-Eng.) }\end{array}$ & $\begin{array}{l}\text { Engl. } \\
\text { production }\end{array}$ & $\begin{array}{l}\text { bimodality influenced the } \\
\text { iconicity, perspective, } \\
\text { and handshape of co- } \\
\text { speech gestures. }\end{array}$ \\
\hline $\begin{array}{l}\text { Emmorey et. al } \\
(2008)\end{array}$ & $\begin{array}{l}\text { production of co- } \\
\text { speech gestures and } \\
\text { code-blends during } \\
\text { Engl. story-telling and } \\
\text { conversation. }\end{array}$ & $\begin{array}{l}\text { hearing } \\
\text { CODAs } \\
\text { (ASL-Eng.) }\end{array}$ & $\begin{array}{l}\text { Engl. } \\
\text { production }\end{array}$ & $\begin{array}{l}\text { produce more code- } \\
\text { blends, when addressee } \\
\text { is also bilingual }\end{array}$ \\
\hline $\begin{array}{l}\text { Pyers \& Emmorey } \\
(2008)\end{array}$ & $\begin{array}{l}\text { Engl. conversation } \\
\text { about hypothetical } \\
\text { situations. }\end{array}$ & $\begin{array}{l}\text { hearing } \\
\text { CODAs } \\
\text { (ASL-Eng.) }\end{array}$ & $\begin{array}{l}\text { Engl. } \\
\text { production }\end{array}$ & $\begin{array}{l}\text { bilinguals produced more } \\
\text { ASL-appropriate facial } \\
\text { expressions and aligned } \\
\text { them to English clauses }\end{array}$ \\
\hline $\begin{array}{l}\text { Morford et al. } \\
(2011)\end{array}$ & $\begin{array}{l}\text { Engl. semantic decision } \\
\text { task (RT); phonological } \\
\text { relatedness in ASL } \\
\text { translation }\end{array}$ & \begin{tabular}{|l} 
deaf \\
bilinguals \\
(ASL-Eng.)
\end{tabular} & $\begin{array}{l}\mathrm{L} 2 \rightarrow \mathrm{L} 1 ; \\
\text { perception }\end{array}$ & $\begin{array}{l}\text { reaction time to +se/+ph } \\
\text { words was faster than to } \\
+ \text { se/-ph; rejection of -se/ } \\
+ \text { ph was slower than to - } \\
\text { se/-ph }\end{array}$ \\
\hline $\begin{array}{l}\text { Ormel et al. } \\
(2012)\end{array}$ & $\begin{array}{l}\text { word-pic-verification } \\
\text { task (RT) }\end{array}$ & \begin{tabular}{|l} 
deaf bilingual \\
children \\
(NGT-Dutch) \\
\end{tabular} & $\begin{array}{l}\mathrm{L} 2 \rightarrow \mathrm{L} 1 ; \\
\text { perception }\end{array}$ & $\begin{array}{l}\text { inhibition effect for phon. } \\
\text { related pairs }\end{array}$ \\
\hline $\begin{array}{l}\text { Shook \& Marian } \\
(2012)\end{array}$ & $\begin{array}{l}\text { eyetracking, visual- } \\
\text { world paradigm }\end{array}$ & \begin{tabular}{|l} 
hearing \\
bilinguals \\
(ASL-Eng.) \\
\end{tabular} & $\begin{array}{l}\mathrm{L} 2 \rightarrow \mathrm{L} 1(?) \\
\text { perception }\end{array}$ & $\begin{array}{l}\text { competitors with phon. } \\
\text { relation in ASL were } \\
\text { looked at longer }\end{array}$ \\
\hline $\begin{array}{l}\text { Kubus et al. } \\
(2014)\end{array}$ & $\begin{array}{l}\text { Germ. semantic } \\
\text { decision task (RT); } \\
\text { phonological } \\
\text { relatedness in DGS } \\
\text { translation }\end{array}$ & $\begin{array}{l}\text { deaf } \\
\text { bilinguals } \\
\text { (DGS-Germ.) }\end{array}$ & $\begin{array}{l}\mathrm{L} 2 \rightarrow \mathrm{L} 1 ; \\
\text { perception }\end{array}$ & $\begin{array}{l}\text { rejection of -se/+ph was } \\
\text { slower than to -se/-ph }\end{array}$ \\
\hline
\end{tabular}


While these studies either found an interaction between sign and spoken language rather by coincidence (Treiman \& Hirsh-Pasek, 1983; Hanson \& Feldman, 1989; Grote \& Linz, 2003), or mainly focused on the influence of sign language knowledge during spoken language production (Bishop \& Hicks, 2005; Casey \& Emmorey, 2008; Emmorey et al., 2008; Pyers \& Emmorey, 2008), only few investigated underlying cross-modal co-activation during L2 processing (Morford et al., 2011; Ormel et al., 2012; Shook \& Marian, 2012; Kubus et al., 2014). No study up to date investigated potential co-activation of second language word representations during native sign language processing.

\subsubsection{The present study}

Since deaf bimodal bilinguals concurrently navigate within a spoken language world, both of their languages - the sign language used with partners, family, and friends and the spoken language used with work colleagues and in public life - are constantly high activated. Switching between both language modalities, i.e. communicating in a sign language on the one hand and lip reading, oral articulating and writing on the other hand, is omnipresent. Thus, the question is, does the processing of L1 signs also co-activate covert orthographic and/or phonologic representations of L2 words? Although congenitally deaf people ${ }^{10}$ have no auditory representation of spoken words, we nevertheless assume that deaf people have a "phonological" or rather sublexical representation of spoken words. That is a collective knowledge on how the spoken word is articulated (articulatory information), how it is written (orthographic information) and how the pronunciation of the word by others looks like (visual information). Therefore, in the design of our study, we did

10 Congenitally deaf children with deaf parents learn a sign language as their native language by their parents and a spoken language as second language via visual mouth representations of spoken words and orthographic representations of written words. Nonetheless, the majority of Deaf people (i.e. 90\%) either grew up with hearing parents and/or was born hearing and deafened due to an early hearing loss. Their phase of language acquisition (sign language and spoken language) is rather diverse. 
not separate between orthographic and phonological representations of spoken words.

A second aim of our study was to test cross-modal language co-activation during whole sentence processing, instead of during single word processing. For spoken language processing, Van Petten (1995) reviews several ERP studies that provide compelling evidence for differences in sentences processing versus isolated word processing. For example, the processing of low frequent words compared to the processing of high frequent words in an isolated presentation will lead to larger N400s (cf. Rugg, 1990; Van Petten \& Kutas, 1990). That same frequency effect is suppressed when high and low frequent words are presented in meaningful sentence endings (cf. Van Petten, 1995: p. 519). Also, Bornkessel-Schlesewsky et al. (n.d.) point out that ERP pattern of natural reading compared to ERP pattern of reading single words in rapid-serial-visual-presentation (RSVP) can be qualitatively different. This is justified by the absence of parafovial preview during RSVP reading. During natural reading, participants have the ability to grasp, for example, frequency information of the upcoming word(s) parafovially (cf. Kretzschmar, 2010, Kretzschmar et al., 2009). This leads to very early frequency effects in the ERP pattern, which can be suppressed when parafovial preview is not available. Similarly, for processing the visual signal of sign language sentences, signers not only use the input information of lexical signs (as defined from sign onset to sign offset), but they also use input information of the transition phases prior to lexical signs (see Chapter 1 and Hosemann et al.; 2013). Transition phases provide sub-lexical information, like, e.g., a movement trajectory of the hand(s) towards a target location in signing space, that are used for balancing top-down expectations with buttom-up input. Thus, in order to investigate cross-modal co-activation in natural sign language comprehension, in this study, we examine prime-target pairs presented in full sentences instead of as isolated signs. We asked, whether signers co-activate orthographic/ phonological representations of German words (L2) during the processing of sentences in their native sign language DGS (L1). 
In the present study, we followed two main objectives: First, to investigate phonological priming in sign language within whole sentence processing; and second, to examine cross-modal cross-language activation of L2 orthographic/phonological representations during L1 sign language processing. To test our research questions, we presented deaf native signers videos of semantically and grammatically acceptable sentences in German Sign Language (DGS) while we recorded their Electroencephalogram (EEG). Within the sentences two signs functioned as prime and target. In the within language priming condition, prime and target signs phonologically overlapped in three out of the four phonological parameters. Thus, they differed in either handshape, movement, location or orientation of the sign (for example, the signs STORE and ANIMAL differ only in their movement, see Figure 2.1).
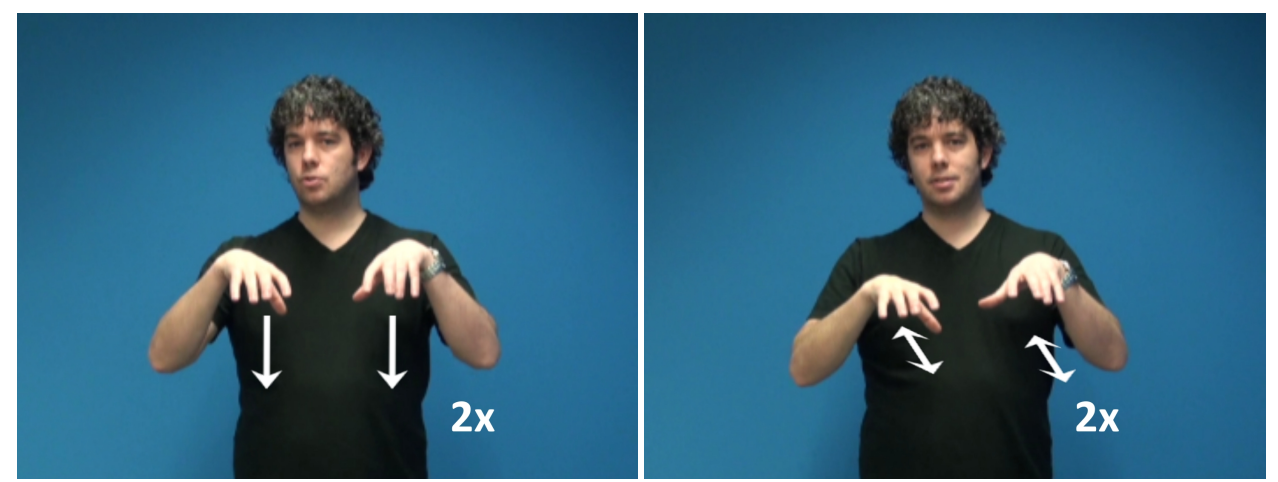

Figure 2.1 Video stills of the signs STORE (left) and ANIMAL (right). The distinctive parameter is the movement: STORE has a reduplicated up-and-down movement, while ANIMAL has an alternating back-and-forth movement.

In the cross-modal cross-language priming condition, prime and target were phonologically unrelated signs (no phonological parameter overlapped). However, their German translations were phonological and orthographic minimal pairs. For example, the signs MOTHER and BUTTER have no phonological overlap in DGS, but the German translations differ only in the onset grapheme and phoneme: 'Mutter' - 'Butter'). Note that all items in this condition form minimal pairs with respect to German orthography and 
phonology; thus, in our stimulus set, we did not differentiate between orthographic and phonological overlap. In the control conditions, sentences were similar to their within language and cross-language counterparts, except for the prime that was now phonologically and orthographically unrelated to the target. Furthermore, in all conditions, prime and target were semantically unrelated.

Using an auditory phonological priming task with spoken languages, Praamstra et al. (1994) showed that ERPs to targets in the phonologically related condition (where prime and target overlapped phonologically) were less negative (across the critical N400 window) compared to targets in the phonologically unrelated condition. They explain this finding by suggesting that parts of the target word were already pre-activated by the prime word. We expected similar results for the within language (DGS) related primetarget signs. Since prime and target were minimal pairs and differed only in one phonological parameter, the other phonological parameters should be already pre-activated by the prime sign. For example, as can be seen in Figure 2.1, STORE and ANIMAL are both two handed signs with a "bent-5" handshape realized in neutral signing space. They only differ in their movement path: while STORE has a reduplicated up-and-down movement, ANIMAL has an alternating back-and-forth movement. Hence, phonological features like handshape, orientation and location of the target ANIMAL are previously realized by the prime STORE. This is expected to result in a processing advantage of the related target and, thus, in a lower amplitude of the N400 component timelocked to the target.

Our expectations regarding the cross-language priming condition were similar. If signers co-activate orthographic/phonological representations of corresponding German words during sign processing, we would also expect to find a reduced $\mathrm{N} 400$ for target signs that overlap with the prime in their German translation. This expectation is based on the premise that, were participants to co-activate cross-language representations of sign language 
stimuli, then parts of the orthographic/phonologic German representation of the target have been previously activated during processing of the prime.

\subsection{Materials and methods}

\subsubsection{Participants}

A total of 15 congenitally deaf native signers of DGS participated in the experiment as paid volunteers after giving written informed consent ( 9 male, 6 female). All participants were right-handed, had normal or corrected-tonormal vision and reported no history of neurological disorders. Their ages ranged from 24 to 48 years (mean 31.8; sd 7.41) and they came from different parts of Germany. All signers had deaf parents or DGS input before the age of three (AoA L1: 0-3; mean 0.83; sd 1.27). A questionnaire on individual metadata that was administered after the experiment stated that participants learned written German at an average age of 41/2 years (AoA L2: 2-7; mean 4.53; sd 1.3). On a 1-10 scale, participants rated their proficiency in written German on average 6.73 (range 4-10; sd 1.28). Most of them regularly write German during work contexts, in emails and via chat. Tabel 2.2 gives an overview of participant's metadata.

For the hearing control group, 14 monolingual native German speakers were recruited around the university campus ( $3 \mathrm{~m}, 11 \mathrm{f}$; age 19-32; mean 22.5; sd 3.82). They reported full hearing and had normal or corrected-to-normal vision. None of them had any previous contact to German Sign Language.

Table 2.2 Overview of participants' metadata. Proficiency was self-evaluated on a 1-10 scale; "m" = means; standard deviations are shown in parentheses.

\begin{tabular}{|l|l|l|l|l|l|}
\hline & \multicolumn{1}{|c|}{ age } & \multicolumn{1}{|c|}{ AoA DGS } & \multicolumn{1}{|c|}{ AoA German } & $\begin{array}{l}\text { proficiency } \\
\text { written Ger. }\end{array}$ & \multicolumn{1}{c|}{$\begin{array}{c}\text { usage } \\
\text { written Ger. }\end{array}$} \\
\hline $\begin{array}{l}\text { deaf } \\
\text { signers } \\
(\mathrm{N}=15)\end{array}$ & $\begin{array}{l}24-48 \text { years; } \\
\text { m: } 31.8(7.41)\end{array}$ & $\begin{array}{l}0-3 \text { years; } \\
\text { m: 0.83 (1.27) }\end{array}$ & $\begin{array}{l}2-7 \text { years; } \\
\text { m: } 4.53(1.3)\end{array}$ & $\begin{array}{l}4-10 ; \\
\text { m: 6.73 (1.28) }\end{array}$ & $\begin{array}{l}\text { work, mail, chat, } \\
\text { SMS, communi- } \\
\text { cation with hearing, }\end{array}$ \\
\hline $\begin{array}{l}\text { hearing } \\
\text { controls } \\
(\mathrm{N}=14)\end{array}$ & $\begin{array}{l}19-32 \text { years; } \\
\text { m: } 22.5(3.82)\end{array}$ & no DGS & 0 years & n/a & n/a \\
\hline
\end{tabular}




\subsubsection{Materials}

The materials were discussed, developed and video recorded in collaboration with two deaf DGS informants (one female, one male). The stimulus material consisted of a total of 160 sentences, 40 sentences per condition (see Appendix A for a complete stimulus list). Sentences began with DGS typical sentence beginnings: either a topic construction (TOPIC SOCCER ...), a temporal construction (THIS YEAR ...), or a location (SUPERMARKET INDEX ...). Sentence beginnings were subsequently followed by the prime, an intermediate index sign, and the target. They completed with a sentence end.

In the DGS priming condition (i.e. within language priming), prime and target signs were minimal pairs in that they overlapped in three phonological parameters. Thus, they differed either in handshape, movement, location or orientation of the sign (cf. the example of STORE - ANIMAL in Figure 2.1). Sentences in the DGS control condition were identical to their DGS priming counterparts, except for the prime, which was phonologically unrelated to the target in DGS as well as in the German translation (CHURCH - ANIMAL). In the German priming condition (i.e. cross-modal cross-language priming), prime and target had no phonological overlap as signs, but their German translation equivalents were orthographic and phonologic minimal pairs in that they only differed in the first grapheme/phoneme (MOTHER - BUTTER, 'Mutter' 'Butter'). Again, sentences in the German control condition were identical to their counterparts, except for the prime, which was phonologically unrelated to the target in DGS as well as in the German translation (FATHER - BUTTER, 'Vater' - 'Butter'). Prime and target were semantically unrelated across all conditions. Note that by changing prime-signs between conditions we could keep target signs identical across the related and the control conditions. Any differences in target processing across primed and unrelated conditions can thus be attributed to the different relation between primes and targets. In (3) we present example sentences for each condition. 
(3) a. DGS priming condition:

DGS: USUALLY STORE IX ANIMAL ALLOWED-NEG VISIT

German: 'Normalerweise sind in Geschäften keine Tiere erlaubt.'

English: 'Usually, animals are not allowed to enter stores.'

b. DGS control condition:

DGS: $\quad$ USUALLY CHURCH IX ANIMAL ALLOWED-NEG VISIT

German: 'Normalerweise sind in Kirchen keine Tiere erlaubt.'

English: 'Usually, animals are not allowed to enter churches.'

c. German priming condition:

DGS: REFRIGERATOR POSS ${ }_{1}$ MOTHER IX BUTTER TAKE-OUT

German: 'Meine Mutter holt Butter aus dem Kühlschrank.'

English: 'My mother takes out the butter from the refrigerator.'

\section{d. German control condition:}

DGS: REFRIGERATOR POSS ${ }_{1}$ FATHER IX BUTTER TAKE-OUT

German: 'Mein Vater holt Butter aus dem Kühlschrank.'

English: 'My father takes out the butter from the refrigerator.'

Note that IX is a so-called index or pointing sign, which is used for referential anchoring of locations within signing space.

Stimulus sentences were signed by a male deaf informant and recorded with a HDR-XR 550E full-HD camera (25 frames / second). For cutting and editing the material, we used the video editing software application Adobe Premiere Pro, so that each stimulus sentence consisted of an un-manipulated video (i.e. the stimulus material was not spliced, lengthened or shortened). Videos had a width of 720 pixels and a height of 576 pixels (corresponding to a size of approximately 25 by $20 \mathrm{~cm}$ on screen). The DGS sentence was preceded by 2 seconds in which the signer remained still before starting to sign. After the end of the sentence, the signer again remained motionless for 1 second. On 
average the stimulus videos had a length of 9.34 seconds ( $s d 1.04)$. The prime signs started on average 4.404 seconds into the video (sd 0.83) and had an average length of 0.532 seconds (sd 0.13 ). The target signs started on average 6.057 seconds into the video ( $\mathrm{sd} 0.86$ ) and had an average length of 0.505 seconds ( $\mathrm{sd}$ 0.13). The intermediate times between prime offsets and target onsets added up to an average length of 1.122 seconds ( $\mathrm{sd} 0.19$ ). This results from the length of the intermediate INDEX sign and the preceding and subsequent transition phases between signs. Transition phases can be comparatively long and last up to $200 \mathrm{~ms}$ (cf. Hosemann et al., 2013; Jantunen, 2013). Investigating these intervals between primes and targets across conditions showed no significant differences in length: average length of intervals in DGS priming: 1.149 [0.22], average length of intervals in DGS control: 1.074 [0.21], average length of intervals in German priming: 1.137 [0.18], average length of intervals in German control: 1.128 [0.15]; $\mathrm{p}=0.28$ ). Also, none of the other average time measures differed between related and control conditions ( $p s>0.16$ ). In addition to the stimulus sentences, we recorded 8 practice sentences that were similar in structure to the stimulus sentences.

We instructed our informant to sign as natural as possible. For clarity, signing was slightly slower compared to natural conversation speed, but included non-manual action when appropriate, except for mouthing. Nonmanual components (like movements of the head and upper body, facial expressions, eye movement, and mouth actions) constitute an essential grammatical part of sign languages. The term "mouthing" refers to the silent pronunciation of the corresponding spoken word simultaneous to producing a sign. We wanted to keep the processing of a sign merely to the visual image of the manual sign as input. Thereby, we wanted to exclude a co-activation of the German translation equivalent via the processing of the overt visual image of the mouthing. Thus, we instructed our informant to use no mouthing on prime and target signs. 
The missing mouthing on prime and target signs increased the potential ambiguity of the signs. In order to test whether participants activated the exact German minimal pairs we intended them to activate, we conducted a post-experimental translation task. For this, participants saw the 40 videos of the German related condition and had to translate the content of each video into written German (see Section 2.2.5 for a more detailed explanation).

\subsubsection{Procedure}

Participants were seated in a dimly lit experimental room in front of a $92 \times 50 \mathrm{~cm}$ TV screen with a distance of approximately one meter from the screen. In order to exclude any spoken German influence during the procedure of the experiment, all conversation (before, during, and after the experiment) was held by a deaf research assistant in DGS. After giving written consent to the experiment, participants saw an introduction video explaining the assignment of the experiment in DGS. We asked participants to simply watch the following videos and to answer the interspersed yes/no-questions regarding the content of the previous videos. To answer the question, participants had to press a corresponding button on an X-Box controller.

Each participant was first presented with two practice blocks. Each practice block included 8 trials presenting a sentence video, and one trial presenting a yes/no-question. Trials were separated by $1000 \mathrm{~ms}$ during which a blank screen was shown. Sentences and questions used in the practice blocks were not part of the critical stimulus material. After completion of the practice blocks and clarification of any questions, the experiment started. The experimental session was split into 4 blocks, each containing 40 critical sentence trials interspersed with five trials where participants were asked a question related to the content of the previous sentences. Thus, each block contained 10 sentences of each condition (DGS priming, DGS control, German priming, German control). Sentences were assigned to blocks in a pseudorandomized fashion so that target signs were not repeated within blocks. 
Order of blocks was counterbalanced across participants, while presentation of sentences within blocks was randomized. Question trials - where participants were asked a question about the content of a previous sentence were inserted after every 8th test trial. Sentence trials were presented automatically and separated by $1000 \mathrm{~ms}$ during which a blank screen was shown. Sentence trials after question trials would start $1000 \mathrm{~ms}$ after a response button was pressed. No feedback on the accuracy of the response was given. After every block, participants could take a break and continue the experiment by pressing a button on the response box.

After finishing the experimental session, participants had time to wash their hair before filling out the post-experimental translation task. At the end, participants provided their personal metadata about their age of acquiring DGS and German, and their (written) German proficiency and usage.

The hearing control group participated in an identical experimental procedure. Practice video trials as well as critical video trials were the same as for deaf participants. The only difference between hearing and deaf experimental setups was that comprehension questions for hearing participants were translated into written German. This was done to provide both groups with the same assignment, although we did not expect hearing participants to understand the DGS videos. Also, hearing participants did not have to fill-out the translation task.

\subsubsection{EEG recording}

EEG data were recorded using the Biosemi Active Two Amplifier system. We placed $32 \mathrm{Ag} / \mathrm{AgCl}$ electrodes according to the international 10-20 system, and recorded at a sampling rate of $2048 \mathrm{~Hz}$. Electrode offsets were kept $<20 \mu \mathrm{V}$. EEG recordings were referenced offline to the average left and right mastoid reference. The electrooculogram (EOG) was recorded for each participant from three electrodes, one at the outer canthi of each eye (horizontal EOG), and one below the left eye (vertical EOG). 


\subsubsection{EEG data preprocessing and statistical analysis}

The raw EEG data was filtered offline with a $0.01 \mathrm{~Hz}$ high-pass and a $30 \mathrm{~Hz}$ low-pass filter. Single subject averages were calculated per condition and electrode between -200 and $1000 \mathrm{~ms}$ relative to the trigger sign onset (see below for an explanation of trigger placing). Trials that contained eye blinks and other artifacts were rejected using a $120 \mathrm{~Hz}$ amplitude cut-off threshold. Note that three out of the 15 deaf participants had to be excluded from further analysis, due to excessive eye movement artifacts and/or major EEG drifts. No hearing participant from the control group had to be excluded. Thus, the final grand averages were computed over 12 deaf participants and for the control group over 14 hearing participants.

For each deaf participant trials were further excluded based on the translations given in the translation task. In the post-experimental translation task, participants saw all 40 videos of the condition German related (prime and target are phonologically unrelated as signs, but minimal pairs in their German translation; i.e. Mutter - Butter), and had to write down the content of each video. In this experiment, we focused mainly on the cross-language activation from DGS to written German. The translation task was meant to ensure that participants actually activated the German minimal pairs we intended them to activate. Signs in DGS do not necessarily have a one-to-one translation in German, but can rather be ambiguous. For example, the DGS sign CUP can also mean 'drink coffee out of a cup' or even only 'coffee'. With the sentence "KITCHEN STORE WOMAN CHECKOUT IX CUP PAY" ('In a kitchenstore, a women pays for her cup at the checkout'), we intended the activation of the German minimal pair Kasse (checkout) and Tasse (cup). Nevertheless, most participants translated the sentence as "In a kitchen-store, a women pays for her coffee (Kaffee) at the checkout." Thus, we decided to exclude sentences with these unintended translations of prime or target from the analysis. On average, deaf participants translated prime and target as intended in $51,67 \%$ of the cases (mean of "correct" translated sentences: 20.67; range 13-25; sd 3.47). The comparatively high number of "false" 
translated videos can be explained by two factors: First, we were relatively strict and only accepted those translations as correct that we intended when creating the stimuli. Thus, we excluded semantically related words, like e.g. hypernyms as "flower" instead of the expected "rose". Second, signs have a higher contextual ambiguity compared to German words (see the previously mentioned CUP versus COFFEE example). Those "false" translations had to be excluded as well. At the end, a total of 248 out of 480 items for the German priming and their German control counterparts entered the analysis.

Thus, single subject averages were calculated including all artifact-free trials for the hearing participants; for deaf participants, we calculated all artifactfree trials of conditions DGS priming and DGS control, and all artifact-free and "correctly" translated trials of conditions German priming and German control.

Deriving event-related potentials from an ongoing natural signing stream leads to the challenge of identifying the correlating trigger point. Previously, Hosemann et al. (2013) tested in an ERP study three different trigger positions within the signing stream to identify the trigger that was related to the ERP effect (compare Chapter 1). One trigger was placed at the sign onset (i.e. all phonological parameters are clearly identifiable); two triggers were time-locked to information sources in the transition phase preceding the critical sign. The results showed that the processing of the sign is already ongoing within the transition phase prior to the sign onset. Thus, in terms of analyzing ERPs in ongoing signing streams, the preceding transition phase of a target sign has to be considered as being part of the sign. Therefore, we apply no baseline corrections relative to the trigger time-locked to the sign onset. This baseline phase includes the preceding transition phase, in which the processing of the sign is already ongoing. ${ }^{11}$ Trigger "sign onset" is defined as the first frame of the target sign in which the target handshape reaches its target location, right before the sign moves to its final position (i.e. the three

11 For those interested in an ERP analysis with a baseline correction prior to the trigger position, we also provide baseline corrected results in Footnote 13. 
phonological parameters handshape, orientation and location are fully formed). In sign language linguistics this moment is often classified as the beginning of a sign (e.g., Gutiérrez et al. 2012a). A trigger within the transition phase previous to the sign onset would be too early for a full identification of all phonological parameters. Thus, epochs were defined as starting $200 \mathrm{~ms}$ prior and ending $1000 \mathrm{~ms}$ after the trigger sign onset. Figure 2.2 illustrates trigger positioning within an ongoing signing stream according to the moment of sign onset.

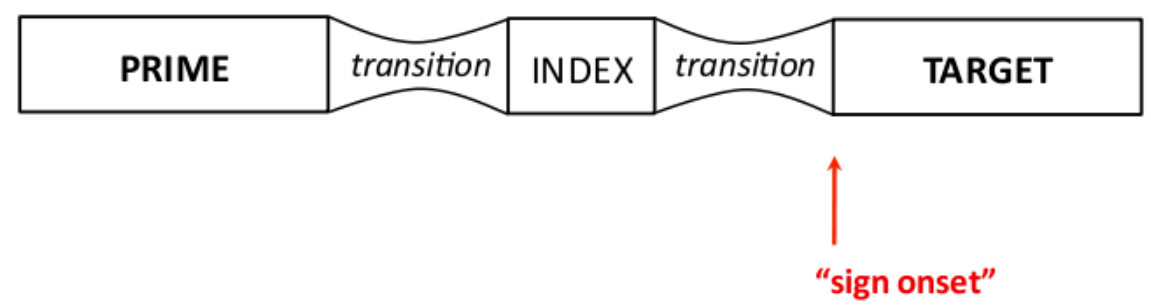

Figure 2.2 Schematic illustration of a signing stream, including transition phases and the time point of trigger "sign onset".

For the statistical analysis, repeated analyses of variance (ANOVAs) were calculated with the statistical program SPSS, for the factors GROUP (deaf vs. hearing); CONDITION (DGS vs. German); PRIMING (priming vs. control); LATERALITY (left hemisphere vs. right hemisphere); and REGION. Lateral regions of interests were separated into three regions, i.e., fronto-central (F3, F4, FC1, FC2), centro-parietal (C3, C4, CP1, CP2), and parietal-occipital (P3, P4, P03, P04). A separate midline analysis examined brain potentials across midline electrodes $\mathrm{Fz}, \mathrm{Cz}$, and Pz. Following visual inspection of the grand average, we analyzed a 450-650 ms time window past sign onset. The statistical analysis was carried out in a hierarchical manner. Thus, only significant interactions $(\mathrm{p}<0.05)$ were further analyzed. Where appropriate, a Greenhouse-Geisser correction was applied (Greenhouse \& Geisser, 1959). 


\subsection{Results}

\subsubsection{Behavioral data}

For general attention control, participants had to answer a yes/no-question regarding the content of one of the previously seen videos. The total of 160 critical videos was divided in 20 blocks of eight videos, each block finishing with a question video. Thus, 20 questions had to be answered, thereof 10 "yes"-questions and 10 "no"-questions. In 72,5\% (174/240) participants responded with the correct answer; and in 27,5\% (66/240) the answers were not as intended. In fact, one participant missed all 20 questions, maybe because of confusion of response buttons. Excluding this participant from the behavioral data analysis, the percentage distribution of responses is $79.9 \%$ hits to $20.91 \%$ misses (i.e. 174 intended answers compared to 46 erroneous answers, out of 220 total).

\subsubsection{ERP data}

In Figure 2.3 we present grand averages of ERPs time-locked to the sign onset of the target sign for within language priming conditions (DGS priming) as well as for cross-language priming conditions (German priming). As can be seen from the graphs for DGS and German priming conditions, target signs in control conditions (marked in dark and bright blue) engendered a more negative ERP waveform compared to target signs in priming conditions (marked in dark and bright red). Following visual inspection, this effect persists approximately between 400-650 ms for the DGS priming condition and between 250-700 ms for the German priming condition. However, a statistical analysis in successive $50 \mathrm{~ms}$ time windows revealed the effect to be significant between 450-650 ms for both priming conditions (shaded in grey). 


\section{(A) DGS priming condition}

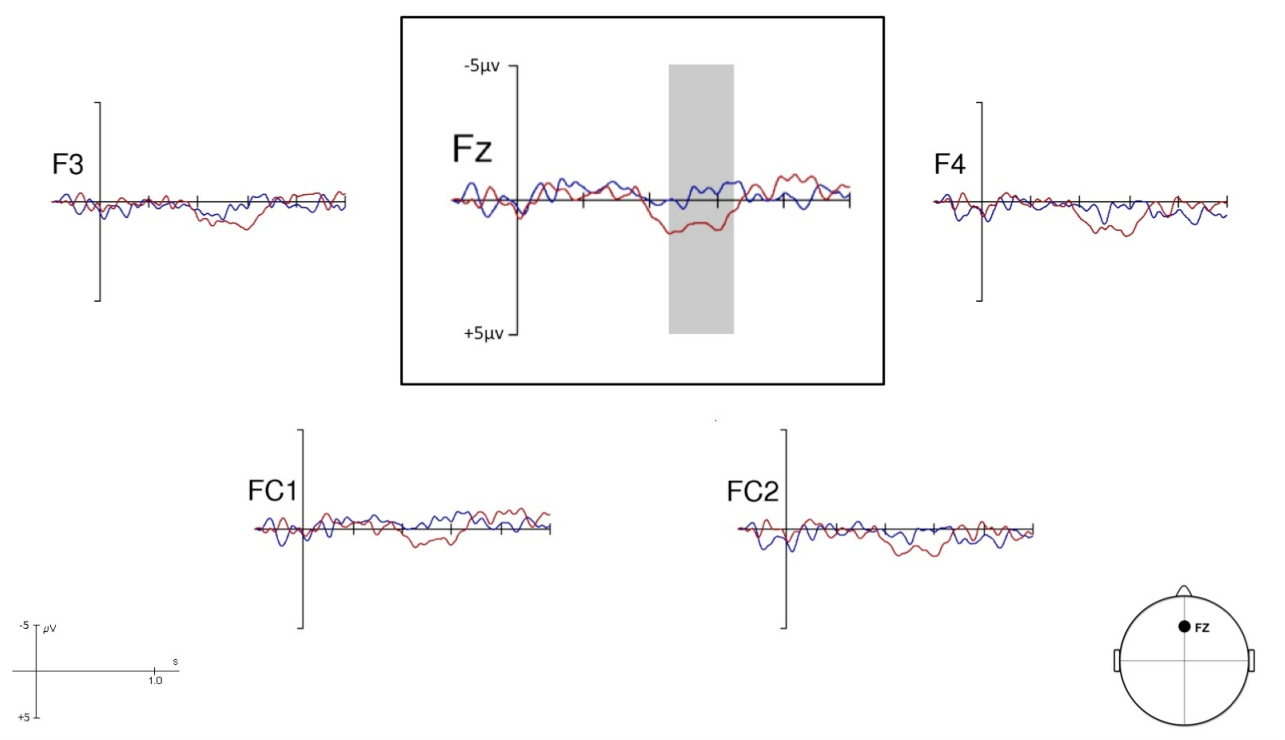

(B) German priming condition

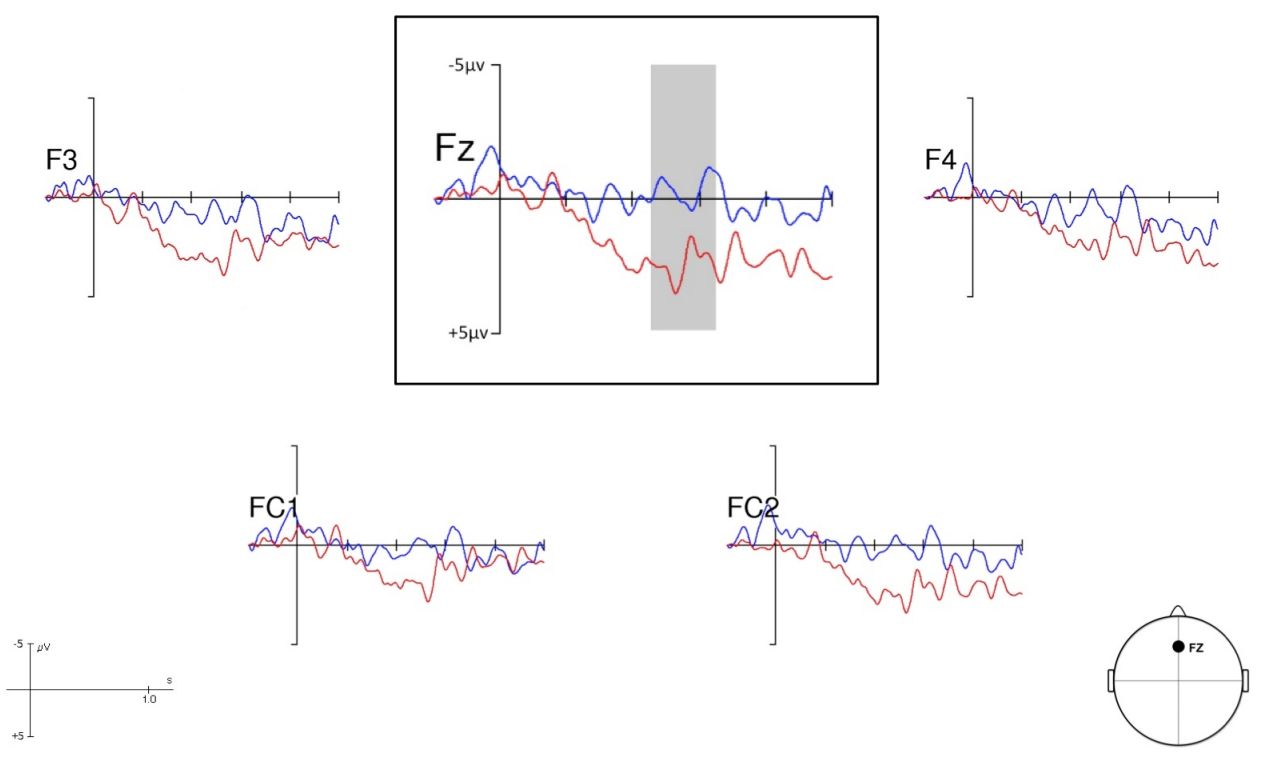

Figure 2.3 Grand average ERPs for (A) target signs in DGS priming condition (primed targets $=$ dark red, unprimed control targets $=$ dark blue) and for (B) target signs in German priming condition (primed targets = bright red, unprimed targets = bright blue). Negativity is plotted upwards. The related trigger position is the sign onset. For visual presentation of the plots we used a 0.1-20 Hz display filter. 
The results indicate a reduced negative polarity for primed target signs compared to overall unprimed target signs. ${ }^{12}$ For the DGS priming condition, the effect was predominantly spread over frontal electrodes, while the German priming condition engendered a more broadly distributed effect. Note that grand averages of the German priming condition, which appear somehow noisy, include less critical items than grand averages of the DGS priming condition, due to the exclusion of "falsely" translated prime-target pairs from the post-experimental translation task. Further ERP figures with a more extensive selection of electrodes for both priming conditions are presented in Appendix B.

An omnibus ANOVA of the deaf group and the hearing control group with the factors GROUP (deaf vs. hearing), CONDITION (DGS vs. German), PRIMING (priming vs. control), LATERALITY (left hemisphere vs. right hemisphere), and REGION revealed a main effect of priming $(F(1,25)=9.60, p=0.005)$, and an interaction of priming and group $(F(1,25)=7.38, p=0.01)$ for the lateral electrodes, as well as for the midline electrodes (main effect of priming: $F(1,25)=10.04, \mathrm{p}<0.005$; interaction of priming and group: $F(1,25)=8.61$, $p=0.007)$. Thus, we separately analyzed the data of the deaf and the hearing group. The deaf group showed in an overall analysis a main effect of priming (lateral: $F(1,11)=12.99, p=0.004$; midline: $F(1,11)=13.97, \mathrm{p}=0.003$ ). No other main effects or interactions reached significance ( $p s>0.1$ ). The absence of an interaction between priming and condition suggests that both the DGS and German related conditions elicited a priming effect in our deaf participants. Post-hoc comparisons confirmed that the mean amplitudes of the EEG signal in the time window 450 to $650 \mathrm{~ms}$ of the priming conditions are significantly reduced compared to the mean amplitudes of the control conditions. Thus, mean ERP amplitudes of targets that were preceded by a prime that either shared phonological parameters with the target in DGS or

${ }^{12}$ An investigation of the raw EEG signal filtered offline with a $0.3 \mathrm{~Hz}$ high-pass and a 20 $\mathrm{Hz}$ low-pass filter (at a $50 \mathrm{~Hz}$ amplitude cut-off) revealed the same negativity effect for unprimed control target signs compared to primed target signs (in both DGS and German priming condition). 
that was orthographically and phonologically related in its German translation equivalent were significantly less negative than their unrelated controls. These results suggest that deaf native signers co-activated not only phonologically related signs in their native sign language DGS during processing of signs embedded in sentences, but that they also concurrently activated translations of the signs in their second language German. ${ }^{13}$

The corresponding analysis of the data of the hearing control group showed no significant main effects or interactions. This suggests that hearing participants without knowledge of DGS do not show any differences between conditions, i.e. no priming effects. This excludes low-level visual similarities as a basis for the priming effects observed in the deaf participants.

\subsection{Discussion}

The main objectives of the current study were, first, to test sign priming effects within natural sign language sentence processing (instead of single sign processing) with an online method; and second, to test cross-modal cross-language activation of L2 orthographic/phonological representations during L1 native sign language processing (instead of L1 activation during L2 processing). We investigated ERP responses to the processing of a target sign following either a phonologically related prime sign in DGS (within language priming), or following a prime sign that was phonologically unrelated in DGS, but had an orthographic/phonological overlap with the target in the German translation (cross-language priming). Prime and target, presented in DGS sentences, were semantically unrelated. For both conditions (each contrasted with a corresponding control condition that included an overall unrelated prime), we found a priming effect. The electrophysiological signal was

${ }^{13} \mathrm{An}$ analysis including a baseline correction -200 to $-100 \mathrm{~ms}$ prior to the trigger position revealed no difference in the overall effects. The deaf group showed an overall main effect of condition (DGS vs. German) and of priming (priming vs. control), but no interaction: main effect CONDITION: lateral electrodes $F(1,11)=7.67, p=0.018$, midline electrodes $F(1,11)=5.43, p=0.04$; main effect of PRIMING: lateral electrodes $F(1,11)=15.28, p=0.002$, midline electrodes $F(1,11)=14.57, p=0.003$. 
significantly less negative in the DGS and in the German priming condition compared to control conditions, respectively. This suggests that features of the target sign were pre-activated by the prime sign resulting in lower processing costs for related compared to unrelated target signs. Interestingly, this holds not only for target signs that are preceded by a sign-phonologically related prime (like STORE and ANIMAL which solely differ in the parameter movement), but also for prime-target pairs that have in fact no overlap in sign phonology but that overlap in the orthography/phonology of their German translations (like MOTHER and BUTTER, 'Mutter - Butter'). Thus, we argue on the one hand that overt phonological priming effects can be observed in natural sign language sentence processing; and on the other hand, that deaf native signers co-activate their second language orthographic/phonological representations during native sign language processing.

An underlying theoretical question behind studies on bimodal cross-language co-activation asks how this co-activation between elements of two languages of different modalities can be mediated, especially with respect to a missing overt phonological similarity between sign and word. In other words, how are the representations of signs and words linked? An appealing and often suggested explanation is the mediation via an independent but shared semantic representational node. Another explanation assumes a direct, asemantic associative link between sign and word representation (both discussed, e.g., by Morford et al., 2011, Ormel et al., 2012, and Shook \& Marian, 2012). In the following, we will review both approaches before we come to a third possible explanation, assuming an asemantic link between sign and word representation via a shared representation of the corresponding mouthing.

Previous studies on cross-modal cross-language activation with deaf bimodal bilinguals found that sign-phonological representations of L1 were coactivated during the processing of single written words in L2 (Morford et al., 2011; Ormel et al., 2012; Kubus et al., 2014). For instance, Morford et al. (2011) found significantly faster response times to semantically related 
prime-target word pairs that were also phonologically related as sign translations. In contrast, Ormel et al. (2012) report a significant delay in response times for those word-picture pairs that had phonologically overlapping sign translations. They explain this inhibition effect by competition processes between phonological neighbor signs during lexical access. Reading the Dutch word (e.g. "hond", dog) co-activates its sign translation (DOG) and by that also the signs phonological neighbors. This includes the phonologically related sign translation of the target picture (CHAIR). These competing items lead to an inhibition of the lexical decision.

Irrespective of finding facilitation or inhibition effects, an often mentioned and discussed explanation for cross-modal co-activation is one via an independent semantic representation that is connected with the lexical representation of the sign as well as with the lexical representation of the (written) word. According to this "semantic connection explanation", the visual impression of a letter string (or specific sound waves) activates via the sub-lexical level the lexical level of the word representation. This then activates the corresponding semantic/conceptual representation that is shared by both language representations. The semantic representation thus feeds back activation and thereby activates the lexical and sub-lexical levels of the corresponding sign representation. In addition, the co-activation of the phonological elements of the sign translation also activates its phonological neighbors; which in turn, produces a conflict in access of the correct lexical item. This explanation therefore assumes top-down feedback activation by the semantic/conceptual level. Figure 2.4 shows an illustration of the operating steps during cross-modal cross-language activation, as proposed by Ormel et al. (2012). 


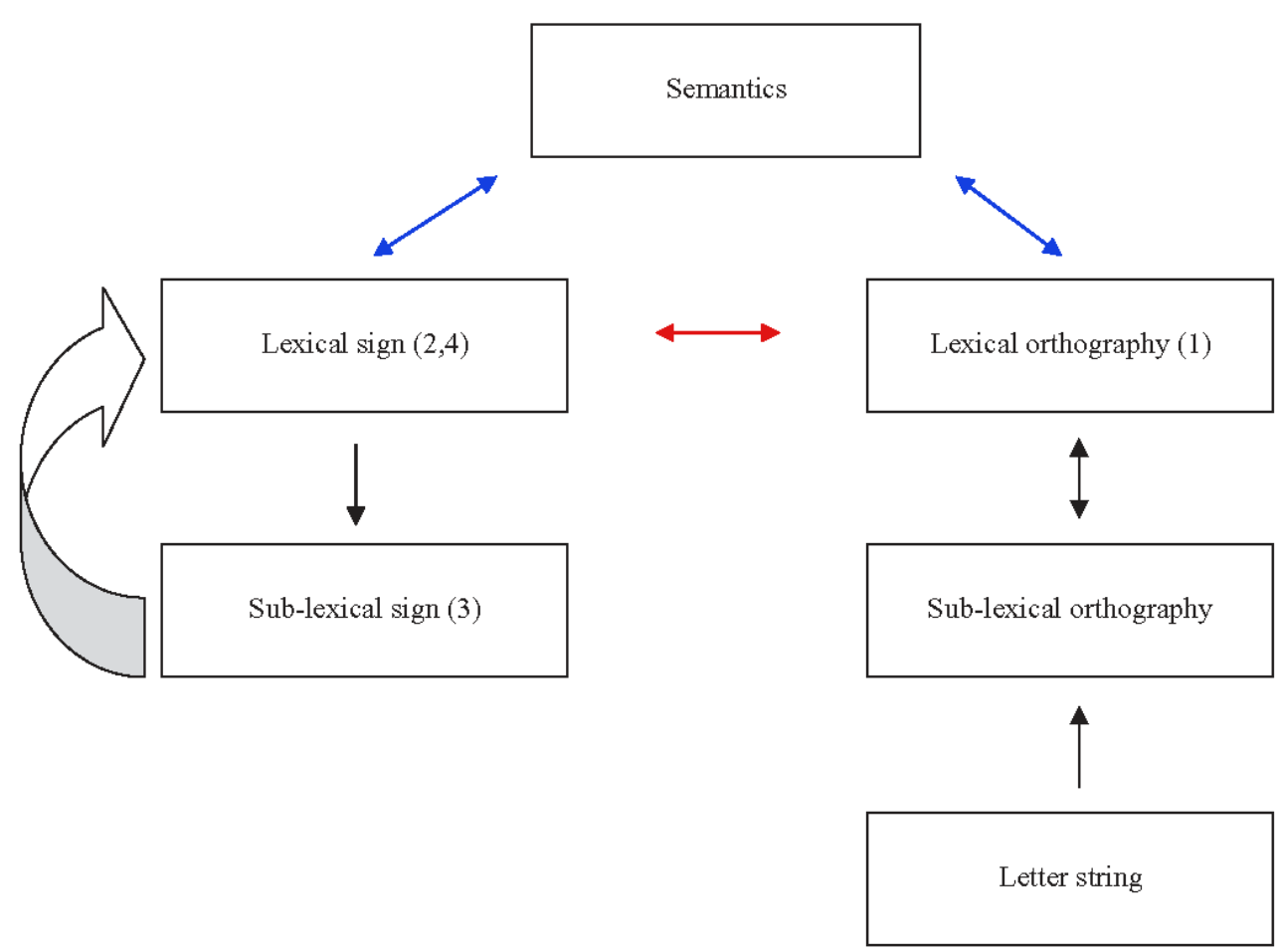

Figure 2.4 Schematic illustration of co-activation pathways as proposed by Ormel et al. (2012): Deaf Bilingual Interactive Activation model (p. 301). Color highlighting by J.H.

As can be seen in Figure 2.4, a possible link between L2 lexical orthography and the lexical sign can either be mediated via an independent semantic node (marked in blue), or it can be directly linked (marked in red).

Following the semantic explanation, we could explain our findings of L2 word co-activation during L1 sign processing accordingly. Thus, the DGS sign input activated the phonological and lexical representation of the sign, which thus activates its semantic concept and by that the phonological/orthographic representation of the German word. Hence, when prime and target were minimal pairs in their German translation equivalent, orthographic/ phonological parts of the German translation of the prime were still activated during the co-activation of orthographic/phonological parts of the German translation of the target. This resulted in lower processing costs of the target and was thus reflected in a less negative polarity of the electrophysiological 
signal. The observed cross-modal cross-language activation in our German related prime-target pairs would therefore be mediated by the shared semantic node of sign and word representations. Since we deliberately controlled our stimulus material in matters of mouthing on prime and target signs (i.e. neither prime nor target sign were accompanied by mouthing), we can exclude direct co-activation via an overt visual cue of the corresponding spoken words. However, although a direct co-activation via the visual cue of the mouthing can be ruled out by the controlled stimuli, we cannot exclude the possibility of an indirect co-activation of the corresponding mouthing. As we will demonstrate in the upcoming paragraphs, an indirect co-activation of the mouthing of a corresponding manual sign can also explain the crosslanguage co-activation effect we found.

However, before we come to that, we will discuss the second explanation of cross-modal co-activation, which assumes a direct link between lexical sign and word representations without the mediation via a semantic node. In a visual world eye-tracking study, Shook \& Marian (2012) demonstrate crossmodal co-activation in hearing bimodal bilinguals, i.e. CODAs. Compared to unrelated distractor pictures, participants' duration and proportion of eyegaze remained significantly longer on competitor pictures that were phonologically related with the ASL translation of the target word. Thus, Shook \& Marian (2012) conclude that participants co-activated the sign translation of the spoken English target word, and thereby also co-activated phonologically related signs like the sign translation of the competitor picture. Since hearing CODAs grow up with switching between both language modalities on every day basis, they have high experience in processing and translating between sign and spoken language, which might result in a direct associative link between lexical items of signs and words. Following this "asemantic link explanation", cross-modal co-activation in hearing CODAs could be explained as follows: An incoming sound stream activates phonological and lexical representations of a word, and via a direct associative link co-activates the corresponding (lexical) representation of the sign. This again activates the phonological representations of the sign and of 
phonologically similar signs. Figure 2.5 shows an illustration of the operating steps during cross-modal cross-language activation, as proposed by Shook \& Marian (2012).

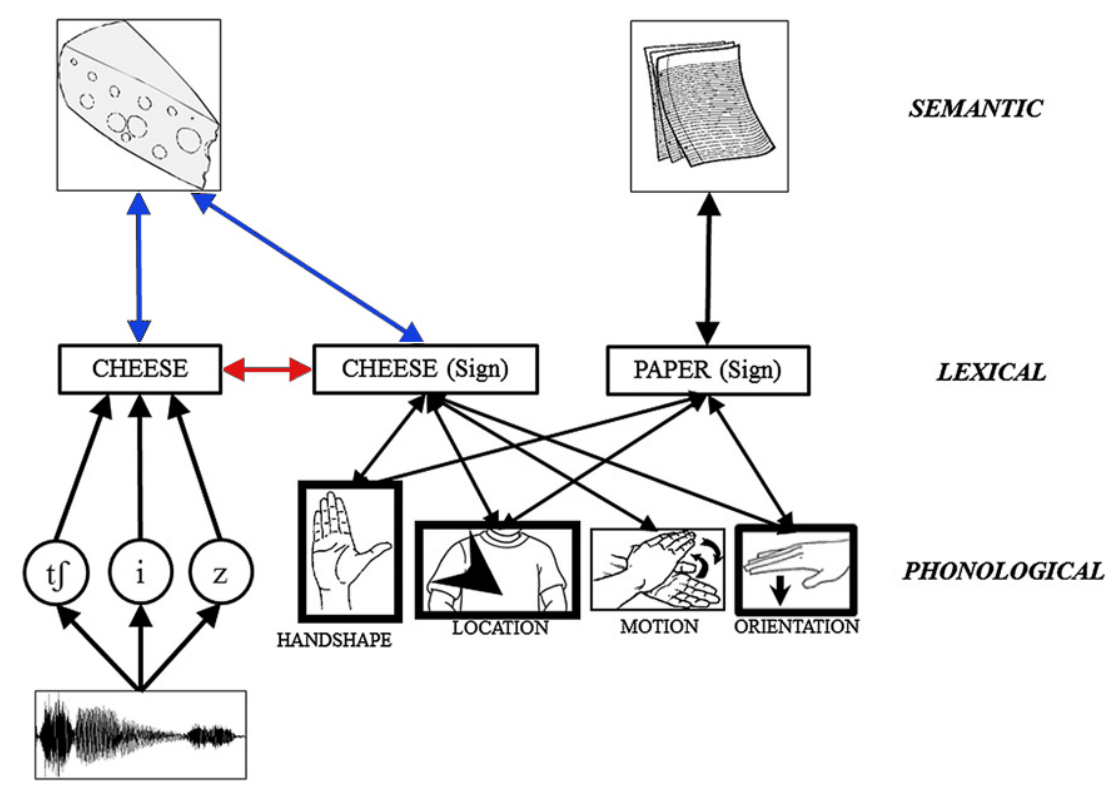

Figure 2.5 Schematic illustration of co-activation pathways in hearing bimodal bilinguals as proposed by Shook \& Marian (2012). Color highlighting by J.H.

Figure 2.5 also shows both explanation possibilities: (i) via an independent semantic node (marked in blue), and (ii) via a directly linked connection on the lexical level (marked in red). Since Shook \& Marian (2012) investigated a heterogeneous group of CODAs with respect to ASL being the first or secondly acquired language, the activation direction between L1 and L2 remains uncertain for their results. Half of the participant group had deaf parents and considered ASL as their native language (ASL $=\mathrm{L} 1)$, while the other half were highly proficient but late learners of ASL and hence acquired ASL as their second language (ASL $=\mathrm{L} 2)$. It is thus uncertain whether $\mathrm{L} 1$ representations activated L2 representations or vice versa. Kroll \& Stewart (1994) showed in a translation task that lexical links between L1 and L2 representations can be 
variably strong and weak. A lexical association is assumed to be stronger for L2 to L1 items compared to L1 to L2 items. During second language acquisition (beyond early childhood) L2 items are learned on the basis of an existing L1 system and are thus lexically linked to L1 items, which makes this associative direction stronger. Therefore, one could assume a difference in coactivation processes from L1 to L2 versus from L2 to L1. However, Shook \& Marian (2012) could show in a post hoc analysis investigating both groups separately, that this, nonetheless, had no impact on the duration and proportion of participants gaze, so both groups showed the same pattern of results.

Based on their results, neither Shook \& Marian (2012) nor Morford et al. (2011) or Ormel et al. (2012) draw a clear-cut conclusion on the question how sign and word representations are mediated during cross-modal crosslanguage activation: "Whether or not sign phonology [...] actually mediates the retrieval of the meanings of written words (pre-conceptual activation) or is activated after access to the meaning (post-conceptual activation) remains an open question for further investigation" (Ormel et al., 2012: p. 301).

Based on the results of our experiment, we cannot resolve this explanational gap either. Yet, we take a step further and discuss a possible explanation for cross-modal co-activation that needs to be tested in future investigations. We argue that the corresponding mouthing of a sign constitutes a shared representation between the sign and words sub-lexical representations. We assume mouthing - i.e. the silent articulation of (parts of) the spoken word simultaneously occurring to the production of a sign - to be part of the sublexical representation of the sign as well as part of the sub-lexical representation of the spoken word. A fundamental assumption to this is that a sub-lexical representation of a spoken word consists of several types of representations: (a) an auditory component, (b) an articulatory component, and (c) a visual component. The auditory component represents the knowledge of how a lexical item sounds, based on its phonemes. The articulatory component includes the knowledge of how a lexical item is 
produced, including the relevant muscle activity. In addition, the visual component includes the knowledge of how the movements of the mouth look like when the particular lexical item is produced by others. Together these factors form the sub-lexical units that constitute the spoken word representation, respectively.

The fact that sub-lexical representations of words contain visual information, can be seen in the well-known McGurk effect (McGurk \& MacDonald, 1976): When participants were exposed to a video with a person producing the mouth movements of [ga], and they simultaneously heard the sound stream of [ba], participants in fact believed to hear [da]. This shows that the conflicting input between a visual impression of a mouth movement and an auditory signal leads to overriding the sounds being perceived. To explain the McGurk effect, Boersma (2012) argues for a representation model that includes visual cues in terms of a mental representation of the "Sensory Form" and "cue constraints", i.e. "[...] the speaker-listener's knowledge of the relation between phonological features and both auditory and visual cues" (Boersma 2012: 301). Thus, visual cues of spoken words seem to be included in sub-lexical representations of words. We assume this to account for representations of words in both hearing individuals as well as in deaf individuals. As deaf people are able to acquire a spoken language, and therefore have the ability to grasp oral information from visual cues of the mouth and can produce spoken language themselves, they must also have sub-lexical representations of spoken words that contain all three kind of information types. The auditory component is obviously less developed and depends on the age and the degree of hearing loss, and the amount of spoken language input perceived by each individual. This is compensated by a larger visual component, including the knowledge of how the mouth movements look like when a word is articulated. The articulatory component represents the knowledge of muscle activity that is needed to produce the word. Thus, the mouthing of a spoken word is represented by both, the articulatory and the visual component of that word. Additionally, the representation of the mouthing is both part of the sub-lexical representation of a spoken word as 
well as the sub-lexical representation of the corresponding sign. The sublexical representation of a sign consists of (i) an articulatory component on muscle activity that is needed to produce the sign (this includes manual as well as nonmanual components), and (ii) a visual component of how a sign looks like when produced by others. Both types of components include the representation of a mouthing and are represented on the sub-lexical level of a sign. Therefore, the mouthing can be a shared component between sub-lexical representations of signs and sub-lexical representations of spoken words, and can thereby initialize cross-modal co-activation. See Figure 2.6 for a schematic illustration of word representations and sign representations for deaf and hearing people.

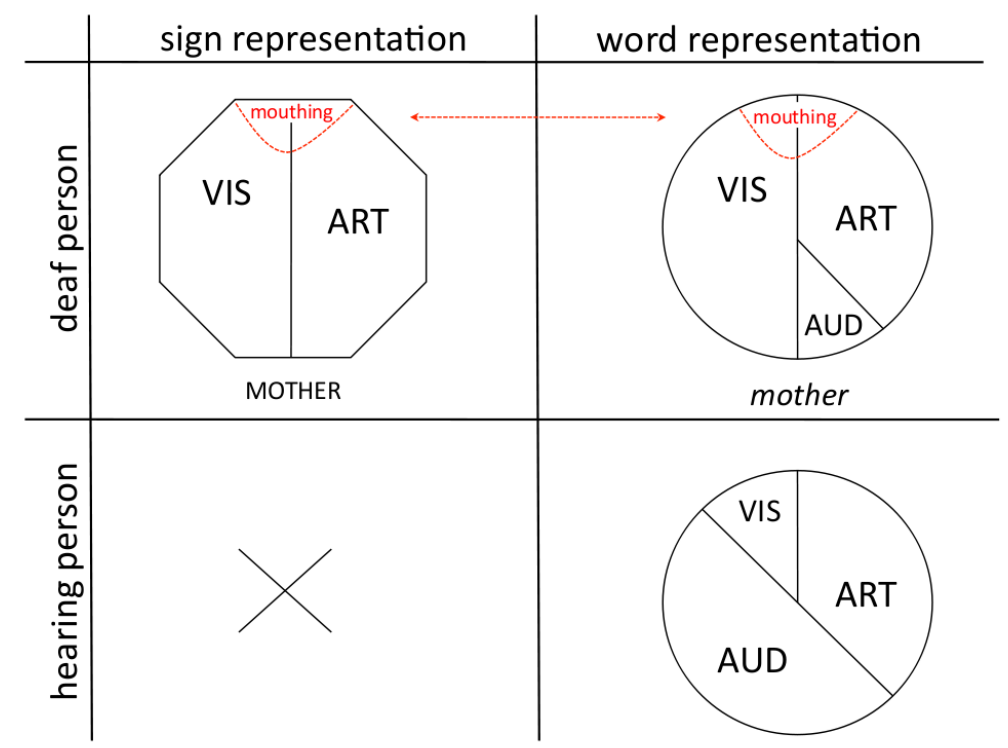

Figure 2.6 Schematic illustration of the sub-lexical representations of a sign and a word (for deaf and hearing people); VIS = visual component, ART = articulatory component, AUD $=$ auditory component. Note that the mouthing is part of the sign representation as well as of the word representation.

Following this assumption, cross-modal co-activation occurs when the sublexical representations of a sign includes the representation of the corresponding mouthing, and through its activation co-activates the sub-lexical representations of the corresponding spoken word that also includes the representation of the corresponding mouthing. 
With this model of a three-partite sub-lexical representation of words including a shared representation of mouthing, we could explain our results as follows: The visual input of the sign (without mouthing) activated the sublexical representation of the sign. Since the mouthing is assumed to be one component of the sub-lexical representation of the sign, it was co-activated. This constituted a link to the sub-lexical representation of the corresponding spoken word and thus co-activated the spoken word lexical representation. The perception of a prime sign like MOTHER activated its sign-phonological units, including its mouthing. This co-activated the sub-lexical units of the corresponding German word representation of Mutter. During the processing of the target sign BUTTER and its co-activated German equivalent Butter, parts of the German word representation of the prime sign were still activated and thus had an impact on the processing costs of the target sign.

Figure 2.7 gives an schematic illustration of the cross-modal cross-language activation explanations: (a) via a semantic mediation as discussed by Morford et al. (2011), Ormel et al. (2012), and Shook \& Marian (2012), (b) via a direct associative link as mentioned by Shook \& Marian (2012); and (c) via mouthing, as we introduce it here. 


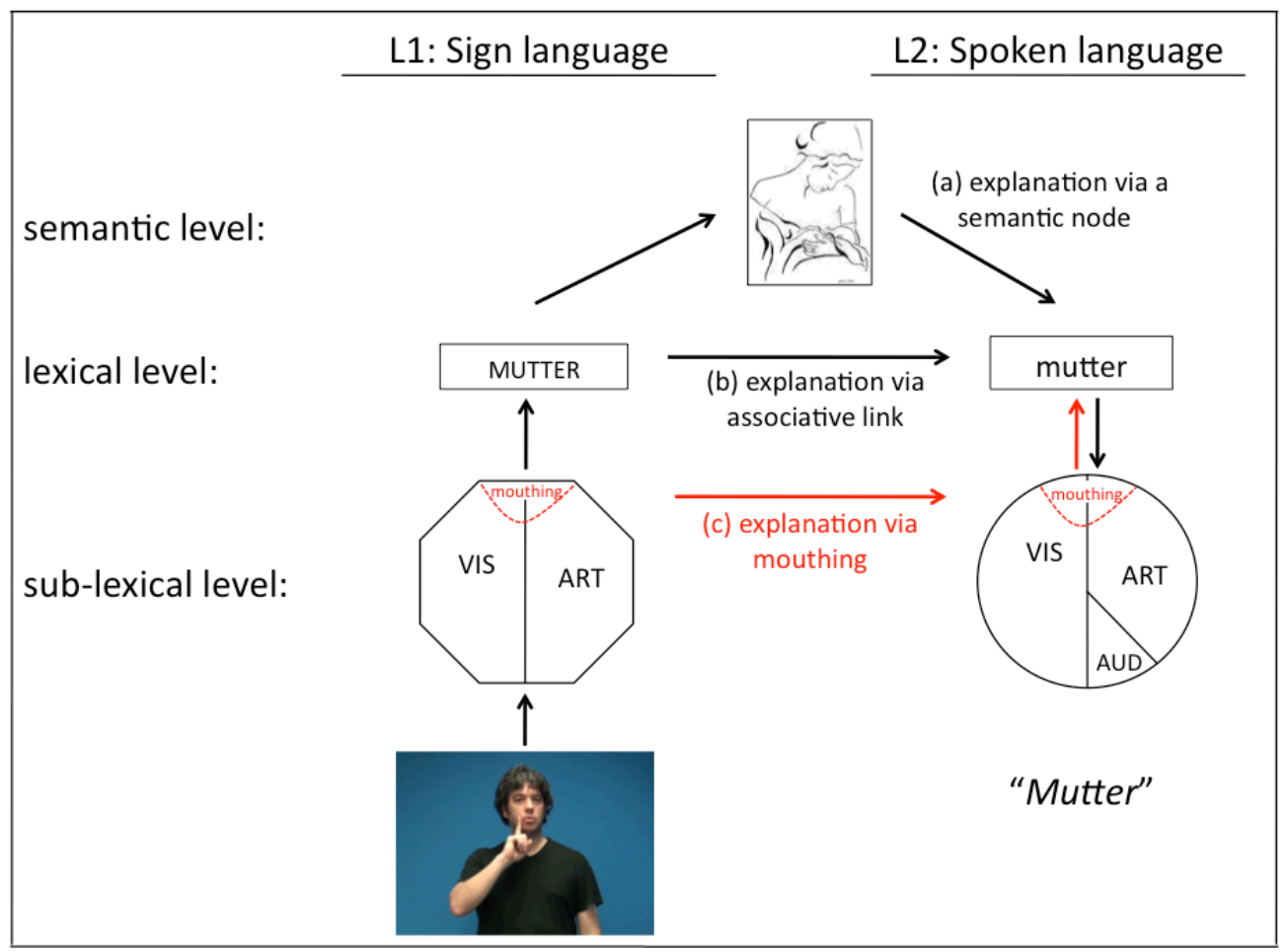

Figure 2.7 Schematic illustration of cross-modal cross-language co-activation. (a) explanation via a semantic mediation, (b) explanation via a direct associative link, and (c) explanation via mouthing.

As becomes apparent from Figure 2.7, the sub-lexical components of the sign directly co-activate the sub-lexical components of the corresponding spoken word, based on the shared representation of the mouthing. Following the idea of a successive lexical activation, this connective link on the sub-lexical level would be more immediate compared to a link on the lexical or the semantic level. Hence, a cross-modal cross-language priming effect based on a sublexical link should then engender a somewhat earlier corresponding ERP effect, prior to $400 \mathrm{~ms}$, which is the approximate peak for the $\mathrm{N} 400$ component in general associated with semantic processing (Kutas \& Hillyard, 1980, Kutas \& Federmeier, 2011). Nonetheless, the effect we observed for cross-language priming in the present study appeared in a 450-650 ms time window. This latency shift could be explained by the fact that our stimulus material was presented without the corresponding mouthings. Thus, the 
mouthing of prime and target signs had to be co-activated separately in order to link the corresponding spoken word representation. This additional activation effort of the mouthing might have caused the latency shift of the priming effect.

Despite the fact that we argue for the plausibility of the "mouthing explanation", we cannot exclude that other links between representational levels modulate the observed cross-language priming effects. For instance, we have not distinguished between priming via orthographic or via phonological minimal pairs. All our German prime-target pairs are orthographic minimal pairs as well as phonological minimal pairs. Due to the limited auditory access to spoken words for deaf individuals, it is difficult to control how well defined the auditory representation of a word is, and whether the orthographic representation is part of a word's sub-lexical representation or not. If the latter would be the case, deaf people would articulate and comprehend in three (instead of two) modalities: in sign language, in spoken language (via lip reading and speaking), and in the written modality (see. e.g., Plaza-Pust \& Weinmeister 2008).

This explanation assumes mouthing to be an integral part of sign representations. However, the linguistic status of mouthing is a highly controversial topic in sign language research: Whereas one side argues that mouthings are part of sign language phonology, because they can have distinctive functions (e.g., the DGS minimal pair WHERE and WHAT differs only in the mouthing "o" for WHERE ('wo') and the mouthing "a" for WHAT ('was')); the other side assumes mouthings to be loan-elements from the surrounding spoken language that have a minor linguistic status in the sign language itself (for an overview, see Boyes Braem \& Sutton-Spence, 2001). For the purpose of our explanation, we here assume a shared sub-lexical representation of mouthing to be the origin of cross-language co-activation. The idea of co-activation via mouthing is supported by a single case investigation of a deaf bilingual women (British Sign Language - English) with left-hemispheric aphasia, named "Maureen" (Marshal et al., 2005). Maureen deafened with 18 months 
and was highly proficient in English conversation, reading, writing and British Sign Language (BSL). Her left-hemispheric cerebrovascular accident led to major constraints in both languages. Hence, she showed no spontaneous language production neither in English nor in BSL, and her comprehension of BSL and English was severely impaired, especially on a semantic level. Nevertheless, in several experimental tasks, Maureen could be cued to produce English words without any semantic mistakes. When presented with a sign plus mouthing (combined cue), Maureen performed best in articulating the corresponding English word. Also, single presentation of mouthing (oral cue) or of a sign without mouthing (sign cue) led to correct production of English words. In contrast, gestural cues (e.g., miming to eat with fork and knife) did not cue Maureen to articulate the word "eat". In addition, Maureen did not succeed in naming objects neither in English nor in BSL. Thus, only the phonological (oral and sign) cues were efficient to trigger English production. Marshal et al. (2005) argue that English word representations and BSL sign representations are directly linked in Maureen's lexicon, instead of being mediated via a semantic node. The authors further assume a direkt link between sign and word representation via mouthing. The rationale for this assumption is that combined cues (sign plus mouthing) were most efficient in eliciting English words, while oral cues alone were rather sufficient to cue English words. Signs alone cued English words by co-activating the corresponding mouthing of the English words. "The above evidence suggests that mouthing should be viewed as a bilingual contact phenomenon, [...]." (Marshall et al., 2005: p. 733).

A further support to this claim is that Maureen could only be cued by 'sign only' cues to produce nouns, not verbs. The explanation is that BSL nouns can be more easily mapped onto English nouns (like BALL and "ball"), compared to BSL verbs that cannot directly map onto English verbs. For example, the English verb "throw" can be articulated with several BSL signs, including morphologically realized classifier constructions of the object and the manner of action. Thus, BSL nouns are more likely accompanied by mouthing than verbs (Sutton-Spence \& Day, 2001). The same seems valid for other sign 
languages like DGS. In our stimulus material, prime-target pairs consisted of nouns only and it is most likely that they would be accompanied by mouthings in a non-experimental conversational context. This strengthens the assumption of a sub-lexical link via a shared representations of the mouthing.

However, the linguistic status of mouthing remains uncertain for a further reason: The amount of use of mouthing can highly diverge between signers and within signing contexts. For example, orally educated elder signers use more mouthing compared to younger signers. Second, deaf people use particularly more mouthing when signing to hearing people compared to signing to other deaf people. And third, contextual aspects like the complexity of a discourse topic influence the use of mouthing. Sutton-Spence \& Day (2001) report that more mouthing is used in information giving contexts compared to narrative contexts. Taken the variability in the usage of mouthing into account, it questions the claim that mouthings are inherently anchored in sub-lexical representations of signs. Further research is needed to test the hypothesis of cross-modal cross-language activation on the basis of a shared mouthing. The linguistic status of mouthing and its impact on sign processing has yet to be determined.

From the neurolinguistic approach, Capek et al. (2008a, 2008b, 2008c) made a remarkable contribution with respect to the underlying cortical distribution and differentiation between speech processing and sign processing in bimodal bilinguals. In an fMRI study, deaf and hearing bimodal bilinguals were presented with (i) videos of silent speech, (ii) videos of solely manual signs with no mouth action, (iii) videos of manual signs with mouthing, and (iv) videos of manual signs with non-speech like mouth action (i.e. echo phonology, Woll 2001). Capek et al. (2008b) investigate whether the cortical correlates of processing sign language differ from the processing of seen speech, and whether the cortical correlates of processing signs with mouthing differ from the processing of signs with mouth actions (both in deaf and hearing bimodal bilinguals). Interestingly, they found distinctive patterns in 
both. Processing seen speech activated the middle and posterior portions of the superior and middle temporal gyri to a greater extend than processing signs, which in turn, elicited a greater activation in the middle and inferior temporal and in the fusiforum gyri. This difference in cortical patterns resembled the pattern differences of processing signs with mouthing versus signs with mouth actions. While the processing of signs with mouthing showed greater activation in the middle and posterior portions of the superior temporal gyrus, the processing of signs with mouth gestures elicited greater activation in posterior portions of the superior, middle and inferior temporo-occipital cortices (Capek et al 2008c: p. 102-105). This indicates that mouthing and seen speech are processed in similar regions distinct from regions for sign and mouth action processing. This supports the assumption of a close link between sub-lexical representations of signs with mouthing and sub-lexical representations of spoken words.

To test the hypothesis of mouthing being the link of cross-modal language coactivation, a first opportunity would be to conduct a corresponding ERP experiment with cross-language priming including prime and target signs articulated with its corresponding mouthing. If sign and word representations are linked on the sub-lexical level, we assume that prime and target signs articulated with mouthing engender a somewhat earlier ERP priming effect prior to $400 \mathrm{~ms}$. Additionally, an ERP experiment using phono-semantic priming could shed light on the semantic link explanation. The German translation equivalent of the prime would hence not be phonologically related to the translation equivalent of the target, but rather to the translation equivalent of a semantic associative of the target. That is, the sign HOUSE ('Haus') would not prime the target sign MOUSE ('Maus', i.e. cross-language phonological priming), but the target sign CAT ('Katze') via its semantic associative mouse (i.e. phono-semantic priming). If cross-modal language coactivation also operates via a semantic link, we would expect an ERP priming effect related to the target sign in a slightly later time window. 
On the basis of behavioral experiments, the impact of mouthing on the processing of signs could be tested with lexical decision tasks or with translation tasks. In both designs, target signs would be presented with and without mouthing in order to detect the amount of ambiguities between signs with and without mouthing. We would expect signs presented without mouthing to be more ambiguous which would be reflected in a higher variety of spoken word translations or in longer reaction times for lexical decision tasks. This would provide more clarity with respect to the discriminating function of mouthings, but could not directly address the question whether mouthings are inherent components of sub-lexical representations of signs.

\subsection{Summary and conclusions}

The present ERP study on German Sign Language priming demonstrates that target signs preceded by phonologically overlapping prime signs can engender a priming effect during sentence processing (within language priming effect). The study also presents evidence for co-activation of second language words during native sign language processing. Target signs preceded by signphonologically unrelated primes but with phonologically related German translation equivalents also engendered a priming effect (cross-modal crosslanguage priming effect). This indicates that signs with an overt phonological overlap can prime each other within sentence processing. Furthermore, deaf bimodal bilinguals also activate orthographic/phonological representations of L2 spoken words during L1 sign language sentence processing. We have discussed several explanations for this cross-modal co-activation based on semantic and asemantic links. Further, we have introduced a third explanation that assumes the mouthing of a sign to be a shared representational component on the sub-lexical level of sign and word representations. This hypothesis needs to be verified by future research. 


\title{
3 Agreement or no agreement. ERP correlates of verb agreement violation in German Sign Language
}

\begin{abstract}
The linguistic status of sign language agreement is a deeply discussed topic. The concordance of a verb and a referent in a corresponding location in the three-dimensional signing space raises the question of an interface between the linguistic system and gestural aspects. However, previous studies investigating agreement violation in sign languages report similar neurophysiological responses to those observed for agreement violation in spoken languages. In this chapter, we present two event-related potential studies (ERP) on agreement violation in German Sign Language sentences. In one study, we investigated the processing of agreement verbs that ended in an unspecified location opposite the location of the associated referent. Incorrect agreement verbs engendered a posterior positivity effect (220-570 ms relative to trigger nonmanual cues) and a left anterior effect (300-600 ms relative to trigger sign onset). These seem to reflect distinct cognitive processes. Crucially, agreement violation with agreement verbs did not elicit a LAN followed by a P600. In a second study, we therefore investigated a violation of morphologically specified plain verbs. Plain verbs that are not specified to mark agreement were articulated in a manner to express third person object agreement. These incorrect forms of "agreeing" plain verbs engendered a broadly distributed positivity effect (420-730 ms post mismatch onset). Both results, for incorrect agreement verbs and incorrect plain verbs, are discussed under the perspective of a violation of well-formedness and under the perspective of enhanced costs for the signer to update his/her situation model. We argue that agreement in sign languages is a modality-specific phenomenon that is rather based on pragmatic than morpho-syntactic principles.
\end{abstract}

\subsection{Introduction}

Agreement in sign languages is not the same phenomenon as agreement in spoken languages. Agreement in spoken languages is the realization of grammatical features of one linguistic element on another (c.f. Barlow \& Ferguson, 1988), for example, the grammatical features of a noun on the verb. Most spoken languages realize subject agreement, so, in a German sentence 
like "Ich helfe meiner Oma" ('I help my grandma') the verb agrees with the subject in the features of person, number, tense, mode, and genus verbi. In all sign languages investigated up to now, we can find a similar phenomenon that has likewise been called "agreement": The sentence above when translated into German Sign Language (DGS) is signed as I MY GRANDMA ${ }_{1} \mathrm{HELP}_{3 a}$ whereas the path movement of the verb HELP begins at the signer's chest and ends at a location that is distinct from the location of the signer and the location of the addressee of the conversation (here labeled with "3a"). In contrast, the verb HELP moves from location $3 a$ towards the location of the signer in the sentence MY GRANDMA ${ }_{3 a}$ HELP $_{1}$ ('my Grandma helps me'). Compare Figure 3.1 for the two forms of HELP.
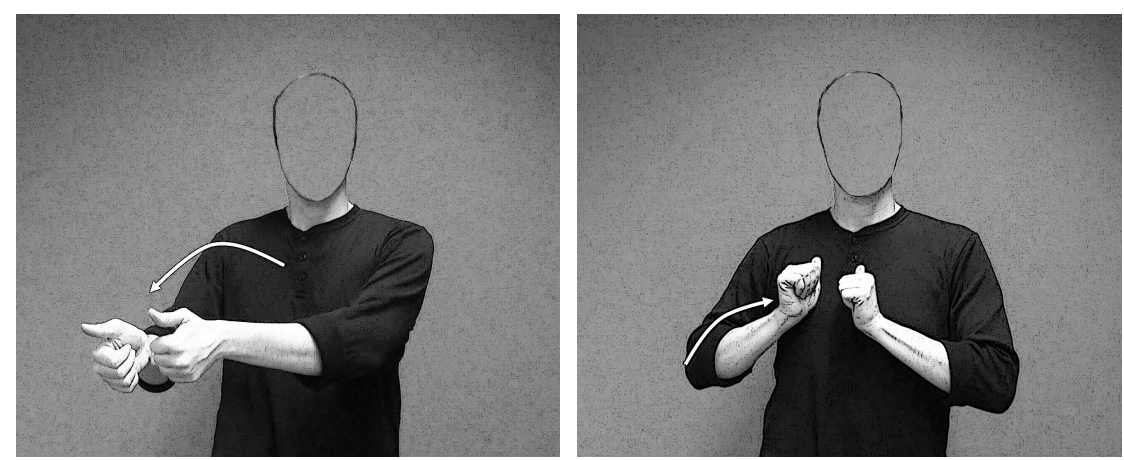

Figure 3.1 Pictures of the sign HELP. Left, from the signer to a $3^{\text {rd }}$ person referent as in ${ }_{1} \mathrm{HELP}_{3 \mathrm{a}}$ ('I help him/her'); and right, from a $3^{\text {rd }}$ person referent to the signer as in ${ }_{3 a} H_{E L P}$ ('he/she helps me').

This phenomenon has been described for all sign languages investigated so far (for an overview, see Mathur \& Rathmann, 2012). Nevertheless, the linguistic status of this phenomenon is not as clear as it might seem at first glance. On the one hand, agreement in sign languages also seems to mark the grammatical features person and number (Rathmann \& Marthur, 2002, 2008; Lillo-Martin \& Meier, 2011) and thus describes modality independent grammatical functions. In this sense, it is analogous to spoken language agreement and must be considered as a core linguistic phenomenon. On the other hand, 
sign language agreement exhibits some modality-specific characteristics that clearly distinct it from spoken language agreement. For example, only a subset of sign language verbs, in fact, inflect for person and number, while plain verbs, in contrast, are not specified to mark agreement. This clearly differentiates from the phenomenon of spoken language agreement and raises the question about its linguistic status. On this premise, some researchers highlight the evitable non-linguistic characteristics and thus claim that the phenomenon is gestural in nature (Liddell 1995, 2000).14

Neurolinguistic investigations of agreement violation in sign languages have not yet resolved this controversy. In an ERP study, Capek et al. (2009) investigated agreement violation with agreement verbs in American Sign Language (ASL) sentences. Similar to spoken language agreement violation, in sentences such as "He mow* the lawn" (Coulson et al., 1998), they report a biphasic ERP effect (LAN followed by a P600) to incorrectly inflected verbs compared to grammatically correct verbs. Hänel-Faulhaber et al. (2014) report a similar biphasic pattern for sentences with incorrect verb agreement in DGS. Both studies argue for agreement in sign languages to be a morphosyntactic process.

However, we present two event related potential (ERP) studies where we tested agreement violation with agreement verbs and with plain verbs and found distinct ERP responses to the typically observed biphasic pattern of LAN and P600. In Experiment A, we investigated the online processing of agreement violation in sentences with agreement verbs (similar to Capek et al., 2009 and Hänel-Faulhaber et al., 2014). Furthermore, in Experiment B, we investigated the online processing of agreement violation with plain verbs. Plain verbs are lexically specified and do not inflect for person and number.

\footnotetext{
14 Several terms have been used to label the above-described phenomenon in sign languages. To highlight the modality different aspects of it, the term "directionality" has been commonly used. This points to the fact that verbs (and also pronouns) change their directionality in order to refer to distinct referents located in signing space. The term "agreement" emphasizes the modality-comprising aspect that agreement in both language modalities marks person and number of the verb's arguments. We will use the terms "directionality" and "agreement" (in sign languages) synonymously, depending on which aspect we want to emphasize.
} 
Thus, we transferred the "agreement principle" to this verb category in order to detect an unequivocal agreement violation. Based on our divergent ERP results, we argue that - despite the structural similarities between sign and spoken language agreement - sign language agreement displays a linguistic but somehow different phenomenon than what is called agreement in spoken languages.

In Section 3.1.1 we review the phenomenon called "directionality" in sign languages and highlight the modality-independent properties that are shared between spoken and sign language agreement. The modality-specific properties of sign language agreement will be outlined in Section 3.1.2. Section 3.1.3 reviews previous electrophysiological studies with sign language agreement. Section 3.1.4 describes the design of the current study on agreement violation with agreement verbs (Methods, Results and Discussion in Section 3.2) and on agreement violation with plain verbs (Methods, Results and Discussion in Section 3.3). In Section 3.4 we provide a general discussion of the interpretation of our results, before we conclude in Section 3.5.

\subsubsection{Modality-independent properties of sign language agreement}

The phenomenon illustrated in Figure 3.1 describes the fact that the directionality of a verb's path movement is based on the locations in signing space that are associated with its arguments, i.e. subject and direct object (or indirect object for ditransitive verbs). The verbs initial hold is at the location associated with the subject, the verb then moves towards its final hold at the location associated with its object. This phenomenon has been well described for many sign languages: American Sign Language (Padden, 1983), Israeli Sign Language (Meir, 1998), British Sign Language (Sutton-Spence \& Woll, 1999), German Sign Language (Rathmann, 2000), Sign Language of the Netherlands (Zwitserlood \& Van Gijn, 2006), Australian Sign Language (Johnston \& Schembri, 2007) (see Mathur \& Rathmann, 2012, for references). 
Sign language agreement (i.e. directionality) is not only restricted to verbs, but also occurs in pronominalization, a phenomenon related to verb agreement (Pfau \& Steinbach, 2006; Steinbach, 2011). Pronouns are realized as INDEX signs (also called "pointing" signs because they grammaticalized from gestural pointing, Pfau \& Steinbach, 2006) and direct towards the real or the associated location of the corresponding referent. Thus, they also agree with their antecedent via an overlap in location. ${ }^{15}$

In order to agree with its arguments, a verb undergoes a phonological change based on morpho-syntactic processes to realize a change in meaning. The phonological changes to express directionality are not only restricted to a change in path movement, as in the DGS verb GIVE or HELP. It can also be conveyed by both, a change of path movement and a change of palm orientation directed towards the object location (DGS CRITIQUE), or only by the change of palm orientation (DGS EXPLAIN). In Figure 3.2 all three kinds of phonological changes are illustrated. Note that these verbs also vary with respect to the iconicity of the transfer that is expressed: While the transfer (of an object) in GIVE is concrete, it is more abstract in the verb EXPLAIN and nonexistent in the verb CRITIQUE.

\footnotetext{
15 Further phenomena are associated with sign language agreement, like classifier agreement and role shift. In classifier agreement, the handshape of the classifying verb changes according to semantic features of the noun class of the object: For example, the Chandshape for flat thick objects as in $\mathrm{BOOK}_{\mathrm{i}}{ }_{1} \mathrm{GIVE}_{2}-\mathrm{CL}: \mathrm{C}_{\mathrm{i}}$ ('I give you a book') versus the F-handshape for thin long objects as in FLOWER $_{k} 1_{1} G I V E_{2}-C L: F_{k}$ ('I give you a flower') (Supalla, 1986; Glück \& Pfau, 1998; Zwitserlood, 2012). Furthermore, in role shift (i.e. a strategy to report utterances in sign languages), person agreement is expressed nonmanually by head and body shift and eye gaze change directed towards the location of the addressee of the quoted utterance (Padden, 1986; Herrmann \& Steinbach, 2007, 2012; for an overview, see Lillo-Martin, 2012). Although these kinds of agreement are related to verb directionality, we will not go into greater detail for the purpose of the present studies.
} 


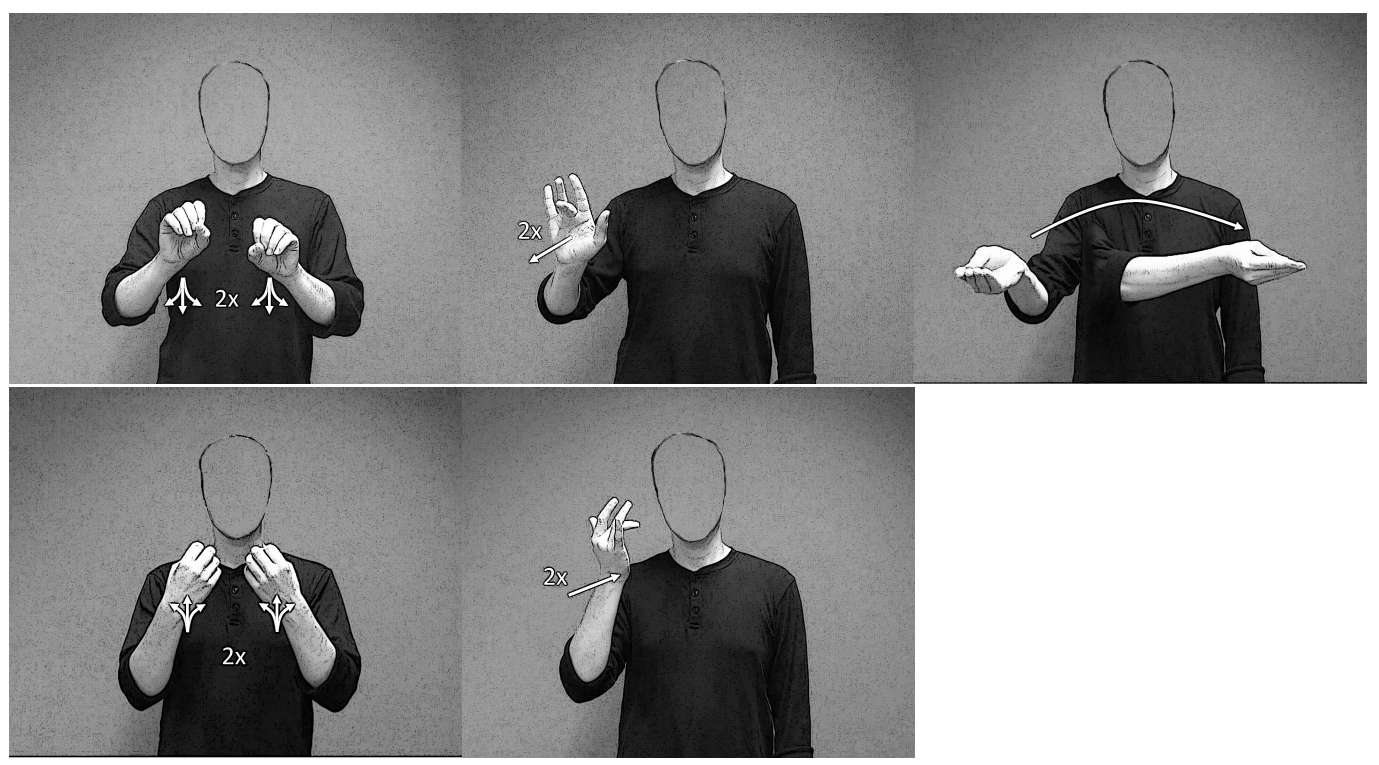

Figure 3.2 Pictures of the verbs EXPLAIN (left, orientation change only), CRITIQUE (middle; movement and orientation change), and GIVE (upper right, movement change only). The verb ${ }_{1}$ EXPLAIN $_{2}$ depicts $1^{\text {st }}$ person subject and $2^{\text {nd }}$ person object (top) and vice versa (bottom). The verb ${ }_{1}$ CRITIQUE $_{3 a}$ depicts $1^{\text {st }}$ person subject and $3^{\text {rd }}$ person object (top) and vice versa (bottom); заGIVE $_{3 \mathrm{~b}}$ depicts two $3^{\text {rd }}$ person referents.

It has been further discussed that agreement with the object is not only expressed manually, but also nonmanually by eye gaze change. Neidle et al (2000) claim that eye gaze towards the location of the object marks object agreement, while a head tilt towards the location of the subject marks subject agreement, both in agreement verbs as well as in plain verbs (which express no overt manual agreement). Thompson et al, $(2006,2009)$ tested this hypothesis for ASL and found a significant distribution of eye gaze towards the location of the object in agreement verbs and towards the location of the locative argument in spatial verbs, but not for plain verbs. Thus, they conclude that eye gaze can co-occur with object and locative agreement like one part of a circumfix. However, eye gaze agreement is not an independent feature checking mechanism as proposed by Neidle et al. (2000). Hosemann (2011) presents a mini-study on eye gaze distribution with all three verb types in DGS. Like in Thompson et al. (2006), there was no eye gaze found with plain verbs that directed towards the object. The study found that spatial 
verbs and agreement verbs were partly accompanied by eye gaze towards the respective argument.

Even though, on the surface, sign language directionality appears very different from concatenational agreement marking in spoken languages, there are universal properties shared by both systems. Assuming an abstract definition of agreement marking as proposed by Barlow \& Ferguson (1988) or by Corbett (2006), agreement in spoken languages and agreement in sign languages are in principle the same linguistic phenomenon. Barlow \& Ferguson (1988: p. 1), define agreement as "a grammatical element X matches a grammatical element $\mathrm{Y}$ in property $\mathrm{Z}$ within some grammatical configuration"; while Corbett's (2006) defining criteria of agreement are "the sharing of features between a 'controller' and a 'target'” within a certain domain (cited from Lillo-Martin \& Meier, 2011: p. 108, see also Marthur \& Rathmann 2012, for references). Thus, in a featural analysis of sign language agreement, as proposed by Rathmann \& Marthur $(2002,2008)$, the arguments of the agreement verb, subject and/or object (i.e. the 'controller'), define the features marked on the verb (i.e. the 'target'). The morpho-syntactic features are person (1st/non-1st) and number (sg./pl.). So, agreement verbs inflect for first person by beginning or ending at the location of the signer (on or near the chest). The inflection for non-first person is realized as a zero form, and hence, a location somewhere in the signing space. Further, agreement verbs inflect for singular (zero marking) or plural (marked with a horizontal arc).

An alternative morpho-syntactic analysis of sign language agreement is in terms of R-loci (Lillo-Martin \& Klima, 1990; Aronoff, Sandler, \& Meier, 2005; Lillo-Martin \& Meier, 2011). In differentiating between conceptual "referential indexes" (R-indexes) and concrete "referential loci" (R-loci), agreement in sign languages between a noun phrase and a verb is sharing a referential index that is overtly realized in a referential loci. Referential indexes are variables associated with each noun phrase that get their value within a discourse, in order to distinguish one referent from another. R-loci, however, are concrete locations in signing space that realize R-indexes. In this sense, 
agreement is a linguistic phenomenon that interacts on a phonological level with gestural space. The interaction between sign language agreement and gestural space is a controversially discussed topic within sign language researchers. A disparate position is represented by Liddell $(1990,1995,2000$, 2003) who argues that the realization of a theoretically infinite number of potential locations in signing space cannot be stored in the mental lexicon, neither as phonemes, nor as morphemes. Thus, directionality cannot be a linguistic agreement process, but has to be gestural in nature. The issue addressed here is called the "listability problem" and raises opposing standpoints (c.f., Wilbur ,2013). For the purpose of the current studies, we do not assume that there are an infinite number of potential loci in signing space (neither in actual usage, nor hypothetically). The listability issue seems to be an artificial problem that does not occur in practical sign language usage. Similar to the ability of categorical discrimination of sounds by hearing people, signers must have the ability of categorical perception of loci in signing space. With regard to the main aim of a conversation, namely that the message will be understood, Wilbur (2013: p. 227) points out that the number of referential loci that is used during a conversation "is generally not above four".

Nonetheless, the usage of R-loci to express person or location reference is unique to sign languages and raises the question whether there are two or three grammatical person categories: first person and non-first person, or first, second and third person (i.e. the signer, the addressee, and a nonaddressed referent). For the relevance of our first experiment on agreement verbs that either correctly agreed with a 3rd person object or ended incorrectly in an unspecified R-locus that was not assigned with a referent, we discuss the topic of the grammatical category person in further detail.

As Meier (1990) and Rathmann \& Marthur (2002) point out, first person can be clearly differentiated from any other person category, because it is realized on or near the signer. In contrast, there is no clear-cut criterion to distinguish between second and third person, which leads to the conclusion that there is 
only a non-first person category. An often-considered criterion to differentiate between second and third person is eye gaze towards the addressee versus eye gaze towards the location of the non-addressed referent. Lillo-Martin \& Meier (2011) showed in a mini-study that eye gaze as a distinctive criterion is not sufficient enough. This study investigated eye gaze during (i) pointing to oneself, (ii) pointing towards the addressee, and (iii) pointing towards a nonaddressed referent. They present percentages of gaze towards the addressee, towards the non-addressed referent, or towards an "other" location during pointing. The gaze towards the addressee during a pointing towards the addressee occurred in only $67 \%$ of the cases, while in the remaining $33 \%$ people gazed towards other directions. This is the same as during pointing in the direction of the third person (63\% gaze towards addressee) and during pointing in the direction to oneself (60\% gaze towards addressee). Further, they found only $31 \%$ of the gaze directed towards the third person during the pointing towards the third person (see Lillo-Martin \& Meier 2011: p. 103 for a tabular overview of the data). Based on this finding, they conclude that eye gaze is neither necessary nor sufficient to be a distinctive factor between second and third person.

However, the distribution of gaze in Lillo-Martin \& Meier's mini-study does not exclude a tripartite person category. Wilbur (2013) points out that although the distribution of eye gaze towards the addressee does not differ significantly across the three pointing directions (self, addressee, nonaddressed referent), the distribution of eye gaze towards the non-addressed referent does differ significantly from the other referents. Basically, signers gazed in two-thirds of the occurrences towards the addressee (irrespective of the pointing direction). Nevertheless, they only gazed towards a third person location during third person pointing (31\%) and not during first or second person pointing (0.06\% and 0.00\%). Therefore, Wilbur (2013: p. 229-230) indicates that gaze towards "addressee" and towards "non-addressed referent" is far from being random. The constraints seem to be: During pointing towards the addressee, gaze towards a non-addressed referent is restricted, and during pointing towards a non-addressed referent, gaze 
towards an 'other' direction is restricted (so gaze must be directed either at the addressee or at the non-addressed referent). Quer (2011: p. 191) gets it to the point that "the linguistic system still must distinguish between [+addressee, -signer] and [-addressee, -signer] person categories by identifying a discrete locus for the addressee in other parts of the grammar (e.g. in imperative forms)." We therefore understand that the loci for the person category [-addressee/+signer] is defined on or near the chest of the signer, the loci for [+addressee/-signer] is defined as the location directed towards the addressee relative to the signer, and the loci for [-addressee/-signer] is defined as a distinct location in signing space that is not the signer location or the addressee location. ${ }^{16}$

In the sense of marking person and number features of subject and/or object on the verb, agreement constitutes a universal language principle independent of language modality. However, there are several observations that distinguish agreement in sign languages from agreement in spoken languages and that are based on the modality specific property of sign language production in a three-dimensional signing space. For example, agreement systems in sign languages do not differ as much as agreement systems in spoken languages, the so-called "uniformity phenomenon". Furthermore, all sign languages investigated so far, show a tripartition of verbs that differ with respect to what they "agree" with: plain verbs show no agreement, spatial verbs agree with locative arguments in a topographical use of signing space, and agreement verbs agree with the object (single agreement) or with subject and object (double agreement) that are marked for [+animate]. In the following section we will discuss the modality specific aspects of directionality that lead to the design of the presented experiments on agreement violation with agreement verbs and agreement violation with plain verbs.

16 To be consistent in labeling, we use a subscript " 1 " for the location of the signer, subscript "2" for the location of the addressee, and subscript " 3 " for the location of a third person. Loci for third person referents can be further differentiated between "3a" on the ipsilateral side of the signer and " $3 \mathrm{~b}$ " on the contralateral side of the signer. 


\subsubsection{Modality-specific properties of sign language agreement}

There are two main aspects that separate agreement in sign languages from agreement in spoken languages: First, agreement in sign languages is restricted to a subset of verbs, and second, the expression of agreement seems to interface with a gestural use of space. The concrete phonological realization of a R-loci (as in verb agreement or in pronominalization) is influenced by the physical location of a present referent. This leads to the question of the gestural impact on sign language agreement and the ambiguity of loci in signing space. In our experiment on agreement violation with agreement verbs (see Section 3.1.4), we contrasted sentences with correct verb agreement with those entailing incorrect verb agreement. Incorrect verb agreement was expressed by an agreement verb ending in locus $3 \mathrm{~b}$ (left side of the signer), whereas the corresponding referent was in fact associated with locus $3 \mathrm{a}$ (right side of the signer). Locus $3 \mathrm{~b}$ was not associated with a referent, and we assume that this locus is unambiguously distinct from locus 3a. We will therefore address both modality-specific aspects of sign language agreement here in more detail.

\section{The tripartition of sign language verbs}

The sign language unique phenomenon of a tripartition of verbs has been confirmed for most documented sign languages so far (see Mathur \& Rathmann 2012, for references). A distinction between plain, spatial and agreement verbs is based on their specification for directionality: Plain verbs constitute a unique verb class because they are lexically specified and cannot undergo a phonological change of their movement path. For example, the transitive DGS verb LIKE has a lexically specified downward movement on the signers' chest. Plain verbs do not necessarily have to be body-anchored, as there are plain verbs articulated on the non-dominant hand or in neutral 
signing space (DGS BUY and PLAY). See Figure 3.3 for pictures of these plain verbs.

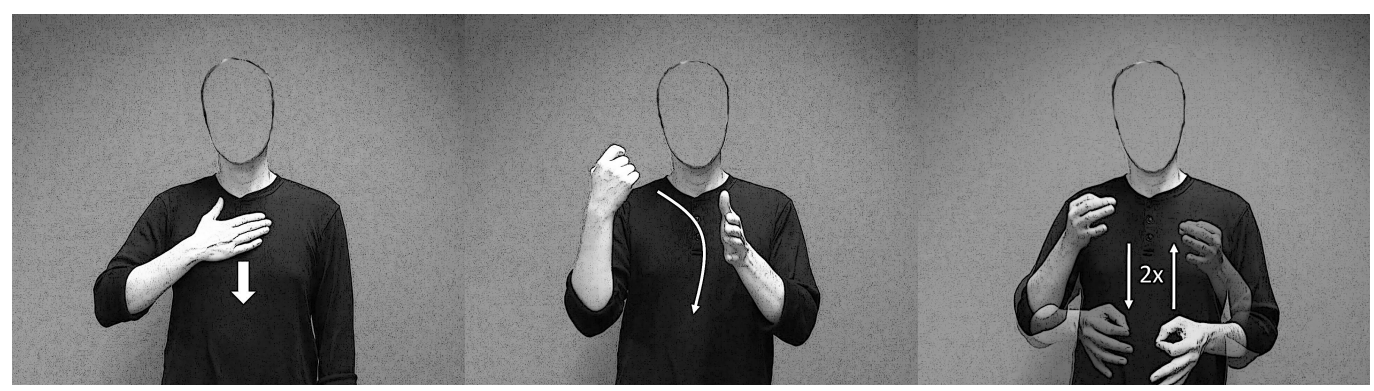

Figure 3.3 Pictures of the DGS plain verbs LIKE, BUY, and PLAY (from left to right) that display a lexically specified movement.

In contrast to plain verbs, agreement verbs have an underspecified path movement that is specified within the discourse in order to agree with the subject and/or the object. While the manner of the movement is specified (as circular, straight or arc movement, etc.), the initial and the final hold of the verb are determined by the referential indexes, manifested in the R-loci, of the discourse referents. Thus, agreement verbs move from the location of the subject towards the location of the object (double agreement) or from a lexically specified location towards the location of the object (single agreement). ${ }^{17}$

Since only a subset of verbs is specified to mark agreement, it is a question as to what criteria a verb is categorized into one or the other verb class. The lexical approach by Padden (1983) assumes a corresponding entry in the mental lexicon. Hence, the lexical entry for a verb contains the information whether it is a plain, spatial or agreement verb. Although a change of status (e.g., from plain verb to agreement verb) could be explained by a change in the lexical entry, this just postpones the explanatory need. To what criterion is a verb marked as being a plain, spatial or agreement verb within its lexical

17 Spatial verbs are realized in a topographic use of space and agree with locative arguments. They are prototypically verbs of action and location (LAY, STAND, GO, SIT, BEAT, etc.) and did not occur in the two present studies. For a detailed description on spatial verbs, the reader is referred to Padden (1993) and Sandler \& Lillo-Martin (2006). 
entry? Further explanations assume a semantic or syntactic category that classifies the verb. Based on the observation of backward verbs (e.g., INVITE or PICK-UP in DGS) that move from the location of the object towards the location of the subject, Meir $(1998,2002)$ offers a morphological explanation of verb categories .The path movement of a verb does not mark subject-object agreement, but rather the semantic roles source and goal. Therefore, the direction of a verb goes from the R-locus of the source to the R-locus of the goal, which is realized by the morpheme DIR. Further, the orientation of the hand (i.e. the facing) realizes a case-assigning morpheme and reflects the syntactic categories subject and object. Thus, the hands face towards the location of the object of the verb. Based on this, the verb categories classify depending on the realization of these morphemes. Plain verbs are neither specified for DIR, nor for the case-assigning morpheme. Spatial verbs are specified for DIR but not for the case-assigning morpheme, and agreement verbs are specified for both DIR and the case-assigning morpheme.

Rathmann \& Mathur (2002) provide a further classification analysis based on the semantic specification of [+animate]. They state that only those verbs agree that take two animate arguments, like, for example, agreement verbs. This explains in particular why some verbs can be used with or without directionality, as their ASL example "I TEACH STUDENTS" (with directionality) versus "I TEACH TOPIC" (without directionality). A different approach by Quadros \& Quer (2008) argues for a bipartite classification of verbs: plain verbs and non-plain verbs. Based on findings in LSC (Catalan SL) and Libras (LSB, Brazilian SL), they argue that the agreeing process is in principle the same for spatial and agreeing verbs. There are verbs that can "agree" with nominals as well as with locatives, like the following example from LSC (Quer, 2011: p. 194).

(4) a. BOOK $\mathrm{K}_{\mathrm{x}} \mathrm{UNDERSTAND}_{1}$

'I understand the book' [locative argument, J.H.]

b. 2UNDERSTAND1

'I understand you'

[person object, J.H.] 
The verb UNDERSTAND is a backwards verb in LSC, similar to COPY in DGS. Thus, it moves from the location of the source to the location of the goal and the facing of the verb marks the object. In (a) the verbs initial hold is on the non-dominate hand (i.e. in this case, a buoy of the sign во0к), and the hand moves to the signer's forehead. In contrast, in (b) the verbs initial hold is at locus 2. Their main argument is that there is no clear-cut distinction between spatial and person agreement verbs, because some verbs can agree with both nominal arguments and locative arguments (UNDERSTAND in LSC, SEE and TEACH in ASL, etc.). Hence, the grammatically interesting process is the difference between agreeing and non-agreeing options of sign language verbs.

Following this line of argumentation, we investigate both kinds of agreement violation: Agreement violation with agreement verbs (these verbs are in principle specified to mark agreement, thus, in the mismatch condition of our stimulus, they agree with a wrong R-locus), and agreement violation with plain verbs (these verbs are not specified to mark agreement, thus, in the mismatch condition of our stimulus, they were artificially modified to "agree" with the object). The notion of "incorrect" inflected verbs, e.g., verbs like GIVE that do not move towards the loci of the object but rather towards a wrong loci (e.g., an unspecified loci in opposite direction) implies that several loci in signing space are distinct from each other and are associated with distinct referents.

\section{The ambiguity of loci}

The second modality-specific aspect, that constitutes a difference between sign language and spoken language agreement, is based on the use of a threedimensional signing space. It is assumed that sign language agreement systems have a gestural origin and emerged from pointing gestures (see Pfau \& Steinbach, 2006, 2011, and Steinbach \& Pfau, 2007 on grammaticalization processes in sign languages). The interface with gestural aspects in sign language pronominalization and verb agreement - for example, that the exact 
phonological form of a pronoun is influenced by physical properties (as the actual position or the height of the corresponding referent) - is a widely discussed topic (Liddell, 2000; Rathmann \& Marthur, 2002; Lillo-Martin \& Meier, 2011; Mathur \& Rathmann, 2012, for references). Therefore, Steinbach (2011: p. 210) refers to agreement in sign languages as a "hybrid category" that undergoes grammaticalization processes from a gestural interface into a more syntactical process, for example, by evolving agreement auxiliaries (see Steinbach \& Pfau, 2007 for a detailed analysis of agreement auxiliaries in sign languages).

Crucial for the purpose of the current studies is the potential unambiguity of referential loci. Outside of a specific discourse, a pronoun like he in English or like IX $\mathrm{X}_{3 \mathrm{a}}$ in DGS is per se ambiguous, because it is referentially "empty". It is unclear as to what a pronoun refers to, until it is anaphorically or cataphorically linked to a referent. Once a discourse is set and the referents are introduced, sign languages seem to be less ambiguous with respect to pronoun resolution because discourse referents are associated with distinct R-loci in signing space and pronouns direct towards the respective R-loci. Consider the sentences in (5).

(5) a. Eng.: "The doctor and the scientist play tennis. He likes the game."

b. DGS: DOCTOR IX 3 a SCHIENTIST IX $3 \mathrm{~b}$ TENNIS-PLAY. IX $3 \mathrm{a} / \mathrm{IX}_{3 \mathrm{~b}}$ GAME LIKE.

The pronoun he in the English example is ambiguous as it can take both noun phrases as antecedent (i.e., either the doctor or the scientist likes the game). In contrast, in the DGS sentence, the two referents are assigned to two different R-loci in signing space, locus $3 a$ and locus $3 b$, respectively. The pronoun in the second sentence has an unambiguous phonological form in directing at one of the two loci. By that it unambiguously refers to either of the two referents. In this sense, the link between a specific R-loci and a 
particular referent within a discourse (either in pronominalization or in verb agreement) is less ambiguous than pronouns in spoken languages.

However, sign language pronouns can also be ambiguous despite the fact that they direct towards a specific location. For example, if more than one referent and/or locative argument are associated with a particular R-locus (e.g., 3a) and a pronoun directs at locus $3 \mathrm{a}$, it is unclear to whom or what the pronoun refers to. In example (6), both a noun phrase and a locative argument are associated with a specific R-locus. ${ }^{18}$

(6) a. SCHOOL IX $\mathbf{X}_{\mathbf{3}}$ KIDS BREAK. SAME TIME HOUSE IX $\mathbf{3 b}$ PARENTS COFFEE DRINK. 'The kids at school have a break. At the same time the parents drink coffee at home.'

b. IX $3 \mathbf{3 a}$ LOUD.

'there it is loud' [at school] / 'they are loud' [the kids]

c. IX $\mathbf{X}_{3 \mathbf{b}}$ QUIET.

'there it is quiet' [at home] / 'they are quiet' [the parents] / 'he is quiet' [the father] / 'she is quiet' [the mother]

When two or more potential referents are associated with a specific R-locus, a pronoun directed towards that R-locus is likewise ambiguous. The phonological form of the pronoun (either IX $\mathrm{X}_{3 \mathrm{a}}$ or $\mathrm{IX}_{3 \mathrm{~b}}$ ) does not evidently identify the referent. In that sense, sign language pronouns are equivalent to spoken language pronouns. The ambiguity can only be resolved by the context and not by the phonological form of the pronoun.

A further case of pronoun ambiguity in sign languages is mentioned by Quer (2011), who points out that the one referent to one R-locus ascription is not as straightforward in everyday language use as it is in theoretical terms. Based

18 I would like to thank Josep Quer for pointing out this aspect. (J.H.) 
on observations in LSC (Barberà 2012), one referent can be referred to by the use of several R-loci in a connected discourse. Also, one R-locus can be used to refer to several referents. Thus, although sign language pronouns within a continuous discourse are theoretically phonologically distinct, this clear-cut distinction does not always occur in everyday language use.

In summary, from a theoretical perspective, sign language agreement is a morpho-syntactical process in that the grammatical features person and number of subject and/or object are realized on the verb. The verb's arguments and the verb share a referential index that is realized in a discourse as a referential locus. Only verbs that take two animate arguments are specified for person agreement. In contrast, there is a subset of verbs (i.e. plain verbs) that is lexically specified and cannot mark agreement. The use of loci in space in order to mark agreement is unique to sign languages and it depends on the context whether two distinct loci have an unequivocal reference. In Section 3.1.3, we will review two recent ERP studies on sign language agreement violation, in order to see if the neurolinguistic perspective can shed light on the agreement phenomenon in sign languages.

\subsubsection{Previous electrophysiological studies on sign language agreement violation}

Morpho-syntactic agreement violation in spoken languages has been extensively investigated in electrophysiological studies, ever since Kutas \& Hillyard (1983) (for a selective overview on agreement violation in the written modality, see Molinaro et al., 2011). Many of these studies investigated number agreement violation between subject and verb, as in "The elected officials hopes* to succeed" (Osterhout \& Mobley, 1995), or between pronoun and verb, as in "Every Monday, he mow* the lawn" (Coulson et al., 1998). Agreement mismatch in spoken languages typically evokes a biphasic ERP pattern with a left anterior negativity (LAN) between 300-400 ms and a late positivity (P600/SPS) after $500 \mathrm{~ms}$. 
In sign language research, Capek et al. (2009) conducted an extensive study on the processing of semantic and morpho-syntactic violations in ASL sentences. They presented videos with ASL sentences that were either semantically or mopho-syntactically correct or contained a semantic or a morpho-syntactic violation (for a discussion on their semantic violation condition, see Chapter 1, Section 1.1.3). In their morpho-syntactic violation condition, they investigated two kinds of violation with agreement verbs: first, "reversed verb agreement violation", and second, "unspecified verb agreement violation" (Capek et al., 2009: p. 8785). In reversed verb agreement, the verb moved from object to subject location (instead of vice versa), while in unspecified agreement violation, the verb moved from the position of the subject towards an unspecified locus that was not associated with the object or any other referent. (7) displays the original stimulus example from Capek et al. (2009: p. 8785).

$$
\text { ASL: MY NEW CAR BLACK CL3a PRO1 MUST 1 } \text { WASH }_{3} \text { a EVERY WEEK3a. }
$$

Engl.: 'My new car is black. I have to wash it every week.' ${ }^{19}$

The correct control sentences contained two referents (either two $3^{\text {rd }}$ person referents or one $3^{\text {rd }}$ person referent and the signer) and an agreement verb moving from the R-locus associated with the subject to the R-locus associated with the object. Note that ASL is a subject-verb-object language, so that the critical verb precedes the object sign. Although Capek et al. (2009) is not explicit in reference to this, there are two sentences here: The first sentence, MY NEW CAR BLACK CL3a, establishes the two referents $\left(1^{\text {st }}\right.$ person and $3^{\text {rd }}$ person) and associates them with R-loci in signing space (CL3a assigns the $3^{\text {rd }}$ person referent to the right side of the signer). In the second sentence,

19 Translation by J.H. In the original example, R-loci in signing space were represented with letters: "a" for the signer (here subscript 1), "e" for the right side of the signer (here subscript 3a), and "c" for the left side of the signer (here subscript $3 b$ ). "CL3a" is a classifier construction, locating the antecedent (i.e. "CAR") at the R-locus right to the signer; " $\mathrm{PRO}_{1}$ " is the first person pronoun. 
$\mathrm{PRO}_{1}$ MUST 1 WASH$_{3}$ a EVERY WEEK3a, the critical verb moves either from subject to object in the correct condition $\left(1 \mathrm{WASH}_{3 \mathrm{a}}\right)$, or in a morpho-syntactically inappropriate direction in one of the agreement violation conditions. In the reversed verb agreement violation condition, the verb moves from the R-locus of the object towards the R-locus of the subject (CAR CL3a ... PRO ${ }_{1}$ MUST 3aWASH$_{1}$ ), and in the unspecified verb agreement violation condition, the verb moves from the R-locus of the subject towards an unspecified R-locus opposite to the assigned R-locus (CAR CL3a ... PRO ${ }_{1} \mathrm{MUST}_{\mathbf{1}} \mathbf{W A S H}_{\mathbf{3}}$ ).

Interestingly, Capek et al. (2009) found different ERP results with respect to the two different morpho-syntactic violations. This indicates that the interpretation of "agreement" in sign languages highly depends on the spatial relations between the referents. Both agreement violation conditions elicited an early anterior negativity (140-200 ms in reversed agreement violation and 200-360 ms in unspecified agreement violation) followed by a late posterior positivity (P600) in the time windows 475-1200 ms and 425-1200 ms, respectively. However, Capek and colleagues report that the anterior negativity was largest over the left lateral anterior site in the reversed agreement condition, while it was largest over the right lateral frontal site in the unspecified agreement condition. In order to explain this difference, they point out that there are different demands on the system in processing unspecified agreement violation versus reverse agreement violation. In unspecified agreement violation, the agreement verb refers to an R-locus at which no referent had previously been located. Thus, participants interpreted the verb ending in an unspecified R-locus either as referring to a new referent (that might be introduced later in context) or as referring to the previously introduced referent located at a different R-locus. These seem to be distinct processing procedures compared with reverse agreement violation, in which the verb moves from the location of the object towards the location of the subject (instead of vice versa).

A second study investigating verb agreement violation during sentence processing is the study by Hänel-Faulhaber et al. (2014) on sentences in 
DGS. $^{20}$ Like Capek et al. (2009), they constructed sentences with two referents. However, these were two $3^{\text {rd }}$ person referents (no $1^{\text {st }}$ person included), both overtly assigned with locus $3 a$ (referent $A$ ) and locus $3 b$ (referent B). The critical agreement verb was positioned in the middle of the sentence (followed by a subordinate clause) and moved from subject location to object location in the correct control condition. Since DGS, in contrast to ASL, is a subject-object-verb language, the critical sign is produced after subject and object signs. (8) represents an original example by HänelFaulhaber et al. (2014: p. 7). ${ }^{21}$

DGS: BOY POINT3a GIRL POINT3b 3aNEEDLE3b REASON POINT3b SLOW SWIM.

'The boy needles the girl because she is slowly swimming.'

The verb agreement violation condition in this study crucially differed from the agreement violation conditions in Capek et al. (2009). While Capek et al. investigated reverse agreement verb violation and unspecified agreement verb violation, Hänel-Faulhaber et al. (2014) presented the agreement verb beginning at an unspecified neutral R-locus in front of the signer (here indicated by subscript " $3 c$ ") moving towards the location of the signer: BOY POINT $_{3 a}$ GIRL POINT3b 3cNEEDLE 1 [...]. This is a relevant difference, because neither the initial- nor final-hold of the agreement verb overlap with one of the R-loci associated with subject and object. They presented the sentences sign by sign and measured EEG responses to the critical verb. For the agreement violation condition, they report a negative potential with a left lateralized frontal distribution (LAN) at 400-600 ms and a late positivity with a posterior distribution (P600) at 1000-1300 ms. The latency shift in these effects is derived from the related trigger position. Due to the fact that the

20 Hänel-Faulhaber et al. (2014) also investigated a semantic violation condition and found an N400 effect that is not further mentioned here.

21 POINT is an index sign locating a referent at a specific R-locus. For a coherent labeling of loci in signing space, we changed the original coding of subscript a (left to the signer) and subscript b (right to the signer) into " $3 \mathrm{a}$ " and " $3 \mathrm{~b}$ ", respectively. 
initial location of the target verb is already detectable during the transition phase which precedes the sign onset of the target verb, Hänel-Faulhaber et al. (2014) time-locked the critical window of the ERP analysis to a trigger, placed at the moment of handshape change within the preceding transition phase (cf., Hänel-Faulhaber et al., 2014: p. 7). This is said to cause the latency shift in the ERP response.

Both studies provide clear evidence that semantic and morpho-syntactic aspects in sign languages are processed differently. They report similar findings to those for spoken language semantic and agreement violation, suggesting a processing system that is modality independent (compare Coulson et al. 1998, for example, for morpho-syntactic agreement violation in a spoken language). Although the results indicate that "agreement" in sign languages is a morpho-syntactic phenomenon, the design of the studies shows that the violation of person agreement in sign languages can be conveyed in several "types" that seem to elicit different ERP responses: An agreement verb violation ending in an "empty" $3^{\text {rd }}$ person location elicited a greater anterior negativity on the right hemispheric site compared to a reverse agreement violation or unrelated agreement violation as in HänelFaulhaber et al. (2014).

In the following Section 3.1.4, we outline the design of our two studies on agreement violation: In Experiment A, we tested agreement violation with agreement verbs that end in an unspecified R-locus where no referent has been assigned to (this is comparable with Capek et al.'s "unspecified agreement violation" condition). In a second study, Experiment B, we tested agreement violation with plain verbs. Plain verbs are lexically specified and cannot inflect for subject and/or object. Thus an agreement violation with plain verbs is a clear violation to morpho-syntactic specifications. To our knowledge, there are no studies investigating the case of agreement violation with plain verbs, so far. 


\subsubsection{The two present studies}

Experiment A on agreement violation with agreement verbs had the following objectives: The first aim was to test "unspecified" agreement violation for German Sign Language and to replicate Capek et al.'s (2009) findings. A further aim was to investigate the time course of processing agreement violation in more detail and time-lock ERPs to different information sources available during the ongoing signing stream of the transition phase and the critical sign (compare Chapter 1 on sign processing within transition phases).

We defined agreement violation as incorrect object agreement. Thus, we presented deaf native signers videos of two consecutive sentences: The first sentence introduced the two referents of interest, the $1^{\text {st }}$ person signer and a $3^{\text {rd }}$ person referent, which was clearly assigned to the right side of the signer at locus 3a. This was followed by a second sentence, which continued the discourse topic and ended with the critical agreement verb. In the match condition, the verb agreed with the subject and object and thus moved from the location of the signer (locus 1 ) to the R-locus associated with the $3^{\text {rd }}$ person referent (locus $3 \mathrm{a}$ ). In the mismatch condition, the verb did not correctly inflect for object agreement, and therefore, moved from the location of the signer (locus 1) towards an unspecified R-locus on the left side of the signer (locus 3b). See example (9) for a representative stimulus sentence with corresponding video stills of the final hold of the critical verb (Figure 3.4).

(9) a. Match condition (agreement verbs):

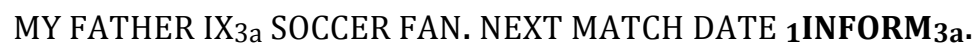

'My father is a soccer fan. I will inform him about the date of the next match.'

b. Mismatch condition (agreement verbs):

MY FATHER IX 3 a SOCCER FAN. NEXT MATCH DATE $\mathbf{1}_{\mathbf{1}}$ INFORM $_{\mathbf{3}}$.

'My father is a soccer fan. I will inform xxx about the date of the next match.' 


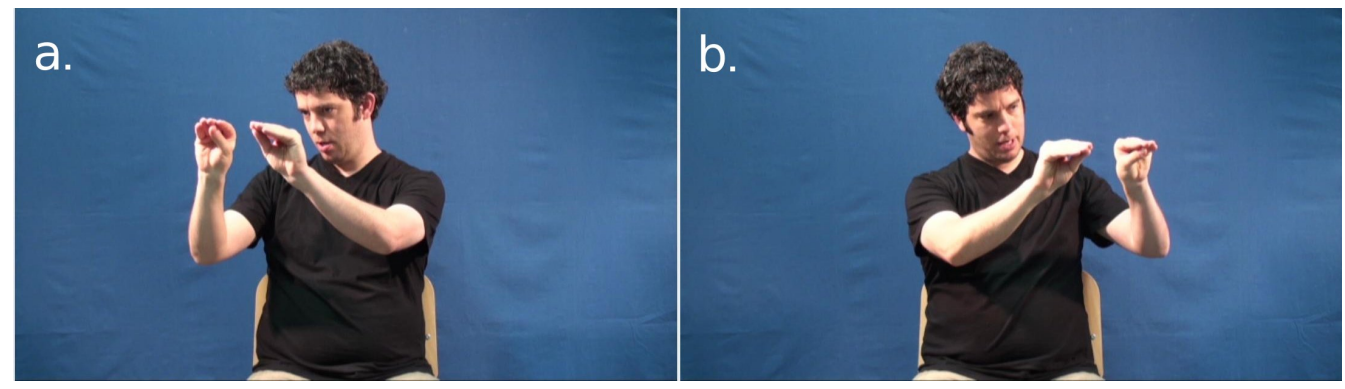

Figure 3.4 Original video stills of the final hold of the critical verb INFORM. (a) in its matching form, agreeing with the $3^{\text {rd }}$ person referent ( INFORM $_{3 a}$ ), and (b) in its mismatching form, ending at an unspecified R-locus on the left side of the signer (1INFORM 3 b).

Note that this kind of violation we tested with agreement verbs, namely "unspecified" agreement violation, is not phonologically incorrect as it can appear in sentences with a referent associated to R-locus $3 \mathrm{~b}$. If sign language agreement indeed constitutes a morpho-syntactic process of marking the features, person and number, of subject and object on the initial and final hold of the verb, we expect a similar ERP response as the well investigated biphasic pattern of LAN and P600 for spoken language agreement violation (c.f., Molinaro et al., 2011). On the contrary, if the phenomenon of directionality in agreement verbs is rather a contextual discourse phenomenon, e.g., a linking process of a previously introduced referent to the subsequent proposition, we would expect to find a different ERP pattern compared to spoken language agreement violation.

In contrast to agreement verbs, plain verbs have a lexically specified initial and final hold. So, they cannot undergo a phonological change in order to mark subject and/or object agreement. However, in Experiment B, we investigated a violation of this non-agreeing specification of plain verbs. We transferred the agreement principle and manipulated plain verbs in the way that their final hold ended at locus $3 \mathrm{a}$, in order to "agree" with a $3^{\text {rd }}$ person referent. Hence, in the match condition, sentences comprised a subject, a $3^{\text {rd }}$ person object, and a sentence final plain verb in its lexical form. However, in the mismatch condition, the plain verbs path movement was extended and directed at the R-locus $3 \mathrm{a}$ associated with the $3^{\text {rd }}$ person referent. Thereby, 
we applied the agreement principle of location overlap between a referent and the final hold of the verb. In any other respect, sentences were identical to their controls. Representative stimulus sentences with a correct plain verb (a) and with an incorrect plain verb (b) are presented in example (10) and the corresponding video stills in Figure 3.5.

(10) a. Match condition (plain verbs):

IX 1 LAPTOP BUY.

'I buy a laptop.'

b. Mismatch condition (plain verbs):

IX $_{1}$ LAPTOP BUY 3 -

'I *buy a laptop.'
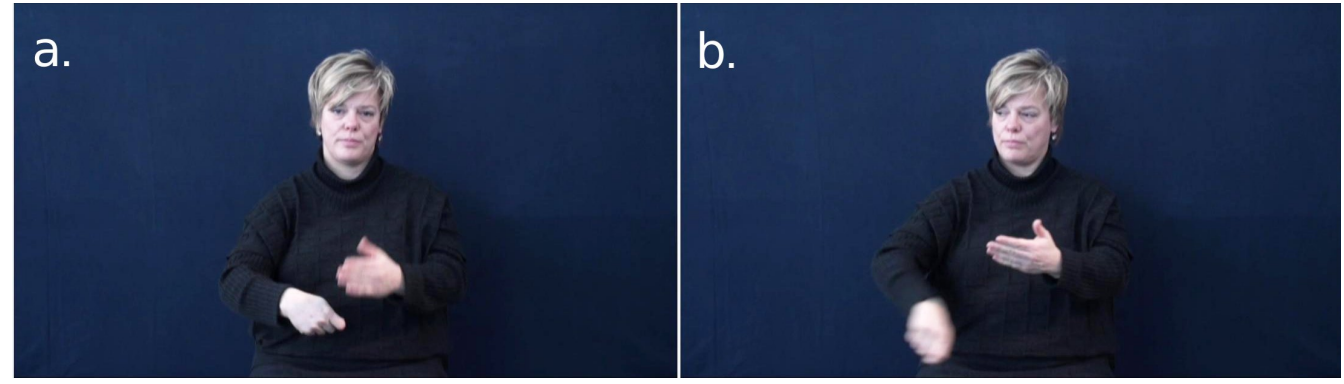

Figure 3.5 Original video stills of the critical plain verb BUY. (a) in its lexical form, as in the sentence IX ${ }_{1}$ LAPTOP BUY, and (b) with the extended path movement directing at locus $3 \mathrm{a}$, in order to mark agreement with the $3^{\text {rd }}$ person object, as in the sentence $\mathrm{IX}_{1}$ LAPTOP BUYЗа.

In contrast to previous ERP studies on agreement violation in sign languages, this experiment is the first to investigate agreement violation with plain verbs. If the artificial path movement towards the object is indeed processed as a form of agreement, we expect a left anterior negativity to reflect the violation of expectancy and a late positivity for some kind of mapping or integrating processes on the sentence level. Regarding neurophysiologic 
responses to agreement violation of non-agreeing verbs, this experiment breaks new ground.

\subsection{Experiment A:}

\section{Agreement violation with agreement verbs}

In Experiment A we investigated agreement violation with agreement verbs, in the form of unspecified agreement: Agreement verbs in the mismatch condition violated the agreement principle as they directed toward the R-locus $3 \mathrm{~b}$ that had not been assigned previously with the subject or object. The experimental set-up is described in the following sections.

\subsubsection{Exp. A: Materials and methods}

\subsubsection{Participants}

A total of 20 congenitally deaf native signers of DGS participated in this experiment as paid volunteers ( 8 female, 12 male) after giving written informed consent. Their ages ranged from 18 to 51 years (mean age: 36 ). All signers had deaf parents or DGS input before the age of three and reported DGS to be their native language. All participants were right-handed, had normal or corrected-to-normal vision and reported no history of neurological disorders. Two participants were excluded from the final data analysis due to excessive eye movement artifacts.

\subsubsection{Materials}

The materials comprised 40 sentences for each of the critical conditions (match versus mismatch) illustrated in example (9) in Section 3.1.4. The 40 sentences per condition were constructed using 10 verbs, thus resulting in 4 repetitions of each individual verb per condition; crucially, sentence contexts 
differed across repetitions. The verbs were frequent agreement verbs, like HELP, ASK, VISIT, or GIVE, and selected for two animate arguments (subject and object). They were checked for grammatical and semantic correctness, frequency and possible dialectical variation by two deaf native informants. Although backwards verbs (like PICK-UP or INVITE in DGS) also take two animate arguments and also agree with subject and object, they were not used as critical verbs in the experiment. The corresponding sentences in both conditions were identical, but for the final critical verb. Critical agreement verbs in the match condition started at the R-locus of the signer (subject) and ended at the R-locus previously associated with the object referent on the right side of the signer (R-locus 3a). In contrast, critical agreement verbs in the mismatch condition also started at the R-locus of the signer (subject), but ended on the left side of the signer at the unassigned R-locus $3 \mathrm{~b}$. Following Thompson et al.'s (2006, 2009) findings of nonmanual object agreement marking with eye gaze, we instructed our informants to mark manual agreement in occurrence with the nonmanual components of eye gaze and head tilt in the direction of the final location (i.e., locus 3a for matching verbs and locus $3 \mathrm{~b}$ for mismatching verbs, respectively). In any other respect, nonmanual action (like facial expressions or brow raise) was kept to a minimum within the possibilities of natural signing. A complete list of stimulus sentences is provided in Appendix A.

The material was discussed, developed and recorded on video together with two deaf DGS informants (one male, one female). Sentences were recorded with a HDR-XR 550E full-HD camera (25 frames/second) and cut and processed with the video editing software application Adobe Premiere Pro. Each video started with $2000 \mathrm{~ms}$ in which the signer remained motionless, before he/she started to sign the sentence in a natural manner. After the end of the sentence, the signer again remained motionless for a further $1500 \mathrm{~ms}$. In total, the videos had an average length of 8.27 seconds (sd 0.66 ). Moreover, in order to ensure maximal naturalness, the videos were not modified in any way (i.e. there was no cross-splicing, no length adaptation etc.). 
The 80 critical sentences resulting from this design (40 per condition) were combined with 80 filler sentences and presented in two different pseudorandomized orders (counterbalanced across participants). The fillers realized a separate experimental manipulation unrelated to the present design. To familiarize participants with the procedure of the experiment, an additional set of 10 sentences with the same structure and a final verb that agreed with the object referent were recorded in the same way as the critical stimuli and presented twice as an exercise before the actual experiment started.

\subsubsection{Procedure}

Experimental sessions were conducted in a dimly lit cabin. Participants were seated in a comfortable chair placed approximately $1 \mathrm{~m}$ in front of a $17 \mathrm{inch}$ computer screen. Each trial began with a video containing a critical sentence and was followed by two tasks: an acceptability judgment and, following $500 \mathrm{~ms}$ of blank screen, an evaluation judgment. The acceptability judgment task (Is the sentence correct? - answers: yes, no) was cued by a question mark in white Arial font (size 60) on a black screen. The subsequent evaluation task (How sure are you? - answers: very sure, sure, not sure, not sure at all) was cued by a short question in German ("wie sicher?", 'how sure?') in the same font. Maximal reaction times for the two tasks were 2000 and $3000 \mathrm{~ms}$, respectively. Following an inter-trial interval of $2500 \mathrm{~ms}$, the presentation of the next video began. Participants were asked to avoid body movement and eye blinks during the video presentation.

At the beginning of the experimental session, all participants watched an introductory video that explained the upcoming procedure. The video was presented in DGS and questions were answered if anything was unclear. All communication between the experimenter and the participants took place in DGS. 
The experimental session began with a short practice session, followed by 4 blocks of 40 sentences each. Between blocks, participants had the opportunity to take short breaks. The experiment lasted for about 45 minutes.

\subsubsection{EEG recording}

EEG data were recorded by means of 32 active electrodes placed according to the international 10-20 system (ActiCAP 32, Easycap GmbH, Herrsching, Germany) amplified using a BrainAmp amplifier (Brain Products $\mathrm{GmbH}$, Gilching, Germany) using a sampling rate of $500 \mathrm{~Hz}$. EEG recordings were referenced to the right mastoid online, and re-referenced to linked mastoids. $\mathrm{AFz}$ served as the ground electrode. Average impedances were kept below $10 \mathrm{k} \Omega$. The electrooculogram (EOG) was monitored for each participant with electrodes at the outer canthi of each eye (horizontal EOG) and above and below the right eye (vertical EOG).

\subsubsection{EEG data preprocessing and statistical analysis}

The raw EEG was preprocessed using a $0.3-20 \mathrm{~Hz}$ band-pass filter, which served to eliminate slow signal drifts. Single subject ERP averages were calculated per condition and electrode from -200 to $1000 \mathrm{~ms}$ relative to four different trigger points (see below). Subsequently, grand averages were computed over all participants. Trials containing EOG or other artifacts were excluded from the averaging procedure by a rejection threshold of $40 \mu \mathrm{V}$.

In order to investigate the processing of verb agreement in a natural sentential environment, trial videos were presented in an unsliced manner. Thus, trigger points for the analysis of the EEG signal were time-locked to different crucial points within the video. Thereby, we took into consideration that the processing of a target sign already begins during the preceding transition phase between the previous sign and the target sign (see Hosemann et al, 2013, Chapter 1). For each video, two linguists with a high 
expertise in DGS identified each trigger point independently. Conflicting classifications never differed by more than a single frame $(40 \mathrm{~ms})$ and were resolved by discussion. The critical moments for trigger setting were:

(a) Handshape change (hsc). This trigger was placed at the frame during the handshape change in which the hand was tensionless and neither the handshape of the previous sign nor the handshape of the target sign were identifiable.

(b) Target handshape (ths). This trigger point was defined as the first frame in which the target handshape was completely accessible, regardless of target orientation or location.

(c) Nonmanual cues ( $\mathrm{nmc}$ ). Verb agreement was additionally marked by the nonmanual components of eye gaze and head tilt towards the final $\mathrm{R}$-locus of the verb. This trigger point was defined as the first frame in which eye gaze towards the according R-locus was clearly identifiable and/or in which the head left its neutral position. If eye gaze and head tilt did not change in the same frame, the first frame with a nonmanual deviation (either eye gaze change or head tilt change) was picked. On average, the nmc-trigger occurred at almost the same time as the thstrigger. The mean time difference between both triggers lay only at $44 \mathrm{~ms}$ (i.e., one video frame), but with a standard deviation of $112 \mathrm{~ms}$ (range: $-320 \mathrm{~ms}-360 \mathrm{~ms}$ ). Thus, we decided to analyze the EEG signal to both trigger definitions independently. The nonmanual cue trigger occurred on average $202 \mathrm{~ms}$ (sd $128 \mathrm{~ms}$ ) prior to the sign onset trigger.

(d) Sign onset (on). Sign onset was defined as the first frame of the initial hold for the critical verb, when the target hand configuration reached the target location at body contact with the signer (in case of body anchored agreement verbs) or at the locus just in front of the signer (in case of non-body anchored agreement verbs).

The average durations from sign onset to sign offset were $429 \mathrm{~ms}$ (sd 168) for matching agreement verbs and $461 \mathrm{~ms}$ (sd 174) for mismatching agreement verbs.

For the statistical analysis of the ERP data, repeated measures analysis of variance (ANOVAs) were calculated with the factor CONDITION (match vs. mismatch) and topographical region of interest (ROI). Analyses were conducted using R (Team, 2012) and the ez package (Lawrence, 2012). Lateral 
ROIs were defined as follows: left-anterior (F3, F7, FC1, FC5, C3), rightanterior (F4, F8, FC2, FC6, C4), left-posterior (CP1, CP5, P3, P7, 01), and rightposterior (CP2, CP6, P4, P8, 02). For the analysis of the midline electrodes, FZ, CZ, CPZ, PZ, and OZ were each treated as individual ROIs. The statistical analysis was carried out in a hierarchical manner, i.e. only significant interactions $(p<0.05)$ were resolved. Probability values were Huynh-Feldt corrected when appropriate (Huynh \& Feldt, 1970).

\subsubsection{Exp. A: Results}

\section{ERP data 22}

Grand averages for sentences with agreement verb violation and for their matching control sentences are displayed in Figure 3.6 (correlated to trigger nonmanual cues) and in Figure 3.7 (correlated to trigger sign onset).

According to visual inspection there are two ERP effects: A right sided posterior effect, apparent at Figure 3.6, and a left sided anterior effect, apparent at Figure 3.7. The right posterior effect is most pronounced at trigger nonmanual cues and appears at the right posterior electrodes P8, P4, CP6, and 02 .

22 Unfortunately, the results of the behavioral data have not been evaluated up to the current moment. The following presentation of the results and their discussion is therefore based on the ERP data only. 


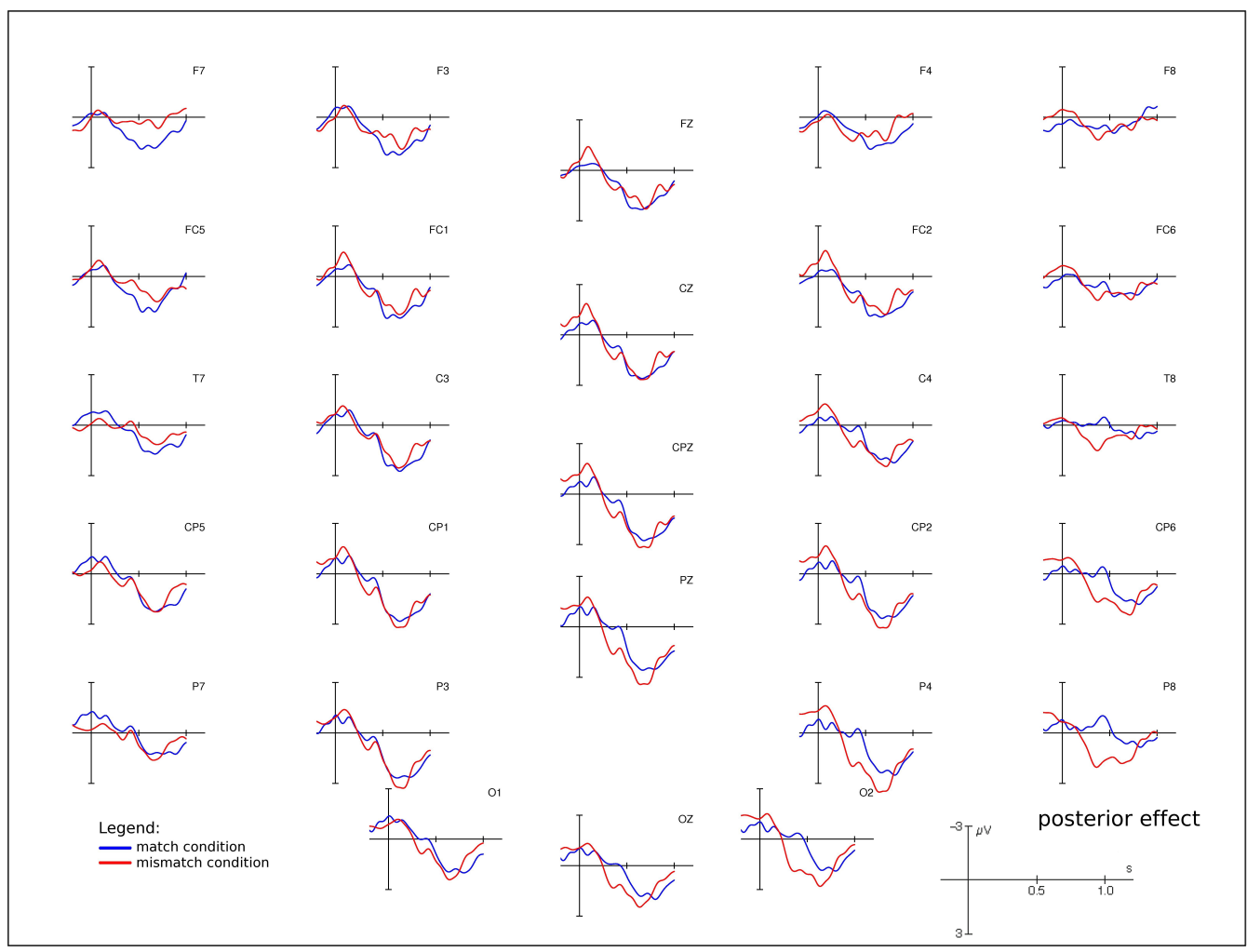

Figure 3.6 Grand average ERPs for matching (blue line) and mismatching (red line) agreement verbs, time-locked to the nonmanual cue trigger. Negativity plotted upwards.

Statistical analyses were conducted for the time window 220-570 ms and resulted in a CONDITION to ROI interaction: $F(1,17)=18.28, p=0.0000006$, and in a condition effect for the right-posterior ROI: $F(1,17)=15.99$, $p=0.00092$ for lateral electrodes. For midline electrodes the CONDITION to ROI interaction $(F(1,17)=5.94, p=0.00061)$ lead to significant effects at electrode Pz: $F(1,17)=6.41, p=0.021$, and electrode Oz: $F(1,17)=16.84$, $p=0.00074$.

The left anterior effect appears at left lateralized anterior electrodes F7, F3, FC5, FC1, and the central electrode $\mathrm{C} 3$ and displays a more negative-going wave for mismatching verbs (ending in $3 b$ instead of $3 a$ ) compared to their matching controls (ending in $3 \mathrm{a}$, the locus of the referent). The statistical analysis was conducted for the time window 300-600 ms relative to trigger 
sign onset and resulted in a CONDITION to ROI interaction: $F(1,17)=13.16$, $p=0.00015$, and in a condition effect for the left-anterior ROI: $F(1,17)=16.38, p=0.00084$ for lateral electrodes. Midline electrodes did not become significant after Huynh-Feldt correction (ps > 0.08).

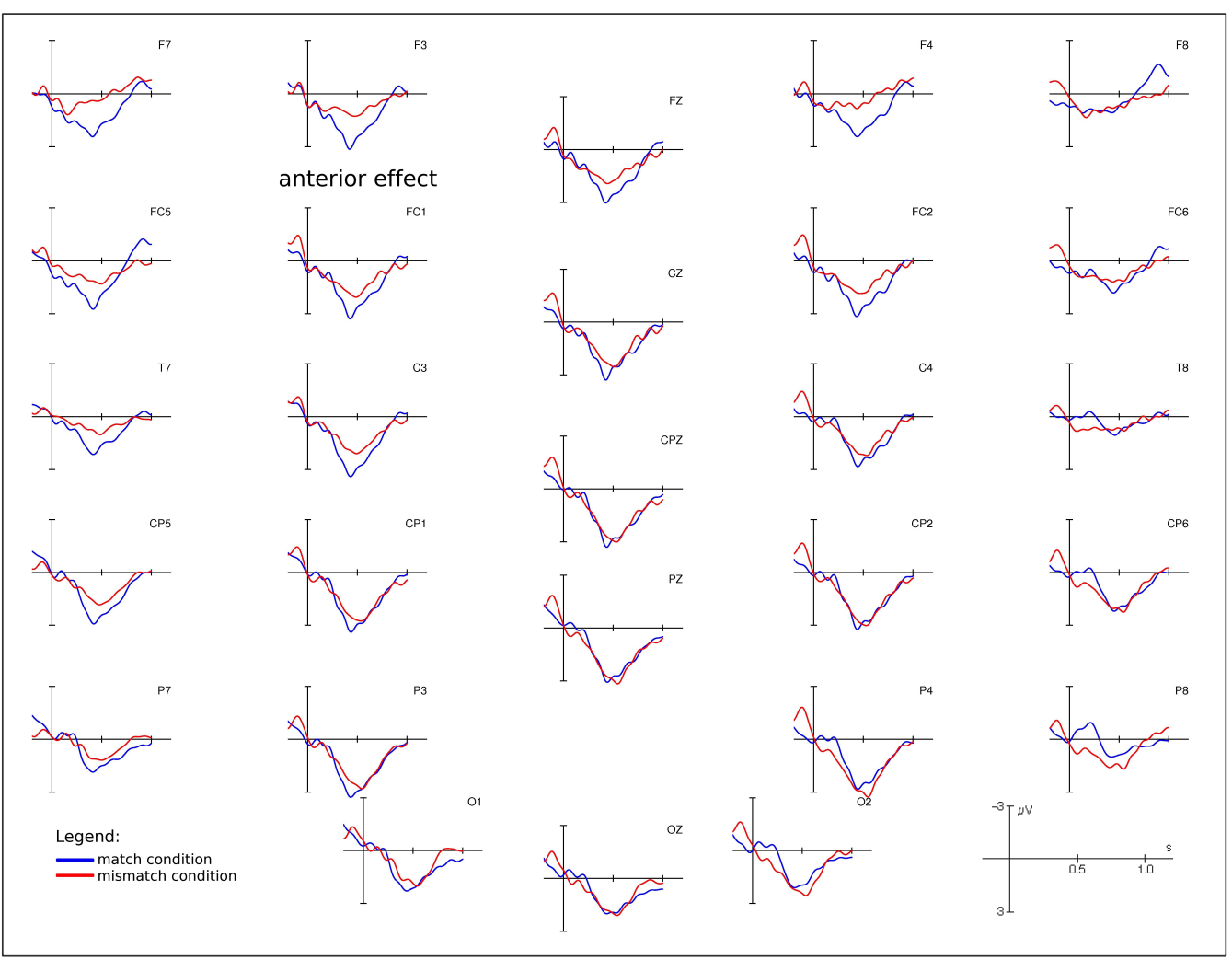

Figure 3.7 Grand average ERPs for matching (blue line) and mismatching (red line) agreement verbs, time-locked to the sign onset trigger. Negativity plotted upwards.

To sum up, agreement verbs ending in an unspecified R-locus ( $3 \mathrm{~b}$ ) in contrast to agreement verbs ending in an R-locus associated with a previously assigned referent (3a) elicited two ERP effects: a right posterior effect and a left anterior effect. The polarity and the functional interpretation of both effects remain uncertain at first sight. In the subsequent section we will discuss both effects, before comparing these results with those from Experiment $B$ on agreement violation with plain verbs in the general discussion in Section 3.4. 


\subsubsection{Exp. A: Discussion}

In contrast to Capek et al. (2009), who report an anterior negativity distributed most prominently over the right hemisphere followed by a broadly distributed P600 over posterior sites for unspecified verb agreement violation, we found two ERP effects that seem to be unrelated to one another. The right-posterior effect is most pronounced at trigger 'nonmanual cues' and appears in a time window of $220-570 \mathrm{~ms}$, while the left-anterior effect appears at trigger 'sign onset' in the time window 300-600 ms. Since trigger sign onset was time-locked on average $202 \mathrm{~ms}$ after the nonmanual cues trigger, it appears that the posterior effect at right lateral sites temporally evolved approximately 200-300 ms before the anterior effect evolved at left lateral sites. This indicates that both effects are not causally related and should thus be interpreted separately.

\subsubsection{The right-posterior effect}

The polarity of the effect cannot be clearly determined according to the results so far. Although the ERP waveform for the correct control condition (blue line) displays a negative peak at approximately $450 \mathrm{~ms}$, we assume this effect to be a more positive-going waveform for the mismatch condition (red line) relative to the control condition. The functional interpretation of this positivity effect is based on speculative grounds, since the number of ERP studies with unspecified verb agreement violation in sign languages is limited. We therefore provide possible explanations for this effect.

\section{$A$ violation of well-formedness}

Following the extended Argument Dependency Model (eADM) by Bornkessel \& Schlesewsky (2006), a first explanation of this posterior positivity effect is that it is elicited by a violation of well-formedness. According to this model of comprehending core constituents (i.e. verbs and their required arguments), the incremental comprehension processes follow three phases: In phase one, the current processing item is identified as verb or noun phrase argument. In 
phase two, the prominence of an NP is computed (as actor or undergoer) according to morphological information on case, position, animacy etc. Further, agreement information is assigned. If the computed item is a verb, its logical structure and agreement information are established and it is linked to arguments that have already been established. In the third phase, core relations and noncore relations are mapped and the NP/verb-structure is evaluated in terms of well-formedness or for possible repair processes (under the consideration of world knowledge, plausibility, and prosodic information of pitch accents, stress patterns, etc.) (c.f., Bornkessel \& Schlesewsky, 2006: p. 789-790). The notion of well-formedness is a mechanism that gradually appraises the acceptability of an item or structure in relation to its sentential and contextual environment.

In our experimental manipulation, we presented sentences like "MY FATHER IX $_{3 a}$ SOCCER FAN. NEXT MATCH DATE ${ }_{1}$ INFORM3b" in which the final agreement verb does not end at the R-locus associated with the object, but at an unspecified R-locus. A well-formed expression of verb agreement would be a verb ending on the same R-locus as the one associated with the object. But as Quer (2011) points out, the one referent to one R-locus ascription is not as straightforward in everyday sign language use as it is in theoretical terms. Quer (2011) refers to observations in Catalan Sign Language, in which, within a connected discourse, one referent can be referred to by the use of several Rloci. Quer (2011: p. 191) states that: "Directing the object pronoun towards a different location ( $3 \mathrm{~b}$, for instance) when we intend to refer to the discourse referent associated with locus $3 \mathrm{a}$ in that context is not ungrammatical, but just infelicitous." Therefore, an agreement verb not ending at the R-locus associated with the referent but at a different R-locus would not be interpreted as a grammatical mistake but rather as a violation of well-formedness.

This functional interpretation could be confirmed by a follow-up experiment. $\mathrm{R}$-locus $3 \mathrm{~b}$ is at the contralateral side of the signer opposite to R-locus $3 \mathrm{a}$ on the ipsilateral side of the signer. These locations in signing space typically mark an opposition or contrast of two referents (cf. Steinbach \& Onea, 2015). 
In the follow-up experiment, we would direct the agreement verb in the mismatch condition towards a neutral locus in signing space in front of the signer $\left(\ldots 1 \mathrm{INFORM}_{\mathbf{N}}\right)$ instead of towards R-locus $3 \mathrm{~b}$. If a 'wrong' final location of an agreement verb were a matter of well-formedness (instead of morphosyntactic violation), we would predict that this kind of violation elicits the same neurophysiologic response as we have observed here.

\section{Extra costs for context updating}

A second explanation of the posterior positivity effect is in terms of discourse updating as reported by Baumann \& Schumacher (2012) and Hung \& Schumacher (2012, 2014). Baumann \& Schumacher (2012) presented German monolinguals auditory sentences that varied with respect to information status of an entity (given versus new) and prosodic realization (accented versus deaccented). In the critical sentence, the information status of the $3^{\text {rd }}$ person target NP ("the winegrower") was varied by the previously set context sentence. The target NP was given, when it has already been introduced in the preceding context sentence. In contrast, the target NP was new, when it was not mentioned in the preceding context sentence. (11) shows an example from Baumann \& Schumacher (2012: p. 366-367).

(11) a. Frauke said that the lumberjack was not very cheerful.

She mentioned that the winegrower was very cheerful. [new]

b. Vivian talked about a winegrower in Baden.

She mentioned that the winegrower was very cheerful. [given]

ERPs time-locked to the noun elicited a biphasic N400 - Late Positivity pattern for new information compared to given information. They argue that the N400 reflects enhanced costs for linking new information to the previous discourse, and that the Late Positivity is caused by the listener's effort to update their discourse model. These effects were independent of an 
appropriate or inappropriate prosody and occurred for accented as well as deaccented nouns. Hung \& Schumacher (2012 and 2014) also found a biphasic N400 - Late Positivity pattern for a topic shift in sentence-medial and sentence-final position in Mandarin Chinese. Thus, a new topic in sentence-final position in the answer of a question-answer pair elicited a Late Positivity for context updating processes.

If we interpret our results in the light of this explanation, the posterior positivity effect was caused by additional processing costs for context updating. Agreement verbs do not only anaphorically agree with the location of a previously introduced referent, they can also mark the location of a new referent that is cataphorically introduced later in the discourse. Thus, in the mismatch condition, a referent $\mathrm{X}$ was introduced and associated with a locus in sentence one. However, in sentence two, the agreement verb ended at an unspecified locus and thereby introduced a new referent $Y$ that would have had to be explicated in a further context. Sentence two thus introduced a topic shift from referent $\mathrm{X}$ to a potential new referent $\mathrm{Y}$. The posterior positivity thus reflects enhanced processing costs for updating the situation model.

\section{A violation of presupposition}

A further explanation of the posterior positivity assumes that the kind of agreement violation is in fact a case of presupposition violation. The agreement verb ending at locus $3 \mathrm{~b}$ presupposes a referent that the verb can be linked with. The failure of this linking process in the mismatch condition caused the enhanced processing costs. ERP studies on spoken language pronoun resolution typically show a widely distributed negative deflection for ambiguous referents (Van Berkum et al., 1999a,b; see Van Berkum et al., 2007, and Nieuwland \& Van Berkum, 2008, for references).

They tested sentences with unambiguous and ambiguous pronouns as those in (12). 
(12) a. David shot at Linda as he jumped over the fence.

[unambiguous: 1 referent]

b. David shot at John as he jumped over the fence.

[ambiguous: 2 referents]

c. Anna shot at Linda as he jumped over the fence.

[failing: 0 referents]

For referentially ambiguous nouns or pronouns as in sentences like (b) compared to (a), Van Berkum et al. (1999a,b) and Van Berkum et al. (2004) found a widely distributed negative deflection, emerging at about $300 \mathrm{~ms}$ after noun/pronoun onset. They dubbed this referentially induced negativity the "Nref effect". However, for sentences like (c) with no available referent, Van Berkum et al. (2004) found no Nref effect but rather a P600. They explain that when people only found referents of a different gender than the pronoun available, they take this to be a syntactic problem.

Although the agreement violation in our experiment is not a violation of gender, it can be compared to Van Berkum et al.'s (2004) findings. Pronouns and verb agreement in sign languages are two related phenomena (Pfau \& Steinbach, 2006; Steinbach, 2011) that are both expressed by location overlap with a corresponding referent. A verb ending at an unspecified R-locus (like $3 \mathrm{~b}$ in our mismatch condition) presupposes a referent that has not been introduced in the discourse model. Thus, the reference of the verb ending is "empty", because there is no adequate referent available (similar to sentence 12c). This violation led to the posterior positivity effect in our results.

Van Berkum et al. (2003) showed for pronouns during sentence processing that we check our situation model already at a very early stage whether we have an adequate referent or not (see also Van Berkum et al., 2007). This can explain the early latency of the posterior positivity effect at 220-570 ms relative to the trigger nonmanual cues prior to the sign onset. As argued in Chapter 1 , the verification of top down expectations and bottom up information already takes place during the relatively long transition phases prior 
to the onset of a lexical sign. Thus, in the current study, the expectation on the agreement verb was a direction towards R-locus 3a in accordance with the location of the object. The very early nonmanual cues of eye gaze and head tilt either confirmed this expectation (in the match condition) or violated it, when gaze and head tilt were directed towards the R-locus $3 \mathrm{~b}$. This indicated a referent that did not fit in the situation model.

However, the posterior positivity was not broadly distributed as it is characteristic for Late Positivity effects. This could be the result of an interference with the left anterior effect evolving approximately 200-300 ms later.

\subsubsection{The left anterior effect}

As it is apparent in Figure 3.7, the left anterior effect appears most prominent at electrode F3 relative to the trigger sign onset. The polarity of the effect is not univocal in the sense whether the mismatch condition (red line) is relatively distinct compared to the match condition (blue line) or vice versa. We will further discuss both interpretations.

\section{A left anterior negativity effect}

If the left anterior effect reflects a violation of expectation on the direction of the agreement verb, mismatching verbs directed towards an unspecified Rlocus elicited a more negative deflection compared to matching verbs directing at the object location. Van Berkum et al (2003) report an N400 effect for adjectives that were incongruent with a formally set discourse. In comparison to the adjective "fast", the critical word "slow" elicited an N400 effect in the spoken sentence "Jane told her brother that he was exceptionally..." when the previous context made clear that the brother acted in fact very fast. They thus conclude that listeners match unfolding words very early with the wider discourse (see also Van Berkum et al., 2005, for unexpected nouns within a discourse). Similarly, the left anterior negativity in 
our results could reflect an expectation mismatch of the verb with the wider discourse (also Molinaro et al., 2011).

Whether or not the left anterior effect is a negativity in the sense of Van Berkum et al.'s (1999a) Nref effect is an open question. The Nreff effect, a widely distributed negative deflection around $300 \mathrm{~ms}$ post word onset, was not only found for ambiguous nouns, followed by a disambiguating relative clause (Van Berkum et al., 1999a,b), but also for ambiguous pronouns (Van Berkum et al., 2004) as described in Section 3.2.3.1. Nieuwland \& Van Berkum (2006) report an $\mathrm{N} 400$ for ambiguous pronouns relative to non-ambiguous pronouns, which was modulated by the contextual bias towards one of the two referents: The more bias towards one referent, the lesser the ambiguity between the two referents, and hence, the smaller the N400 effect. In this line of argumentation, the left anterior negativity in our results would indicate an ambiguity of potential reference: either the introduced referent from sentence one that was associated with a different locus, or a new referent that has not yet been established at that moment of sentence processing. However, since we explicitly assigned R-locus $3 \mathrm{a}$ with the $3^{\text {rd }}$ person referent in sentence one and we assume that R-loci in signing space are referentially distinct, the left anterior effect is not likely to be an Nref effect for ambiguous pronouns in the sense of Van Berkum et al. (2004).

A further explanation of the frontal anterior effect understands the match condition (blue line) to be distinct from the mismatch condition (red line) and thus interprets the effect as a positivity for correct verb agreement.

\section{A left anterior positivity effect}

According to visual inspection of the left anterior effect throughout it's time course over the several time-locked trigger positions during the transition phase (i.e., handshape change, target handshape, and nomanual cues), the effect is not identifiable throughout these trigger positions. Instead, relative to trigger sign onset, the effect peaks at approximately $400-450 \mathrm{~ms}$. This indicates that the sign onset was the related event that caused a positive deflection for matching agreement verbs compared to mismatching agree- 
ment verbs. One possible explanation for the effect is that it was not caused by verb or agreement processing, but is rather task-related and thus falls into the P300 family. In a series of ERP studies with hearing participants, researchers investigated a positive deflection, typically peaking around $300 \mathrm{~ms}$ post stimulus onset, that is related to the given task, the probability of the target, and is not primarily caused by language processing costs (Sutton et al,. 1965; Squires et al., 1975; for an overview see Kok, 2001, Polich, 2007). Whether or not the P300 composes a family of effects or comprises a solitary effect is still under debate. However, in the literature two distinct effects have been classified: P3a and P3b (Squires et al., 1975; Polich, 2007). The P3a appears typically in oddball-paradigm experiments when the "taskirrelevant" stimuli are somehow distinct from the "task-relevant" stimuli, e.g., in intensity or frequency. The P3a has a peak latency between 220-280 ms post stimulus onset and can be clearly differentiate from the P3b by its topographic distribution. While the P3a has a fronto-central distribution, the P3b originates from temporal-parietal activity and has a rather posterior distribution.

The P3b is also a positive deflection with a peak amplitude at about $300 \mathrm{~ms}$ post stimulus onset. It was first elicited in an oddball paradigm by the less probable task-relevant stimuli (a rare " $\mathrm{X}$ " that had to be detected in a series of "O"s). Further investigation showed that it is not the individual probability of the stimulus that has an impact on the P3b amplitude, but rather the categorical probability. That is, the less probable the task-stimulus is in relation to the overall non-task stimulus, the larger the P3b amplitude. The $\mathrm{P} 3 \mathrm{~b}$ is also influenced by the stimulus sequence in the sense that the amplitude is larger for a target when preceded by several non-targets compared to a target preceded by only a few non-targets. This led to the assumption that it is either the unexpected event and the need to update the situation model that causes the P3b (Donchin, 1981; Donchin \& Coles, 1988), or it is the awaitedness of the target stimulus that causes the effect (Verleger, 1988, 1998). In an experiment where participants had to recall words from a list of several words, Karis et al. (1984) observed a larger P300 component 
for recalled items. Thus, they argue that fundamental memory processes affect the P300 amplitude (see also Donchin, 1981).

If we interpret the left anterior effect as a positivity for agreement verbs that match in their final location with the R-locus of the related object referent, we could assume the effect to be a task related effect from the P300 family. The first sentence introduced and located a referent within the signing space. This raises an expectation in the second sentence on the final verb to agree with the object and thus with the R-locus $3 \mathrm{a}$, respectively. In the correct condition this expectation is affirmed. The positivity effect for the correct condition could thus reflect a confirmation of topic continuity or fundamental memory processes associated with the referent assigned to the corresponding R-locus.

However, the positivity effect observed here, with a frontal distribution, does not fulfill the classic criteria of a P3b with a posterior distribution. Up to now, there are no reported ERP studies investigating the causes and the topographic distribution of a P300 during sign language processing. Hence, the interpretation of the crucial effect can only be speculative until further research is provided.

To sum up, the anterior effect appears in the time window 300-600 ms relative to trigger sign onset and seems to be more pronounced for the match condition compared to the mismatch condition. Under the assumption that this is a positivity effect for the match condition it seems to reflect a confirmation of the expected topic continuity of the referent. Although the effect is not typically distributed like a P300 component, the interpretation remains tentative since there is now experimental experience on P300 effects in sign language processing. Under the assumption that the effect is a negativity for the mismatch condition, it reflects an expectation violation of the topic.

In conclusion, unspecified agreement violation in continuous sentence processing, as we have investigated here, did not elicit a biphasic pattern of LAN and P600 as typically found for morpho-syntactic agreement violation in 
spoken languages (c.f., Molinaro et al., 2011) or as has been reported by Capek et al. (2009) and Hänel-Faulhaber et al. (2014) in sign languages. Instead, we found a posterior positivity effect for the mismatch condition related to nonmanuel cues prior to the actual sign onset. Subsequently, a left anterior effect emerged in relation to the sign onset that can either be a negativity for the mismatch condition (reflecting an expectation violation) or a positivity for the correct condition (reflecting affirmation of topic continuity). Therefore, from a neurolinguistic perspective, agreement with agreement verbs is not a pure morpho-syntactic phenomenon, but is also pragmatically motivated. In a further investigation, we aimed to examine a more far-reaching case of agreement violation. Hence, we conducted an experiment with agreeing plain verbs.

\subsection{Experiment B:}

\section{Agreement violation with plain verbs}

Plain verbs are lexically specified verbs that have a lexically specified initial hold and a lexically specified final hold. They cannot inflect for person or number. In Experiment B, we artificially manipulated plain verbs so they in fact do inflect for a $3^{\text {rd }}$ person object. By that, we intended to create a morphosyntactic violation of plain verbs.

\subsubsection{Exp. B: Materials and methods}

\subsubsection{Participants}

In this experiment, a total of 18 deaf native signers of DGS ( 5 female, 13 male) participated as paid volunteers. Their ages ranged from 16 to 52 years (mean age: 31). All signers had deaf parents or DGS input before the age of three and reported DGS to be their native language. All participants were right-handed, had normal or corrected-to-normal vision and reported no history of neurological disorders. Two participants had to be excluded from the final 
data analysis due to experimenter fault, a further two participants had to be excluded due to excessive eye movement artifacts.

\subsubsection{Materials}

Equivalent to the experiment on agreement violation with agreement verbs, the materials in this experiment on agreement violation with plain verbs comprised 40 sentences for each of the critical conditions (match versus mismatch), as illustrated in example (10) in Section 3.1.4. Critical sentences consisted of three signs: a $1^{\text {st }}$ person pronoun, a $3^{\text {rd }}$ person referent, and a frequent plain verb. Sentences were checked for grammatical and semantic correctness, frequency and possible dialectical variation by two deaf native informants. Plain verbs were body anchored (such as LIKE and KNOW) as well as non-body anchored (such as PLAY and BUY). Again, the 40 sentences per condition were constructed using 10 verbs with 4 repetitions, respectively, in different sentence contexts.

The corresponding sentences between conditions were identical, except for the critical plain verb in sentence final position. In the match condition, plain verbs were performed in their lexically specified manner, comprising a holdmovement-hold syllable structure (HMH). However, in the mismatch condition, plain verbs began in their lexically specified manner, but the path movement of the verb was manipulated so that it extended towards R-locus $3 \mathrm{a}$ on the right side of the signer. Thus, we did not violate the $\mathrm{HMH}$ syllable structure by adding an additional movement. Instead, we instructed our informants to stretch the lexically specified movement into a deviant path towards R-locus 3a. In light of the experimental manipulation, it is important to mention that these constructed plain verbs do not exist in this phonological form. They have been created according to the agreement rule deduced from agreement verbs: A location overlap between the final-hold of the verb and the R-locus associated with a referent marks agreement with the object of the sentence. 
Parallel to Experiment A, we instructed our informants to mark the manual "agreement" additionally with the nonmanual components eye gaze and head tilt. Thus, the informants gazed towards locus 3a with plain verbs in the mismatch condition. In the match condition, gaze was continuously directed towards the camera. In any other respect, nonmanual actions were kept to a minimum within the range of natural signing.

Note that the moment of mismatch in plain verbs with agreement marking appeared in the middle of the verb. The initial hold and the beginning of the path movement were identical for verbs of both conditions. The critical difference between matching and mismatching verbs occurred during the movement path by a change in hand orientation, path direction, and eye gaze towards locus 3a. Figure 3.8 shows the two different path movements of a lexically specified plain verb (a) and the manipulated plain verb that agrees with locus 3a. A complete list of stimulus sentences is provided in Appendix A.

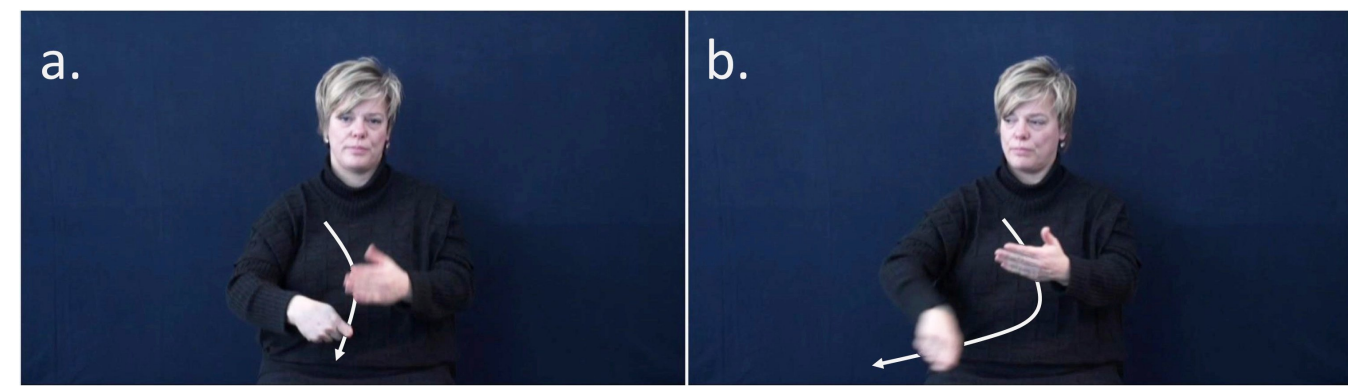

Figure 3.8 Video stills of the critical plain verb BUY, (a) with the lexical path movement, and (b) with the manipulated path movement ending at locus $3 a$.

With respect to construction, recording, and technical preparation, the production of the materials was identical to the production described in Experiment A in Section 3.2.1.2. In total, the videos had an average length of 5.26 seconds (sd 0.38). The 80 critical sentences resulting from this design were combined with 80 filler sentences, a separate experimental manipulation unrelated to the present design. A further 10 sentences were con- 
structed in the same way to function as practice sentences. They contained correct sentences with plain verbs and were not part of the actual experiment.

\subsubsection{Procedure}

The experimental procedure was identical to Experiment A, as described in Section 3.2.1.3.

\subsubsection{EEG recording}

The EEG recoding set up was identical to Experiment $A$, as described in Section 3.2.1.4.

\subsubsection{EEG data preprocessing and statistical analysis}

The EEG data preprocessing was identically set up as for Experiment A: The raw EEG was preprocessed using a $0.3-20 \mathrm{~Hz}$ band-pass filter, single subject ERP averages were calculated per condition and electrode from -200 to $1000 \mathrm{~ms}$ relative to four different trigger points (see below), and subsequently, grand averages were computed over all participants. Artifact rejection thresholds were at $40 \mu \mathrm{V}$.

Once again, we presented videos in an unsliced manner and analyzed ERPs according to four different time-locking trigger points: handshape change, sign onset, eye gaze onset, and mismatch onset. Trigger points were independently identified by two linguists with a high expertise in DGS.

(a) Handshape change (hsc). This trigger was placed at the frame during the handshape change in which the hand was tensionless and neither the handshape of the previous sign nor the handshape of the target sign were identifiable. 
(b) Sign onset (on). Sign onset was defined as the first frame of the initial hold for the critical verb, when the target hand configuration reached the target location.

(c) Eye gaze onset (ego). Since for the mismatch condition eye gaze towards location 3a was not always time aligned with the change in movement path, we decided to set two different trigger positions. This trigger was defined as the first frame in which eye gaze towards the according R-locus 3a was clearly identifiable. On average, this trigger occurred $260 \mathrm{~ms}$ after the sign onset.

(d) Mismatch onset (mmo). This trigger was placed at the first frame during the movement path of the verb in which the hand orientations left its lexically specified path and directed towards R-locus 3a. On average, this trigger occurred $460 \mathrm{~ms}$ after the sign onset and $200 \mathrm{~ms}$ after the eye gaze onset.

Note that triggers eye gaze onset and mismatch onset were defined according to criteria that apply only to mismatching verbs (correct plain verbs have no movement in gaze or a deviation in the movement path). In the matching verb counterparts, these triggers were time-locked to correlating moments during the path movement, where no deviation appeared. Average durations from sign onset to sign offset were $590 \mathrm{~ms}$ (sd 240) for matching plain verbs and $780 \mathrm{~ms}$ (sd 170) for mismatching plain verbs. This difference in sign length between conditions is based on the additional movement path for mismatching plain verbs.

For the statistical analysis of the ERP data, repeated measures analysis of variance (ANOVAs) were calculated with the factor CONDITION (match vs. mismatch) and topographical region of interest (ROI). Analyses were conducted using $\mathrm{R}$ (Team, 2012) and the ez package (Lawrence, 2012). Lateral ROIs were defined as follows: left-anterior (F3, F7, FC1, FC5, C3), right-anterior (F4, F8, FC2, FC6, C4), left-posterior (CP1, CP5, P3, P7, 01), and right-posterior (CP2, CP6, P4, P8, 02). For the analysis of the midline electrodes, FZ, CZ, CPZ, PZ, and OZ were each treated as individual ROIs. The statistical analysis was carried out in a hierarchical manner, i.e. only significant interactions $(p<0.05)$ were resolved. Probability values were Huynh-Feldt corrected when appropriate (Huynh \& Feldt, 1970). 


\subsubsection{Exp. B: Results}

\section{ERP data $^{23}$}

In Figure 3.9 to 3.11, we present grand averages for DGS sentences with agreement violation on plain verbs in comparison to their correct controls. In Figure 3.10, these are time-locked to the moment of mismatch during the path movement of the critical verb indicated by eye gaze towards the R-locus. In Figure 3.11, ERPs are time-locked to the moment of mismatch indicated by a deviation in the path movement. Further, Figure 3.9 displays grand averages for critical and control sentences, time-locked to the sign onset of the plain verb. Note that verbs were identical at the beginning in both conditions, thus identical frames could be triggered.

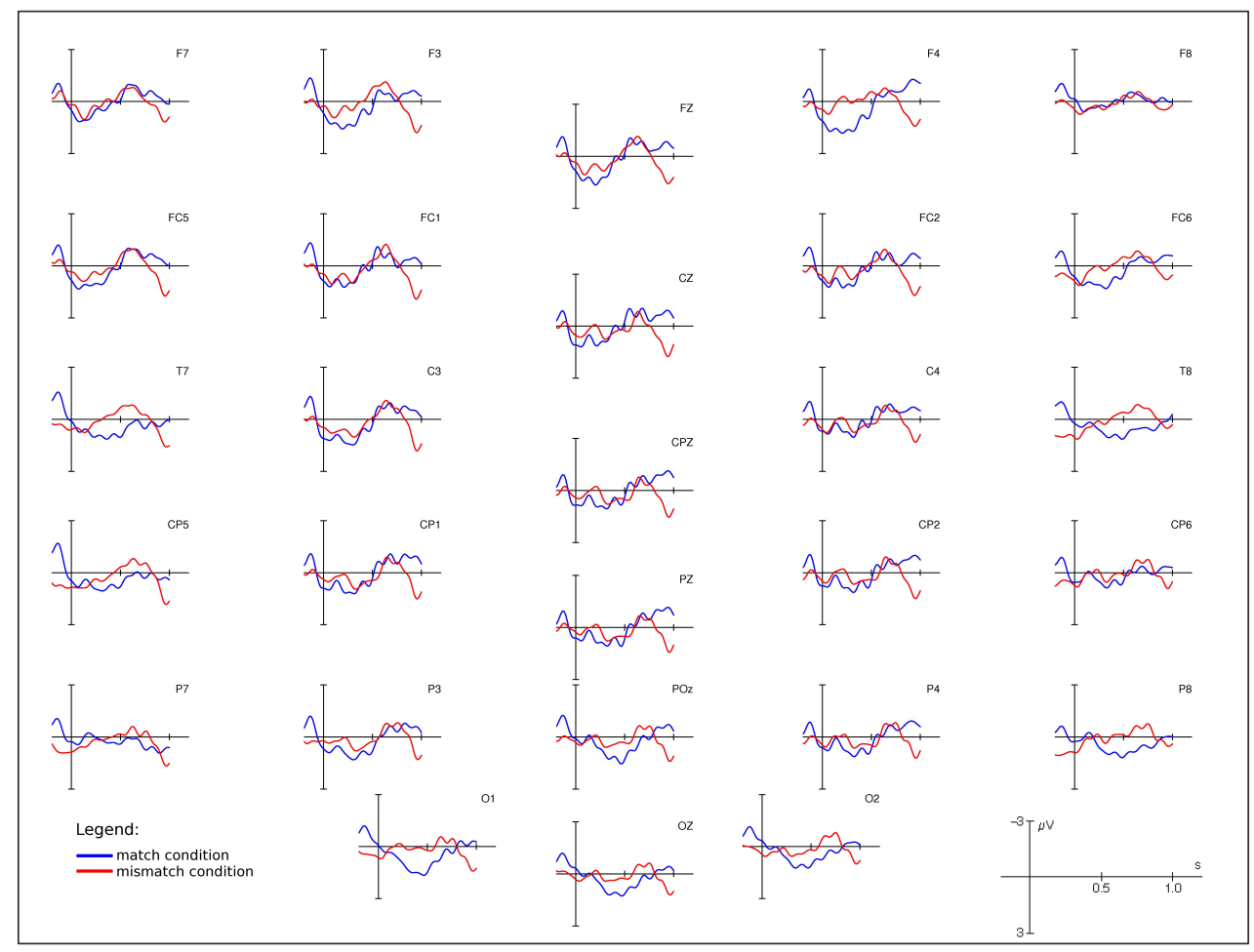

Figure 3.9 Grand average ERPs for matching (blue line) and mismatching (red line) plain verbs, time-locked to the trigger sign onset. Negativity plotted upwards.

23 The results of the behavioral data have not been evaluated up to the current moment. Thus, the following results and their discussion are based on the ERP data only. 
As is apparent from Figure 3.9, ERP waves relative to the sign onset do not differ in the time window of about 0-700 ms. Since the mismatch started approximately $260 \mathrm{~ms}$ (trigger eye gaze onset) and, respectively, $460 \mathrm{~ms}$ (trigger mismatch onset) into the sign, at both triggers appears a broadly distributed positive deflection for mismatching plain verbs compared to matching plain verbs. At trigger ego the positivity appears in the time window 470$820 \mathrm{~ms}$, while it appears in the time window 420-730 ms for trigger mmo.

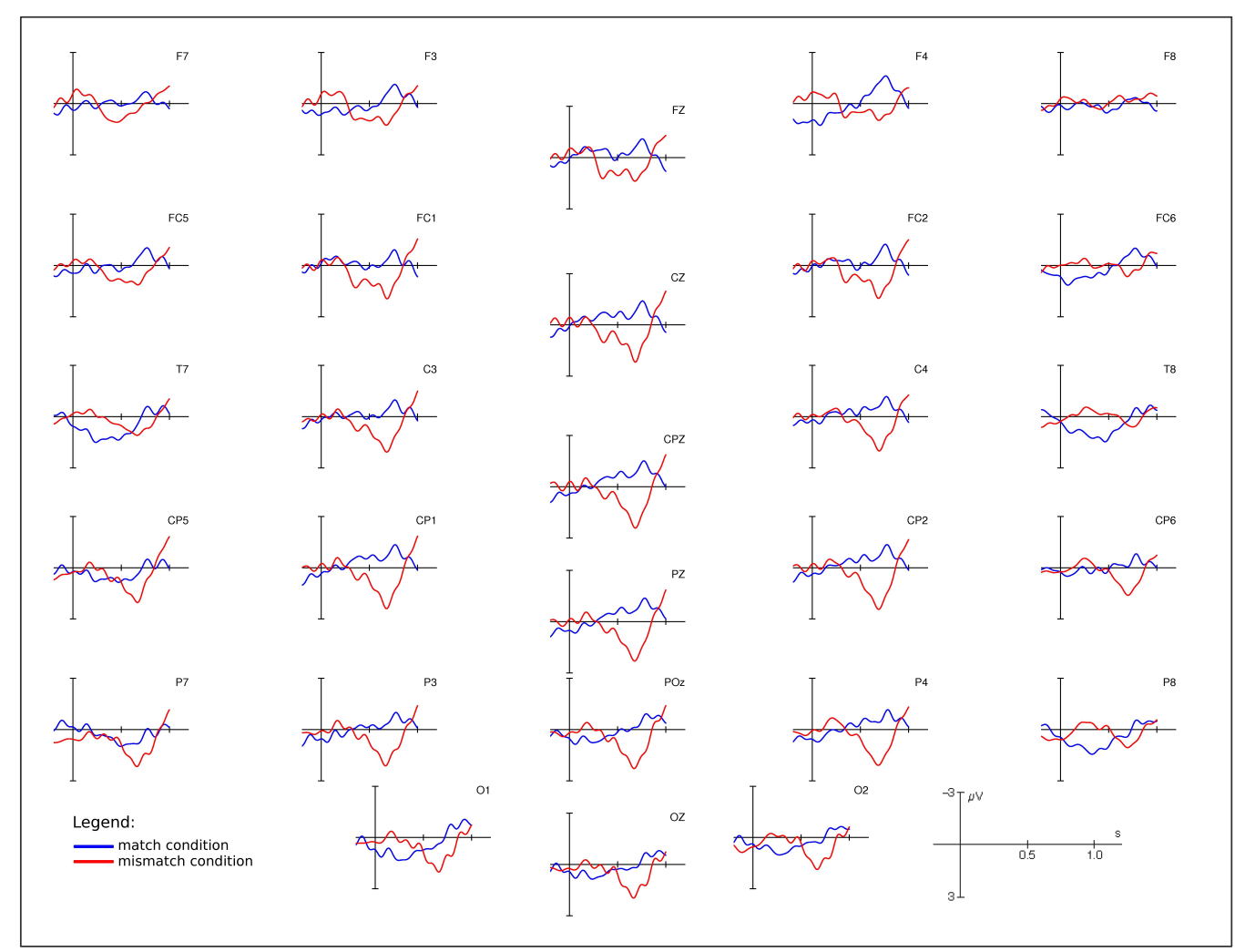

Figure 3.10 Grand average ERPs for matching (blue line) and mismatching (red linie) plain verbs, time-locked to the eye gaze onset trigger. Negativity plotted upwards.

This descriptive impression was confirmed by statistical analysis. For the 470-820 ms time window at trigger eye gaze onset, lateral electrodes show an over-all main effect for CONDITION: $F(1,13)=28.91, p=0.00012$; while midline electrodes show a CONDTION to ROI interaction $(F(1,13)=4.18$, 
$p=0.017)$, with the following significance for the electrodes Fz: $F(1,13)$ $=8.04, \quad p=0.014 ; \quad \mathrm{Cz}: F(1,13)=36.31, \quad p=0.000042 ; \quad \mathrm{CPz}: F(1,13)=53.96$, $p=0.0000056 ; \quad$ Pz: $F(1,13)=56.76, \quad p=0.0000042 ; \quad$ POz: $F(1,13)=12.69$, $p=0.0034 ;$ and Oz: $F(1,13)=8.56, p=0.011$.

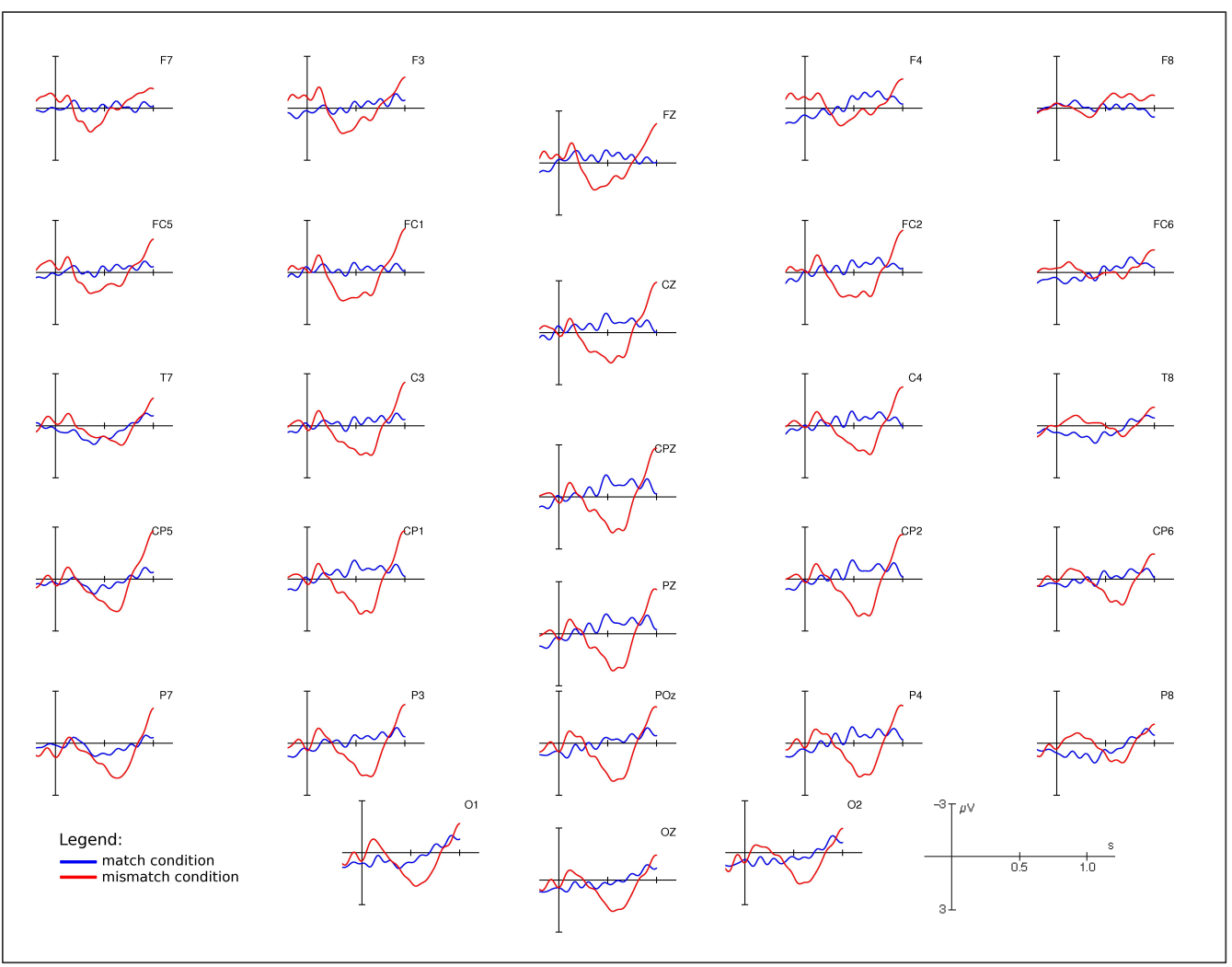

Figure 3.11 Grand average ERPs for matching (blue line) and mismatching (red linie) plain verbs, time-locked to the mismatch onset trigger. Negativity plotted upwards.

For the 420-730 ms time window at trigger mismatch onset, lateral electrodes also show an over-all main effect for CONDITION: $F(1,13)=24.64$, $p=0.00025$. Additionally, midline electrodes show a CONDTION to ROI interaction $(F(1,13)=4.79, p=0.017)$, with the following significance for the electrodes Fz: $F(1,13)=9.48, p=0.0087$; Cz: $F(1,13)=32.64, p=0.000071$; CPz: $F(1,13)=47.15, p=0.000011 ; \mathrm{Pz}: F(1,13)=42.63, p=0.000019 ;$ P0z: $F(1,13)=21.82, p=0.00043 ;$ and Oz: $F(1,13)=14.67, p=0.002$. 
According to visual inspection of trigger mismatch onset, there is an early negativity effect in the time window 40-190 ms post trigger onset, which did not become significant $(F(1,13)=3.24, p=0.095)$.

\subsubsection{Exp. B: Discussion}

In contrast to experiment $\mathrm{A}$ (agreement violation with agreement verbs), artificial agreement violation with plain verbs elicited a broadly distributed positivity effect in the time window of approximately $470-820 \mathrm{~ms}$ after the first nonmanual cue for the mismatch time-locked to trigger eyegaze onset. The effect also appeared relative to the manual mismatch in the time window 420-730 ms post trigger mismatch onset. This late positivity seems somewhat more pronounced at trigger ego, which is in some way confirmed by the statistical analysis: the main effect of condition is slightly more significant at trigger ego compared to trigger mmo (ego: $F(1,13)=28.91, p=0.00012$; mmo: $F(1,13)=24.64, p=0.00025)$. Whether or not the change in eye gaze or the change in path movement caused the ERP effect cannot clearly be recognized according to the present data.

Interestingly, artificially manipulated plain verbs did not elicit an N400 effect and thus were not interpreted as semantically incongruent. We therefore conclude that participants understood the semantics of the mismatch plain verbs. Nevertheless, this kind of violation did not elicit a classic biphasic pattern of LAN and P600, as no left anterior negativity was elicited. The broadly distributed positivity can indicate either a violation of well-formedness in the sense of the extended Argument Dependency Model (eADM) by Bornkessel \& Schlesewsky (2006) or it could reflect additional costs in the sense of context-updating as proposed by work from Schumacher (e.g., Baumann \& Schumacher, 2012).

In light of the eADM, the late positivity effect could be explained as caused by an evaluation process during the third phase of constituent comprehension. Note that the artificial manipulation of the verbs was not an additional 
movement after the lexically specified path movement of the verb (which would have led to a HMMH syllable structure). Rather, we instructed our informants to sign the verb with a lengthened path movement directing towards the locus 3a in signing space. Thus, we did not violate the $\mathrm{HMH}$ syllable structure of plain verbs. The lengthened path movement could thus be interpreted as an inaccurate or infelicitous production of the sign. Mismatching plain verbs would hence not be interpreted as grammatically incorrect but rather as less acceptable and not well-formed.

In the sense of Baumann \& Schumacher (2012) and Huang \& Schumacher (2012, 2014), the positivity effect could also reflect the need to update the situation model. Similarly to agreement verbs, which can assign R-loci to new referents prior to their explicit introduction, the ending of the plain verbs at locus 3a could indicate that the sentence is not completed and a further proposition is to follow. This would require enhanced processing costs for updating the context.

In an informal post-experimental behavioral feedback task, we asked nine deaf and two hard of hearing members of the Deaf community around Göttingen ( 5 female, 6 male) about their intuition on the sentences with incorrect plain verbs (e.g., I GRANDMA WAIT3a or I LAPTOP BUY3a). If their first intuitive feedback would have been in the sense of "what is coming next?" or "what is happening then?" this would have indicated that the sentences were not completed and that participants would expect a further proposition to fulfill the sentence. This kind of response would emphasize the explanation that context-updating caused the late positivity effect. In contrast, if the first feedback of signers would have been in the sense of "this is strange" or "this is wrong", it would support the idea that signers interpreted the sentences as felicitous and not well-formed. Further, if signers' first feedback would be in the sense of "I wait for grandma" or "I buy the laptop there", it would highly indicate that the extended path movement of the plain verbs towards locus $3 a$ in fact marks the object in a grammatical sense. In this case, the agreement principle we inferred from agreement verbs and transferred to plain verbs 
would in fact have been accepted as a (over generalized) rule. However, this kind of reaction requires a high competence in meta-linguistic awareness, and was not expected independent of the interpretation of artificial agreement in plain verbs. We showed five videos from the mismatch condition stimuli (i.e. with the artificially agreeing plain verbs) to the signers and asked the following question: "I will show you some videos with DGS sentences. Can you tell me what they mean?" The general first reaction was that these sentences are strange and do not exist in DGS. None of the signers asked how the sentences proceed. (See Appendix B for a written transcription of participants' reactions towards each video.) The behavioral feedback in this informal post-test suggests that the extended movement path in agreeing plain verbs is rather a violation of well-formedness than an indication for further context information.

Apart from this first study, agreement violation with plain verbs has not yet been investigated with online measuring methods. It is necessary to conduct further experiments on this topic, in order to shed light on the correlation between the underlying linguistic principle and the perhaps modality-specific ERP components. Thus, a definitive interpretation of the results is not possible at this moment. In the following section, we will address the modality-specific aspects of the present ERP findings and draw some conclusions on the linguistic status of agreement in sign languages.

\subsection{General discussion}

In Experiment $\mathrm{A}$ and Experiment $\mathrm{B}$, we investigated incorrect forms of agreement verbs and plain verbs in sentential contexts. In contrast to morpho-syntactic agreement violation studies in spoken languages (cf. Molinario et al., 2011) and in contrast to previous agreement violation studies in sign languages (Capek et al., 2009, Hänel-Faulhaber et al., 2014), incorrect agreement verbs and incorrect plain verbs in the present studies did not elicit a biphasic pattern of LAN and P600. Instead, unspecified agreement verbs 
elicited two rather independent effects, a right posterior and a left anterior effect, that seem to reflect separate cognitive processes. Artificially inflected plain verbs elicited a broadly distributed positive deflection. The possible functional interpretations of each effect are discussed in Sections 3.2.3 and 3.3.3, respectively. In this section, we will address the more general ideas of modality-specific properties of agreement in sign languages, which can be derived from the neurophysiologic results of the current and of the former ERP studies by Capek et al. (2009) and by Hänel-Faulhaber et al. (2014).

Based on the elicited ERP pattern in our results, the incorrect forms of agreement verbs and of plain verbs do not have to be a violation of agreement in a morpho-syntactical sense. As argued in Section 3.2.3, they could also describe a violation of information structure that is motivated, e.g., by an unexpected topic shift. So, in order to interpret the linguistic principle behind sign language agreement and the corresponding ERP pattern when this principle is violated, it is necessary to analyze the different kinds of sign language agreement violation separately and in more detail with respect to their semantic contribution.

One of the main differences between incorrect agreement verbs and incorrect plain verbs is that incorrect agreement verbs are violated by an inappropriate path movement and/or hand orientation. However, the incorrect forms can be appropriate in other contexts. Further, the path movement of a verb cannot be compared with a grammatical inflection suffix like -st in the German verb du gehst ('you are going'). Path movements and the initial- and final-hold of a verb transport semantic information that is evaluated within the sentential context. These aspects clearly distinguish the incorrect agreement verbs from the incorrect plain verbs. Since plain verbs and their path movement are lexically specified, the incorrect form - as constructed in the present experiment - is not appropriate in any context and is thus more difficult to reinterpret. To artificially transfer an agreement principle to a group of verbs that are basically not specified for agreement is a unique kind of agreement violation that cannot likewise be created with spoken language 
verbs. Thus, further investigations with plain verbs in incorrect forms are required.

In contrast, agreement verbs in incorrect forms can have an alternative interpretation. A sentence like "MY FATHER IX3a SOCCER FAN. NEXT MATCH DATE IINFORM3b", with an agreement verb ending in location $3 b$ instead of location $3 a$, is not understood in the sense that the semantic requirements of the sentence are fulfilled, and it just contains a grammatical mistake. Instead, the incorrect agreement verb provides semantic information that can be interpreted in the given sentential context. For example, that the signer will inform another person (that needs to be specified) about the date of the next match. The alternative English translation would thus be 'My father is a soccer fan. About the date of the next match, I will inform somebody else,...' As a consequence, different kinds of agreement violation imply different alternative interpretations that seem to evoke different ERP responses. Comparing the four recent studies on agreement violation in sign languages (i.e., Capek et al., 2009, Hänel-Faulhaber et al., 2014, and the two present studies), we can observe different forms of agreement violation. In Table 3.1, we list the forms of agreement violation with agreement verbs and with plain verbs that have been used in the present studies and in the discussed ERP studies. The setting gives information about the referents of the sentences (abbreviated with " $\mathrm{R}$ ") and the respective loci they were associated with. Further, we contrast the correct verb form with the incorrect verb form that was used instead, and list the corresponding ERP results.

Table 3.1 List of different kinds of agreement violation allocated to the studies they appeared in and the ERP effects they elicited ( $\mathrm{R}=$ referent; $\mathrm{L}=$ locus).

\begin{tabular}{|c|c|c|c|c|c|}
\hline Viol. & used in... & setting & corr. & incorr. & ERP effect \\
\hline (1) & $\begin{array}{l}\text { - Capek et al. } \\
\text { (unspecified agr.) } \\
\text { - Experiment A }\end{array}$ & $\begin{array}{l}\text { R1 = signer (L.1) } \\
\text { R2 = } 3^{\text {rd }} \text { person }(L .3 a)\end{array}$ & 1VERB3a & 1VERB3b & $\begin{array}{l}\text { - Capek: early anterior nega- } \\
\text { tivity ( } 200-360 \mathrm{~ms} \text {, right hemi- } \\
\text { spheric), late positivity (425- } \\
1200 \mathrm{~ms}) \text {; } \\
\text { - Experiment A: right posterior } \\
\text { positivity ( } 220-570 \mathrm{~ms}, \mathrm{nmc} \text { ), } \\
\text { left anterior effect ( } 300-600 \mathrm{~ms} \text {, } \\
\text { so), both seem not related. }\end{array}$ \\
\hline
\end{tabular}




\begin{tabular}{|c|c|c|c|c|c|}
\hline$(2)$ & $\begin{array}{l}- \text { Capek et al. } \\
\text { (reverse agr.) }\end{array}$ & $\begin{array}{l}\text { R1 = signer }(\mathrm{L} .1) \\
\mathrm{R} 2=3^{\text {rd }} \text { person }(\mathrm{L} .3 \mathrm{a})\end{array}$ & 1VERB3a & 3aVERB1 & $\begin{array}{l}\text { early anterior negativity (140- } \\
200 \mathrm{~ms} \text {, left hemispheric), } \\
\text { late positivity (475-1200 ms); }\end{array}$ \\
\hline (3) & $\begin{array}{l}- \text { Capek et al. } \\
\text { (reverse agr.) }\end{array}$ & $\begin{array}{l}\mathrm{R} 1=3^{\text {rd }} \text { person }(L .3 a) \\
R 2=3^{\text {rd }} \text { person }(L .3 b)\end{array}$ & 3aVERB $3 \mathrm{~b}$ & 3bVERB3a & $\begin{array}{l}\text { not analyzed separately } \\
\text { but together with (2) }\end{array}$ \\
\hline$(4)$ & $\begin{array}{l}- \text { Capek et al. } \\
\text { (unspecified agr.) }\end{array}$ & $\begin{array}{l}\mathrm{R} 1=3^{\text {rd }} \text { person }(\mathrm{L} .3 \mathrm{a}) \\
\mathrm{R} 2=3^{\text {rd }} \text { person }(\mathrm{L} .3 \mathrm{~b})\end{array}$ & 3aVERB3b & $\begin{array}{c}\text { 3bVERBN } \\
\text { (neut.) }\end{array}$ & $\begin{array}{l}\text { not analyzed separately } \\
\text { but together with (1) }\end{array}$ \\
\hline (5) & $\begin{array}{l}\cdot \text { Hänel- } \\
\text { Faulhaber } \\
\text { et al. }\end{array}$ & $\begin{array}{l}\mathrm{R} 1=3^{\text {rd }} \text { person }(L .3 \mathrm{a}) \\
\mathrm{R} 2=3^{\text {rd }} \text { person }(\mathrm{L} .3 \mathrm{~b})\end{array}$ & 3aVERB $3 \mathrm{~b}$ & NVERB1 & $\begin{array}{l}\text { left anterior negativity (400- } \\
600 \mathrm{~ms}) \\
\text { late posterior positivity }(1000- \\
1300 \mathrm{~ms})\end{array}$ \\
\hline (6) & - Experiment B & $\begin{array}{l}\mathrm{R} 1=3^{\text {rd }} \text { person } \\
\text { (default L.3a) }\end{array}$ & $\begin{array}{c}\text { VERB } \\
\text { (no agr.) }\end{array}$ & VERB3a & $\begin{array}{l}\text { broadly distributed positivity } \\
(420-730 \mathrm{~ms}, \text { ego })\end{array}$ \\
\hline
\end{tabular}

As can be seen from Table 3.1, double verb agreement - expressed by the path movement of the verb from subject location to object location - can be violated in structurally different ways: reverse path movement $(2,3)$, movement from correct subject location to an unspecified object location (1), movement from the object location towards an unspecified neutral location (4), or movement from an unspecified location towards the location of the signer who was not mentioned in the sentence (5). Each kind of agreement violation entails a different alternative interpretation. Consider an original example from Capek et al. 2009, taken from their Appendix. ${ }^{24}$

$$
\begin{aligned}
& \text { BOY PRO }_{3 a} \text {, GIRL PRO } 3 \mathrm{~b} \text { TWO-OF-THEM PLAY++ } \\
& \text { BOY___ GIRL PRO } 3 \text { b , WRONG PRO } 3 \text { bALL3b }
\end{aligned}
$$

'There was a boy and a girl and they were playing. The boy chased the girl, but oops, she fell.'

$$
\begin{array}{ll}
\text { Correct: } & { }_{3 a} \mathrm{CHASE}_{3 \mathrm{~b}} \\
\text { Reversed: } & { }_{3{ }_{3} \mathrm{CHASE}_{3 \mathrm{a}}} \\
\text { Unspecified: } & { }_{{ }_{3} \mathrm{CHASE}_{1}}
\end{array}
$$

\footnotetext{
${ }^{24}$ In the original example, locations were identified with letters "e" and "c". For reasons of
} uniformity, we replaced them with subscript " $3 \mathrm{a}$ " and " $3 \mathrm{~b}$ ". PRO is an index sign. 
The referent "boy" is associated with R-locus 3a on the right side of the signer, while the referent "girl" is associated with location $3 \mathrm{~b}$ on the left side of the signer. In the correct sentence, the verb CHASE moves from $3 a$ to $3 b$, meaning that the boy chases the girl. However, the sentence BOY ${ }_{3 b} \mathrm{CHASE}_{3 a}$ GIRL ... with a reverse verb movement could be interpreted as a passive construction, meaning 'the boy, the girl-chases-him'. Although, this would violate the subject-verb-object word order of ASL, it would be in line with the principle that verbs move from the location of the subject (actor) to the location of the object (undergoer). Similarly, the sentence BOY 3cCHASE3a GIRL ... with the verb beginning at a neutral locus, could be interpreted as 'the boy, somebody else chases him, the girl...'. Furthermore, in the incorrect sentence BOY ${ }_{3 a} \mathrm{CHASE}_{1}$ GIRL ... as Capek et al. (2009) used it for unspecified verb agreement, the verb ends at the location of the signer, which may lead to a relative clause interpretation: 'the boy, (who) he chases me, the girl...'. This shows that different forms of incorrect verb agreement can lead to different reinterpretations.

Accordingly, the ERP responses that were found in each study could be caused by different cognitive processes and should thus not be subsumed as a result of morphosyntactic agreement violation. That is to say, a late positivity effect for agreement violation in the one case could be originated from a different process than a late positivity effect found for a different kind of agreement violation. We therefore question, whether participants actually interpreted these agreement violations as syntactically anomalies, as proposed by Capek et al. (2009). „The distribution of the P600 effects for processing ASL syntactic violations is similar to that reported in studies of written and spoken language processing." (Capek et al., 2009: p. 8787). Within their design, Capek and colleagues combined different kinds of agreement violation under one category (e.g., unspecified agreement violation) and further used different types of verbs: typical agreement verbs like CHASE, verbs that do not take two animate arguments (and are thus not agreement verbs according to Rathman \& Marthurs', 2002 definition) like 
WASH in CAR... I MUST ${ }_{1} \mathrm{WASH}_{3}$ a, and backwards verbs like COPY. Thus, these different kinds of verbs and their different kinds of incorrect forms could have evoked different neurophysiological responses. Capek et al. (2009) themselves discuss that verbs in reverse agreement form can also be semantically incongruent: The sentence CAR CL3a... I MUST 3aWASH1 with reverse verb agreement can mean 'I must car-washes-me.' which describes also a semantic violation. However, they claim that participants interpreted this as a syntactic anomaly, because the neurophysiologic response to reverse agreement violation was a P600 instead of an N400 effect. This conclusion seems to be drawn too quickly, regarding that the one-to-one mapping of language related ERPs and linguistic domains is questionable. As reviewed and discussed by Bornkessel-Schlesewsky \& Schlesewsky (2008), an increasing number of studies report "semantic P600" effects. We therefore doubt that the late positivity effects found for sign language agreement violation (with agreement verbs) merely result from a syntactical violation.

In contrast, Hänel-Faulhaber et al. (2014) investigated a type of agreement violation in which agreement verbs moved from a neutral location opposite the signer (locus 3c) towards the location of the signer (locus 1). Thereby, the incorrect verb form marked an unspecified referent as the subject (at the neutral location) and the signer as the object. This verb form clearly conflicts with the two $3^{\text {rd }}$ person referents established in the sentence, because the initial and final hold of the verb both mark unassociated loci. Although the neutral location opposite the signer could be interpreted as marking the addressee (and thereby the participant of the experiment), the incorrect verb form, e.g. 3cNEEDLE1, can hardly be reinterpreted with the two previously introduced and locationally linked referents: BOY POINT $3 a$ GIRL POINT 3 b 3cNEEDLE 1 [...] (Hänel-Faulhaber et al., 2014: p. 7).

With regard to the ambiguity of R-loci, locations in signing space do not seem to be of the same ranking. Whereas the location at or close to the signer is always associated with the initiator of the utterance, in direct speech as well as in reported speech (cf. Lillo-Martin, 2012), the location opposite the signer 
is highly associated with the addressee. Locations marked for [-signer/-addressee] are further ranked within the context. For example, establishing one referent (or locative argument) on the right side of the signer (i.e. location 3a) and another referent (or locative argument) on the left side of the signer (i.e. location $3 \mathrm{~b}$ ), is often used to emphasize a contrast. On the contrary, the location in front of the signer is rather neutral when used for one referent or contrastive, when used in a set of three referents.

In follow-up ERP studies with agreement verbs, it would be interesting to contrast agreement verbs ending at locus 3a (in accordance with the established referent) with agreement verbs ending in neutral signing space (e.g., ... $\mathrm{IINFORM}_{\mathrm{N}}$ ). If locations are ranked with respect to the reference they can be associated with, agreement violation of this kind should evoke a different ERP response compared to the results from Experiment A. An agreement verb ending in neutral signing space might be associated with the established referent more easily than when it ends in an opposite location. Hence, we would expect to find a less pronounced ERP effect compared to correct agreement verbs. In a further step, one could investigate a clash between manual and nonmanual agreement. In a context where the $3^{\text {rd }}$ person referent is likewise associated with location $3 a$, the verb would end at location $3 \mathrm{~b}$ (i.e. manually incorrect agreement), but eye gaze, however, would be directed towards location $3 \mathrm{a}$, and would thereby display correct nonmanual agreement. This could emphasize a topic shift from the referent located at $3 \mathrm{a}$ to another (new) referent located at $3 \mathrm{~b}$. If so, we would expect to find a more prominent positivity effect due to context updating processes. 


\subsection{Summary and conclusions}

The status of agreement in sign languages is clearly distinct from that in spoken languages. That is to say, the agreement system in one spoken language compared to that of another spoken language share more core principles than the agreement systems of one spoken language and one sign language. In addition to the obvious modality-specific aspects of agreement in sign languages - the uniformity phenomenon and that only a subset of verbs agree -, neurophysiological responses to agreement violation in sign languages also emphasize the modality-specific status of the phenomenon. The two present ERP studies on agreement violation in German Sign Language show that agreement in sign language cannot be equated with morphosyntactic verb agreement in spoken languages. Agreement violation of agreement verbs can be realized in several different forms that imply different alternative interpretations. In contrast, agreement violation with plain verbs is a violation of the verbs lexical specification. Different kinds of agreement violation seem to evoke different ERP responses due to processing costs of either updating the situation context or evaluating the processed item as not well-formed. 


\section{Conclusion}

The time-course of processing different linguistic aspects in sign language sentences has been the linking subject of the ERP studies presented here. In contrast to previous ERP studies on sign language processing, the methodological conventions used in the present studies have been extended in order to present the stimulus sentences signed in one go and in a non-manipulated video. By using this kind of presentation, the processing of the sentences comes as close to processing sentences in a non-experimental environment. This methodological modification enabled us to get a different perspective on the time-course of sign language processing, which was emphasized by the distinctive characteristics of our findings.

In Chapter 1 we used a classic semantic expectation mismatch design with a sentential context raising an expectation on the final sign, which was either fulfilled or violated. Semantically unexpected signs engendered a biphasic N400 - late positivity pattern, whereas we argue that the late positivity effect is rather related to participants' responses. In contrast, the N400 was determined by the point at which a prediction error of the upcoming sign was recognized. Interestingly, the moment of error recognition was clearly prior to the sign onset and thus elicited by properties of the transition phase. By presenting sentences in non-manipulated videos, we could analyze the electrophysiological signal time-locked to different information sources during the transition phase: the handshape change, the moment when the target handshape is reached, and the sign onset. This led to our finding that the predictions on the upcoming sign, i.e. the processing of a sign, are already apparent during the transition phase towards that sign. If cutting out transition phases, the linguistic material would become either highly artificial or important information of the transition phases would be missing. We therefore claim that it is preferable to present signed stimulus material with transition phases rather than without. In the light of the importance of transition phases, some researchers have questioned the classic definition of 
"sign". However, we neither propose to re-define the theoretical definition of a sign, nor do we propose to assume transition phases to be part of the lexical entry of a sign. But in the context of neurolinguistic experiments, we claim that transition phases are highly relevant for the processing of signs.

In Chapter 2 we presented a study on cross-modal cross-language co-activation. We thereby addressed the question whether bimodal bilinguals coactivate representations of spoken/written words (L2) during the processing of sentences in their native sign language (L1). In a phonological priming design, we presented signed sentences with prime-target pairs that were either overt phonological minimal pairs in DGS, or covert orthographic minimal pairs of the corresponding German translations. Target signs that were related to their primes (across both conditions) engendered a reduced negative polarity in the electrophysiological signal compared to overall unrelated control signs. By presenting the prime-target pairs not only as isolated signs but rather within a sentential context, we could show that L2 language co-activation occurred across modalities even during processing the L1 sentence context. Thus, sign language representations and spoken language representations have to be strongly linked, despite the fact that they share no overt phonological form. This leads to the question as to what constitutes this link. In the discussion section of Chapter 2 , we consider a connection between sign representations and word representations via mouthing. The linguistic status of mouthings has not been established at this time and mouthings do not represent a basic phonological component of signs. However, we argue that a mouthing can be part of the sub-lexical information of a sign as well as it can be part of the sub-lexical information of a (spoken) word. Although the results of the study in Chapter 2 cannot clarify whether L2 language co-activation is mediated via a shared semantic node or via an activation of mouthing information, they point to the question whether an L1/L2 distinction can be drawn as clear-cut for deaf bimodal bilinguals. This question does not concern the language status of either the sign language or the spoken language. Of course, both languages can be acquired as a native or as a second language. The question rather addresses the underlying neural 
representation of languages. Can the distinction between a native sign language and a secondary acquired spoken/ written language be clear-cut, if representations of both languages are mediated via a shared visual representation of, for example, mouthing? How relevant are mouthings for the positive identification of a sign in German Sign Language? These questions remain to be addressed in future research. But the choice to investigate overt and covert phonological priming during whole sentence processing rather than in isolated signs enabled us to indicate these questions.

With respect to a neutral perspective on sign languages, in contrast to a spoken language biased perspective, Chapter 3 addressed a controversially discussed subject: the sign language agreement system. Agreement in sign languages is expressed via a location overlap of the beginning/ending of the verb with the location associated with its arguments (i.e. subject and/or object). In two ERP studies, we investigated the neurophysiologic responses to DGS sentences with either incorrect agreement verbs, or with incorrect, i.e. inflecting, plain verbs. In contrast to two previous studies on agreement violation in sign languages, which report comparable results to morpho-syntactic agreement violation in spoken languages, we did not find a typical biphasic pattern of LAN followed by a P600. Instead, agreement verbs ending at an unspecified location in signing space elicited two somewhat independent effects: a posterior positivity (220-570 ms relative to trigger nonmanual cues) and a left anterior effect (300-600 ms relative to trigger sign onset). Plain verbs presented contrary to their nature with $3^{\text {rd }}$ person agreement elicited a broadly distributed positive deflection, 420-730 ms post trigger mismatch onset. These results were discussed with regard to potential functional interpretations of extra costs for updating the situation context, or as a result of a violation in well-formedness. A structural analysis of the different types of agreement violation used in our studies as well as in the previous ERP studies by Capek et al. (2009) and Hänel-Faulhaber et al. (2014) revealed that the phenomenon called 'agreement' in sign languages is much more closely connected to pragmatic principles, e.g. topicality, than to concate- 
national agreement affixes in spoken languages. Although there are theoretical arguments as well as experimental indications that point to the similarities between the spoken and the sign language agreement systems, we emphasized in Chapter 3 the modality-specific and distinctive characteristics between both linguistic phenomena. By investigating agreement violation within a sentential context and analyzing different trigger positions in the continuous signing stream, we were able to show that previously drawn parallels on the morpho-syntactic status between sign and spoken language agreement might have been taken too quickly. From a neurolinguistic perspective, 'agreement' in sign languages should be investigated irrespective from a spoken language agreement bias.

The three studies presented in this thesis have one thing in common: By using a methodological modification of presenting stimulus sentences and analyzing the correlating ERPs, each study presents unrepeated results that originate in the modality specific characteristic of sign languages to be produced in a three-dimensional signing space with different articulators acting simultaneously: (1) unexpected movement trajectories during transition phases between signs elicited a recognition of prediction error; (2) mouthings seem to constitute a connective element between representations of signs and words; and (3) an unexpected final location of a verb engenders processing costs, perhaps for a reinterpretation of the discourse model. None of these attributes can be found in spoken language processing due to their way of articulation. The unique characteristic of sign languages to be articulated in a three-dimensional signing space must have an impact on the underlying cognitive processes in processing the language. Recent neurolinguistic research stresses the evolutionary origin of neural systems involved in language processing. Bornkessel-Schlesewsky et al. (2015) argue that the basic biological prerequisites of the neurobiology of human language are already established in nonhuman primates. Their model of a dual-stream architecture (ventral and dorsal stream) is based on the auditory cortical systems. Leonard et al. (2012) showed in an MEG study that processing signs in deaf individuals activated superior temporal regions surrounding auditory 
cortex, but that signs do not evoke activity directly in auditory cortex. The impact of the visual perception of sign languages (a) on the neurobiology of the language system and (b) on the time-course of processing sign languages has not yet been sufficiently investigated. Since ERP studies on sign languages are so rare, we do not know much about the modulations of N400 effects, about Late Positivity or P300 effects at the current moment.

Future research should follow two directions: First, ERP studies on sign language processing should be conducted with stimulus material in an nonmanipulated manner, including transition phases between signs. The natural appearance of the signed sentences is a prerequisite for an uninfluenced processing. Second, further fundamental research on the processing of lexical semantic aspects as well as sentence structural aspects of sign languages need to be conducted. For example, a concrete follow-up experiment on the results of Chapter 2 could be to investigate cross-modal language co-activation with a phono-semantic priming study. In order to examine the mediation of sign and word representations via a semantic node versus mouthing, one could present target signs (like CAT) that are preceded by a prime, which is phonologically related to a semantic associate of the target (prime: HOUSE, semantic associate of target: MOUSE). If spoken language co-activation during sign language processing is mediated through mouthing, the sign HOUSE should in fact prime the sign CAT. Instead, if spoken language co-activation is primarily mediated via a shared semantic node, HOUSE should not prime CAT, because CAT should not fall directly into the semantic associates of HOUSE. An ERP study on phono-semantic priming in sign language could shed light on the link between sign and spoken word representations in bimodal bilinguals. As it has been discussed within each chapter, this is the direction future ERP studies on the processing of sign languages could take.

The aim of this thesis was to present three individual ERP studies on phonological, morpho-syntactic, and semantic aspects during sentence processing in German Sign Language. An extended methodological approach 
revealed a distinctive perspective on the time-course and the modality specific aspects of processing a three-dimensional language. 


\section{References}

Altvater-Mackensen, N., \& Mani, N. (2011). Bilinguals activate words from both languages when listening to spoken sentences : Evidence from an ERP-study. In Proceedings of the 33rd Annual Meeting of the Cognitive Science Society.

Aronoff, M., Meir, I., \& Sandler, W. (2005). The paradox of sign language morphology. Language, 81, 301-344.

Barberà Altimira, G. (2012). The meaning of space in Catalan Sign Language (LSC). Reference, specificity and structure in signed discourse. Universitat Pompeu Fabra.

Barlow, M., \& Ferguson, C. A. (Eds.). (1988). Agreement in natural language. Approaches, theories, descriptions. Stanford, CA: CSLI Press.

Baumann, S., \& Schumacher, P. B. (2011). (De-)Accentuation and the Processing of Information Status: Evidence from Event-Related Brain Potentials. Language and Speech, 55(3), 361-381.

Battison, R. (1978). Lexical borrowing in American Sign Language. Silver Spring, MD: Linstok Press.

Benedicto, E., \& Brentari, D. (2004). Where did all the arguments go? Argument-changing properties of classifiers in ASL. Natural Language \& Linguistic Theory, 22, 743-810.

Bijeljac-Babic, R., Biardeau, A., \& Grainger, J. (1997). Masked orthographic priming in bilingual word recognition. Memory \& Cognition, 25(4), 447457.

Bishop, M., \& Hicks, S. (2005). Orange eyes: Bimodal bilingualism in hearing adults from deaf families. Sign Language Studies, 5(2), 188-230. doi:10.1353/sls.2005.0001

Blumenfeld, H. K., \& Marian, V. (2007). Constraints on parallel activation in bilingual spoken language processing: Examining proficiency and lexical status using eye-tracking. Language and Cognitive Processes, 22(5), 633660.

Bornkessel-Schlesewsky, I., Kretzschmar, F., Tune, S., Wang, L., Genç, S., Philipp, M., ... Schlesewsky, M. (2011). Think globally: Cross-linguistic variation in electrophysiological activity during sentence comprehension. Brain and Language, 117(3), 133-152.

Bornkessel-Schlesewsky, I., \& Schlesewsky, M. (2008). An alternative perspective on "semantic P600" effects in language comprehension. Brain Research Reviews, 59(1), 55-73. 
Bornkessel-Schlesewsky, I., Schlesewsky, M., Small, S. L., \& Rauschecker, J. P. (2015). Neurobiological roots of language in primate audition: common computational properties. Trends in Cognitive Sciences, 1-9.

Bornkessel-Schlesewsky, I., Staub, A., \& Schlesewsky, M. (n.d.). The timecourse of sentence processing in the brain. In G. S. Hickok \& S. L. Small (Eds.), Neurobiology of Language (pp. 1-40). Elsevier.

Bourguignon, N., Drury, J. E., Valois, D., \& Steinhauer, K. (2012). Decomposing animacy reversals between agents and experiencers: an ERP study. Brain and Language, 122, 179-189.

Braem, P. B., \& Sutton-Spence, R. (Eds.). (2001). The hands are the head of the mouth: The mouth as articulator in sign languages. Hamburg: Signum.

Brentari, D. (1990). Theoretical foundations of American Sign Language phonology. University of Chicago.

Brentari, D. (1998). A prosodic model of sign language phonology. Cambridge: MIT Press.

Brentari, D. (2012). Phonology. In R. Pfau, M. Steinbach, \& B. Woll (Eds.), Sign Language. An international handbook (HSK) (pp. 21-54). Berlin, Boston: De Gruyter Mouton.

Brouwer, H., Fitz, H., \& Hoeks, J. (2012). Getting real about semantic illusions: rethinking the functional role of the P600 in language comprehension. Brain Research, 1446, 127-143.

Burton, M. W. (1992). Syllable priming in auditory word re-cognition. In Poster session presented at the 33rd Annual Meeting of the Psychonomic Society, St. Louis, MO.

Camblin, C. C., Gordon, P. C., \& Swaab, T. Y. (2007). The interplay of discourse congruence and lexical association during sentence processing: evidence from ERPs and eye tracking. Journal of Memory and Language, $56,103-128$.

Canseco-Gonzales, E., Brehm, L., Brick, C. A., Brown-Schmidt, S., Fischer, K., \& Wagner, K. (2010). Carpet or Cárcel: The effect of age of acquisition and language mode on bilingual lexical access. Language and Cognitive Processes, 25(5), 669-705.

Capek, C., Campbell, R., \& Woll, B. (2008). The bimodal bilingual brain: fMRI investigations concerning the cortical distribution and differentiation of signed language and speechreading. Rivista Di Psicolinguistica Applicata, 3, 97-112.

Capek, C. M., MacSweeney, M., Woll, B., Waters, D., McGuire, P. K., David, A. S., ... Campbell, R. (2008). Cortical circuits for silent speechreading in deaf and hearing people. Neuropsychologia, 46(5), 1233-1241. 
Capek, C., Waters, D., Woll, B., MacSweeney, M., Brammer, M. J., McGuire, P. K., ... Campbell, R. (2008). Hand and mouth: Cortical correlates of lexical processing in British Sign Language and speechreading English. Journal of Cognitive Neuroscience, 20(7), 1220-1234.

Capek, C. M., Grossi, G., Newman, A. J., McBurney, S. L., Corina, D., Roeder, B., \& Neville, H. J. (2009). Brain systems mediating semantic and syntactic processing in deaf native signers: biological invariance and modality specificity. Proceedings of the National Academy of Sciences of the United States of America (PNAS), 106(21), 8784-8789.

Carreiras, M., Gutiérrez-Sigut, E., Baquero, S., \& Corina, D. (2008). Lexical processing in Spanish sign language (LSE). Journal of Memory and Language, 58(1), 100-122.

Casey, S., \& Emmorey, K. (2008). Co-speech gesture in bimodal bilinguals. Language and Cognitive Processes, 24(2), 290-312.

Clark, L., \& Grosjean, F. (1982). Sign recognition processes in American Sign Language: The effect of context. Language and Speech, 25, 325-339.

Connolly, J.F., \& Phillips, N.A. (1994). Event-related potential components reflect phonological and semantic processing of the terminal word of spoken sentences. Journal of Cognitive Neuroscience, 6, 256-266.

Corbett, G. (2006). Agreement. Cambridge, UK: Cambridge University Press.

Corina, D.P. (1992). Syllable priming and lexical representations: Evidence from experiments and simulations. In D. P. Corina (Ed.), Proceedings of the 16th Annual Conference of the Cognitive Science Society (pp. 779784). Hillsdale, NJ: Erlbaum.

Corina, D. (2000). Some observations regarding paraphasia in American Sign Language. In K. Emmorey \& H. Lane (Eds.), The signs of language revisited: An anthology to honor Ursula Bellugi and Edward Klima (pp. 493-507). Mahwah, NJ: Lawrence Erlbaum Associates.

Corina, D. P., \& Hildebrandt, U. C. (2002). Psycholinguistic investigations of phonological structure in ASL. In R. P. Meier, K. Cormier, \& D. QuintoPozos (Eds.), Modality and structure in signed and spoken languages (pp. 88-111). Cambridge: Cambridge University Press.

Corina, D., \& Spotswood, N. (2012). Neurolinguistics. In R. Pfau, M. Steinbach, \& B. Woll (Eds.), Sign Language. An international handbook (HSK) (pp. 739-761). Berlin, Boston: De Gruyter Mouton.

Coulsen, S., King, J.W., \& Kutas, M. (1998). ERPs and domain specificity: beating a straw horse. Language and Cognitive Processes, 13, 653-672.

De Groot, A.M.B., Delmaar, P., \& Lupker, S. J. (2000). The processing of interlexical homographs in translation recognition and lexical decision: 
Support for nonselective access to bilingual memory. Quarterly Journal of Experimental Psychology, 53, 397-428.

DeLong, K.A., Urbach, T.P., \& Kutas, M. (2005). Probabilistic word preactivation during language comprehension inferred from electrical brain activity. Nature Neuroscience, 8, 1117-1121.

Dijkstra, T., \& Heuven, W. J. B. van. (2002). The architecture of the bilingual word recognition system: From identification to decision. Bilingualism: Language and Cognition, 5(03), 175-197.

Dikker, S., \& Pylkkänen, L. (2011). Before the N400: effects of lexical-semantic violations in visual cortex. Brain and Language, 118, 23-28.

Dikker, S., Rabagliati, H., Farmer, T. A., \& Pylkkänen, L. (2010). Early occipital sensitivity to syntactic category is based on form typicality. Psychological Science, 21, 629-634.

Donati, C., \& Branchini, C. (2013). Challenging linearization: Simultaneous mixing in the production of bimodal bilinguals. In I. Roberts \& $\mathrm{T}$. Biberauer (Eds.), Challenges to linearization. Berlin, Boston: Mouton de Gruyter.

Donchin, E. (1981). Surprise!... Surprise? Psychophysiology, 18(5), 493-513.

Donchin, E., \& Coles, M. G. H. (1988). Is the P300 component a manifestation of context updating? Behavioral and Brain Sciences, 11, 357-374.

Dye, M. W. G. (2012). Processing. In R. Pfau, M. Steinbach, \& B. Woll (Eds.), Sign Language. An international handbook (HSK) (pp. 687-711). Berlin, Boston: De Gruyter Mouton.

Dye, M.W.G., \& Shih, S.-I. (2006). Phonological priming in British Sign Language. In L.M. Goldstein, D.H. Whalen, \& C.T. Best (Eds.), Laboratory Phonology 8: Varieties of Phonological Competence. (pp. 243-263). Berlin: Mouton de Gruyter.

Emmorey, K. (1989). Auditory morphological priming in the lexicon. Language and Cognitive Processes, 4, 73-92.

Emmorey, K. (2002). Language, cognition, and the brain: Insights from sign language research. Mahwah, NJ: Lawrence Erlbaum.

Emmorey, K. (2003). The neural systems underlying sign language. In M. Marschark \& P. E. Spencer (Eds.), The Oxford handbook of deaf studies, language, and education, Vol. 1 (pp. 361-375). Oxford: Oxford University Press.

Emmorey, K., Borinstein, Helsa, B., Thompson, R., \& Gollan, T. H. (2008). Bimodal bilingualism. Bilingualism: Language and Cognition, 11(1), 4361. 
Emmorey, K., \& Corina, D. (1990). Lexical recognition in sign language: Effects of phonetic structure and morphology. Perceptual and Motor Skills, 71, 1227-1252.

Emmorey, K., Damasio, H., McCullough, S., Grabowski, T., Ponto, L.L.B., Hichwa, R. D., \& Bellugi, U. (2002). Neural systems underlying spatial language in American Sign Language. NeuroImage, 17(2), 812-824.

Emmorey, K., Grabowski, T., McCullough, S., Damasio, H., Ponto, L.L.B., Hichwa, R.D., \& Bellugi, U. (2003). Neural systems underlying lexical retrieval for sign language. Neuropsychologia, 41(1), 85-95.

Federmeier, K.D. (2007). Thinking ahead: the role and roots of prediction in language comprehension. Psychophysiology, 44, 491-505.

Federmeier, K.D., Kutas, M., \& Schul, R. (2010). Age-related and individual differences in the use of prediction during language comprehension. Brain and Language, 115, 149-161.

Federmeier, K.D., \& Laszlo, S. (2009). Time for meaning: electrophysiology provides insights into the dynamics of representation and processing in semantic memory. In B. H. Ross (Ed.), Psychology of learning and motivation (Vol. 51, pp. 1-44). Burlington: Academic Press.

Frisch, S., \& beim Graben, P. (2007). The electrophysiology of vegetable language: A case study. Journal of Irreproducible Results, 50(3), 25-27.

Glück, S., \& Pfau, R. (1998). On classifying classification as a class of inflection in German Sign Language. In Proceedings of ConSole (pp. 1-16).

Greenhouse, S. W., \& Geisser, S. (1959). On methods in the analysis of profile data. Psychometrika, 24, 95-112.

Grosjean, F. (1981). Sign and word recognition: A first comparison. Sign Language Studies, 32, 195-220.

Grossi, G., Semenza, C., Corazza, S., \& Volterra, V. (1996). Hemispheric specialization for sign language. Neuropsychologia, 34(7), 737-740.

Grosvald, M., Gutierrez, E., Hafer, S., \& Corina, D. (2012). Dissociating linguistic and non-linguistic gesture processing: Electrophysiological evidence from American Sign Language. Brain and Language, 121(1), $12-24$

Grote, K., \& Linz, E. (2003). The influence of sign language iconicity on semantic conceptualization. In W. G. Müller \& O. Fischer (Eds.), From sign to signing: Iconicity in language and literature (Vol. 3). Amsterdam: John Benjamins.

Gunter, T.C., Friederici, A.D., \& Schriefers, H. (2000). Syntactic gender and semantic expectancy: ERPs reveal early autonomy and late interaction. Journal of Cognitive Neuroscience, 12, 556-568. 
Gutiérrez, E., Müller, O., Baus, C., \& Carreiras, M. (2012). Electrophysiological evidence for phonological priming in Spanish Sign Language lexical access. Neuropsychologia, 50(7), 1335-1346.

Gutiérrez, E., Williams, D., Grosvald, M., \& Corina, D. (2012). Lexical access in American Sign Language: An ERP investigation of effects of semantics and phonology. Brain Research, 1468, 63-83.

Hänel-Faulhaber, B., Skotara, N., Kügow, M., Salden, U., Bottari, D., \& Röder, B. (2014). ERP correlates of German Sign Language processing in deaf native signers. BMC Neuroscience, 15(62), 1-11. doi:10.1186/14712202-15-62.

Hanson, V.L. (1982). Short-term recall by deaf signers of American Sign Language: implications of encoding strategy for order recall. Journal of Experimental Psychology: Learning, Memory, and Cognition, 8(6), 572583.

Hanson, V.L., \& Feldman, L.B. (1989). Language specificity in lexical organization: evidence from deaf signers' lexical organization of American Sign Language and English. Memory \& Cognition, 17(3), 292301.

Henrich, J., Heine, S.J., \& Norenzayan, A. (2010a). Most people are not WIERD. Nature, 466, 29.

Henrich, J., Heine, S. J., \& Norenzayan, A. (2010b). The weirdest people in the world? The Behavioral and Brain Sciences, 33(2-3), 61-83; commentary 83-135.

Herrmann, A., \& Steinbach, M. (2007). Wenn ich nicht ich ist. Zitieren in Gebärdensprachen. In E. Brendel, J. Meibauer, \& M. Steinbach (Eds.), Zitat und Bedeutung. Linguistische Berichte Sonderheft (pp. 153-179). Hamburg: Buske.

Herrmann, A., \& Steinbach, M. (2012). Quotation in sign languages - A visible context shift. In I. van Alphen \& I. Buchstaller (Eds.), Quotatives: crosslinguistic and cross-disciplinary perspectives (pp. 203-228). Amsterdam: Benjamins.

Heuven, W.J.B. van, Dijkstra, T., \& Grainger, J. (1998). Orthographic neighborhood effects in bilingual word recognition. Journal of Memory and Language, 39, 458-483.

Hoeks, J.C.J., Stowe, L. A., \& Doedens, G. (2004). Seeing words in context: the interaction of lexical and sentence level information during reading. Cognitive Brain Research, 19, 59-73.

Hohenberger, A., Happ, D., \& Leuninger, H. (2002). Modality-dependent aspects of sign language production: Evidence from slips of the hands and their repairs in German Sign Language. In R. P. Meier, K. Cormier, \& 
D. Quinto-Pozos (Eds.), Modality and structure in signed and spoken languages (pp. 112-142). Cambridge: Cambridge University Press.

Hohenberger, A., \& Leuninger, H. (2012). Production. In R. Pfau, M. Steinbach, \& B. Woll (Eds.), Sign Language. An international handbook (HSK) (pp. 711-738). Berlin, Boston: De Gruyter Mouton.

Holcomb, P. J., \& Neville, H. J. (1991). Natural speech processing: An analysis using event-related brain potentials. Psychobiology, 19(4), 286-300.

Hosemann, J. (2011). Eye gaze and verb agreement in German Sign Language. A first glance. In A. Herrmann \& M. Steinbach (Eds.), Nonmanuals in sign languages. Special Issue of Sign Language \& Linguistics (Vol. 14, pp. 7693). John Benjamins.

Hosemann, J., Herrmann, A., Steinbach, M., Bornkessel-Schlesewsky, I., \& Schlesewsky, M. (2013). Lexical prediction via forward models: N400 evidence from German Sign Language. Neuropsychologia, 51(11), 22242237.

Hung, Y.-C., \& Schumacher, P. B. (2012). Topicality matters: Position-specific demands on Chinese discourse processing. Neuroscience Letters, 511(2), 59-64.

Hung, Y.-C., \& Schumacher, P. B. (2014). Animacy matters: ERP evidence for the multi-dimensionality of topic-worthiness in Chinese. Brain Research, 36-47.

Husain, F. T., Patkin, D. J., Thai-Van, H., Braun, A. R., \& Horwitz, B. (2009). Distinguishing the processing of gestures from signs in deaf individuals: An fMRI study. Brain Research, 140-150.

Huynh, H., \& Feldt, L. S. (1970). Conditions under which the mean-square ratios in repeated measurement designs have exact F-distributions. Journal of the American Statistical Association, 65, 1582-1589.

Jantunen, T. (2013). Signs and transitions: Do they differ phonetically and does it matter? Sign Language Studies, 13(2), 211-237.

Jantunen, T. (2015). How long is the sign? Linguistics, 53(1), 93-124.

Johnston, T., \& Schembri, A. (2007). Australien Sign Language: An introduction to sign language linguistics. Cambridge: Cambridge University Press.

Karis, D., Fabiani, M., \& Donchin, E. (1984). "P300" and memory: Individual differences in the von Restorff effect. Cognitive Psychology, 16, 117-216.

Kim, A., \& Osterhout, L. (2005). The independence of combinatory semantic processing: evidence from event-related potentials. Journal of Memory and Language, 52, 205-225.

Klann, J. (2012). Psycholinguistik und Neurolinguistik: Verarbeitung und Repräsentation von Gebärdensprache im Gehirn. In H. Eichmann, M. 
Hansen, \& J. Heßmann (Eds.), Handbuch Deutsche Gebärdensprache (pp. 271-292). Signum.

Klima, E. S., \& Bellugi, U. (1979). The signs of language. Cambridge, London: Harvard University Press.

Kok, A. (2001). On the utility of P3 amplitude as a measure of processing capacity. Psychophysiology, 38, 557-577.

Kolk, H. H. J., Chwilla, D. J., van Herten, M., \& Oor, P. J. (2003). Structure and limited capacity in verbal working memory: a study with event-related potentials. Brain and Language, 85, 1-36.

Kretzschmar, F. (2010). The electrophysiological reality of parafoveal processing: On the validity of language-related ERPs in natural reading. University of Marburg.

Kretzschmar, F., Bornkessel-Schlesewsky, I., \& Schlesewsky, M. (2009). Parafoveal vs. foveal N400s dissociate spreading activation from contextual fit. NeuroReport, 20, 1613-1618.

Kroll, J. F., \& de Groot, A. M. B. (Eds.). (2005). Handbook of bilingualism: Psycholinguistic approaches. New York: Oxford University Press.

Kroll, J. F., \& Stewart, E. (1994). Category interference in translation and picture naming: Evidence for asymmetric connections between bilingual memory representations. Journal of Memory and Language, 33, 149-174.

Kubus, O., Villwock, A., Morford, J. P., \& Rathmann, C. (2014). Word recognition in deaf readers: Cross-language activation of German Sign Language and German. Applied Psycholinguistics, 1-24.

Kuperberg, G. R., Sitnikova, T., Caplan, D., \& Holcomb, P. J. (2003). Electrophysiological distinctions in processing conceptual relationships within simple sentences. Cognitive Brain Research, 17, 117-129.

Kutas, M., \& Federmeier, K. D. (2011). Thirty years and counting: finding meaning in the N400 component of the event-related brain potential (ERP). Annual Review of Psychology, 62(14), 1-47.

Kutas, M., \& Hillyard, S. A. (1980). Reading senseless sentences: Brain potentials reflect semantic incongruity. Science, 207, 203-205.

Kutas, M., \& Hillyard, S. A. (1983). Event-related brain potentials to grammatical errors and semantic anomalies. Memory \& Cognition, 11(5), 539-550.

Kutas, M., Hillyard, S. A., \& Gazzaniga, M. S. (1988). Processing of semantic anomaly by right and left hemispheres of commissurotomy patients. Brain, 111, 553-576. 
Kutas, M., Neville, H. J., \& Holcomb, P. J. (1987). A preliminary comparison of the N400 response to semantic anomalies during reading, listening and signing. In R. J. Ellingson, N. M. F. Murray, \& A. M. Halliday (Eds.), Electro 7 encephalography and Clinical Neurophysiology Supplement 39, The London Symposia (Vol. 39, pp. 325-330). Amsterdam: Elsevier.

Lau, E. F., Phillips, C., \& Poeppel, D. (2008). A cortical network for semantics: (de)constructing the N400. Nature Reviews Neuroscience, 9(12), 920933.

Lawrence, M. A. (2012). ez: Easy analysis and visualization of factorial experiments.

Lemhöfer, K., Dijkstra, T., \& Michel, M. (2004). Three languages, one ECHO: Cognate effects in trilingual word recognition. Language and Cognitive Processes, 19(5), 585-611.

Leonard, M. K., Ferjan Ramirez, N., Torres, C., Travis, K. E., Hatrak, M., Mayberry, R. I., \& Halgren, E. (2012). Signed words in the congenitally deaf evoke typical late lexicosemantic responses with no early visual responses in left superior temporal cortex. The Journal of Neuroscience, 32(28), 9700-9705.

Leuninger, H., Hohenberger, A., Waleschkowski, E., Menges, E., \& Happ, D. (2004). The impact of modality on language production: Evidence from slips of the tongue and hand. In T. Pechmann \& C. Habel (Eds.), Multidisciplinary approaches to language production (pp. 219-277). Berlin: Mouton de Gruyter.

Liddell, S. (1990). Four Functions of a Locus: Re-examining the Structure of Space in ASL. In C. Lucas (Ed.), Sign language research: Theoretical issues (pp. 176-198). Washington, DC: Gallaudet University Press.

Liddell, S. (1995). Real, surrogate, and soken space: Grammatical consequences in ASL. In K. Emmorey \& J. Reilly (Eds.), Language, gesture, and space (pp. 19-42). Hillsdale, NJ: Lawrence Erlbaum.

Liddell, S. (2000). Indicating verbs and pronouns: Pointing away from agreement. In K. Emmorey \& H. Lane (Eds.), The signs of language revisited: An anthology to honor Ursula Bellugi and Edward Klima (pp. 303-320). Mahwah, NJ: Lawrence Erlbaum.

Liddell, S. (2003). Grammar, gesture and meaning in American Sign Language. Cambridge: Cambridge University Press.

Liddell, S., \& Johnson, R. E. (1989). American Sign Language: the phonological base. Sign Language Studies, 64, 195-277.

Lillo-Martin, D. (2012). Utterance reports and constructed action. In R. Pfau, M. Steinbach, \& B. Woll (Eds.), Sign Language. An international handbook (HSK) (pp. 365-387). Berlin, Boston: De Gruyter Mouton. 
Lillo-Martin, D., \& Klima, E. (1990). Pointing out differences: ASL pronouns in syntactic theory. In S. Fischer \& P. Siple (Eds.), Theoretical issues in sign language research, Vol.1: Linguistics (pp. 191-210). Chicago: University of Chicago Press.

Lillo-Martin, D., \& Meier, R. P. (2011). On the linguistic status of "agreement" in sign languages. Theoretical Linguistics, 37(3-4), 95-142.

MacSweeney, M., Campbell, R., Woll, B., Giampietro, V., David, A. S., McGuire, P. K., ... Brammer, M. J. (2004). Dissociating linguistic and nonlinguistic gestural communication in the brain. NeuroImage, 22(4), 1605-1618.

MacSweeney, M., Goswami, U., \& Neville, H. (2013). The neurobiology of rhyme judgment by deaf and hearing adults: An ERP study. Journal of Cognitive Neuroscience, 25(7), 1037-1048.

MacSweeney, M., Waters, D., Brammer, M. J., Woll, B., \& Goswami, U. (2008). Phonological processing in deaf signers and the impact of age of first language acquisition. NeuroImage, 40(3), 1369-1379.

MacSweeney, M., Woll, B., Campbell, R., McGuire, P. K., David, A. S., Williams, S. C. R., ... Brammer, M. J. (2002). Neural systems underlying British Sign Language and audio-visual English processing in native users. Brain, $125,1583-1593$.

Malaia, E., \& Wilbur, R. B. (2010). Early acquisition of sign language: What neuroimaging data tell us. Sign Language \& Linguistics, 13(2), 183-199.

Marentette, P. F., \& Mayberry, R. I. (2000). Principles for an emerging phonological system: A case study of early ASL acquisition. In C. Ghamberlain, J. P. Morford, \& R. I. Mayberry (Eds.), Language acquisition by eye (pp. 71-90). Mahwah, NJ: Lawrence Erlbaum.

Marian, V., Spivey, M., \& Hirsch, J. (2003). Shared and separate systems in bilingual language processing: Converging evidence from eyetracking and brain imaging. Brain and Language, 86(1), 70-82.

Marshall, J., Atkinson, J., Woll, B., \& Thacker, A. (2005). Aphasia in a bilingual user of British S Sign Language and English: Effects of cross-linguistic cues. Cognitive Neuropsychology, 22(6), 719-36.

Mathur, G., \& Rathmann, C. (2012). Verb agreement. In R. Pfau, M. Steinbach, \& B. Woll (Eds.), Sign Language. An international handbook (HSK) (pp. 136-157). Berlin, Boston: De Gruyter Mouton.

Mayberry, R. I. (2007). When timing is everything: Age of first-language acquisition effects on second-language learning. Applied Psycholinguistics, 28(03), 537-549.

Mayberry, R. I., Chen, J.-K., Witcher, P., \& Klein, D. (2011). Age of acquisition effects on the functional organization of language in the adult brain. Brain and Language, 119(1), 16-29. 
McBurney, S. L. (2012). History of sign languages and sign language linguistics. In R. Pfau, M. Steinbach, \& B. Woll (Eds.), Sign Language. An international handbook (HSK) (pp. 909-948). Berlin, Boston: De Gruyter Mouton.

McClelland, J. L., \& Elman, J. L. (1986). The TRACE model of speech perception. Cognitive Psychology, 18(1), 1-86.

McClelland, J. L., \& Rumelhart, D. E. (1981). An interactive activation model of context effects in letter perception: Part 1. An account of basic findings. Psychological Review, 88, 375-407.

McGurk, H., \& MacDonald, J. (1976). Hearing lips and seeing voices. Nature, 264, 746-748.

Meier, R. P. (1990). Person deixis in American Sign Language. In S. Fischer \& P. Siple (Eds.), Theoretical issues in sign language research, Vol.1: Linguistics (pp. 175-190). Chicago: University of Chicago Press.

Meier, R. P. (2002). Why different, why the same? Explaining effects and noneffects of modality upon linguistic structure in sign and speech. In R. P. Meier, K. Cormier, \& D. Quinto-Pozos (Eds.), Modality and structure in signed and spoken languages (pp. 1-25). Cambridge: Cambridge University Press.

Meier, R. P., Cormier, K., \& Quinto-Pozos, D. (Eds.). (2002). Modality and structure in signed and spoken languages. Cambridge: Cambridge University Press.

Meir, I. (1998). Thematic structure and verb agreement in Israeli Sign Language. Hebrew University of Jerusalem.

Meir, I. (2002). A cross-modality perspective on verb agreement. Natural Language \& Linguistic Theory, 20, 413-450.

Meir, I. (2012). Word classes and word formation. In R. Pfau, M. Steinbach, \& B. Woll (Eds.), Sign Language. An international handbook (HSK) (pp. 77111). Berlin, Boston: De Gruyter Mouton.

Molinaro, N., Barber, H. A., \& Carreiras, M. (2011). Grammatical agreement processing in reading: ERP findings and future directions. Cortex, 47(8), 908-930.

Morford, J. P., Wilkinson, E., Villwock, A., Piñar, P., \& Kroll, J. F. (2011). When deaf signers read English: Do written words activate their sign translations? Cognition, 118(2), 286-292.

Morgan, G., Barrett-Jones, S., \& Stoneham, H. (2007). The first signs of language: phonological development in British Sign Language. Applied Psycholinguistics, 28(1), 3-22. 
Neidle, C., Kegl, J., MacLaughlin, D., Bahan, B., \& Lee, R. (2000). The syntax of American Sign Language: Functional categories and hierarchical structure. Cambridge, MA: MIT Press.

Neville, H. J., Coffey, S. A., Lawson, D. S., Fischer, A., Emmorey, K., \& Bellugi, U. (1997). Neural systems mediating American Sign Language: effects of sensory experience and age of acquisition. Brain and Language, 57, 285308.

Neville, H. J., \& Lawson, D. S. (1987). Attention to central and peripheral visual space in a movement detection task: An event-related potential and behavioral study. II. Congenitally deaf adults. Brain Research, 405(2), 268-283.

Nieuwenhuis, S., Aston-Jones, G., \& Cohen, J. D. (2005). Decision making, the P3, and the locus coerulus-norepinephrine system. Psychological Bulletin, 131, 510-532.

Nieuwland, M. S., \& van Berkum, J. J. A. (2006). Individual differences and contextual bias in pronoun resolution: evidence from ERPs. Brain Research, 1118(1), 155-67.

Nieuwland, M. S., \& van Berkum, J. J. A. (2008). The Neurocognition of Referential Ambiguity in Language Comprehension. Language and Linguistics Compass, 2(4), 603-630.

Norris, D. (1994). Shortlist: a connectionist model of continuous speech recognition. Cognition, 52(3), 189-234.

Orfanidou, E., Adam, R., McQueen, J. M., \& Morgan, G. (2009). Making sense of nonsense in British Sign Language (BSL): The contribution of different phonological parameters to sign recognition. Memory \& Cognition, 37(3), 302-315.

Ormel, E., Hermans, D., Knoors, H., \& Verhoeven, L. (2012). Cross-language effects in written word recognition: The case of bilingual deaf children. Bilingualism: Language and Cognition, 15(02), 288-303.

Osterhout, L., \& Mobley, L. (1995). Event-related brain potentials elicited by failure to agree. Journal of Memory and Language, 34, 739-773.

Otten, M., Nieuwland, M. S., \& van Berkum, J. (2007). Great expectations: specific lexical anticipation influences the processing of spoken language. BMC Neuroscience, 8:89.

Oz, H. (2007). The neural correlates of syntactic processing in Israeli Sign Language: An ERP study. Ben Gurion University.

Padden, C. (1983). Interaction of morphology and syntax in American Sign Language. University of California, San Diego. 
Padden, C., \& Perlmutter, D. (1987). American Sign Language and the architecture of phonological theory. Natural Language \& Linguistic Theory, 5, 335-375.

Pfau, R., \& Quer, J. (2010). Nonmanuals: their prosodic and grammatical roles. In D. Brentari (Ed.), Sign Languages (pp. 381-402). Cambridge: Cambridge University Press.

Pfau, R., \& Steinbach, M. (2006). Modality-independent and modality-specific aspects of grammaticalization in sign languages. Linguistics in Potsdam, 24, 5-98.

Pfau, R., \& Steinbach, M. (2011). Grammaticalization in sign languages. In B. Heine \& H. Narrog (Eds.), The Oxford handbook of grammaticalization (pp. 681-693). Oxford: Oxford University Press.

Pickering, M. J., \& Garrod, S. (2007). Do people use language production to make predictions during comprehension? Trends in Cognitive Sciences, 11, 105-110.

Plaza-Pust, C., \& Weinmeister, K. (2008). Bilingual acquisition of German Sign Language and written German: Developmental asynchronies and language contact. In R. M. de Quadros (Ed.), Sign Languages: spinning and unraveling the past, present and future. TISLR9, forty five papers and three posters from the 9th. Theoretical Issues in Sign Language Research Conference, Florianópolis, Brazil, December 2006 (pp. 497-529). Petrópolis, RJ, Brazil: Editora Arara Azul.

Poizner, H., Klima, E. S., \& Bellugi, U. (1987). What the hands reveal about the brain. Cambridge, MA: MIT Press.

Polich, J. (2007). Updating P300: An integrative theory of P3a and P3b. Clinical Neurophysiology, 118(10), 2128-2148.

Praamstra, P., Meyer, A. S., \& Levelt, W. J. M. (1994). Neurophysiological manifestations of phonological processing: Latency variation of a negative ERP component timelocked to phonological mismatch. Journal of Cognitive Neuroscience, 6(3), 204-219.

Pyers, J. E., \& Emmorey, K. (2008). The face of bimodal bilingualism: Grammatical markers in American Sign Language are produced when bilinguals speak to English monolinguals. Psychological Science, 19(6), 531-536.

Quadros, R. M. de, \& Quer, J. (2008). Back to back(wards) and moving on: on agreement, auxiliaries and verb classes in sign languages. In R. M. de Quadros (Ed.), Sign Languages: spinning and unraveling the past, present and future. TISLR9, forty five papers and three posters from the 9th. Theoretical Issues in Sign Language Research Conference, Florianópolis, Brazil, December 2006 (pp. 530-551). Petrópolis, RJ, Brazil: Editora Arara Azul. 
Quer, J. (2011). When agreeing to disagree is not enough: Further arguments for the linguistic status of sign language agreement. Theoretical Linguistics, 4, 189-196.

Radeau, M., Morais, J., \& Segui, J. (1995). Phonological priming between monosyllabic spoken words. Journal of Experimental Psychology: Human Perception and Performance, 21, 1297-1311.

Rathmann, C. (2000). The optionality of agreement phrase: Evidence from signed languages. The University of Texas at Austin.

Rathmann, C., \& Mathur, G. (2002). Is verb agreement the same crossmodally? In R. Meier, K. Cormier, \& D. Quinto-Pozos (Eds.), Modality and structure in signed and spoken languages (pp. 370-404). Cambridge: Cambridge University Press.

Rathmann, C., \& Mathur, G. (2008). Verb agreement as a linguistic innovation in signed languages. In J. Quer (Ed.), Signs of the time: Selected papers from TISLR 2004 (pp. 191-216). Hamburg: Signum.

Rauschecker, J. P., \& Scott, S. K. (2009). Maps and streams in the auditory cortex: nonhuman primates illuminate human speech processing. Nature Neuroscience, 12(6), 718-724.

Roehm, D., Bornkessel-Schlesewsky, I., Rösler, F., \& Schlesewsky, M. (2007). To predict or not to predict: Influences of task and strategy on the processing of semantic relations. Journal of Cognitive Neuroscience, 19(8), 1259-1274.

Rugg, M. D. (1990). Event-related brain potentials dissociate repetition effects of high- and low-frequeney words. Memory \& Cognition, 18, 367-379.

Sandler, W. (1989). Phonological representation of the sign: linearity and nonlinearity in sign language phonology. Dodrecht: Foris.

Sandler, W., \& Lillo-Martin, D. (2006). Sign language and linguistic universals. Cambridge: Cambridge University Press.

Sanford, A. J., Leuthold, H., Bohan, J., \& Sanford, A. J. S. (2011). Anomalies at the borderline of awareness: an ERP study. Journal of Cognitive Neuroscience, 93, 514-523.

Sassenhagen, J., Schlesewsky, M., \& Bornkessel-Schlesewsky, I. (2014). The P600-as-P3 hypothesis revisited: Single-trial analyses reveal that the late EEG positivity following linguistically deviant material is reaction time aligned. Brain and Language, 137, 29-39.

Schwager, W., \& Zeshan, U. (2008). Word classes in sign languages: Criteria and classifications. Studies in Language, 32(3), 509-545.

Shook, A., \& Marian, V. (2010). Language processing across modalities: Insights from bimodal bilingualism. Cognitive Sciences, 5(1), 57-98. 
Shook, A., \& Marian, V. (2012). Bimodal bilinguals co-activate both languages during spoken comprehension. Cognition, 124(3), 314-324.

Skotara, N., Kügow, M., Salden, U., Hänel-Faulhaber, B., \& Röder, B. (2011). ERP correlates of intramodal and crossmodal L2 acquisition. BMC Neuroscience, 12(1), 48.

Slowiaczek, L. M., Nusbaum, H. C., \& Pisoni, D. B. (1987). Phonological priming in auditory word recognition. Journal of Experimental Psychology: Learning, Memory, and Cognition, 13, 64-75.

Squires, N. K., Squires, K. C., \& Hillyard, S. A. (1975). Two varieties of longlatency positive waves evoked by unpredictable auditory stimuli in man. Electroencephalography and Clinical Neurophysiology, 38(4), 387401.

Steinbach, M., \& Onea, E. (2015). A DRT Analysis of Discourse Referents and Anaphora Resolution in Sign Language. Journal of Semantics, 1-30.

Steinbach, M., \& Pfau, R. (2007). Grammaticalization of auxiliaries in sign languages. In P. Perniss, R. Pfau, \& M. Steinbach (Eds.), Trends in linguistics: Visible Variation. Comparative studies on sign language structure. (pp. 303-339). Berlin: Mouton de Gruyter.

Stokoe, W. C. (1960). Sign language structure: An outline of the visual communication systems of the American Deaf. In Studies in Linguistics: Occasional papers (No. 8). Buffalo: University of Buffalo Press.

Stokoe, W. C., Casterline, D. C., \& Cronberg, C. G. (1965). A dictionary of American Sign Language on linguistic principles. Washington DC: Gallaudet University Press.

Stroud, C., \& Phillips, C. (2012). Examining the evidence for an independent semantic analyzer: an ERP study in Spanish. Brain and Language, 120, 108-126.

Supalla, T. (1982). Structure and acquisition of verbs of motion and location in American Sign Language. University of California, San Diego.

Supalla, T. (1986). The classifier system in American Sign Language. In C. Craig (Ed.), Noun classes and categorization (pp. 181-214). Amsterdam: John Benjamins.

Sutton, S., Braren, M., Zubin, J., \& John, E. (1965). Evoked potential correlates of stimulus uncertainty. Science, 150, 1187-1188.

Sutton-Spence, R., \& Day, L. (2001). Mouthings and mouth gestures in British Sign Language (BSL). In P. B. Braem \& R. Sutton-Spence (Eds.), The hands are the head of the mouth: The mouth as articulator in sign languages (pp. 69-85). Hamburg: Signum. 
Sutton-Spence, R., \& Woll, B. (1999). The linguistics of British Sign Language. An Introduction. Cambridge: Cambridge University Press.

Team, R.D.C. (2012). R: A language and environment for statistical computing. Vienna, Austria.

Thierry, G., \& Wu, Y. J. (2007). Brain potentials reveal unconscious translation during foreign-language comprehension. Proceedings of the National Academy of Sciences of the United States of America (PNAS), 104(30), 12530-12535.

Thompson, R. L. (2006). Eye gaze in American Sign Language: Linguistic functions for verbs and pronouns. University of California, San Diego.

Thompson, R., Emmorey, K., \& Gollan, T.H. (2005). "Tip of the fingers" experiences by deaf signers: insights into the organization of a signbased lexicon. Psychological Science, 16(11), 856-860.

Thompson, R. L., Emmorey, K., \& Kluender, R. (2009). Learning to look: The acquisition of eye gaze agreement during the production of ASL verbs. Bilingualism: Language and Cognition, 12(04), 393-409.

Treiman, R., \& Hirsh-Pasek, K. (1983). Silent reading: Insights from secondgeneration deaf readers. Cognitive Psychology, 15, 39-65.

Van Berkum, J.J.A., Brown, C.M., \& Hagoort, P. (1999a). Early referential context effects in sentence processing: evidence from event-related brain potentials. Journal of Memory and Language, 41, 147-182.

Van Berkum, J.J.A., Brown, C.M., \& Hagoort, P. (1999b). When does gender constrain parsing? Evidence from ERPs. Journal of Psycholinguistic Research, 28(5), 555-571.

Van Berkum, J.J.A., Brown, C. M., Hagoort, P., \& Zwitserlood, P. (2003). Eventrelated brain potentials reflect discourse-referential ambiguity in spoken language comprehension. Psychophysiology, 40(2), 235-248.

Van Berkum, J.J.A., Brown, C.M., Zwitserlood, P., Kooijman, V., \& Hagoort, P. (2005). Anticipating upcoming words in discourse: evidence from ERPs and reading times. Journal of Experimental Psychology: Learning, Memory, and Cognition, 31(3), 443-467.

Van Berkum, J.J.A., Hagoort, P., \& Brown, C.M. (1999). Semantic integration in sentences and discourse: Evidence from the N400. Journal of Cognitive Neuroscience, 11(6), 657-671.

Van Berkum, J.J.A., Zwitserlood, P., Bastiaansen, M.C.M., Brown, C. M., \& Hagoort, P. (2004). So who's "he" anyway? Differential ERP and ERSP effects of referential success, ambiguity and failure during spoken language comprehension. In Annual meeting of the Cognitive Neuroscience Society (CNS-2004), San Francisco, April 18-20. 
Van Berkum, J.J.A., Koornneef, A. W., Otten, M., \& Nieuwland, M. S. (2007). Establishing reference in language comprehension: an electrophysiological perspective. Brain Research, 1146, 158-171.

Van den Brink, D., Brown, C. M., \& Hagoort, P. (2001). Electrophysiological evidence for early contextual influences during spoken-word recognition: N200 versus $\mathrm{N} 400$ effects. Journal of Cognitive Neuroscience, 13:7, 967-985.

Van Wijnendaele, I., \& Brysbaert, M. (2002). Visual word recognition in bilinguals: Phonological priming from the second to the first language. Journal of Experimental Psychology: Human Perception and Performance, 28(3), 616-627.

Van Petten, C. (1995). Words and sentences : Event-related brain potential measures. Psychophysiology, 32, 511-525.

Van Petten, C., \& Kutas, M. (1990). Interactions between sentence context and word frequency in event-related potentials. Memory \& Cognition, 18, 380-393.

Verleger, R. (1988). Event-related potentials and cognition: A critique of the context updating hypothesis and an alternative interpretation of P3. Behavioral and Brain Sciences, 11, 343-427.

Verleger, R. (1998). Toward an integration of P3 research with cognitive neuroscience. Behavioral and Brain Sciences, 21, 149-168.

Wicha, N.Y.Y., Moreno, E. M., \& Kutas, M. (2004). Anticipating words and their gender: an event-related brain potential study of semantc integration, gender expectancy, and gender agreement in Spanish sentence reading. Journal of Cognitive Neuroscience, 16, 1271-1288.

Wilbur, R.B. (2000). Phonological and prosodic layering of nonmanuals in American Sign Language. In K. Emmorey \& H. Lane (Eds.), The signs of language revisited: An anthology to honor Ursula Bellugi and Edward Klima (pp. 213-241). Mahwah, NJ: Lawrence Erlbaum Associates.

Wilbur, R. B. (2013). The point of agreement: Changing how we think about sign language, gesture, and agreement. Sign Language \& Linguistics, 16(2), 221-258.

Woll, B. (2001). The sign that dares to speak its name: Echo phonology in British Sign Language (BSL). In P. B. Braem \& R. Sutton-Spence (Eds.), The hands are the head of the mouth: The mouth as articulator in sign languages (pp. 87-98). Hamburg: Signum.

Wu, Y. J., \& Thierry, G. (2010). Chinese-English bilinguals reading English hear Chinese. The Journal of Neuroscience, 30(22), 7646-7651.

Zachau, S., Korpilahti, P., Hämäläinen, J. a, Ervast, L., Heinänen, K., Suominen, K., ... Leppänen, P. H. T. (2014). Electrophysiological correlates of cross- 
linguistic semantic integration in hearing signers: N400 and LPC. Neuropsychologia, 59, 57-73.

Zwitserlood, I. (2003). Classifying hand configurations in Nederlandse Gebarentaal (Sign Language of the Netherlands). Utrecht: LOT.

Zwitserlood, I. (2012). Classifiers. In R. Pfau, M. Steinbach, \& B. Woll (Eds.), Sign Language. An international handbook (HSK) (pp. 158-186). Berlin, Boston: De Gruyter Mouton.

Zwitserlood, I., \& Gijn, I. van. (2006). Agreement phenomena in Sign Language of the Netherlands. In P. Ackema, P. Brandt, M. Schoorlemmer, \& F. Weerman (Eds.), Arguments and Agreement (pp. 195-229). Oxford: Oxford University Press. 


\section{Appendix A - Stimulus materials}

\section{Chapter 1: Complete list of stimulus sentences}

\begin{tabular}{|c|c|c|}
\hline Verb & Condition & Handshape/Orientation \\
\hline \multicolumn{3}{|c|}{ Action verbs: } \\
\hline \multirow[t]{10}{*}{ MOVE $_{\text {FLAT OBJ }}$} & expected & \\
\hline & BERLIN IX CAR SLOWLY MOVE & B-hand; palm down \\
\hline & LONDON IX BUS DOUBLE\#DECKER MOVE & B-hand; palm down \\
\hline & STREET IX BOY SKATEBOARD MOVE & B-hand; palm down \\
\hline & HAMBURG IX RIVER STEAMBOAT MOVE & B-hand; palm down \\
\hline & unexpected & \\
\hline & LIBRARY IX TABLE BOOK MOVE & B-hand; palm down \\
\hline & WAITING\#ROOM IX TABLE NEWSPAPER MOVE & B-hand; palm down \\
\hline & ARCHIVE IX SHELF PAPER MOVE & B-hand; palm down \\
\hline & OFFICE IX TABLE LAPTOP MOVE & B-hand; palm down \\
\hline \multirow[t]{9}{*}{ ROLL } & expected & \\
\hline & STREET IX WIND BOTTLE ROLL & G-hand; $2 \mathrm{~h}$ altern.; palm down \\
\hline & SOCCER\#FIELD IX BALL GOOD ROLL & G-hand; $2 \mathrm{~h}$ altern.; palm down \\
\hline & HIGHWAY IX ACCIDENT CAR ROLL & G-hand; $2 \mathrm{~h}$ altern.; palm down \\
\hline & $\begin{array}{l}\text { HILL IX GRASSLAND CHILDREN ROLL } \\
\text { unexpected }\end{array}$ & G-hand; $2 \mathrm{~h}$ altern.; palm down \\
\hline & UNIVERSITY IX AUDITORIUM PROFESSOR ROLL & G-hand; $2 \mathrm{~h}$ altern.; palm down \\
\hline & KITCHEN IX BENCH GRANDMA ROLL & G-hand; $2 \mathrm{~h}$ altern.; palm down \\
\hline & RESTAURANT IX MAN BEER ROLL & G-hand; $2 \mathrm{~h}$ altern.; palm down \\
\hline & HOSPITAL IX DOCTOR APPENDIX ROLL & G-hand; $2 \mathrm{~h}$ altern.; palm down \\
\hline
\end{tabular}

$\begin{array}{ll}\text { JUMP } & \text { expected } \\ & \text { LAKE IX GRASS FROG JUMP } \\ & \text { PARK IX PATH SQUIRREL JUMP } \\ & \text { WOODS IX PATH RABBIT JUMP } \\ & \text { ZOO IX LAWN KANGAROO JUMP } \\ & \text { unexpected } \\ & \text { RIVER IX CROCODILE MEAT JUMP } \\ & \text { PADDOCK IX COW HEY JUMP } \\ & \text { ZOO IX RHINO BREAD JUMP } \\ & \text { FARM IX MOUSE CHEESE JUMP } \\ & \text { eXPected } \\ \text { EVERY MONTH MOTHER HAIRDRESSER GO } \\ \text { WEEKEND NEXT I CINEMA GO } \\ \text { TOMORROW CHILD SCHOOL GO } \\ \text { SUNDAY GRANDMA CHURCH GO } \\ \text { UneXPected } \\ \text { TABLE IX BALL-PEN BLUE GO } \\ \text { GARDEN IX ROSE RED GO } \\ \text { CAFÉ IX GRANDMA TEA GO } \\ \text { CHRISTMAS\#MARKET IX FATHER WINE GO }\end{array}$

3-hand-bend; palm down 3-hand-bend; palm down 3-hand-bend; palm down 3-hand-bend; palm down

3-hand-bend; palm down 3-hand-bend; palm down 3-hand-bend; palm down 3-hand-bend; palm down

G-hand; finger up, palm in G-hand; finger up, palm in G-hand; finger up, palm in G-hand; finger up, palm in

G-hand; finger up, palm in G-hand; finger up, palm in G-hand; finger up, palm in G-hand; finger up, palm in 
CLIMB

expected

ZOO IX TREE MONKEY CLIMB

PLAYGROUND IX FRAME CHILD CLIMB

GARDEN IX TREE CAT CLIMB

SPAIN IX WALL LIZARD CLIMB

unexpected

AFRICA IX RIVER CROCODILE CLIMB

COUCH IX CUSHION SOFT CAT CLIMB

ZOO IX KANGAROO LAWN CLIMB

FARM IX MUD PIG CLIMB

\section{Non-action verbs:}

LAY $_{\text {HUMAN }}$

SIT

STAND $_{\text {HUMAN }}$

STAND expected

VACATION IX BEACH MAN LAZY LAY

BALCONY IX WOMAN SUN LAY

EVENING BED IX CHILD TIRED LAY

HOSPITAL IX ROOM GIRL INJURED LAY

unexpected

CLIMBING\#WALL DANGEROUS IX MAN LAY

WEDDING IX FATHER JOKE LAY

CAFÉ IX GRANDMA CAKE LAY

LONDON IX CAR QUEEN LAY

expected

HOSPITAL IX WAITING\#ROOM MAN SIT

SCHOOL IX CHILD BENCH SIT

EVENING CINEMA IX BOY FIRST ROW SIT

TRAM IX FOLDING\#SEET MAN SIT

unexpected

COMPUTER SHOP IX MAN LAPTOP NEW SIT H\&M IX BOY SHIRT MODERN SIT

BAKERY IX GRANDPA BUN SIT

JEWELRY\#SHOP IX WOMAN RING SIT

\section{expected}

MORNING BUS\#STOP IX MAN STAND

SHOP NEW IX QUEUE WOMAN STAND

DISCO ENTRANCE IX DOORMAN STAND

CINEMA ENTRANCE IX MAN YOUNG STAND

unexpected

MORNING WOMAN TEA STAND

KINDERGARDEN IX CHILDREN BALL STAND

CHRISTMAS FAMILY GOOSE\#MEAT STAND

BATHROOM IX CRAFTSMAN PIPE STAND

expected

SCHOOL IX SHELF BOOK SASS-CL STAND

BERLIN IX WALL SASS-CL STAND

LIVING\#ROOM IX CUPBOARD DVD SASS-CL STAND

OFFICE IX SHELF DICTIONARY STAND

unexpected

SCHOOL IX PRINTER PAPER SASS-CL STAND

DRAWING\#TABLE IX RULER SASS-CL STAND

PHOTO\#ALBUM IX PICTUER OLD STAND

PARIS IX MUSEUM PICASSO PAINTING STAND 5-hand-bend; $2 \mathrm{~h}$ altern., palm front

5-hand-bend; $2 \mathrm{~h}$ altern., palm front

5-hand-bend; $2 \mathrm{~h}$ altern., palm front

5-hand-bend; $2 \mathrm{~h}$ altern., palm front

5-hand-bend; $2 \mathrm{~h}$ altern., palm front 5-hand-bend; $2 \mathrm{~h}$ altern., palm front 5 -hand-bend; $2 \mathrm{~h}$ altern., palm front 5-hand-bend; $2 \mathrm{~h}$ altern., palm front

V-hand; finger in, palm up V-hand; finger in, palm up V-hand; finger in, palm up V-hand; finger in, palm up

V-hand; finger in, palm up V-hand; finger in, palm up V-hand; finger in, palm up V-hand; finger in, palm up

V-hand-bend; palm in V-hand-bend; palm in V-hand-bend; palm in V-hand-bend; palm in

V-hand-bend; palm in V-hand-bend; palm in V-hand-bend; palm in V-hand-bend; palm in

V-hand; finger down V-hand; finger down V-hand; finger down V-hand; finger down

V-hand; finger down $V$-hand; finger down V-hand; finger down V-hand; finger down

B-hand; finger up, palm in B-hand; finger up, palm in B-hand; finger up, palm in $B$-hand; finger up, palm in

B-hand; finger up, palm in B-hand; finger up, palm in B-hand; finger up, palm in B-hand; finger up, palm in 
FALL-

DOWN $_{\text {HUMAN }}$ expected

ROOF IX CONSTRUCTION-WORKER DRUNKEN FALL-DOWN TABLE DIAPER-CHANGING IX BABY TWITCHY FALL-DOWN CIRCUS IX TIGHTROPE-ARTIST LIGHT-HEADED FALL-DOWN CLIMBING\#WALL DANGEROUS IX MAN FALL-DOWN unexpected

LAWN IX WOMAN FLOWER FALL-DOWN

BEACH IX MAN LAZY FALL-DOWN

OFFICE IX SCIENTIST BOOK FALL-DOWN CINEMA COUNTER IX BOY TICKET FALL-DOWN
V-hand; finger in, palm down V-hand; finger in, palm down V-hand; finger in, palm down V-hand; finger in, palm down

V-hand; finger in, palm down V-hand; finger in, palm down V-hand; finger in, palm down V-hand; finger in, palm down 
Chapter 2: Complete list of stimulus sentences; DGS priming

\begin{tabular}{|c|c|c|}
\hline prime / target & critical and control sentences & phon. \\
\hline DOCTOR / MINUTE & $\begin{array}{l}\text { YESTERDAY ACCIDENT DOCOTOR FIVE MINUTE LATE COME } \\
\text { YESTERDAY ACCIDENT POLICE FIVE MINUTE LATE COME }\end{array}$ & loc \\
\hline NOON / WATER & $\begin{array}{l}\text { DAILY NOON IX } 1 \text { WATER DRINK SHOULD } \\
\text { DAILY EVENING IX } 1 \text { WATER DRINK SHOULD }\end{array}$ & hs \\
\hline ORANGE / CHAOS & $\begin{array}{l}\text { SUPERMARKET IX ORANGE SAME CHAOS LOOKS } \\
\text { SUPERMARKET IX APPLE SAME CHAOS LOOKS }\end{array}$ & mov \\
\hline ROOM / FAMILY & $\begin{array}{l}\text { NEXT WEEK ROOM IX FAMILY PARTY ORGANIZE } \\
\text { NEXT WEEK RESTAURANT IX FAMILY PARTY ORGANIZE }\end{array}$ & hs \\
\hline ACCEPTANCE / REGISTRATION & $\begin{array}{l}\text { TALK TOPIC DGS POSS ACCEPTANCE IX REGISTRATION IMPORTANT } \\
\text { TALK TOPIC DGS POSS GRAMMAR IX REGISTRATION IMPORTANT }\end{array}$ & hs \\
\hline TREE / TALK & $\begin{array}{l}\text { TOPIC TREE IX }{ }_{1} \text { TALK PREPARE MUST } \\
\text { TOPIC ANIMAL IX } 1 \text { TALK PREPARE MUST }\end{array}$ & mov \\
\hline STORE / ANIMAL & $\begin{array}{l}\text { USUALLY STORE IX ANIMAL ALLOWED-NEG VISIT } \\
\text { USUALLY CHURCH IX ANIMAL ALLOWED-NEG VISIT }\end{array}$ & mov \\
\hline CULTURE / FLOWER & $\begin{array}{l}\text { INDIEN POSS CULTURE IX FLOWER IMPORTANT } \\
\text { INDIEN POSS RELIGION IX FLOWER IMPORTANT }\end{array}$ & mov \\
\hline MEDICINE / SCIENCE & $\begin{array}{l}\text { CHINA IX MEDICINE SAME SCIENCE OLD } \\
\text { CHINA IX ART SAME SCIENCE OLD }\end{array}$ & loc \\
\hline HIKE / PAIN & $\begin{array}{l}\text { YESTERDAY HIKE IX }{ }_{1} \text { PAIN SHOULDER } \\
\text { YESTERDAY SPORT IX } 1 \text { PAIN SHOULDER }\end{array}$ & mov \\
\hline WORRIES / DREAM & $\begin{array}{l}\text { MANY WORRIES OFTEN DREAM BAD INFLUENCE } \\
\text { MANY STRESS OFTEN DREAM BAD INFLUENCE }\end{array}$ & hs \\
\hline BIRTHDAY / ADVANTAGE & $\begin{array}{l}\text { SUMMER BIRTHDAY IX ADVANTAGE REASON WEATHER NICE } \\
\text { SUMMER WEDDING IX ADVANTAGE REASON WEATHER NICE }\end{array}$ & mov \\
\hline WORK / STONE & $\begin{array}{l}\text { MY GRANDPA POSS WORK IX STONE HAMMER } \text { HL }_{\text {H }} \\
\text { MY GRANDPA POSS HOBBY IX STONE HAMMER } \\
\text { CL }\end{array}$ & mov \\
\hline INTERVIEW / DIALOGUE & $\begin{array}{l}\text { TELEVISION INTERVIEW IX } 1 \text { DIALOGUE DIFFICULT UNDERSTAND } \\
\text { TELEVISION MOVIE IX }{ }_{1} \text { DIALOGUE DIFFICULT UNDERSTAND }\end{array}$ & hs \\
\hline SOCIETY / PARTY & $\begin{array}{l}\text { DEAF SOCIETY OFTEN PARTY ORGANIZE } \\
\text { DEAF COMMUNITY OFTEN PARTY ORGANIZE }\end{array}$ & or \\
\hline FUTURE / CRITIQUE & $\begin{array}{l}\text { IX }_{1} \text { THINK FUTURE IX } \mathrm{X}_{\mathrm{pl}} \text { CRITIQUE WITHSTAND MUST } \\
\text { IX }_{1} \text { THINK 1-YEAR IX } \mathrm{X}_{\mathrm{pl}} \text { CRITIQUE WITHSTAND MUST }\end{array}$ & hs \\
\hline MATCH / DISCUSSION & $\begin{array}{l}\text { TOPIC SOCCER MATCH IX DISCUSSION OFTEN LONG } \\
\text { TOPIC SOCCER CHAMPIONSHIP IX DISCUSSION OFTEN LONG }\end{array}$ & hs \\
\hline FUN / MISTAKE & $\begin{array}{l}\text { TODAY IX }_{1} \text { FUN } 10 \text { MISTAKE FIND } \\
\text { TODAY IX }_{1} \text { SUCCESS } 10 \text { MISTAKE FIND }\end{array}$ & hs \\
\hline LONDON / BATHROOM & $\begin{array}{l}\text { IX } 1 \text { VACATION LONDON IX BATHROOM LOOK-FOR } \\
\text { IX } 1 \text { VACATION PARIS IX BATHROOM LOOK-FOR }\end{array}$ & hs \\
\hline
\end{tabular}


FEAR / PROBLEM

STATE / CONFERENCE

TRAINING / EXAMPLE

GRANDMA / PIG

HOBBY / ILLNESS

INJURY / METAL

RESTAURANT / DIALOGUE

PROOF / EXPENSES

PRACTICE / CAR

HILL / WOMAN

GRAVE / SHIP

MATHEMATICS / RAIN

WORK / UMBRELLA

TOWER / UMBRELLA

DAY / SENTENCE

DOG / LAUD

COFFEE / WORK

UMBRELLA / COFFEE

GRIEF / BATH

BANK / STORE

AGENCY / PRINTOUT
HEIGHT FEAR IX PROBLEM BIG FOR-ME MANY DEPT IX PROBLEM BIG FOR-ME

hs

THIS YEAR STATE IX CONFERENCE ORGANIZE THIS YEAR UNIVERSITY IX CONFERENCE ORGANIZE

hs

JOB TRAINING IX EXAMPLE MANY EXIST JOB SCHOOL IX EXAMPLE MANY EXIST

mov

PAST FARM IX MY GRANDMA OFTEN PIG FEED PAST FARM IX MY FATHER OFTEN PIG FEED

loc

hs DIFFERENT FRUIT IX $X_{\mathrm{pl}}$ ILLNESS PROTECT

JOINER POSS INJURY IX METAL CUT JOINER POSS INJURY IX SAW CUT

mov

WAITER RESTAURANT IX DIALOGUE SIGN CAN WAITER BAR IX DIALOGUE SIGN CAN

hs

TAX\#RETURN IX 1 PROOF FOR EXPENSES NEED TAX\#RETURN IX ${ }_{1}$ RECEIPT FOR EXPENSES NEED

hs

DRIVE\# LEARNER MUCH PRACTICE WITH CAR NEED DRIVE\# LEARNER MUCH EXPERIENCE WITH CAR NEED

mov

YESTERDAY BAVARIA HILL IX WOMAN STAND YESTERDAY BAVARIA LAWN IX WOMAN STAND

loc

CAPTAIN POSS GRAVE IF SHIP SINK CAPTAIN POSS PROBLEM IF SHIP SINK

loc

LAW MATHEMATICS SO RAIN ALWAYS FALL-STRAIGTH ${ }_{C L}$ LAW NATURE SO RAIN ALWAYS FALL-STRAIGTH ${ }_{C L}$

or

YESTERDAY IX 1 POSS 1 WORK IX UMBRELLA FORGET YESTERDAY IX ${ }_{1}$ POSS $_{1}$ SCHOOL IX UMBRELLA FORGET

mov

YESTERDAY IX 1 TOWER IX UMBRELLA FORGET YESTERDAY IX ${ }_{1}$ HOUSE IX UMBRELLA FORGET

hs

ABOUT EVERY DAY IX 1 SENTENCE ENGLISH WRITE ABOUT EVERY WEEK IX 1 SENTENCE ENGLISH WRITE

mov

MY DOG IX LAUD NEED

MY CAT IX LAUD NEED

loc

BRAZIL POSS COFFEE IX WORK PROCESS LONG BRAZIL POSS TOBACCO IX WORK PROCESS LONG

mov

IX 1 TERRACE UMBRELLA IX COFFEE DRINK

IX $_{1}$ TERRACE ROOF IX COFFEE DRINK

mov

LOVE GRIEF IX BATH WARM HELP

LOVE PROBLEM IX BATH WARM HELP

mov

STREET IX BANK NEXT STORE ALREADY CLOSE STREET IX SCHOOL NEXT STORE ALREADY CLOSE

mov

TOMORROW MAN AGENCY IX PRINTOUT PICK-UP TOMORROW MAN OFFICE IX PRINTOUT PICK-UP mov 
Chapter 2: Complete list of stimulus sentences; German priming

\begin{tabular}{|c|c|c|}
\hline & prime / target & critical and control sentences \\
\hline G: & Engel / Angel & MUSEUM IX PICTURE CONTENT TOPIC ANGEL WITH FISHING-ROD HANG \\
\hline E: & angel / fishing-rod & MUSEUM IX PICTURE CONTENT TOPIC MAN WITH FISHING-ROD HANG \\
\hline G: & Raum / Baum & ARCHITECT WISH ROOM IX TREE PLACE ${ }_{C L}$ \\
\hline E: & room / tree & ARCHITECT WISH HOUSE IX TREE PLACE ${ }_{\mathrm{CL}}$ \\
\hline G: & Wein / Bein & YESTERDAY RESTAURANT IX WINE IX ${ }_{1}$ LEG SPILL $_{C L}$ \\
\hline E: & wine / leg & YESTERDAY RESTAURANT IX CHAMPAGNE IX LEG SPILL $_{\mathrm{CL}}$ \\
\hline G: & Tier / Bier & USUALLY ANIMAL IX BEER DRINK LIKE-NEG \\
\hline E: & animal / beer & USUALLY CHILD IX BEER DRINK LIKE-NEG \\
\hline G: & Tuch / Buch & MAGUS PERSON CLOTH IX BOOK LAY-BENEATH ${ }_{\mathrm{CL}}$ \\
\hline E: & cloth / book & MAGUS PERSON TABLE IX BOOK LAY-BENEATH ${ }_{\mathrm{CL}}$ \\
\hline G: & Mutter / Butter & REFRIGERATOR POSS ${ }_{1}$ MOTHER IX BUTTER FETCH \\
\hline E: & mother / butter & REFRIGERATOR POSS 1 FATHER IX BUTTER FETCH \\
\hline G: & Dach / Schach & HOUSE POSS 3 ROOF LIKE CHESS PATTERN LOOK \\
\hline E: & roof / chess & HOUSE POSS 3 DOOR LIKE CHESS PATTERN LOOK \\
\hline G: & Kampf / Dampf & LAST-TIME BOX FIGHT IX STEAM MUCH \\
\hline E: & fight / steam & LAST-TIME BOX TRAINING IX STEAM MUCH \\
\hline G: & Dieb / Sieb & YESTERDAY KITCHEN IX OLD THIEF THREE SIEVE STEAL \\
\hline E: & thief / sieve & YESTERDAY KITCHEN IX OLD MAN THREE SIEVE STEAL \\
\hline G: & Fahne / Sahne & POSS $_{1}$ SOCCER FLAG IX CREAM SMACK $_{\mathrm{CL}}$ \\
\hline E: & flag / cream & POSS $_{1}$ SOCCER SHIRT IX CREAM SMACK $_{\mathrm{CL}}$ \\
\hline G: & Narbe / Farbe & T-I-M POSS 3 SCAR IX COLOR SLOWLY CHANGE \\
\hline E: & scar / color & T-I-M POSS 3 PANTS IX COLOR SLOWLY CHANGE \\
\hline G: & Tisch / Fisch & DINNER POSS 1 MOTHER TABLE IX FISH CUT ${ }_{C L}$ \\
\hline E: & table / fish & DINNER POSS 1 MOTHER KITCHEN IX FISH CUT $\mathrm{CL}_{\mathrm{L}}$ \\
\hline G: & Gabel / Kabel & POLICE INFO $_{\mathrm{PL}}$, PLEASE METAL FORK IX WIRE CONTACT MUST-NEG \\
\hline E: & fork / wire & POLICE INFO $_{\mathrm{PL}}$, PLEASE METAL KNIFE IX WIRE CONTACT MUST-NEG \\
\hline G: & Bericht / Gericht & POSS $_{1}$ INSURANCE ACCIDENT REPORT FOR COURT REFUSE \\
\hline E: & report / court & POSS $_{1}$ INSURANCE ACCIDENT REQUEST FOR COURT REFUSE \\
\hline G: & Paar / Harr & BERLIN IX EXIST COUPLE BOTH HAIR RED \\
\hline E: & couple / hair & BERLIN IX EXIST SIBLINGS BOTH HAIR RED \\
\hline G: & Hahn / Zahn & USUALLY ROOSTER IX ${ }_{\mathrm{PL}}$ TOOTH EXIST-NEG \\
\hline E: & rooster / tooth & USUALLY BIRD IX $X_{\mathrm{PL}}$ TOOTH EXIST-NEG \\
\hline $\begin{array}{l}\text { G: } \\
\text { F. }\end{array}$ & Wand / Hand & KINDERGARTEN IX WALL MANY HAND PRINT ${ }_{\mathrm{CL}}$ \\
\hline E: & wall / hand & KINDERGARTEN IX PICTURE MANY HAND PRINT $\mathrm{CL}_{\mathrm{L}}$ \\
\hline G: & Land / Sand & ÄGYPTEN IX COUNTRY MUCH SAND EXIST \\
\hline E: & country / sand & ÄGYPTEN IX AREA MUCH SAND EXIST \\
\hline G: & Mantel / Hantel & SPORT FINISH, MAN POSS 3 COAT IX BARBELL POCKET ${ }_{\mathrm{CL}}$ \\
\hline E: & coat / barbell & SPORT FINISH, MAN POSS $_{3}$ BAG IX BARBELL POCKET $T_{\mathrm{CL}}$ \\
\hline
\end{tabular}


G: Hase / Nase

E: rabbit / nose

G: Haus / Maus

E: house / mouse

G: Hose / Rose

E: pants / rose

G: Nummer / Kummer

E: number / grief

G: Hund / Mund

E: dog / mouth

G: Mut / Hut

E: courage / hat

G: Wette / Kette

E: bet / chain

G: Kind / Wind

E: child / wind

G: Koch / Loch

E: chef / hole

G: Topf / Kopf

E: pot / head

G: Liege / Ziege

E: lounger / goat

G: Lupe / Hupe

E: magnifier / horn

G: Macht / Nacht

E: power / night

G: Nonne / Sonne

E: nun / sun

G: Rost / Post

E: rust / post

G: Regel / Segel

E: rule / sail

G: Riese / Wiese

E: giant / grassland

G: Kasse / Tasse

E: cashier / cup

G: Turm / Wurm

E: tower / worm

G: Welt / Zelt

E: world / tent

G: Wolf / Golf

E: wolf / golf
POSS $_{1}$ DAUGTHER POSS 3 a RABBIT IX NOSE SCRATCH CL $_{\text {L }}$ POSS $_{1}$ DAUGTHER POSS $_{3 \mathrm{a}}$ CAT IX NOSE SCRATCH $\mathrm{CL}_{\mathrm{CL}}$

LAST WEEK POSS ${ }_{1}$ HOUSE IX MOUSE HIDE

LAST WEEK POSS 1 KITCHEN IX MOUSE HIDE

WEDDINGDAY POSS ${ }_{1}$ PARTNER POSS $_{3 \mathrm{a}}$ PANTS IX $_{1}$ ROSE PUT-IN

WEDDINGDAY POSS ${ }_{1}$ PARTNER POSS $_{3 \mathrm{a}}$ SHIRT IX 1 ROSE PUT-IN

POSS $_{1}$ PARTNER IX 3 a SICK. IX 1 NUMBER FOR GRIEF CALL

POSS $_{1}$ PARTNER IX 3 a SICK. IX 1 DOCTOR FOR GRIEF CALL

POSS $_{1}$ GRANDPA POSS 3 DOG IX MOUTH STINK

POSS $_{1}$ GRANDPA POSS $_{3}$ CAT IX MOUTH STINK

YESTERDAY IX ${ }_{1}$ VERY COURAGE GO HAT STRANGE BUY

YESTERDAY IX ${ }_{1}$ VERY FEAR GO HAT STRANGE BUY

YESTERDAY WE-DUAL STRANGE BET TOPIC CHIAN IX STABIL OR BREAK

YESTERDAY WE-DUAL STRANGE TALK TOPIC CHIAN IX STABIL OR BREAK

AUTUMN POSS 1 CHILD IX WIND LOVE

AUTUMN POSS 1 BROTHER IX WIND LOVE

RESTAURANT CHEF IX HOLE STUMBLE-IN ${ }_{C L}$

RESTAURANT MAN IX HOLE STUMBLE-IN ${ }_{\mathrm{CL}}$

CHILD IX POT POSS $3 \mathrm{a}$ HEAD PUT-ON $\mathrm{N}_{\mathrm{CL}}$

CHILD IX BOWL POSS $3 \mathrm{a}$ HEAD PUT-ON $\mathrm{N}_{\mathrm{CL}}$

EGYPT HOTEL IX LOUNGER IX GOAT STAND-ON

EGYPT HOTEL IX TABLE IX GOAT STAND-ON $\mathrm{N}_{\mathrm{CL}}$

PLAY CAR IX CHILD MAGNIFIER FOR HORN NEED

PLAY CAR IX CHILD BATTERY FOR HORN NEED

MANY KING POSS 3 POWER OFTEN NIGHT PLAN

MANY KING POSS 3 WAR OFTEN NIGHT PLAN

CLOISTER IX NUN LOVE SUN LOOK-AT

CLOISTER IX MONK LOVE SUN LOOK-AT

LETTERBOX MUCH RUST IX POST BAD FOR

LETTERBOX MUCH HOLE IX POST BAD FOR

POSS $_{1}$ GRANDPA MANY RULE FOR SAIL SETTING KNOW

POSS 1 GRANDPA MANY EXPERIENCE FOR SAIL SETTING HAVE

CHILDREN\#TALE GIANT IX GRASSLAND STAMPERS-ACCROSS ${ }_{\mathrm{CL}}$ CHILDREN\#TALE MAN IX GRASSLAND STAMPERS-ACCROSS ${ }_{\mathrm{CL}}$

KITCHEN STORE WOMAN CHECKOUT IX CUP PAY

KITCHEN STORE WOMAN COUNTER IX CUP PAY

FAIRYTALE WOODS TOWER IX WORM CRAWL-UP ${ }_{\mathrm{CL}}$ FAIRYTALE WOODS CASTLE IX WORM CRAWL-UP ${ }_{\mathrm{CL}}$

LAST YEAR POSS ${ }_{1}$ FRIEND WORLD WITH TENT TRAVEL

LAST YEAR POSS ${ }_{1}$ FRIEND EUROPE WITH TENT TRAVEL

ANIMAL STORY CONTENT WOLF IX GOLF PLAY WISH ANIMAL STORY CONTENT RABBIT IX GOLF PLAY WISH 


\section{Chapter 3: Complete list of stimulus sentences; Exp. A (agreement verbs)}

\begin{tabular}{|c|c|c|}
\hline Agr. verb & Match & Missmatch \\
\hline HELP & $\begin{array}{l}\text { PUPIL IX }_{3 a} \text { MATHEMATICS PROBLEM. TODAY HOMEWORK }{ }_{1} \mathrm{HELP}_{3 \mathrm{a}} \\
\text { MY GRANDMA IX } \text { Ha }_{3 \mathrm{OLD}} \text { THEREFORE SHOPPING }{ }_{1} \mathrm{HELP}_{3 \mathrm{a}} \\
\text { MY FRIEND IX }_{3 \mathrm{a}} \text { STRESSED. MAYBE WORK }{ }_{1} \mathrm{HELP}_{3 \mathrm{a}} \\
\text { CHILD IX }_{3 \mathrm{a}} \text { HELPLESS. SHOES TYING }_{1} \mathrm{HELP}_{3 \mathrm{a}}\end{array}$ & $\begin{array}{l}\cdots 1 \mathrm{HELP}_{3 b} \\
\cdots 1 \mathrm{HELP}_{3 \mathrm{~b}} \\
\cdots 1 \mathrm{HELP}_{3 \mathrm{~b}} \\
\cdots 1 \mathrm{HELP}_{3 \mathrm{~b}}\end{array}$ \\
\hline GIVE & 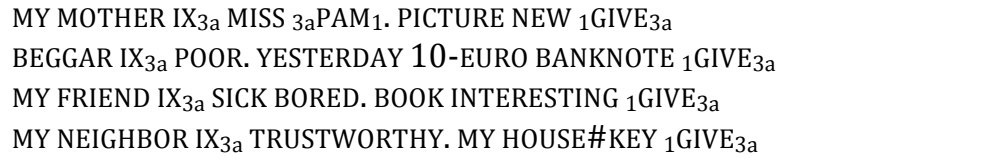 & $\begin{array}{l}\cdots 1 \mathrm{GIVE}_{3 \mathrm{~b}} \\
\cdots 1 \mathrm{GIVE}_{3 \mathrm{~b}} \\
\cdots 1 \mathrm{GIVE}_{3 \mathrm{~b}} \\
\cdots 1 \text { GIVE } \\
\cdots \mathrm{b}\end{array}$ \\
\hline VISIT & $\begin{array}{l}\text { MY GRANDMA IX }{ }_{3 a} \text { SICK. TODAY EVENING }{ }_{1} \text { VISIT }_{3 a} \\
\text { GERMAN\#DEAF\#CLUB PRESIDENT IX }{ }_{3 a} \text { DEAF. NEXT WEEK }{ }_{1} \text { VISIT }_{3 a} \\
\text { MY WORK\#COLLEAGUE IX }{ }_{3 a} \text { SICK. PATIENCE SOON }{ }_{1} \text { VISIT }_{3 a} \\
\text { MY FRIEND IX } 3 \text { a PREGNANT. TOMORROW NOON }{ }_{1} \text { VISIT }_{3 a}\end{array}$ & $\begin{array}{l}\cdots 1 \operatorname{VISIT}_{3 b} \\
\cdots 1 \operatorname{VISIT}_{3 b} \\
\cdots 1 \operatorname{VISIT}_{3 b} \\
\cdots 1 \operatorname{VISIT}_{3 b}\end{array}$ \\
\hline GIVE (as pres.) & $\begin{array}{l}\text { MY GRANDPA IX }{ }_{3 a} \text { BIRTHDAY. CIGAR EXPENSIVE }{ }_{1} \text { GIVE-PRESENT } 3 a \\
\text { MY PARTNER IX }_{3 a} \text { FAITHFUL. RING GOLD }{ }_{1} \text { GIVE-PRESENT } 3 \text { a } \\
\text { CHILD IX }_{3 a} \text { SAD. THEREFORE CHOCOLATE }{ }_{1} \text { GIVE-PRESENT }_{3 a} \\
\text { MY AUNT IX }_{3 a} \text { SILVER\#WEDDING. IKEA COUPON }{ }_{1} \text { GIVE-PRESENT }_{3 a}\end{array}$ & $\begin{array}{l}\cdots{ }_{1} \text { GIVE-PR } 3 b \\
\cdots{ }_{1} \text { GIVE-PR } \\
\cdots{ }_{1 b} \text { GIVE-PR } \\
\cdots{ }_{3 b} \text { GIVE-PR } \\
\cdots b\end{array}$ \\
\hline FAX & 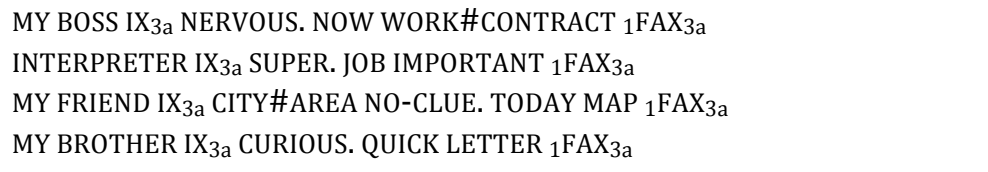 & 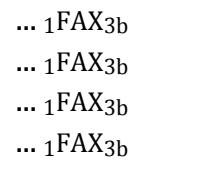 \\
\hline ASK & $\begin{array}{l}\text { MY SISTER IX }{ }_{3 a} \text { MATHEMATICS CLEVER. TOPIC PERCENT CALCULATION }{ }_{1} \text { ASK }_{3 a} \\
\text { MY GRANDMA IX } 3 \text { COOK SUPER. CAKE RECIPE }{ }_{1} \text { ASK }_{3 a} \\
\text { MY UNCEL IX } \\
\text { Wa POLICEMAN. DIRECTION DESCRIPTION }{ }_{1} \text { ASK }_{3 a} \\
\text { WOMAN IX } 3 \text { S SIGNLANGUAGE\#LECTURER. SIGN NEW }{ }_{1} \text { ASK }_{3 a}\end{array}$ & $\begin{array}{l}\ldots 1{ }_{1} \mathrm{ASK}_{3 \mathrm{~b}} \\
\ldots 1 \mathrm{ASK}_{3 \mathrm{~b}} \\
\ldots 1 \mathrm{ASK}_{3 \mathrm{~b}} \\
\ldots 1 \mathrm{ASK}_{3 \mathrm{~b}}\end{array}$ \\
\hline EXPLAIN & $\begin{array}{l}\text { CHILD IX }_{3 a} \text { MATHEMATICS PROBLEM. CONTENT STEP-BY-STEP }{ }_{1} \text { EXPLAIN }_{3 a} \\
\text { LAWYER IX }_{3 a} \text { NEUTRAL. SITUATION ACCIDENT }{ }_{1} \text { EXPLAIN }_{3 a} \\
\text { TRAINEE IX }_{3 a} \text { NO-CLUE. MACHINE DIFFICULT }{ }_{1} \text { EXPLAIN }_{3 a} \\
\text { MY COLLEAGUE DEAF\#FIELD INTERESTED. DEAF\#CULTURE }{ }_{1} \text { EXPLAIN }_{3 a}\end{array}$ & $\begin{array}{l}\cdots{ }_{1} \operatorname{EXPLAIN}_{3 b} \\
\cdots{ }_{1} \operatorname{EXPLAIN}_{3 b} \\
\cdots{ }_{1} \operatorname{EXPLAIN}_{3 b} \\
\ldots{ }_{1} \operatorname{EXPLAIN}_{3 b}\end{array}$ \\
\hline LEND & $\begin{array}{l}\text { MY PARTNER STUDYING. BOOK IMPORTANT }{ }_{1} \text { LEND }_{3 a} \\
\text { MY SISTER IX } 3 \text { a BROKE. } 100 \text { EURO }_{1} \text { LEND }_{3 a} \\
\text { MY FRIEND IX } 3 \text { COLD. JACKET WARM }{ }_{1} \text { LEND }_{3 a} \\
\text { MY SON IX } 3 \text { a GROWN-UP. MY CAR }{ }_{1} \text { LEND }_{3 a}\end{array}$ & $\begin{array}{l}\ldots 1 \operatorname{LEND}_{3 b} \\
\cdots 1 \operatorname{LEND}_{3 b} \\
\cdots 1 \operatorname{LEND}_{3 \mathrm{~b}} \\
\cdots{ }_{1} \operatorname{LEND}_{3 \mathrm{~b}}\end{array}$ \\
\hline SEND & $\begin{array}{l}\text { MY DAUGHTER IX } 3 \text { a TOMORROW BIRTHDAY. LETTER LONG }{ }_{1} \text { SEND }_{3 a} \\
\text { WOMAN IX } 3 \text { a NICE. WISH SMS }{ }_{1} \text { SEND }_{3 a} \text {. } \\
\text { PROFESSOR IX }_{3 a} \text { GOOD-NATURED. HOMEWORK GLADLY }{ }_{1} \text { SEND }_{3 a} \\
\text { ARTIST IX }_{3 a} \text { SUPER. MY DRAWING PICTURE }{ }_{1} \text { SEND }_{3 a}\end{array}$ & $\begin{array}{l}\ldots 1 \operatorname{SEND}_{3 b} \\
\ldots 1 \operatorname{SEND}_{3 b} \\
\ldots 1 \operatorname{SEND}_{3 b} \\
\ldots 1 \operatorname{SEND}_{3 \mathrm{~b}}\end{array}$ \\
\hline INFORM & $\begin{array}{l}\text { MY FATHER IX }{ }_{3 a} \text { SOCCER FAN. NEXT MATCH DATE }{ }_{1} \text { INFORM }_{3 a} \\
\text { MY BOSS IX }_{3 a} \text { STRESSED. THEREFORE DAILY\# ROUTINE }_{1} \text { INFORM }_{3 a} \\
\text { MY MOTHER IX }_{3 a} \text { SURPRISED. TODAY MOVE }{ }_{1} \text { INFORM }_{3 a} \\
\text { CHILD IX }_{3 a} \text { NAUGHTY. PUNISHMENT HOUSE\# }_{\text {ARREST }}{ }_{1} \text { INFORM }_{3 a}\end{array}$ & $\begin{array}{l}\cdots 1_{1} \text { INFORM }_{3 b} \\
\cdots 1 \text { INFORM }_{3 b} \\
\cdots 1 \text { INFORM }_{3 b} \\
\cdots \text { INFORM }_{3 b}\end{array}$ \\
\hline
\end{tabular}


Chapter 3: Complete list of stimulus sentences; Exp. B (plain verbs)

\begin{tabular}{|c|c|c|c|c|}
\hline Plain verb & Match & Mismatch & Body-anc. & Loc of art. \\
\hline \multirow[t]{4}{*}{ LIKE } & I TEDDY LIKE & ... LIKE3a & yes & chest \\
\hline & I FLOWER LIKE & ... LIKE 3 a & & \\
\hline & I SOFA LIKE & ... LIKE 3 a & & \\
\hline & I DOG LIKE & ... LIKE $_{3 a}$ & & \\
\hline \multirow[t]{4}{*}{ LOVE } & I MY PARTNER LOVE & ... LOVE 3 a & yes & chest \\
\hline & I GRANDPA LOVE & ... LOVE 3 a & & \\
\hline & I FATHER LOVE & ... LOVE 3 a & & \\
\hline & I CAT LOVE & ... LOVE 3 a & & \\
\hline \multirow[t]{4}{*}{ WAIT } & I FRIEND WAIT & ... WAIT $_{3 a}$ & yes & chest \\
\hline & I GRANDMA WAIT & $\ldots$ WAIT $_{3 a}$ & & \\
\hline & I GRANDPA WAIT & ... WAIT $_{3 a}$ & & \\
\hline & I FATHER WAIT & ... WAIT3a & & \\
\hline \multirow[t]{4}{*}{ PLAY } & I TENNIS PLAY & ... PLAY3a & no & neutral space \\
\hline & I CARDS PLAY & ... PLAY3a & & \\
\hline & I CHESS PLAY & $\ldots \mathrm{PLAY}_{3 \mathrm{a}}$ & & \\
\hline & I SOCCER PLAY & ... PLAY $3 \mathrm{a}$ & & \\
\hline \multirow[t]{4}{*}{ UNDERSTAND } & I AMERICA SIGN UNDERSTAND & ... UNDERSTAND 3 a & no & head \\
\hline & I PROBLEM UNDERSTAND & ... UNDERSTAND 3 a & & \\
\hline & I CONNECTION UNDERSTAND & ... UNDERSTAND 3 a & & \\
\hline & I MATHEMATICS UNDERSTAND & ... UNDERSTAND3a & & \\
\hline \multirow[t]{4}{*}{ BUY } & I BOOK BUY & ... BUY $3 \mathrm{a}$ & no & neutral space \\
\hline & I APPEL BUY & $\ldots \mathrm{BUY}_{3 \mathrm{a}}$ & & (non-dom hand) \\
\hline & I LAPTOP BUY & $\ldots \mathrm{BUY}_{3 \mathrm{a}}$ & & \\
\hline & I BREAD BUY & $\ldots \mathrm{BUY}_{3 \mathrm{a}}$ & & \\
\hline \multirow[t]{4}{*}{ KNOW } & I MAN IX $3 a$ KNOW & ... KNOW $_{3 a}$ & yes & head \\
\hline & I PROBLEM IX $3 \mathrm{a}$ KNOW & $\ldots \mathrm{KNOW}_{3 \mathrm{a}}$ & & \\
\hline & I DOCTOR IX 3 KNOW & $\ldots$ KNOW $_{3 a}$ & & \\
\hline & I SITUATION IX 3 Ka KNOW & .... $\mathrm{KNOW}_{3 \mathrm{a}}$ & & \\
\hline \multirow[t]{4}{*}{ GRASP } & I HOMEWORK GRASP & ... GRASP 3 a & no & head \\
\hline & I TOPIC GRASP & $\ldots$ GRASP $_{3 a}$ & & \\
\hline & I PROBLEM GRASP & $\ldots$ GRASP $_{3 a}$ & & \\
\hline & I SIGN GRASP & ... GRASP $3 \mathrm{a}$ & & \\
\hline \multirow[t]{4}{*}{ FORGET } & I TOPIC FORGET & ... FORGET $3 \mathrm{a}$ & no & head \\
\hline & I SIGN FORGET & ... FORGET $3 \mathrm{a}$ & & \\
\hline & I EGG FORGET & ... FORGET $3 \mathrm{a}$ & & \\
\hline & I APPOINTMENT FORGET & ... FORGET $3 \mathrm{a}$ & & \\
\hline \multirow[t]{4}{*}{ TEST } & I BOY TEST & $\ldots \mathrm{TEST}_{3 \mathrm{a}}$ & no & neutral space \\
\hline & I HOMEWORK TEST & $\ldots$ TEST $_{3 a}$ & & (non-dom hand) \\
\hline & I GIRL TEST & ... TEST3a & & \\
\hline & I CAR TEST & ... TEST $3 \mathrm{a}$ & & \\
\hline \multirow[t]{4}{*}{ PLAN } & I VACATION PLAN & ... PLAN $_{3 a}$ & no & neutral space \\
\hline & I PRESENTATION PLAN & $\ldots$ PLAN $_{3 a}$ & & (non-dom hand) \\
\hline & I RELOCATION PLAN & $\ldots$ PLAN $_{3 a}$ & & \\
\hline & I PARTY PLAN & ... PLAN3a & & \\
\hline
\end{tabular}


Appendix B - Supplementary ERP figures and material

Chapter 1:

ERPS of non-action verbs - trigger handshape change
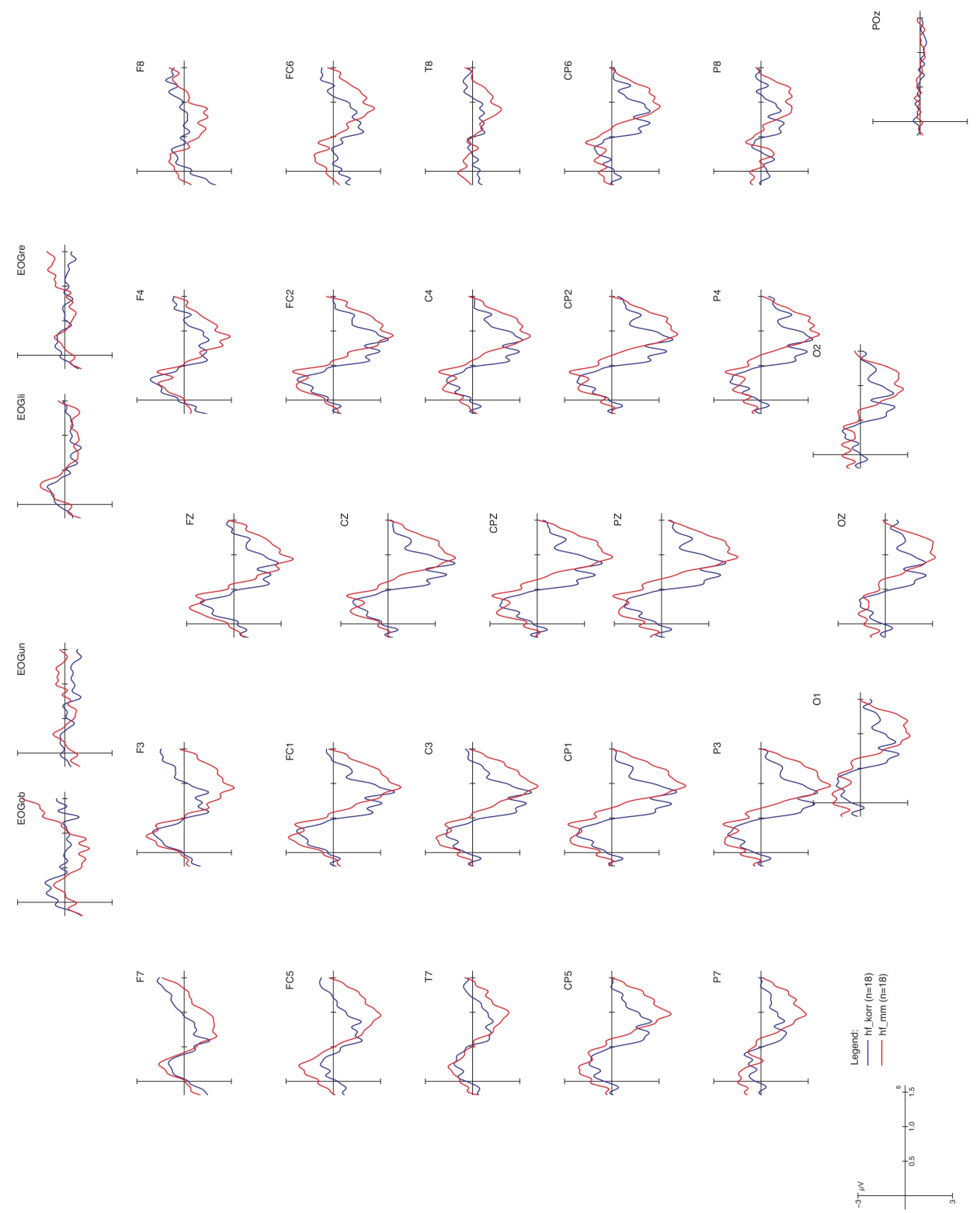
Chapter 1:

ERPs of non-action verbs - trigger target handshape
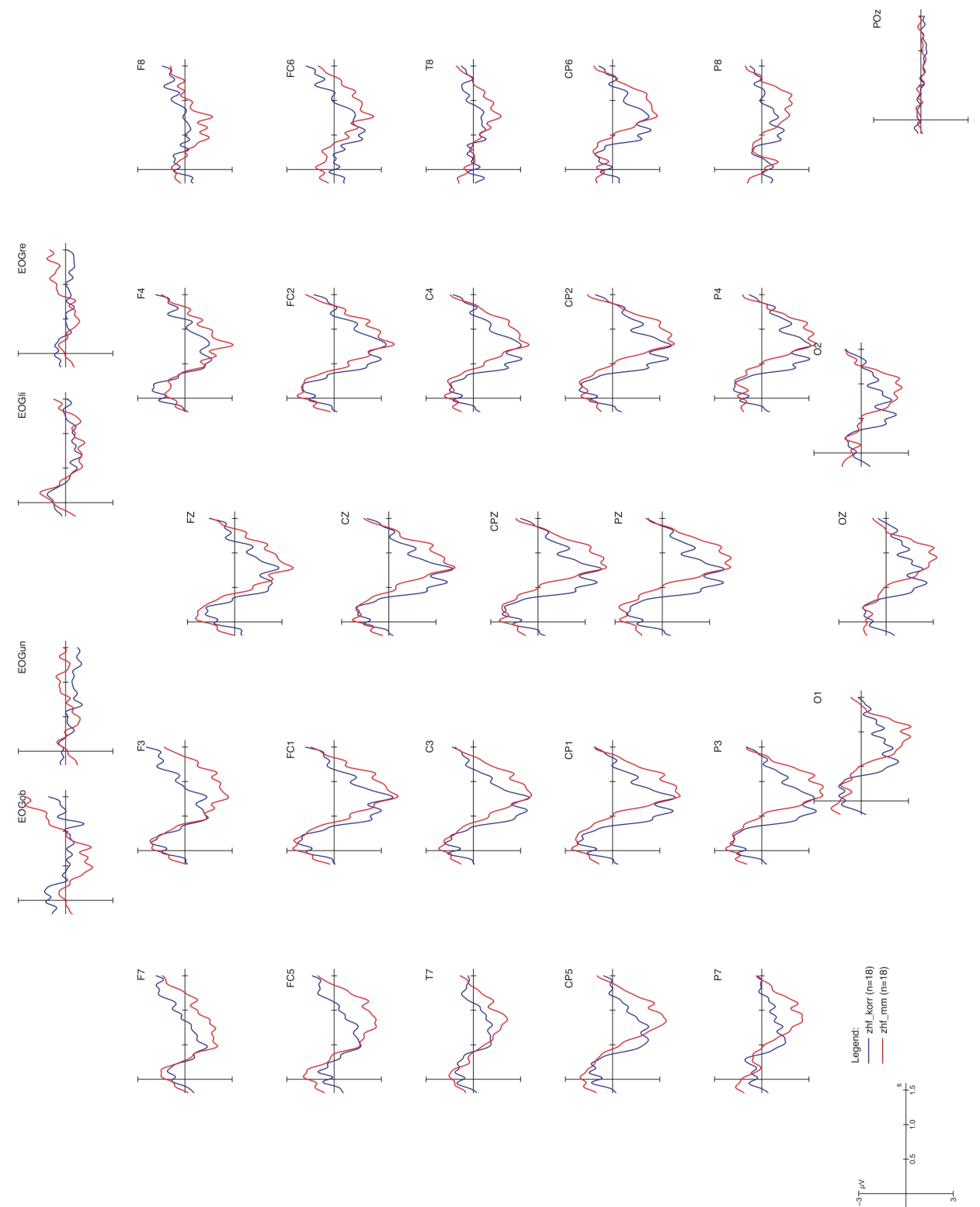


\section{Chapter 1:}

ERPs of non-action verbs - trigger sign onset
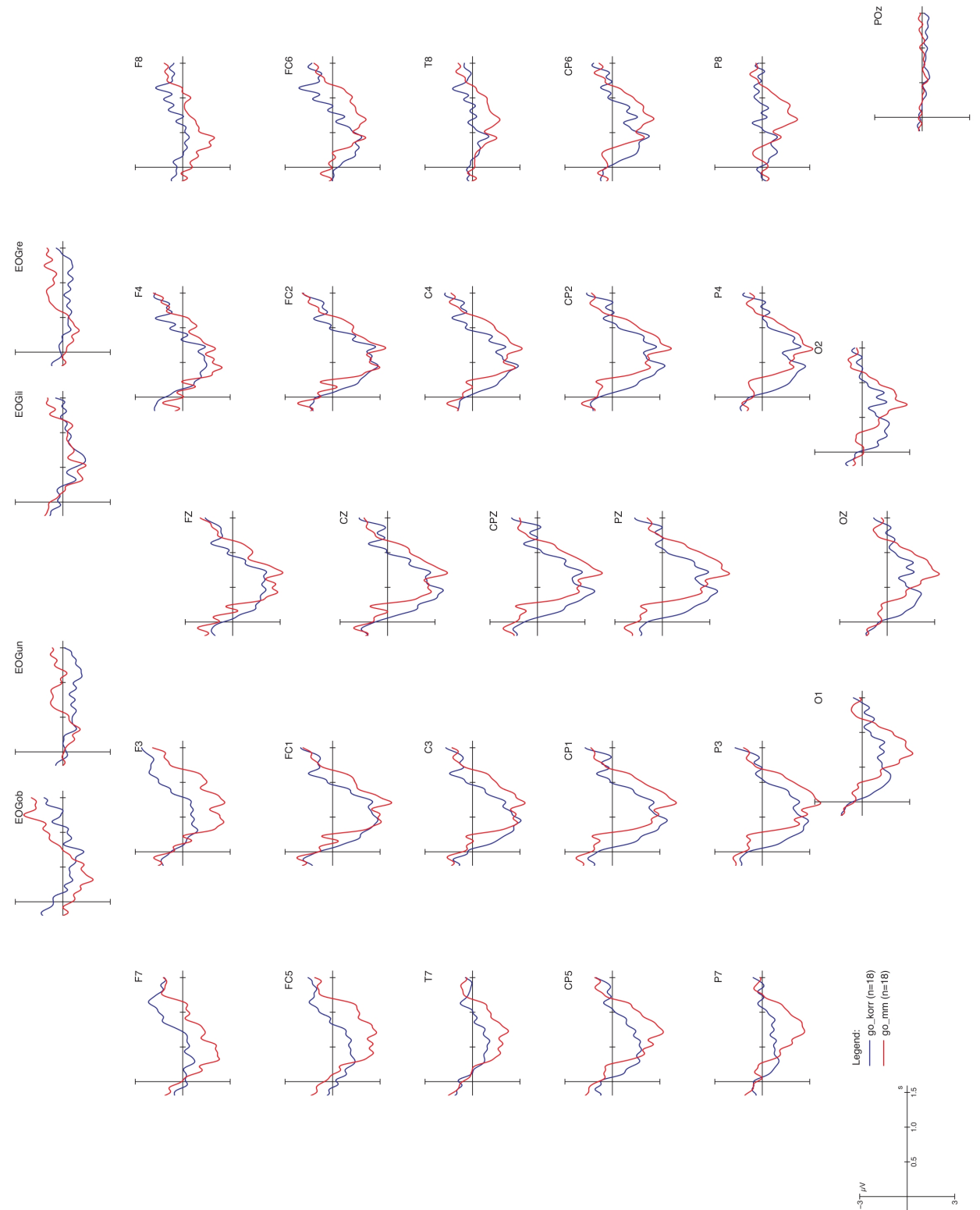


\section{Chapter 1:}

ERPs of action verbs - trigger handshape change
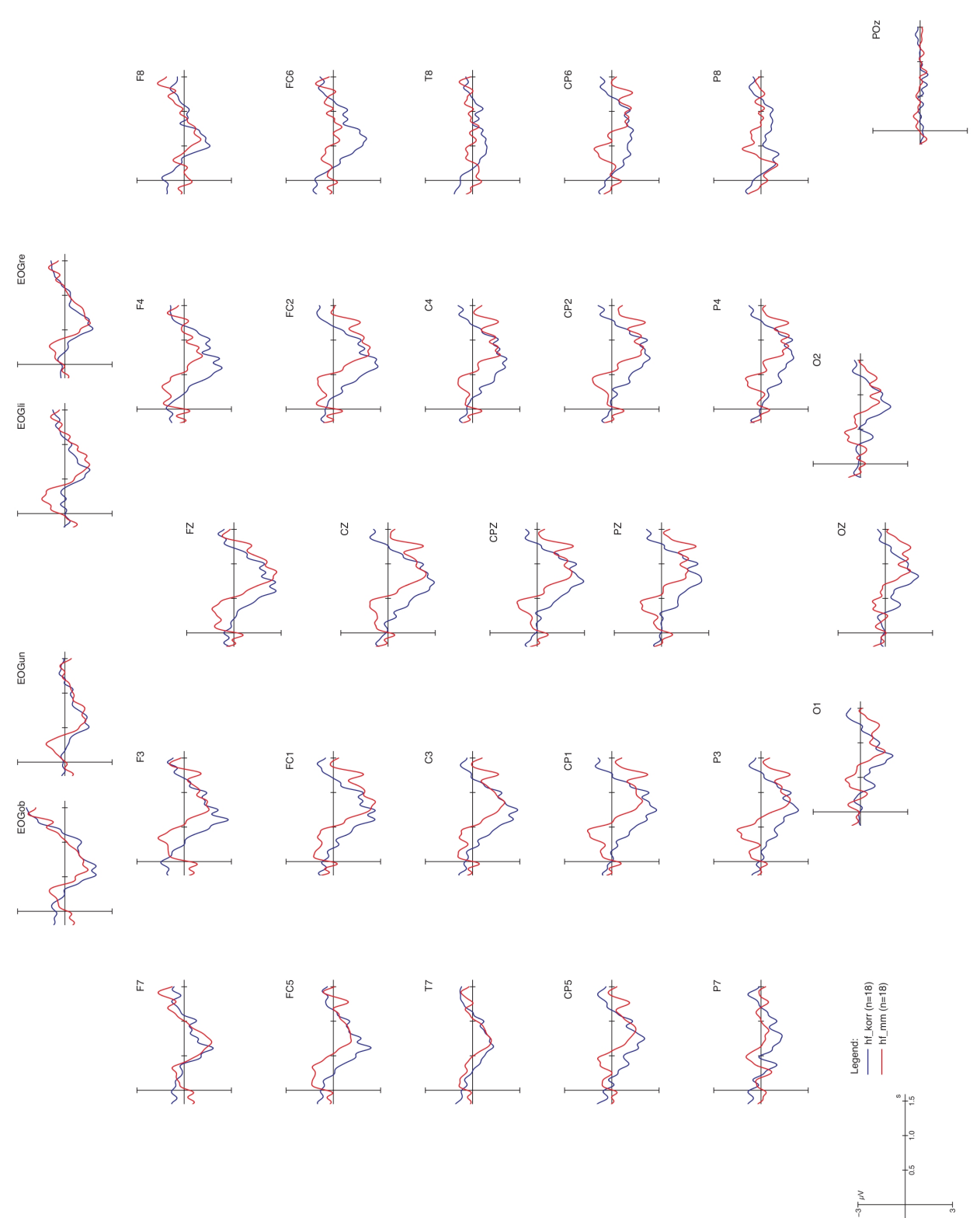


\section{Chapter 1:}

ERPs of action verbs - trigger target handshape
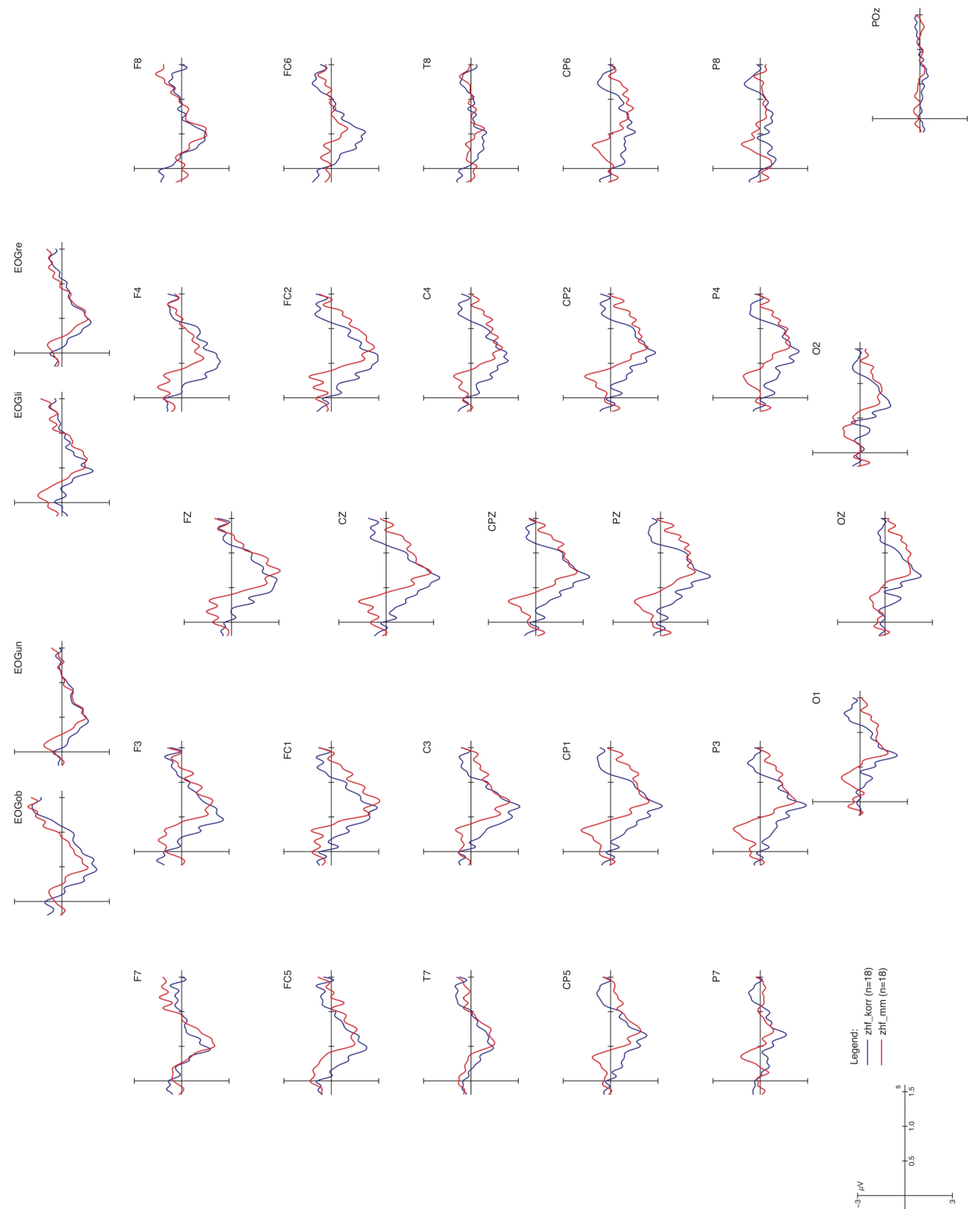


\section{Chapter 1:}

ERPs of action verbs - trigger sign onset
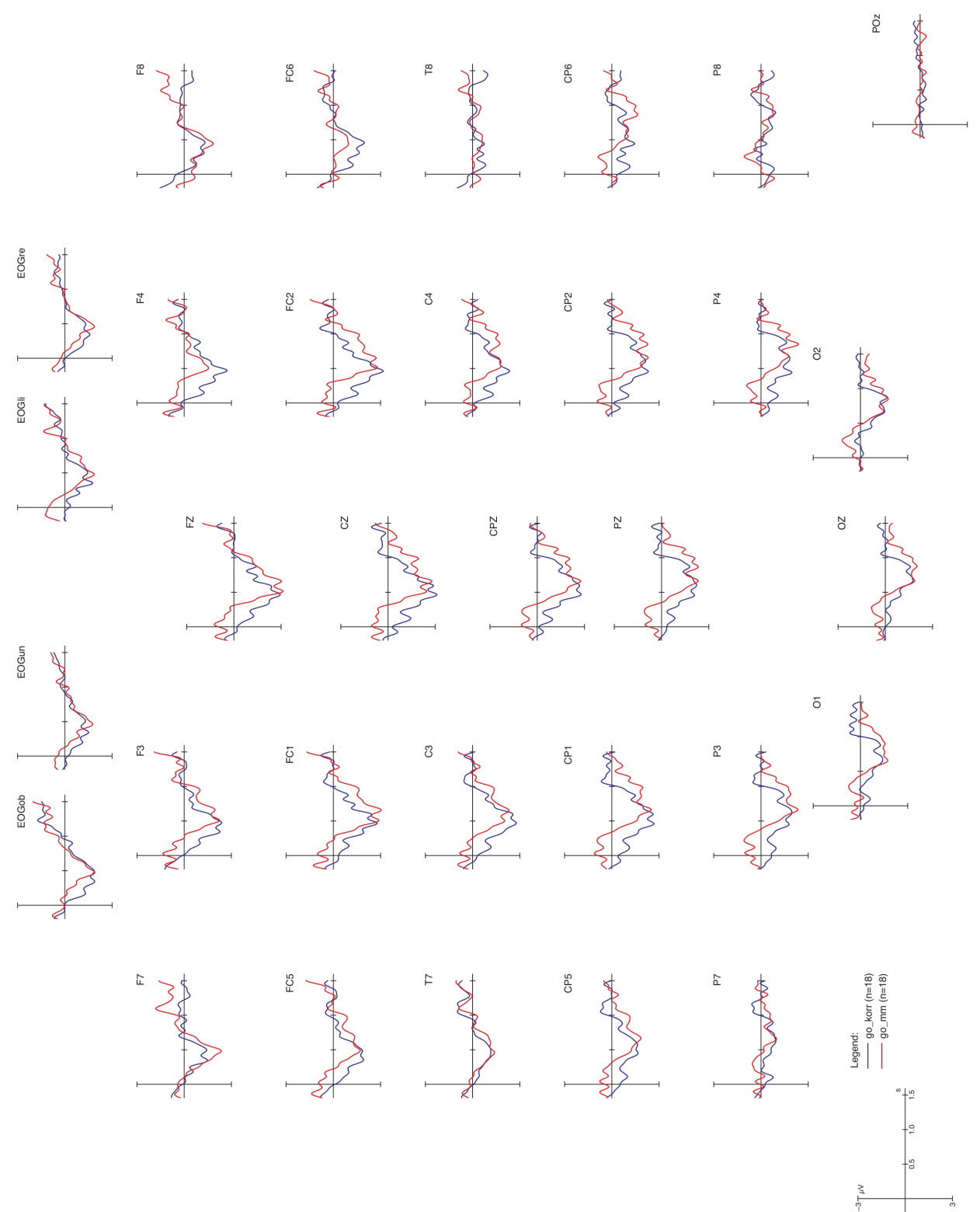
Appendix B

Chapter 2:

ERPS of DGS priming - trigger at target sign onset

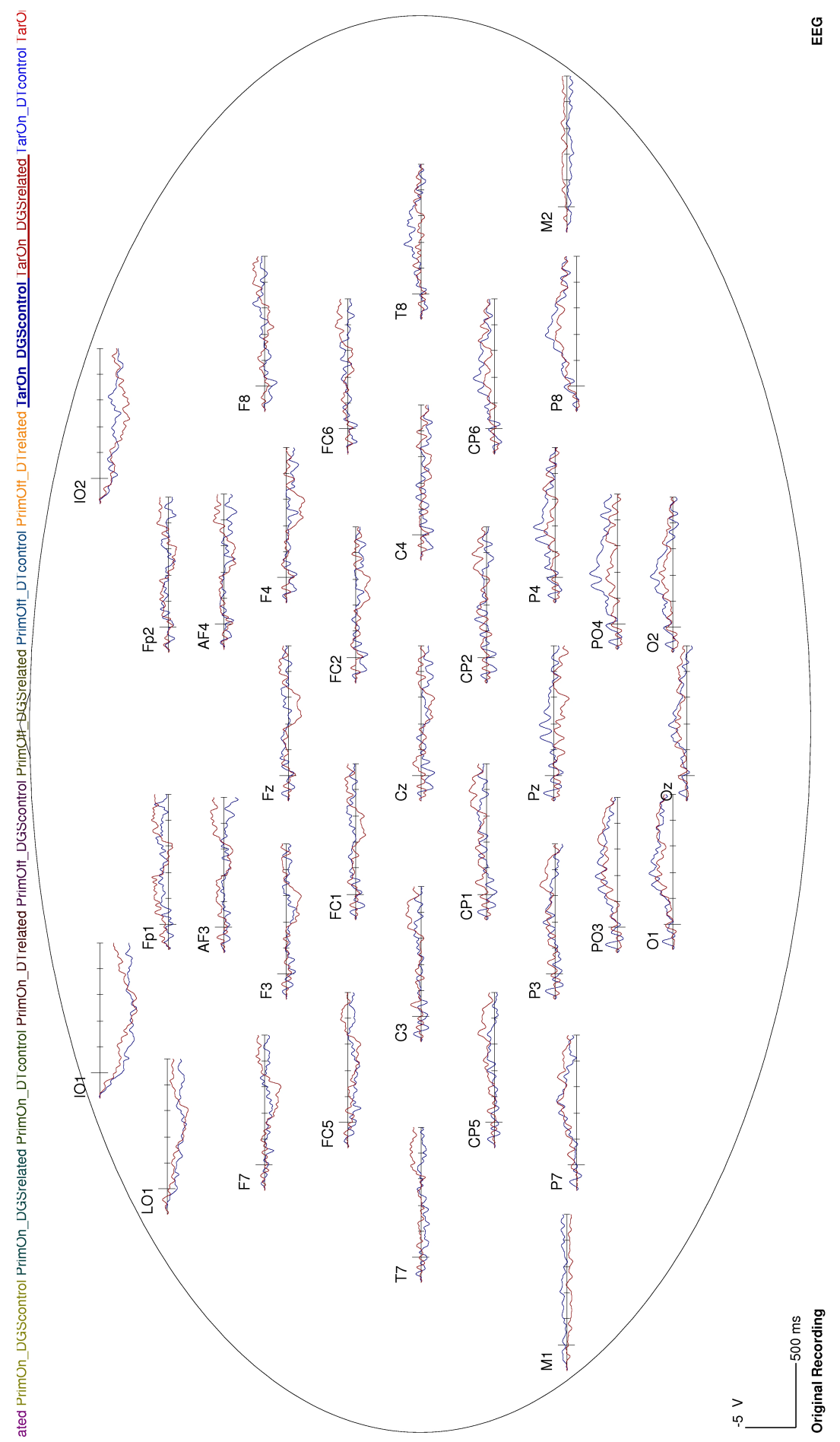


Appendix B

Chapter 2:

ERPS of German priming - trigger at target sign onset

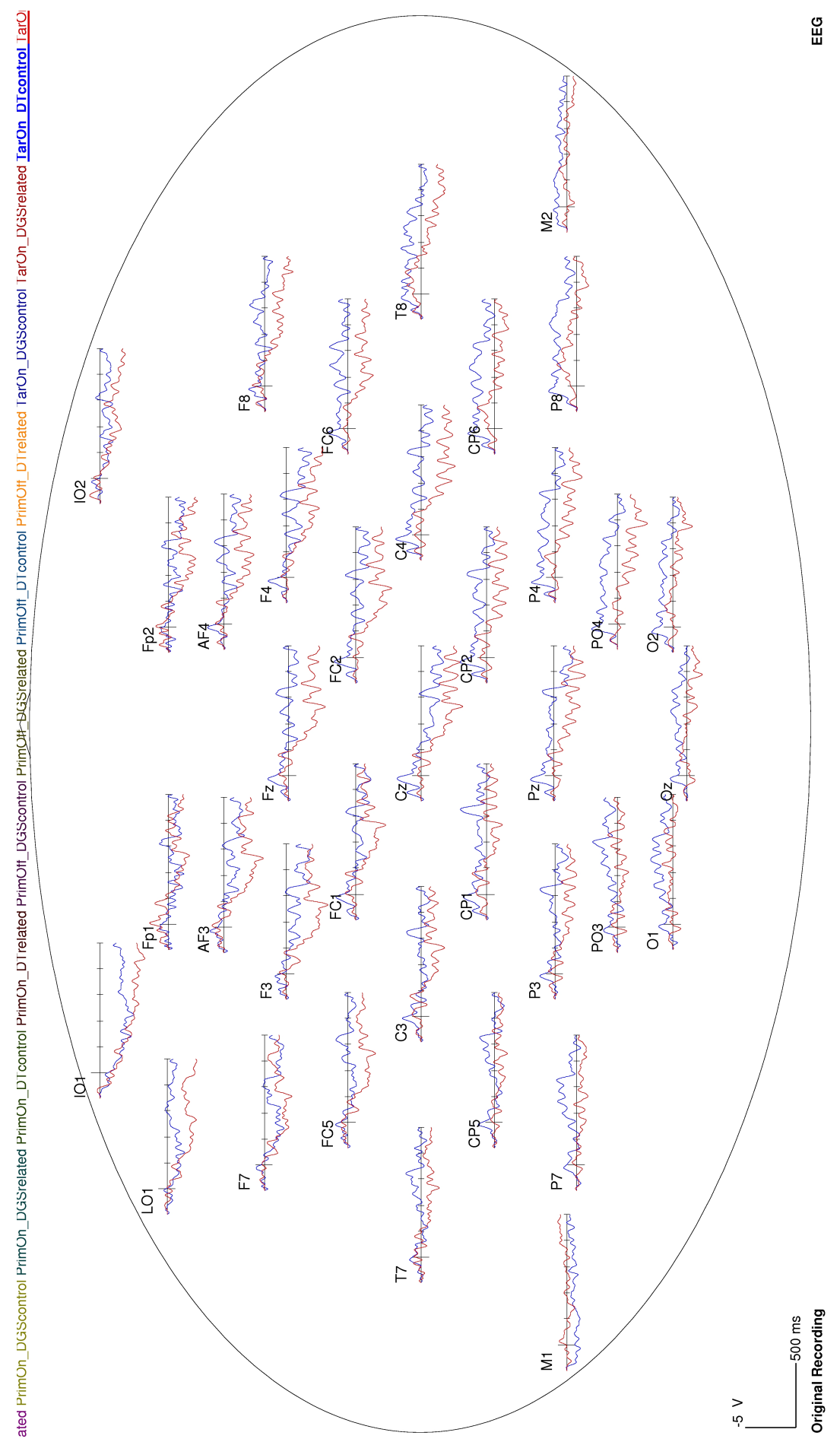




\section{Chapter 2:}

\section{Item rejection of translation task (German priming)}

1 = correct translated prime target pairs; 0 = incorrect trabslated prime target pairs: - = no translation

\begin{tabular}{|c|c|c|c|c|c|c|c|c|c|c|c|c|c|c|c|c|c|}
\hline prime & target & Vp1 & Vp2 & Vp3 & Vp4 & Vp5 & Vp6 & Vp7 & Vp8 & Vp9 & Vp10 & Vp11 & Vp12 & Vp13 & Vp14 & Vp15 & \\
\hline Engel & Angel & 1 & 1 & 1 & 1 & 1 & 1 & 1 & 0 & 1 & 1 & 1 & 1 & 1 & 1 & 1 & 14 \\
\hline Raum & Baum & 0 & 1 & 1 & - & 1 & 1 & 1 & 0 & 1 & 0 & 1 & 1 & 1 & 1 & 0 & 10 \\
\hline Wein & Bein & 0 & 1 & 0 & - & 0 & 0 & 0 & - & 0 & 0 & 0 & 0 & 0 & 0 & 0 & 1 \\
\hline Tier & Bier & 0 & 1 & - & 1 & 1 & 1 & 1 & 1 & 0 & 1 & 0 & 1 & 1 & 1 & 1 & 11 \\
\hline Tuch & Buch & 1 & 0 & 1 & 1 & 1 & 1 & 0 & 0 & 1 & 1 & 0 & 1 & 1 & 0 & 0 & 9 \\
\hline Mutter & Butter & 1 & 1 & 1 & 1 & 1 & 1 & 1 & 1 & 1 & 1 & 1 & 1 & 1 & 1 & 1 & 15 \\
\hline Dach & Schach & 0 & 1 & - & 1 & 0 & 0 & 1 & 0 & 1 & 1 & 0 & 1 & 1 & 1 & 1 & 9 \\
\hline Kampf & Dampf & 0 & 0 & 0 & 0 & 1 & 0 & 0 & 0 & 1 & 0 & 0 & 0 & 0 & 1 & 0 & 3 \\
\hline Dieb & Sieb & 0 & - & 0 & 0 & 0 & 0 & 1 & 0 & 0 & 0 & 0 & 0 & 0 & 1 & 0 & 2 \\
\hline Fahne & Sahne & 1 & 0 & 1 & 0 & 0 & 1 & 1 & 0 & 1 & 1 & 1 & 0 & 1 & 0 & 0 & 8 \\
\hline Narbe & Farbe & 0 & 0 & 1 & 1 & 1 & 1 & 1 & 0 & 0 & 1 & 0 & 0 & 0 & 1 & 0 & 7 \\
\hline Tisch & Fisch & 0 & 0 & 1 & 1 & 1 & 1 & 0 & 1 & 0 & 0 & 0 & 0 & 1 & 0 & 0 & 6 \\
\hline Gabel & Kabel & 0 & 0 & 0 & 0 & 0 & 0 & 1 & 0 & 0 & 0 & 0 & 0 & 0 & 0 & 0 & 1 \\
\hline Bericht & Gericht & 0 & 1 & 1 & 0 & 0 & 1 & 1 & 0 & 0 & 0 & 1 & 0 & 1 & 1 & 1 & 8 \\
\hline Paar & Haar & 1 & 1 & 1 & 1 & 1 & 1 & 1 & 1 & 1 & 1 & 1 & 1 & 1 & 1 & 1 & 15 \\
\hline Hahn & Zahn & 1 & 1 & 1 & 1 & 1 & 1 & 1 & 1 & 1 & 1 & 1 & 1 & 1 & 1 & 1 & 15 \\
\hline Wand & Hand & 1 & 0 & 1 & 1 & 1 & 1 & 1 & 1 & 1 & 1 & 1 & 1 & 1 & - & 1 & 13 \\
\hline Land & Sand & 0 & 0 & 0 & 1 & 1 & 1 & 0 & 0 & 0 & 0 & 0 & 0 & 0 & 0 & 0 & 3 \\
\hline Mantel & Hantel & 0 & 0 & 0 & 0 & 0 & 0 & 0 & 0 & 0 & 0 & 0 & 0 & 0 & 0 & 1 & 1 \\
\hline Hase & Nase & 1 & 0 & 0 & 1 & 1 & 1 & 1 & 1 & 1 & 1 & 1 & 1 & 1 & 1 & 1 & 13 \\
\hline Haus & Maus & 0 & 1 & 0 & 1 & 0 & 1 & 1 & 1 & 1 & 1 & - & 1 & 0 & 0 & 0 & 8 \\
\hline Hose & Rose & 0 & 0 & 1 & 1 & 1 & 1 & 0 & 0 & 0 & 1 & 0 & 1 & 1 & 1 & - & 8 \\
\hline Nummer & Kummer & 0 & 0 & 0 & 0 & 0 & 0 & 0 & 0 & 0 & 0 & 0 & 0 & 0 & 0 & 0 & $\mathbf{0}$ \\
\hline Hund & Mund & 1 & 1 & 1 & 1 & 1 & 1 & 1 & 0 & 1 & 1 & 1 & 1 & 1 & 1 & 1 & 14 \\
\hline Mut & Hut & 1 & 1 & 1 & 1 & 1 & 1 & 1 & 1 & 1 & 1 & 1 & 1 & 1 & 1 & 1 & 15 \\
\hline Wette & Kette & 0 & 0 & 1 & 0 & 0 & 0 & 1 & 0 & 0 & 0 & 0 & 0 & - & 0 & 1 & 3 \\
\hline Kind & Wind & 0 & 1 & 1 & 1 & 1 & 1 & 1 & 1 & 0 & 1 & 1 & 1 & 1 & 0 & 1 & 12 \\
\hline Koch & Loch & 1 & 1 & 1 & 0 & 0 & 1 & 1 & 0 & 0 & 0 & 1 & 1 & 1 & 0 & 1 & 9 \\
\hline Topf & Kopf & 1 & 0 & 1 & 1 & 1 & 0 & 1 & 1 & 0 & 1 & 1 & 1 & 1 & 1 & 0 & 11 \\
\hline Liege & Ziege & 0 & 0 & 0 & 0 & 0 & 0 & 0 & 0 & 0 & 0 & 0 & 1 & 0 & 1 & 0 & 2 \\
\hline Lupe & Hupe & 0 & 0 & 0 & 0 & 0 & 0 & 0 & 0 & 0 & 0 & 0 & 0 & 0 & 0 & 0 & 0 \\
\hline Macht & Nacht & 1 & 0 & 0 & 1 & 0 & 1 & 0 & 0 & 1 & - & 0 & 0 & 0 & 0 & 0 & 4 \\
\hline Nonne & Sonne & 1 & 0 & 1 & 1 & 1 & 1 & 1 & 1 & 1 & 1 & 0 & 1 & 1 & 1 & 1 & 13 \\
\hline Rost & Post & 0 & 1 & 0 & 1 & 0 & 0 & 1 & 1 & 0 & 0 & 0 & 1 & 0 & 1 & 0 & 6 \\
\hline Regel & Segel & 0 & 1 & 0 & 1 & 1 & 0 & 1 & 0 & 1 & 1 & 1 & 1 & 0 & 1 & 1 & 10 \\
\hline Riese & Wiese & 0 & 0 & 1 & 1 & 1 & 1 & 1 & 0 & 1 & 1 & 0 & 1 & 1 & 1 & 1 & 11 \\
\hline Kasse & Tasse & 0 & 0 & 0 & 0 & 0 & 0 & 0 & 0 & 0 & 0 & 0 & 0 & 0 & 0 & 0 & 0 \\
\hline Turm & Wurm & 0 & 0 & 0 & 0 & 0 & 0 & 0 & 0 & 0 & 0 & 1 & 1 & 0 & 0 & 0 & 2 \\
\hline Welt & Zelt & 0 & 0 & 1 & 1 & 1 & 1 & 1 & 0 & 1 & 1 & 1 & 1 & 1 & 0 & 1 & 11 \\
\hline Wolf & Golf & 0 & 1 & 0 & 0 & 0 & 0 & 1 & 0 & 1 & 1 & 1 & 1 & 1 & 0 & 0 & 7 \\
\hline
\end{tabular}




\section{Chapter 3:}

ERPs of agreement violation with agreement verbs - trigger handshape change
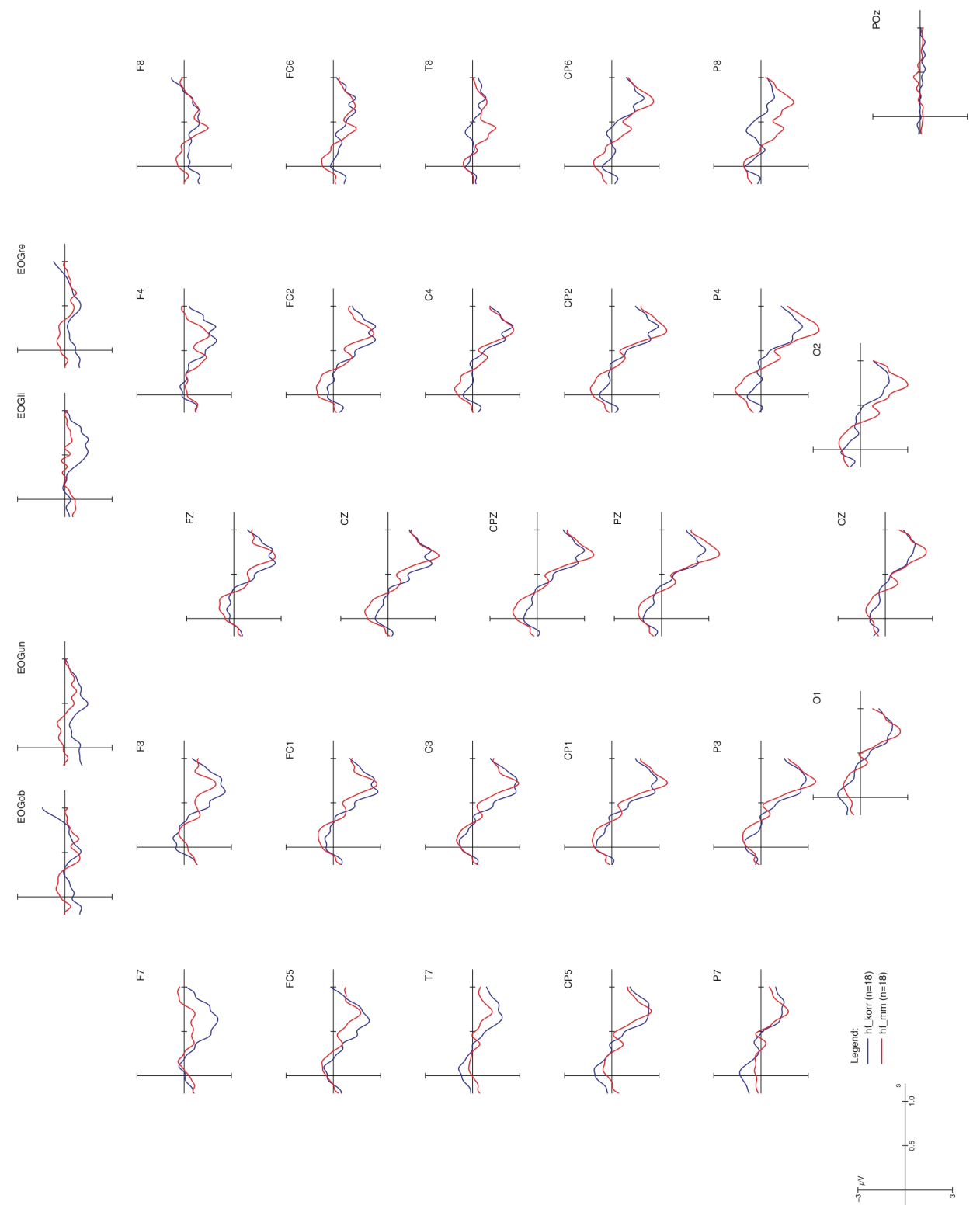


\section{Chapter 3:}

ERPs of agreement violation with agreement verbs - trigger target handshape
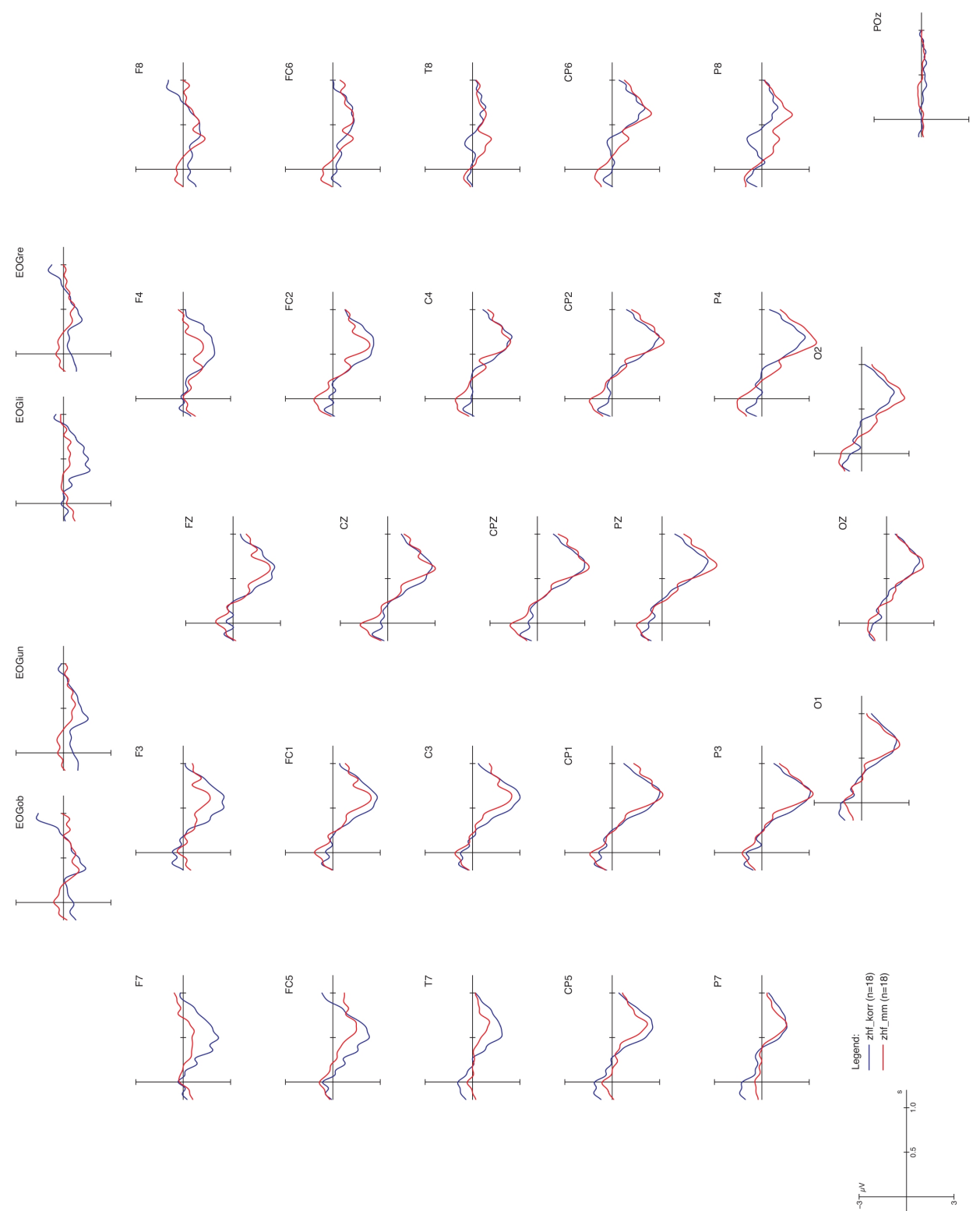


\section{Chapter 3:}

ERPs of agreement violation with agreement verbs - trigger nonmanual cues
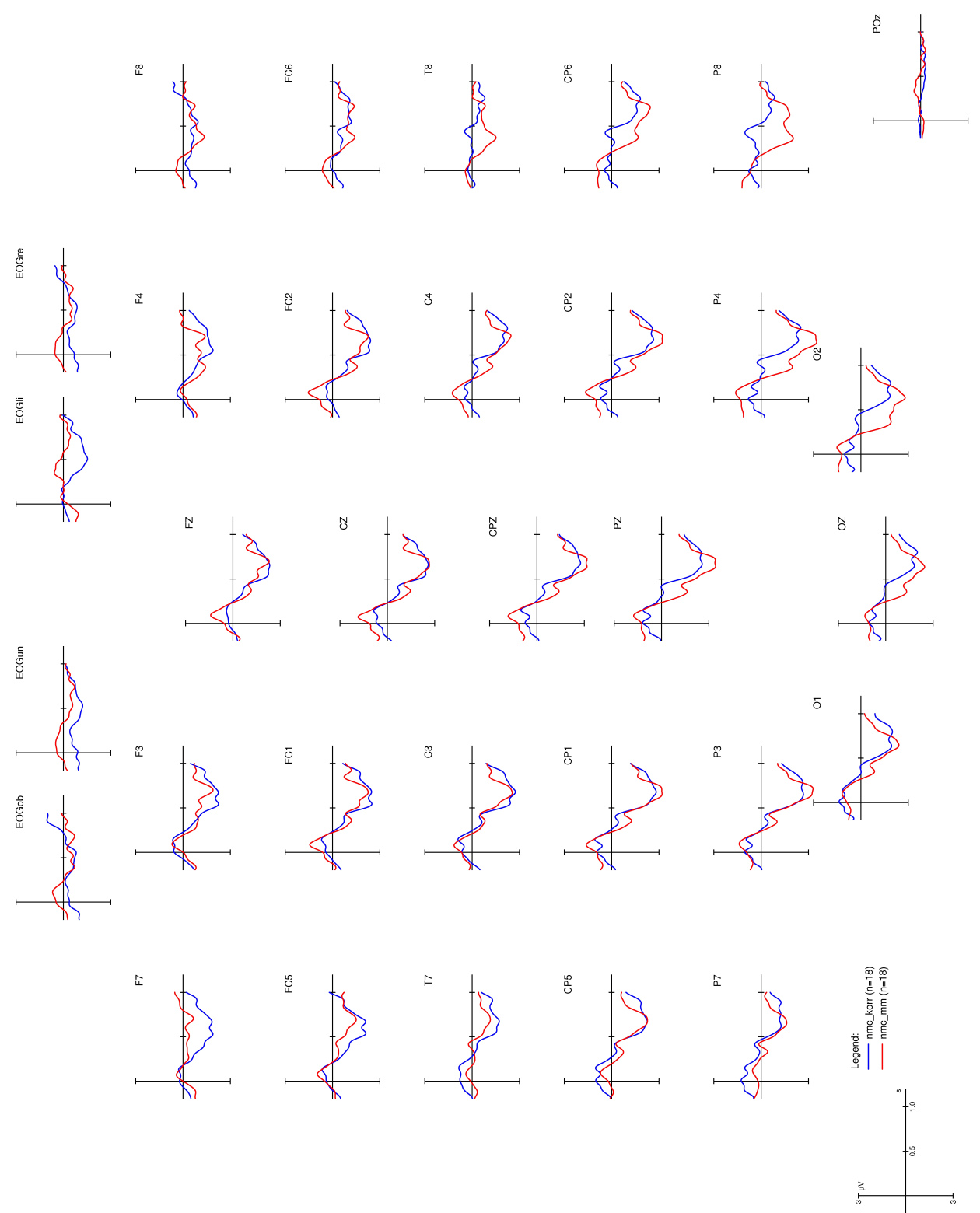


\section{Chapter 3:}

ERPs of agreement violation with agreement verbs - trigger sign onset
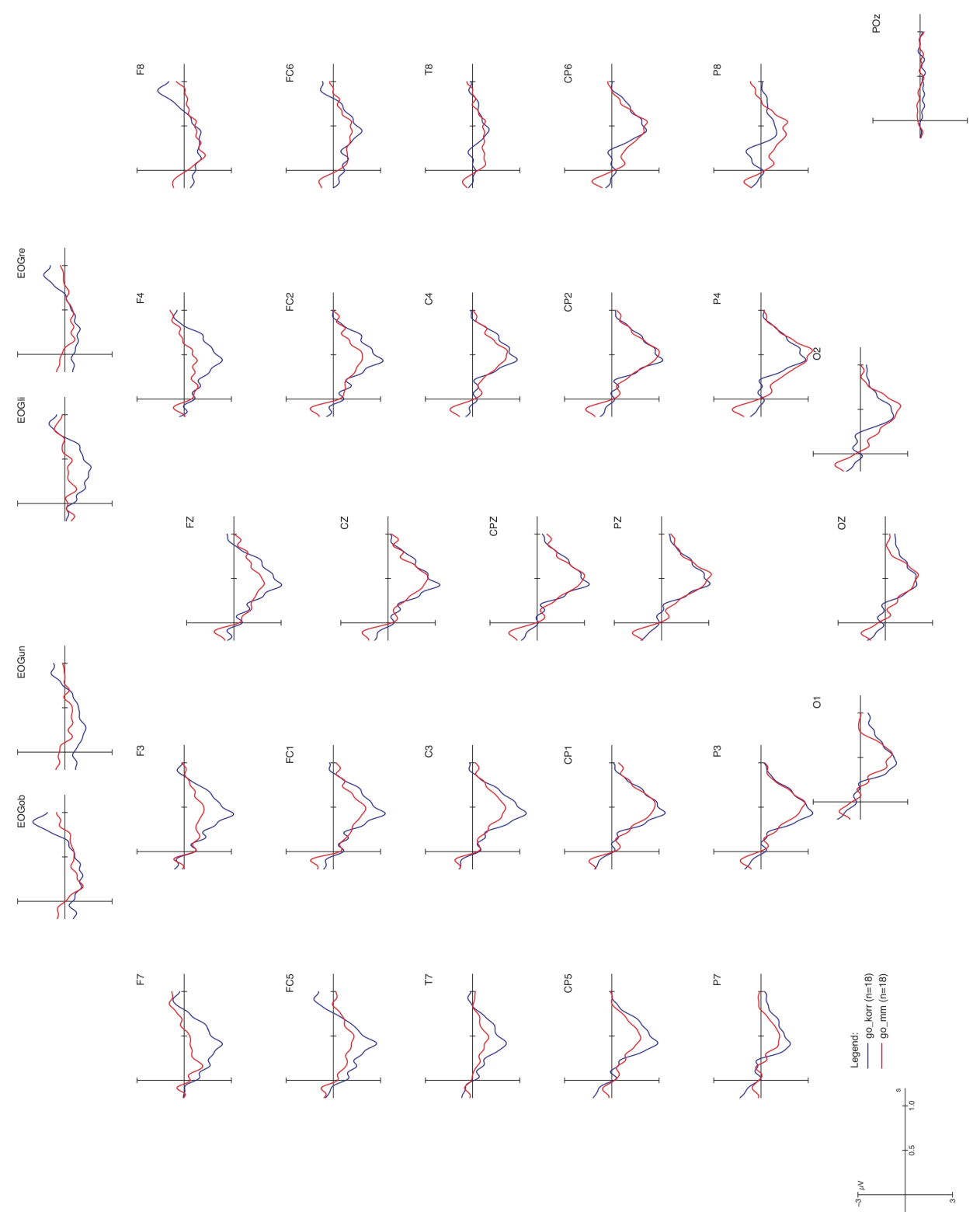
Chapter 3:

ERPs of agreement violation with plain verbs - trigger handshape change
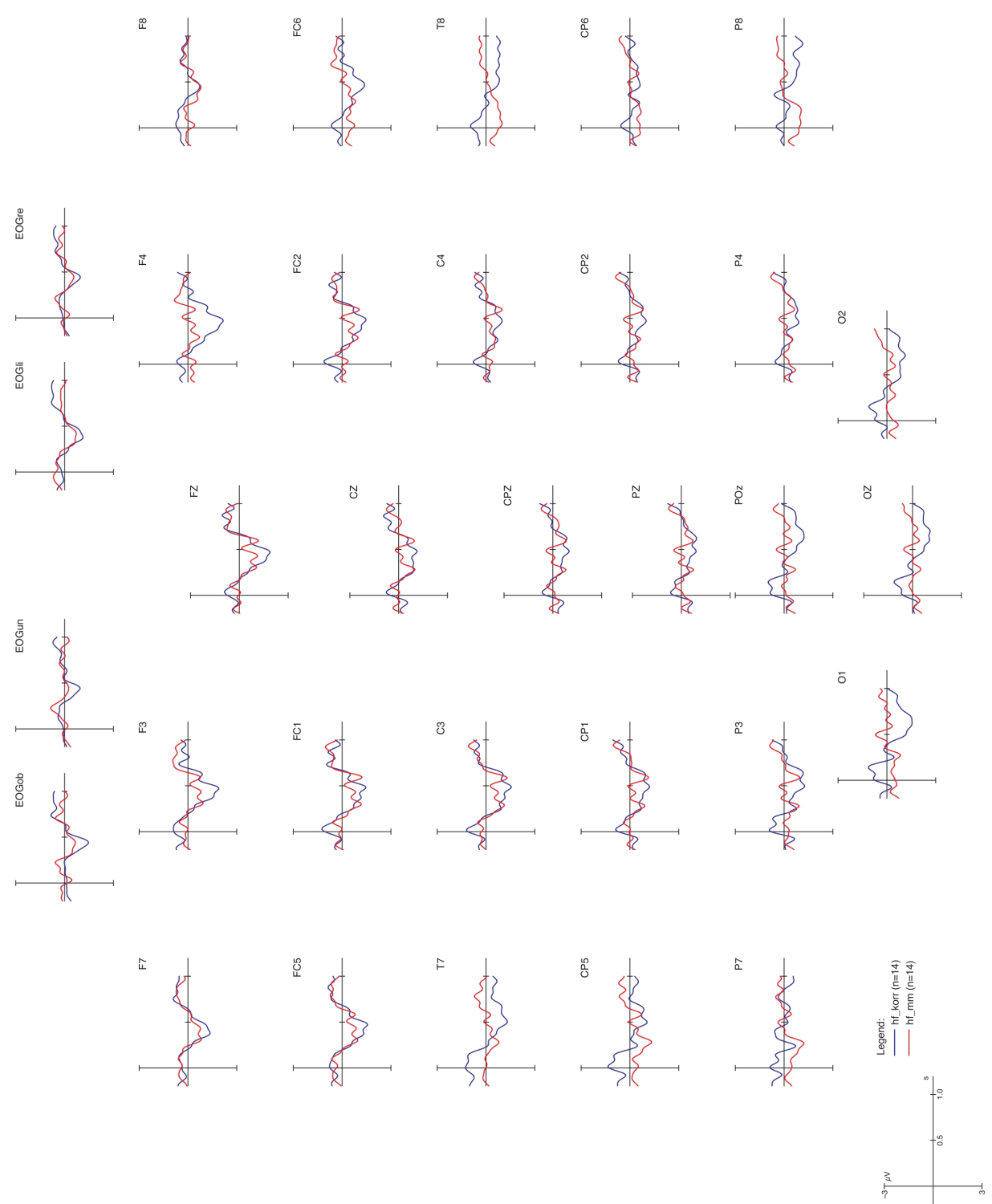


\section{Chapter 3:}

ERPs of agreement violation with plain verbs - trigger sign onset
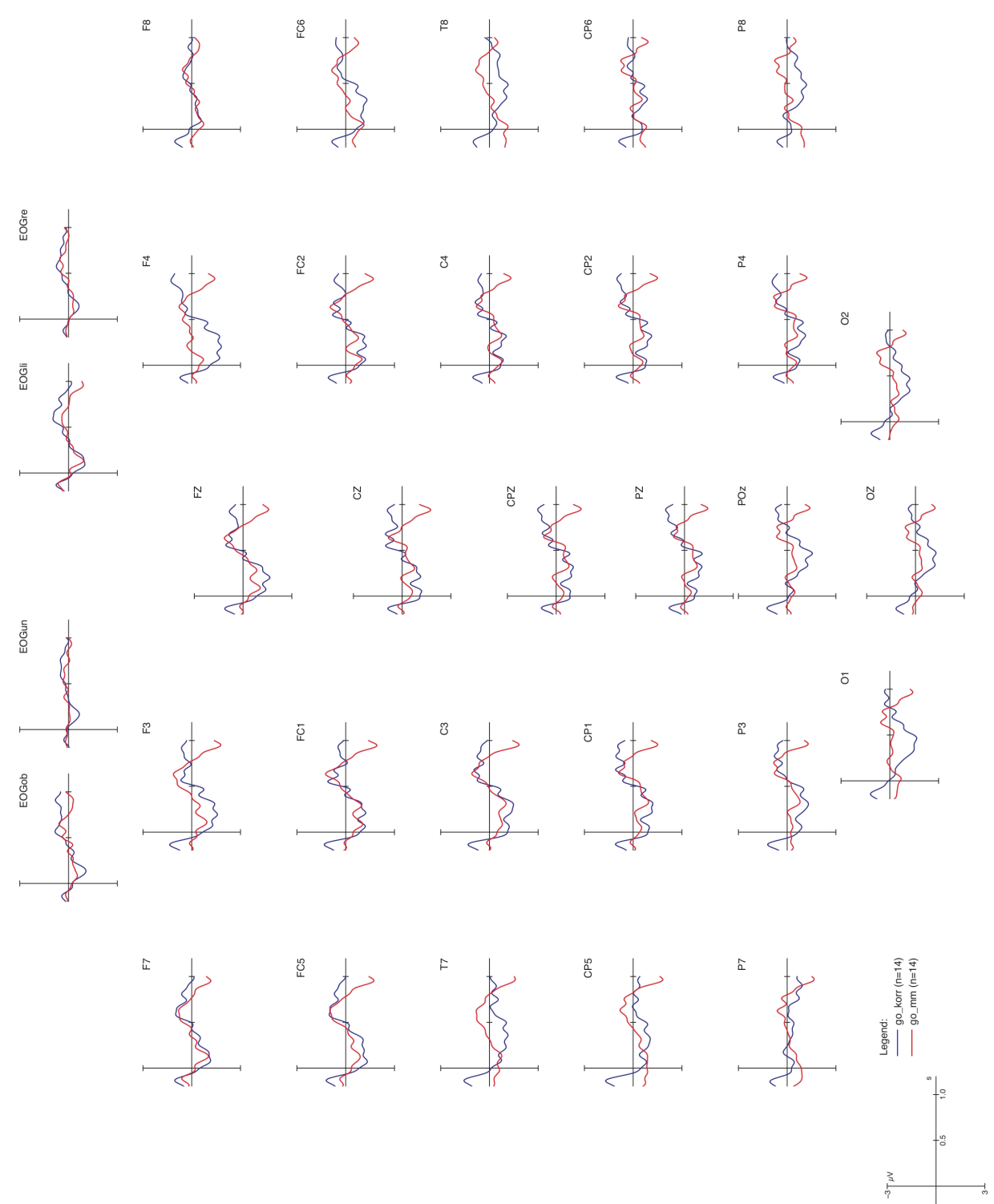


\section{Chapter 3:}

ERPs of agreement violation with plain verbs - trigger eye gaze onset
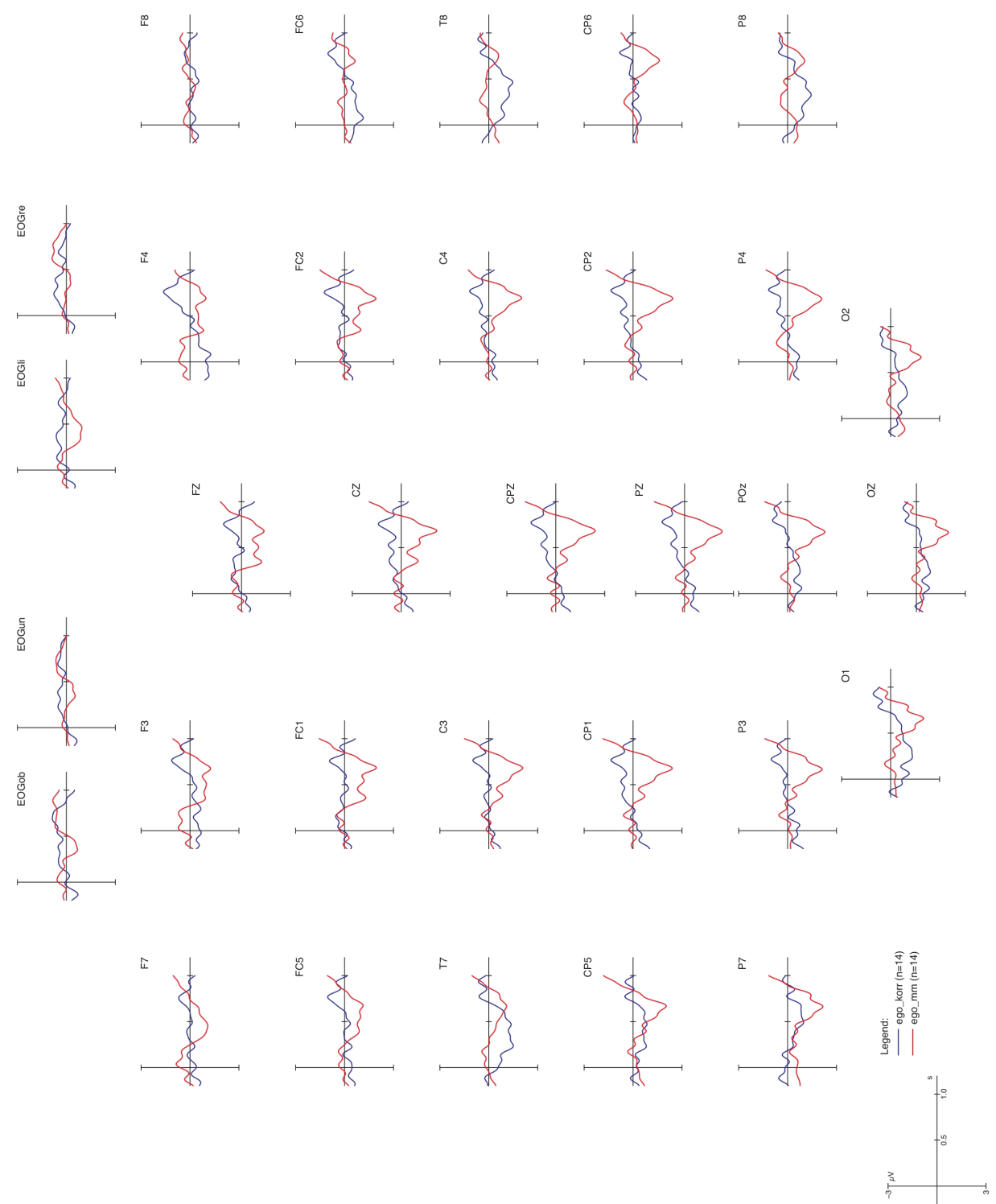


\section{Chapter 3:}

ERPs of agreement violation with plain verbs - trigger mismatch onset
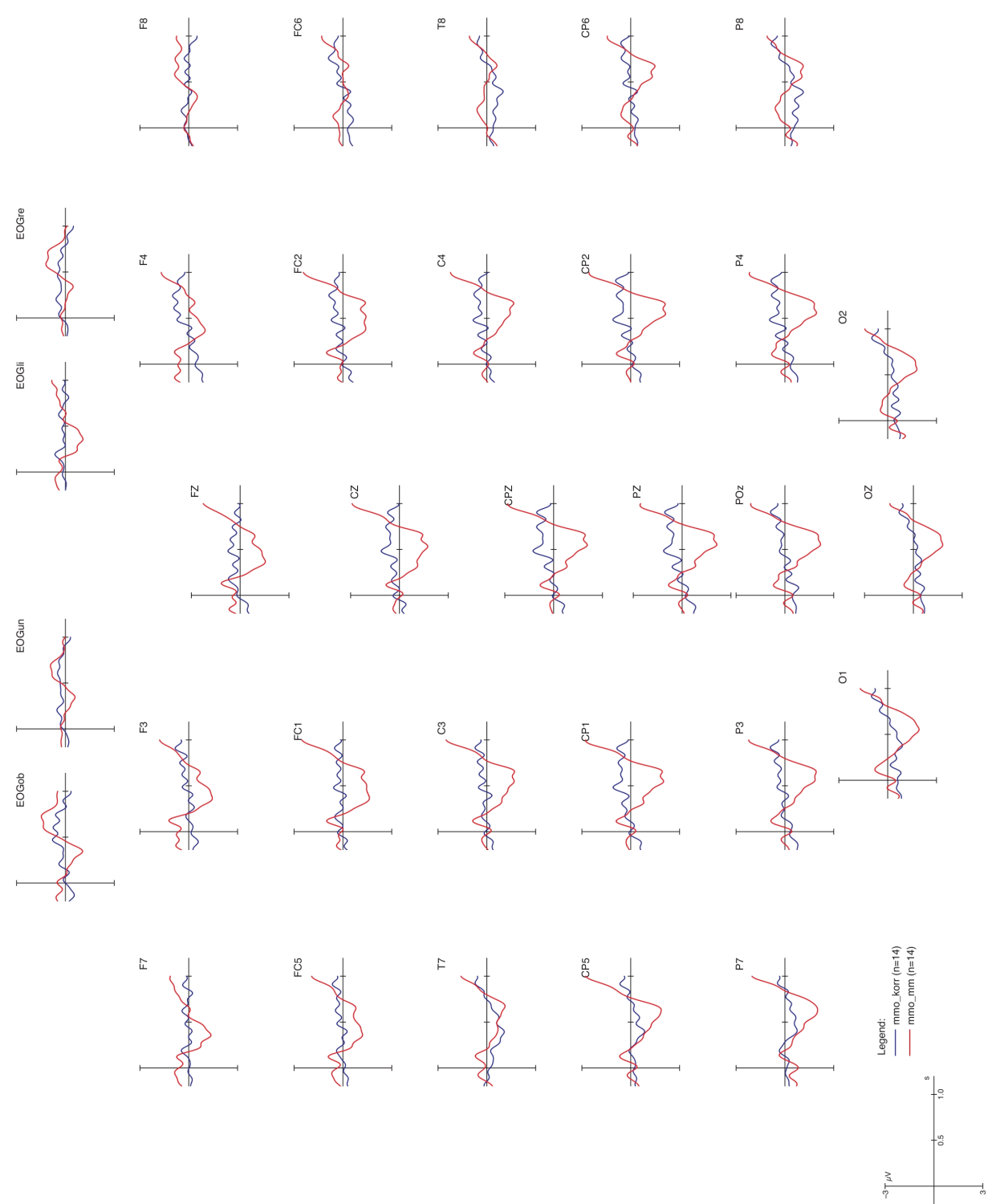


\section{Chapter 3:}

Transkription of feedback to videos with sentences including incorrectly inflected plain verbs.

Video1: I GRANDMA WAIT3a (+body-anchored; +human)

- This generally means „I am waiting for grandma“.

- Something is strange, but that can be ignored.

Video 2: I LAPTOP BUY 3 a (-body-anchored; -human)

- This generally means: „I am buying a laptop.“

- The movement could mean that I pay for it (BEZAHL) (like: I LAPTOP BUY-PAY 3 a).

Video 3: I FLOWER LIKE3a (+body-anchored, -human) Some said, that this is a "ok"-version of LIKE. If you sign fast, LIKE can be this long. The last position can also mean REFUSAL or DISLIKE.

Video 4: I BOY TEST3a (-body-anchored; +human)

This could mean that the boy is small (like: I BOY TEST-SMALL3a).

Or it means that the boy is tested at that place (like: I BOY TEST-THERE 3 a).

Video 5: I PROBLEM IX $\mathrm{X}_{3 \mathrm{a}} \mathrm{KNOW}_{3 \mathrm{a}}$ (+body-anchored; -human) Mostly, people interpreted this as "this is your problem not mine" (like: I PROBLEM IX 3 a KNOW-YOURS3a).

It could also mean "a specific" problem (like: I PROBLEM IX ${ }_{3 a}$ KNOW-THIS ${ }_{3 a}$ ). 


\section{Selbstständigkeitserklärung}

Hiermit versichere ich an Eides statt, dass ich die eingereichte Dissertation

The Processing of German Sign Language sentences. Three event-related potential studies on phonological, morpho-syntactic, and semantic aspects

selbstständig und ohne unerlaubte Hilfe verfasst habe. Anderer als der von mit angegebenen Hilfsmittel und Schriften habe ich mich nicht bedient. Alle wörtlich und sinngemäß den Schriften anderer Autorinnen und Autoren entnommenen Stellen habe ich kenntlich gemacht. Die Abhandlung ist noch nicht veröffentlicht worden und noch nicht Gegenstand eines Promotionsverfahrens gewesen.

Ort, Datum

Jana Hosemann 